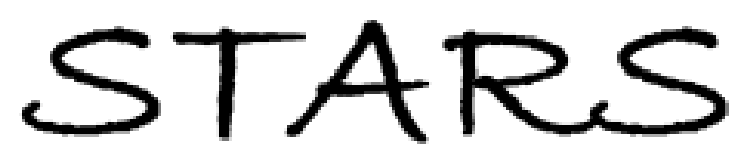

University of Central Florida

STARS

2017

\title{
An Emissive Antenna Correction for The Tropical Rainfall Measuring Mission Microwave Imager (TMI)
}

Faisal Alquaied

University of Central Florida

Part of the Electrical and Computer Engineering Commons

Find similar works at: https://stars.library.ucf.edu/etd

University of Central Florida Libraries http://library.ucf.edu

This Doctoral Dissertation (Open Access) is brought to you for free and open access by STARS. It has been accepted for inclusion in Electronic Theses and Dissertations, 2004-2019 by an authorized administrator of STARS. For more information, please contact STARS@ucf.edu.

\section{STARS Citation}

Alquaied, Faisal, "An Emissive Antenna Correction for The Tropical Rainfall Measuring Mission Microwave Imager (TMI)" (2017). Electronic Theses and Dissertations, 2004-2019. 5533.

https://stars.library.ucf.edu/etd/5533

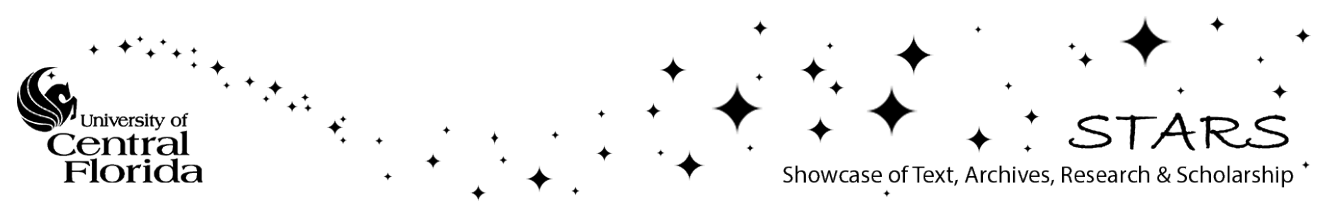




\title{
AN EMISSIVE ANTENNA CORRECTION FOR THE TROPICAL RAINFALL MEASURING MISSION MICROWAVE IMAGER (TMI)
}

by

\section{FAISAL ALQUAIED}

B.S. Electrical Engineering, King Saud University, 2009

M.S. Electrical Engineering, University of Central Florida, 2014

\begin{abstract}
A dissertation submitted in partial fulfillment of the requirements for the degree of Doctor of Philosophy in the Department of Electrical Engineering and Computer Science in the College of Engineering and Computer Science at the University of Central Florida Orlando, Florida
\end{abstract}

\section{Summer Term} 2017

Major Professor: W. Linwood Jones 
(C) 2017 Faisal Alquaied 


\begin{abstract}
This dissertation deals with the radiometric calibration of a satellite microwave radiometer known as the TRMM Microwave Imager (TMI), which operated on NASA's Tropical Rainfall Measuring Mission (TRMM). This multi-frequency, conical-scanning, passive microwave, remote sensor measures the earth's blackbody emissions (brightness temperature, $\mathrm{Tb}$ ) from a low earth orbit and covers the tropics $\left( \pm 35^{\circ}\right.$ latitude). The original scientific objective for TRMM's 3-year mission was to measure the statistics of rainfall in the tropics. However, the mission was quite successful, and TRMM was extended for greater than 17 years to provide a long-term satellite rain measurements, which has contributed significantly to the study of global climate change.

A significant part of the extended TRMM mission was the establishment of a constellation of satellite radiometer that provide frequent global rainfall measurements that enable severe storm warnings for operational hazard forecast by the international weather community. TRMM played a key role by serving as the radiometric calibration standard for the TRMM constellation microwave radiometers.

The objective of this dissertation is to improve the radiometric calibration of TMI and to provide to NASA a new robust, physics-based algorithm for the legacy data processing of the TRMM brightness temperature data product, which will be called TMI 1B11 V8. Moreover, the results of this new procedure have been validated using the double difference techniques with the Global Precipitation Mission Microwave Imager (GMI), which is the replacement satellite mission to TRMM.
\end{abstract}


To my God and my parents, I would be nothing without you.

To my children Abdulaziz and Khalid, seeing both of you is encouraging me to success.

To my brothers, sisters and friends, your support and encouragement is one of the reasons for my achievement. 


\section{ACHNOWLEDGMENTS}

I would like to express my sincere gratitude to my adviser Dr. W. Linwood Jones for his guidance, patience, support, and trust. I promise to pass the knowledge that I got from him to the future science community to improve our world. Also, I would like to thank all of my committee for supporting and commenting my work: Dr. Thomas Wilheit, Dr. Wasfy Mikhael, Dr. Lei Wei, and Dr. Josko Zec.

I would like to thank NASA PPS team: Steven Bilanow for spending a lot of time discussing all of the TRMM geolocation and status, Dr. Racheal Kroodsma for providing the scan bias correction, and Dr Yimin Ji for implementing the code in NASA servers.

I would like to thank the inter-calibration group, which is known by XCAL, for providing me with ideas and comments: Dr. WLinwood Jones, Dr. Thomas Wilheit, Dr. Wesley Berg, and Dr. Darren McKague. Also, I would like to thank Dr. David Draper from Ball Aerospace Company, who reviewed and validated my work in the Antenna Pattern Correction (APC).

I would like to thank my colleges in CFRSL for being my second family. Also, I would like to thank Dr. Spenser Farrar for providing me with the STK code and Dr. Zoubair Gahzi for spending a lot of time sharing the concept of radiometers.

I would like to acknowledge my organization King Abdulaziz City for Science and Technology (KACST) for sponsoring my PhD degree. Also, I would like to acknowledge NASA for funding tools that speed up my analysis. 


\section{TABLE OF CONTENT}

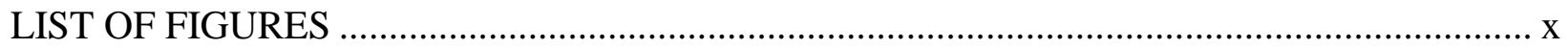

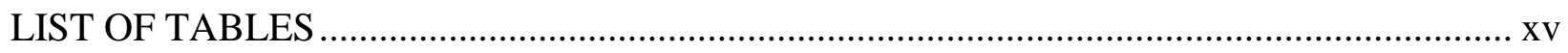

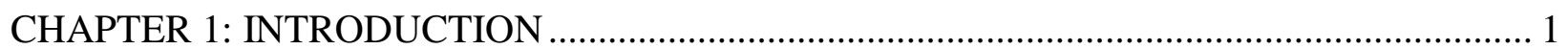

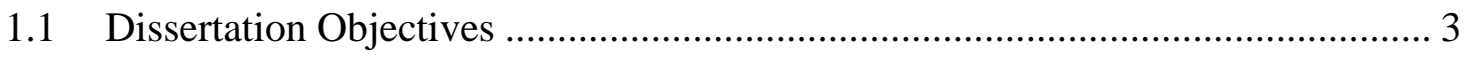

1.2 Dissertation Overview ........................................................................... 4

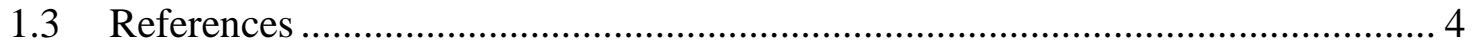

CHAPTER 2: TRMM MICROWAVE IMAGER ............................................................ 6

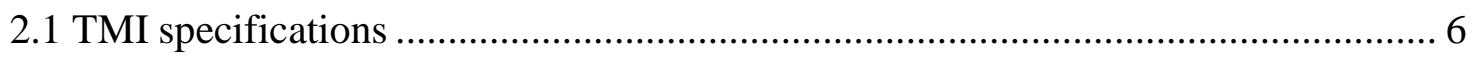

2.2 Radiometric Calibration ................................................................................. 10

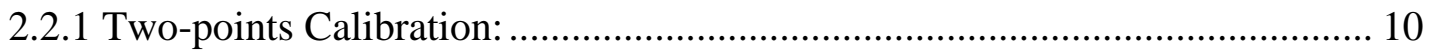

2.2.2 Along Scan Calibration Error: ................................................................ 13

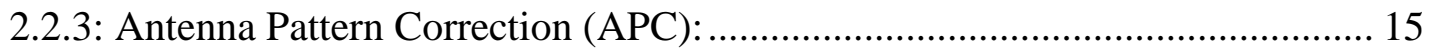

2.3 TMI 1B11 Tb Product.................................................................................... 20

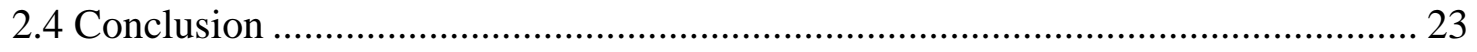

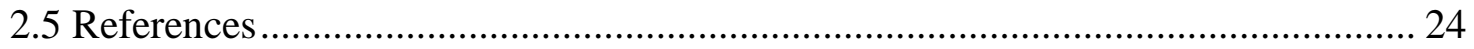

CHAPTER 3: HOT LOAD CORRECTION ....................................................................... 27

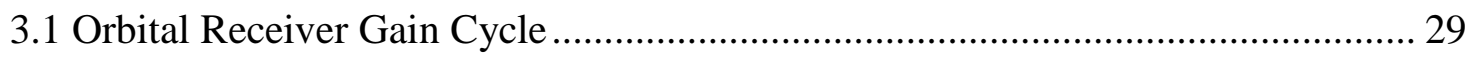

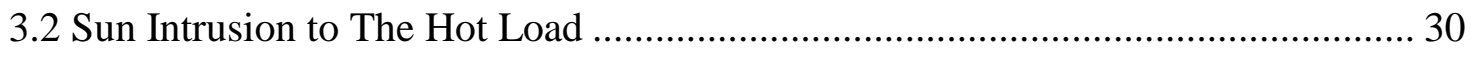




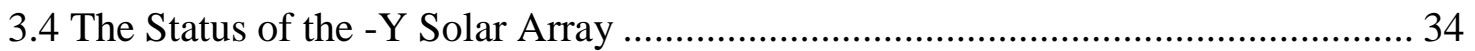

3.5 Approach to Correct the Hot Load Brightness Temperature ............................... 36

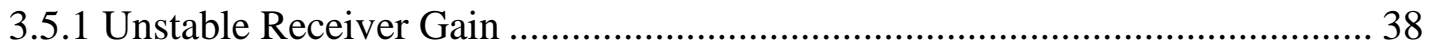

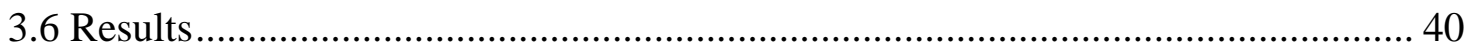

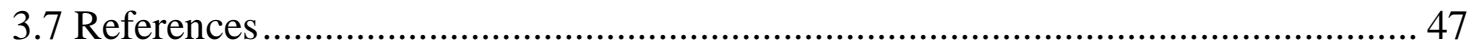

CHAPTER 4: EMISSIVE ANTENNA CORRECTION ................................................... 48

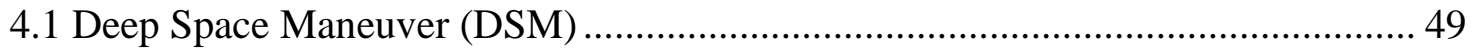

4.2 Emissivity Coefficients ......................................................................... 56

4.3 Physical Temperature During Normal Operation .......................................... 60

4.3.1 Main Reflector Physical Temperature (Pre-boost Altitude $=350 \mathrm{~km}) \ldots \ldots \ldots \ldots 61$

4.3.2 Main Reflector Physical Temperature (Post-boost Altitude $=405$ km) ......... 64

4.3.3 Main Reflector Physical Temperature (end of mission) ................................6 67

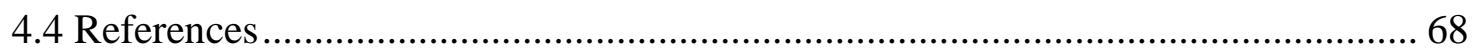

CHAPTER 5: 1B11 V8 VALIDATION ..................................................................... 71

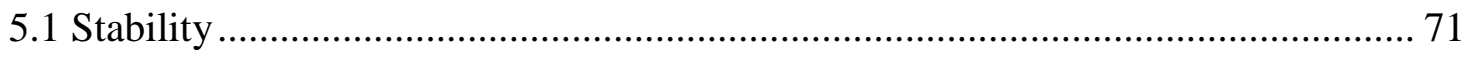

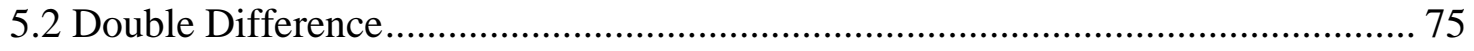

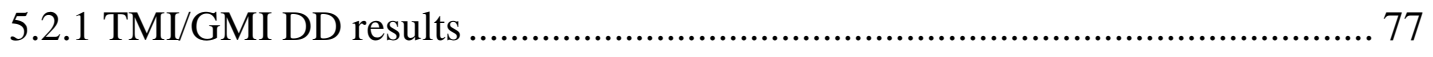

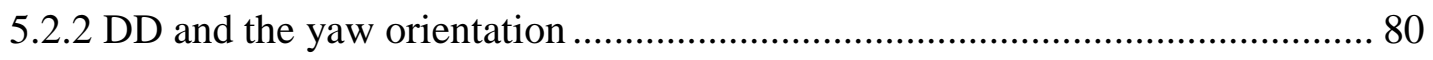




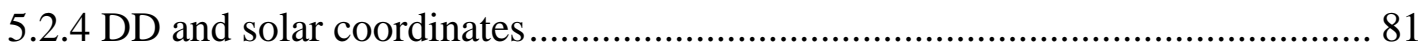

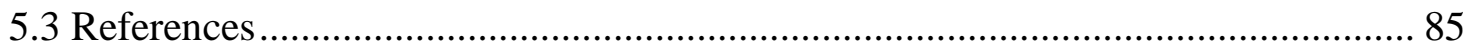

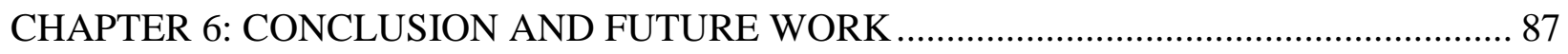

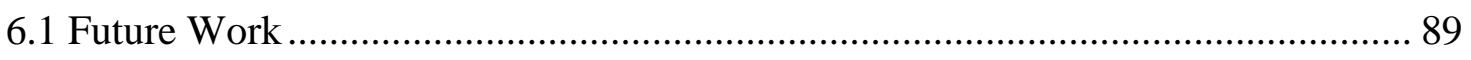

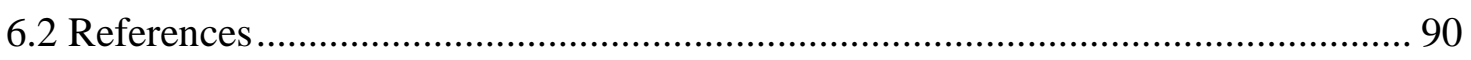

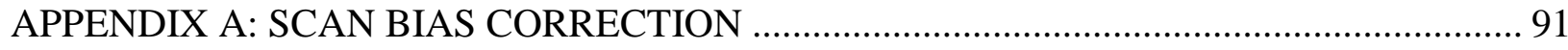

APPENDIX B: DERVATION OF ANTENNA PATTERN CORRECTION COEFFICIENTS . 97

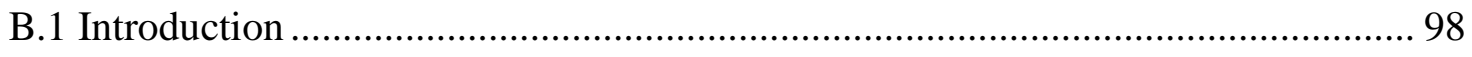

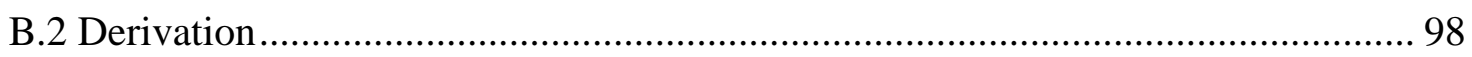

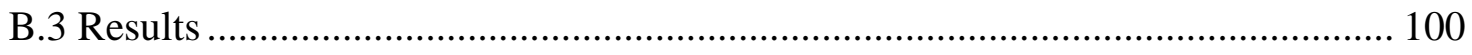

APPENDIX C: THE STATUS OF -Y SOLAR ARRAY ……………………….................... 102

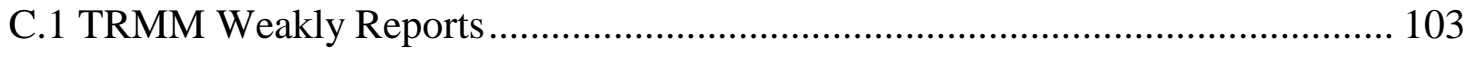

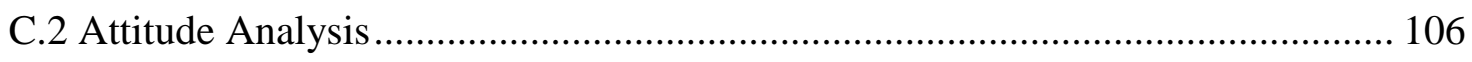

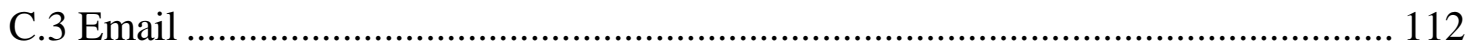

APPENDIX D: HOT LOAD CORRECTION RESULTS FOR ALL CHANNELS AND ALL

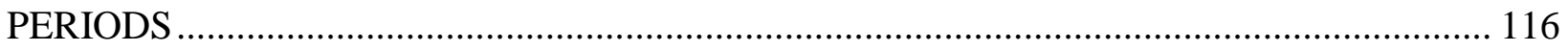

APPENDIX E: PHYSICAL TEMPERATURE OF THE MAIN REFLECTOR AT YAW 180125 APPENDIX F: DOUBLE DIFFERENCE (DD) RESULTS FOR VERSIONS 7 AND 8 ......... 128 


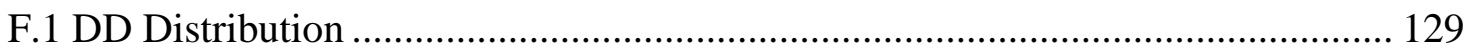

F.2 DD Based on Solar Coordinates ........................................................... 131 


\section{LIST OF FIGURES}

Figure 1.1: TRMM 3B42 global rainfall 7-day image (Source: [8])...................................... 3

Figure 2.1: Schematic view of the scan geometries for TMI, PR, and VIRS (Source: [1])......... 7

Figure 2.2: Mechanical configuration of the TMI instrument. (source: [2])............................ 9

Figure 2.3: TMI's feed horns sampling based on the azimuth rotation angle. (source: [2])........ 10

Figure 2.4: Block diagram of the two-point calibration for a TMI..................................... 11

Figure 2.5: The radiometer transfer function from the two-point calibration........................... 12

Figure 2.6: Example of Scan bias correction for channel (10.65 GHz, V. pol) for Ocean and land

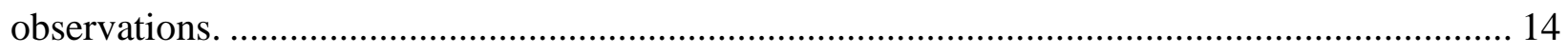

Figure 2.7: Definition of spill-over beam pattern relative to the feed horn primary pattern and the

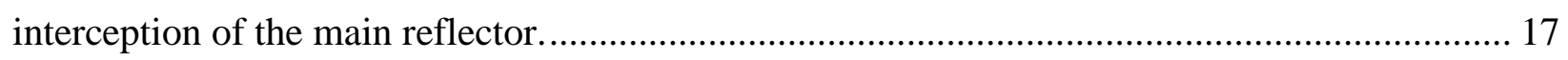

Figure 2.8: The ratio between the horizontal and vertical polarization of the modeled $\mathrm{Tb}$ for

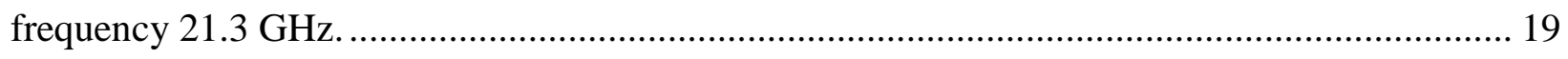

Figure 2.9: Flow chart of the TMI 1B11 v6 procedure ........................................................ 22

Figure 2.10: Flow chart of the TMI 1B11 v7 procedure.................................................... 22

Figure 2.11: Flow chart of the TMI 1B11 v8 procedure..................................................... 23

Figure 3.1: TMI parameters for $10.65 \mathrm{~V}$ channel during TRMM orbit \# 87736: (a) Hot counts,

(b) measured hot load physical temperature, (c) cold counts, (d) inverse-xfer gain and (e)

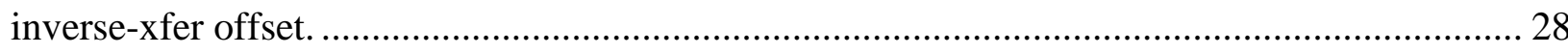

Figure 3.2: Possible hot load exposure during the ground test of the TMI. (Source: [3])........... 30

Figure 3.3: Sun intrusion heating the pyramidal tips of the hot load..................................... 32

Figure 3.4: Solar coordinates relative to TRMM (TRMM model source: [4])......................... 34 
Figure 3.5: Polynomial fit for the radiometer gain time series to produce the hot load correction.

Figure 3.6: Receiver gain of channel $21.3 \mathrm{~V}$ and $85.5 \mathrm{~V}$ at solar beta angle $=51.3^{\circ} \ldots \ldots \ldots \ldots \ldots . . \ldots$

Figure 3.7: Receiver gain of channel $21.3 \mathrm{~V}$ and $85.5 \mathrm{~V}$ at solar beta angle $=5.5^{\circ} \ldots \ldots \ldots \ldots \ldots \ldots . . \ldots$

Figure 3.8: Hot load correction table for channel $10.65 \mathrm{~V}$, yaw0, and period-1...................... 42

Figure 3.9: The effect of the intersection between the cold sky reflector beam and the -Y solar

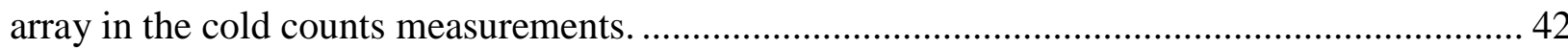

Figure 3.10: The intersection between the cold sky reflector beam and the -Y solar array at high

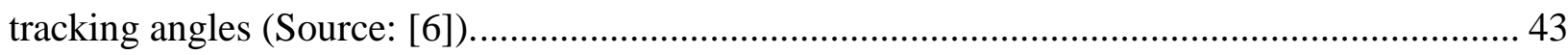

Figure 3.11: Hot load correction table for channel $10.65 \mathrm{~V}$, yaw0, and period 2.................... 45

Figure 3.12: Hot load correction table for channel $10.65 \mathrm{~V}$, yaw0, and period 3.................... 45

Figure 3.13: Hot load correction table for channel $10.65 \mathrm{~V}$, yaw0, and period 4. ................... 46

Figure 3.14: The hot load correction effect on the antenna temperature. ................................ 46

Figure 4.1: Example of a typical DSM showing TMI phases for the spacecraft yaw $=0$ degrees.

Figure 4.2: Measured antenna temperature (10.65V) shown as a colored trace along the satellite

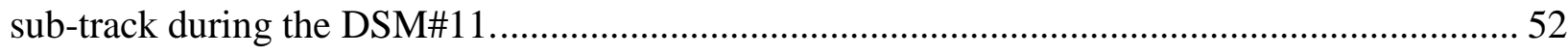

Figure 4.3: TRMM phases during the DSM at yaw 90 degrees. ......................................... 53

Figure 4.4: Antenna brightness temperature (10.65 V) during 4 phases of the DSM\#18, performed at a spacecraft yaw of $90^{\circ}$.

Figure 4.5: Measured antenna temperature (10.65V) during the DSM\#18 plotted as a colored trace on the TRMM ground track. 
Figure 4.6: Derived main reflector emissivity coefficients for four yaw-90 DSM's.

Figure 4.7: Flow chart of the procedure used to estimate TMI MR physical temperature during normal operation. 61

Figure 4.8: 2D matrix of MR physical temperature (yaw 0 and pre-boost altitude $=350 \mathrm{~km}$ )... 62 Figure 4.9: Tphy relative to time since eclipse for yaw 0 and pre-boost at solar beta angle $=43^{\circ}$.

Figure 4.10: 2D matrix of MR physical temperature (yaw 0 and post-boost altitude $=405 \mathrm{~km}$ ). 65 Figure 4.11: Comparing 1D Tphy between pre-boost $\&$ post-boost at solar beta angle $=43^{\circ} \ldots . .66$ Figure 4.12: Comparison of the post-boost 1D Tphy for TMI and of the measured GMI Tphy at solar beta angle $=43^{\circ}$ 66

Figure 4.13:TRMM altitude during the last year of mission. 68

Figure 5.1: The procedure to calculate the Single differences between observed and modeled brightness temperature. 74

Figure 5.2: The single differences quarterly results for both orientations (yaw 0 and 180 degrees) for 15 years (channel $10.65 \mathrm{~V})$. 75

Figure 5.3: DD distribution of channel $10.65 \mathrm{~V}$ for versions 6,7 , and 8 . 79

Figure 5.4: Mean of DD of each channel and each yaw orientation for both version 7 and 8 .... 80 Figure 5.5: DD depends on earth's latitude for versions 7 (blue) and 8 (red). 83

Figure 5.6: DD anomaly between version 7 and 8 based on the solar coordinates of channel 19.35 V. 84

Figure 5.7: Correlation of 19.35 V DD bias with the GMI earth scene brightness temperature for versions 7 (red) and 8 (blue). 84 
Figure 6.1: Scan bias correction for channel $10.65 \mathrm{~V}$ for $\mathrm{Ta}=170 \mathrm{~K}$ and $\mathrm{Ta}=280 \mathrm{~K} . \ldots \ldots \ldots \ldots \ldots . . . . . .92$

Figure 6.2: Scan bias correction for channel $10.65 \mathrm{H}$ for $\mathrm{Ta}=90 \mathrm{~K}$ and $\mathrm{Ta}=280 \mathrm{~K}$................. 93

Figure 6.3: Scan bias correction for channel $19.35 \mathrm{~V}$ for $\mathrm{Ta}=200 \mathrm{~K}$ and $\mathrm{Ta}=280 \mathrm{~K} . \ldots \ldots \ldots \ldots \ldots . . . . . . .93$

Figure 6.4: Scan bias correction for channel $19.35 \mathrm{H}$ for $\mathrm{Ta}=140 \mathrm{~K}$ and $\mathrm{Ta}=280 \mathrm{~K}$............... 94

Figure 6.5: Scan bias correction for channel 21.3 V for $\mathrm{Ta}=220 \mathrm{~K}$ and $\mathrm{Ta}=280 \mathrm{~K}$................. 94

Figure 6.6: Scan bias correction for channel $37.00 \mathrm{~V}$ for $\mathrm{Ta}=215 \mathrm{~K}$ and $\mathrm{Ta}=280 \mathrm{~K}$............... 95

Figure 6.7: Scan bias correction for channel $37.00 \mathrm{H}$ for $\mathrm{Ta}=160 \mathrm{~K}$ and $\mathrm{Ta}=280 \mathrm{~K} . \ldots \ldots \ldots \ldots \ldots . . . . .95$

Figure 6.8: Scan bias correction for channel $85.50 \mathrm{~V}$ for $\mathrm{Ta}=260 \mathrm{~K}$ and $\mathrm{Ta}=280 \mathrm{~K} . \ldots \ldots \ldots \ldots \ldots . . . . . . .96$

Figure 6.9: Scan bias correction for channel $85.50 \mathrm{H}$ for $\mathrm{Ta}=230 \mathrm{~K}$ and $\mathrm{Ta}=280 \mathrm{~K} . \ldots \ldots \ldots \ldots . . . . .96$

Figure 6.10: Attitude analysis shows the non-feathered solar array of orbit $25976 \ldots \ldots \ldots \ldots \ldots . . . . .108$

Figure 6.11: Attitude analysis shows the feathered solar array of orbit $25977 \ldots \ldots \ldots \ldots \ldots \ldots \ldots \ldots . . . . . . . . .109$

Figure 6.12: Attitude analysis shows the feathered solar array of orbit 25978..................... 110

Figure 6.13: Attitude analysis shows the non-feathered solar array of orbit $25979 \ldots \ldots \ldots \ldots \ldots \ldots . . . . .111$

Figure 6.14: Hot load correction for all channels (yaw0, period 1) ...................................... 117

Figure 6.15: Hot load correction for all channels (yaw0, period 2).................................... 118

Figure 6.16: Hot load correction for all channels (yaw0, period 3)...................................... 119

Figure 6.17: Hot load correction for all channels (yaw0, period 4).................................... 120

Figure 6.18: Hot load correction for all channels (yaw180, period 1)................................. 121

Figure 6.19: Hot load correction for all channels (yaw180, period 2) .................................. 122

Figure 6.20: Hot load correction for all channels (yaw180, period 3) .................................. 123

Figure 6.21: Hot load correction for all channels (yaw180, period 4).................................... 124

Figure 6.22: 2D matrix of MR physical temperature (yaw 180 and pre-boost)..................... 126 
Figure 6.23: 2D matrix of MR physical temperature (yaw 180 and post-boost).................... 127

Figure 6.24: Double Difference (DD) distribution for V7 and V8 (all channels, yaw 0).......... 129

Figure 6.25: Double Difference (DD) distribution for V7 and V8 (all channels, yaw 180)...... 130

Figure 6.26: 2D Double Difference (DD) differences between V7 and V8 based on solar

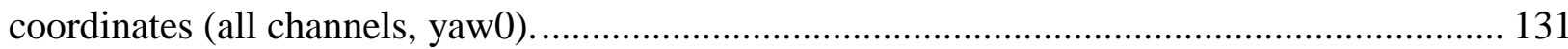

Figure 6.27: 2D Double Difference (DD) differences between V7 and V8 based on solar

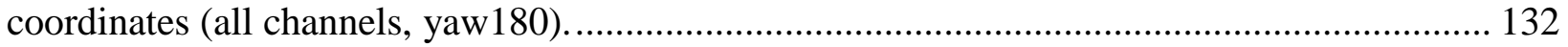

Figure 6.28: Scatter plot with linear regression for the high DD anomaly for V7 and V8 (all

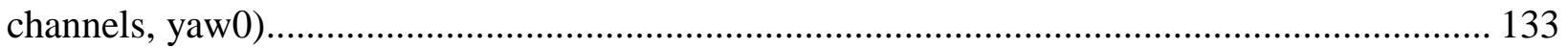

Figure 6.29: Scatter plot with linear regression for the high DD anomaly for V7 and V8 (all

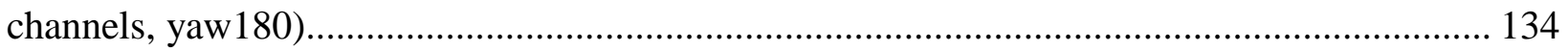




\section{LIST OF TABLES}

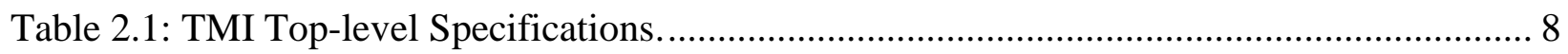

Table 2.2: The pre-launch cross polarization and spill-over coefficients.................................... 15

Table 2.3: TMI's antenna pattern correction (APC) coefficients ................................................. 19

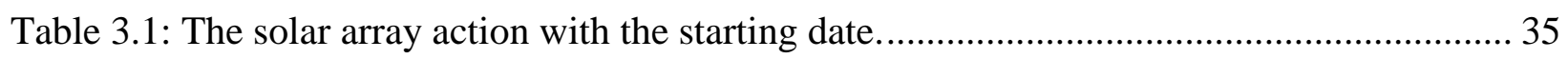

Table 3.2: Orbits range for each orientation and period............................................................. 36

Table 4.1: Deep Space Maneuvers (DSMs) during TRMM mission............................................. 50

Table 4.2: Scan number, scan positions, and estimated Tphy for each yaw-90 DSM................... 58

Table 4.3: Main reflector emissivity coefficients for 1B11 V8 and 1B11 V7 ..............................59

Table 5.1: Center frequency and bandwidth of TMI and GMI channels..................................... 76

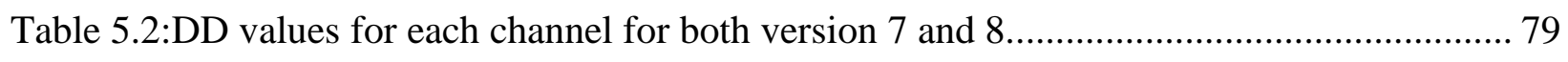

Table 6.1: TMI's APC coefficients for version 1B11 V5 and earlier........................................... 100

Table 6.2: TMI's APC coefficients for version 1B11 V6\&7....................................................... 101

Table 6.3: TMI's APC coefficients for version 1B11 V8......................................................... 101 


\section{CHAPTER 1: INTRODUCTION}

The Tropical Rainfall Measuring Mission (TRMM) satellite was launched on November 27, 1997 as a joint effort between the National Aeronautics and Space Administration (NASA) and the Japan Aerospace Exploration Agency (JAXA). The main objective of the mission was to study the statistics of rainfall in the tropics, specifically; to determine the average rain accumulation over a $5^{\circ} \times 5^{\circ}$ (latitude/longitude) region on a monthly basis during a prime mission of 3 years [1]. Later, during the extended satellite mission, the science objectives were expanded to include global measurements by the formation of a constellation of cooperative weather satellites with microwave radiometers to provide observational data for studying atmospheric circulation by measuring the tropical precipitation, water vapor, and clouds. The investigation of the interaction between these weather's parameters is extremely important since it leads to understand our climate, through the study of the Earth's hydrological cycle [2]

To achieve the goal of this mission, TRMM carried four main instruments: TRMM Microwave Imager (TMI), the Precipitation Radar (PR), the Visible and Infrared Scanner (VIRS) and the Lightning Imaging Sensor (LIS). TRMM flew in a non-sun synchronous orbit with an inclination of 35 degrees at $350 \mathrm{Km}$ altitude, with an orbit period of $\sim 90$ minutes. Since the orbit was non-sun synchronous, about 1/3 of this revolution time was in eclipse; and the orbit precession was $\sim 1 / 2$ hour in local time each day [3]. TRMM prime mission was planned to be 3 years; however, with the purpose of extending the science mission [4], the satellite orbit altitude was increased to $405 \mathrm{Km}$ in Aug, 2001. Moreover, multiple satellites carrying microwave radiometers were added to form a constellation for global rain measurements. After successfully performing for $>17$ years, 
TRMM was deactivated on April 8, 2015, when the fuel was depleted and the orbit could no longer be maintained, and it re-entered the Earth's atmosphere on June 15, 2015.

Since TRMM and its constellation of cooperative satellites have provided almost two decades of brightness temperature observations, these observations have the potential to be used for climate studies. However, this requires a very strict inter-satellite radiometric calibration between sensors to assure consistent environmental parameter retrievals. Thus, the inter-satellite radiometric calibration working group (XCAL) was formed and charged with the responsibility of developing robust techniques to preform and maintain the radiometric calibration of constellation radiometer instruments, relative to one another. Because the TRMM orbit was non-sun synchronous, it had frequent near-simultaneous collocations with the other radiometers in polar orbits, therefore it is the best choice to be the radiometric transfer standard for the constellation. Thus, by having a standard calibration for all these instruments, the global brightness temperature dataset was unified [5]. After that, the constellation brightness temperatures were used to retrieved precipitation and other important global environment parameters. For example, the TRMM 3B42 7-day global rainfall product is shown in Figure 1.1.

However, for TMI to serve as the radiometric transfer standard, it was necessary to carefully assess the calibration accuracy and stability of this instrument, and this is the goal of this dissertation. Previous to this research, there were several efforts to develop an algorithm to process TMI sensor data to yield the desired earth scene brightness temperature that resulted in several versions of the TMI brightness temperature products 1B11 [6-7], which are described in the chapters that follow. 
Therefore, in this dissertation, the development of the final TMI 1B11 (V8) brightness temperature product will be described in detail. Moreover, with this final data processing algorithm, all known instrument radiometric calibration anomalies are corrected based upon rigorous electromagnetic principles and/or on-orbit radiometric measurements.

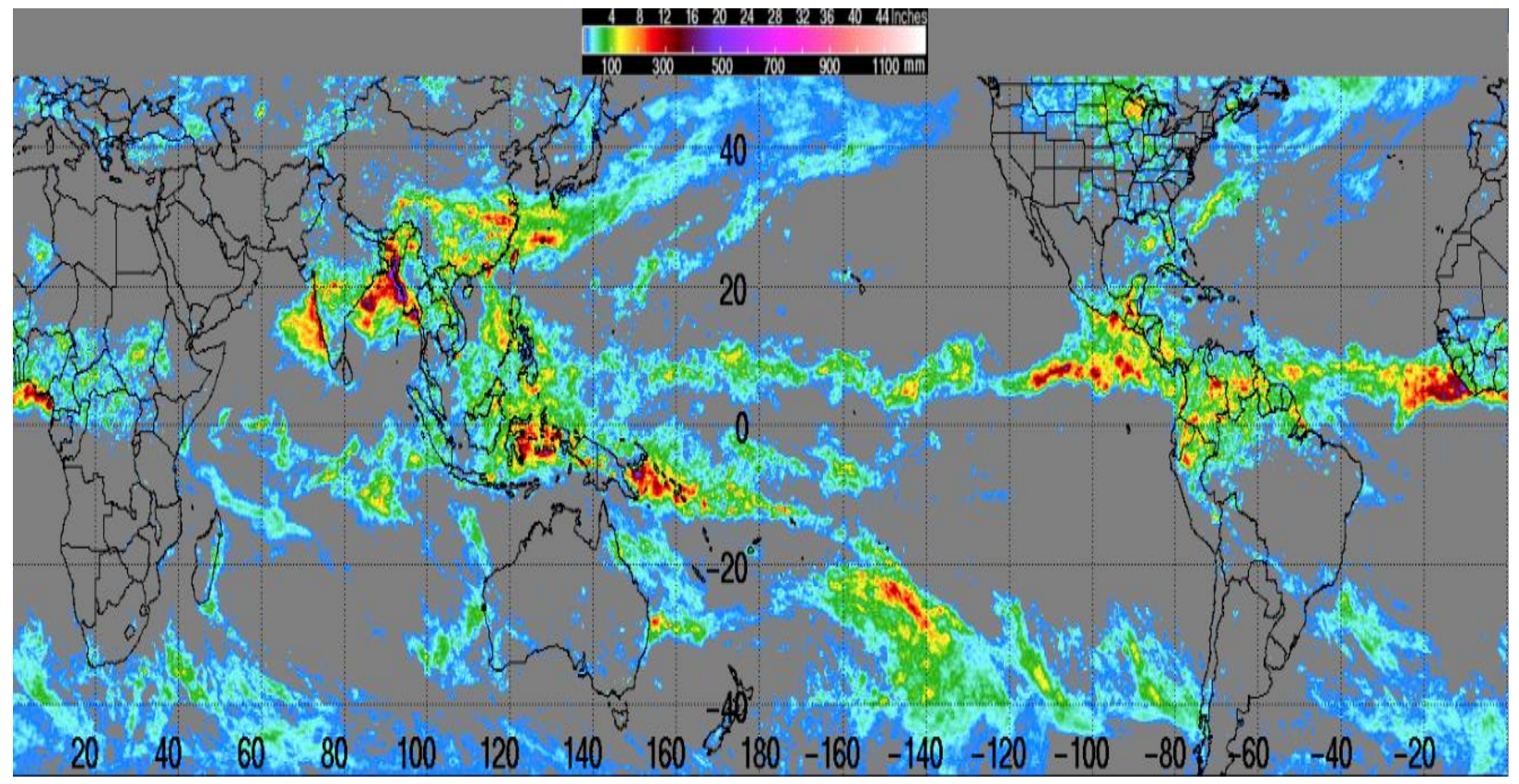

Figure 1.1: TRMM 3B42 global rainfall 7-day image (Source: [8]).

\subsection{Dissertation Objectives}

- To derive a robust emissive reflector antenna correction for the TMI counts to brightness temperature algorithm 1B11 Version-8.

- To analyze deep space calibration tests, to derive the main reflector emissivity coefficient for each TMI channel.

- To use the TMI XCAL radiative transfer model single differences to derive the physical temperature of the main reflector for the entire 17 year TMI dataset. 
○ To provide an independent hot-load correction for errors introduced by solar intrusion.

\subsection{Dissertation Overview}

This dissertation is organized to cover the TMI 1B11 version 8 algorithm development in detail. Chapter 2, will describe the TRMM Microwave Imager (TMI) radiometric calibration procedure and observed on-orbit calibration issues. Subsequently, Chapter 3 will explain the hot load anomalies caused by the sun intrusion and present an effective mitigation solution. In Chapter 4, the emissive antenna anomaly and its solution (correction algorithm) will be described. The results of on-orbit validation are given in Chapter 5. Finally, the conclusions will be presented in Chapter 6.

\section{$1.3 \underline{\text { References }}$}

[1] J. Simpson, R. F. Adler, and G. R. North, "A proposed tropical rainfall measuring mission (TRMM) satellite," Bulletin of the American Meteorological Society, vol. 69, no. 3, pp. 278-295, 1988.

[2] Science Plan for NASA's Science Mission Directorate 2007-2016, National Aeronautics and Space Administration (NASA), Chapter 4, 2007, available at: https://science.nasa.gov/about-us/science-strategy.

[3] C. Kummerow, W. Barnes, T. Kozu, J. Shiue, and J. Simpson, "The tropical rainfall measuring mission (TRMM) sensor package," Journal of atmospheric and oceanic technology, vol. 15, no. 3, pp. 809-817, 1998. 
[4] N. R. Council, Assessment of the Benefits of Extending the Tropical Rainfall Measuring Mission: A Perspective from the Research and Operations Communities, Interim Report. National Academies Press, 2006.

[5] T. T. Wilheit, "Comparing calibrations of similar conically scanning window-channel microwave radiometers," IEEE Transactions on Geoscience and Remote Sensing, vol. 51, no. 3, pp. 1453-1464, 2013.

[6] F. J. Wentz, P. Ashcroft, and C. Gentemann, "Post-launch calibration of the TRMM microwave imager," IEEE Transactions on Geoscience and Remote Sensing, vol. 39, no. 2, pp. 415-422, 2001.

[7] S. K. Biswas, K. Gopalan, W. L. Jones, and S. Bilanow, "Correction of time-varying radiometric errors in TRMM microwave imager calibrated brightness temperature products," IEEE Geoscience and Remote Sensing Letters, vol. 7, no. 4, pp. 851-855, 2010.

[8] National Aeronautics and Space Administration (NASA), Goddard space flight center, TRMM (2017), available at: https://pmm.nasa.gov/TRMM/realtime-3hr-7day-rainfall 


\section{CHAPTER 2: TRMM MICROWAVE IMAGER}

In this Chapter, the TRMM Microwave Imager (TMI) will be discussed, including TMI specifications and details of the radiometric calibration. TMI was built upon the heritage of the Special Sensor Microwave Imager (SSM/I), which was first launched on the U.S. Defense Meteorological Satellite Program (DMSP) in 1987. Both sensors have the same specification for 6 channels, but TMI has an extra feed horn that carries two $10 \mathrm{GHz}$ channels; also, the water vapor channel was adjusted in the TMI to be $21.3 \mathrm{GHz}$ (instead of $22.235 \mathrm{GHz}$ in the SSM/I) to improve the measurement sensitivity. Although both have a similar main reflector antenna, the TMI has higher resolution than the SSM/I due to the altitude differences (350-405 km compared with 800 $\mathrm{km})[1]$.

\section{$\underline{2.1 \text { TMI specifications }}$}

TMI was a total power radiometer with a conical scanning antenna mechanism that spun 31.6 revolutions/minute (RPM), completing a full rotation in 1.9 seconds. The zero azimuth position looked in the $+\mathrm{X}$ direction of TRMM, as illustrated in Figure 2.1. It obtained brightness temperature measurements along a circular path on the surface for a $759 \mathrm{~km}$ swath while TRMM flew at $350 \mathrm{~km}$ altitude. TRMM was required to avoid the sun Line of Sight (LOS) on the + Y side, where the Visible and Infrared Radiometer (VIRS) cooler and the TMI's cold sky reflector were placed to view cold space. To achieve this requirement, TRMM performed a yaw flip (yaw 0 and yaw 180 degree) every 2 to 4 weeks [1], when the sun LOS passed through the orbital plane. 


\section{X, Y, Z: Spacecraft coordinate system}

(right handed)

* Nominally coincident with spacecraft flight direction with velocity, $\underline{\mathbf{v}}: 7.3 \mathrm{~km} / \mathrm{sec}$

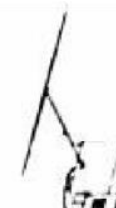

$+\mathrm{Y}:$ VIRS cooler side

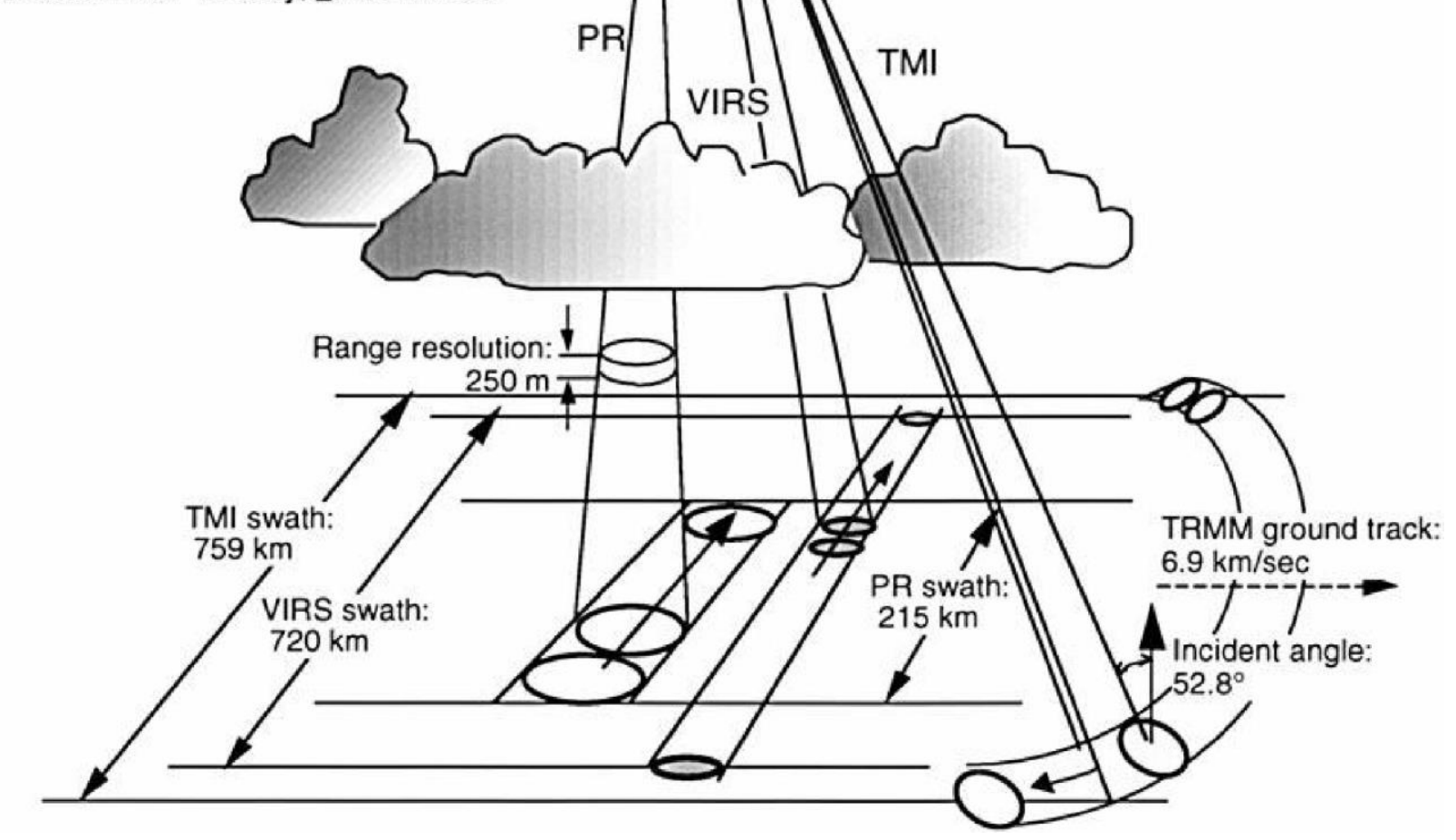

Figure 2.1: Schematic view of the scan geometries for TMI, PR, and VIRS (Source: [1]).

The main components and the operation of TMI are described in Figs. 2.2 and 2.3. TMI was comprised of nine channels with five different frequencies, namely; 10.65, 19.35, 21.30, and 85.5 GHz. All frequencies were dual polarized, vertical (V) and horizontal (H) except for 21.3 $\mathrm{GHz}$ that was V-pol only. Also, there were two feed horns: one for the $10.65 \mathrm{GHz}$ (both polarizations) and another for the remaining channels, where the multi-frequency feed was a heritage design from SSMI. Further, each channel had a dedicated receiver that was placed in the 
rotating canister of TMI. The detailed specification for each receiver with the Instant Field of View (IFOV) is described in Table $2.1[1,2]$.

Table 2.1: TMI Top-level Specifications.

\begin{tabular}{|l|c|c|c|c|c|}
\hline Center Frequency (GHz) & $\mathbf{1 0 . 6 5}$ & $\mathbf{1 9 . 3 5}$ & $\mathbf{2 1 . 3 0}$ & $\mathbf{3 7 . 0 0}$ & $\mathbf{8 5 . 5 0}$ \\
\hline Polarization & $\mathrm{V} / \mathrm{H}$ & $\mathrm{V} / \mathrm{H}$ & $\mathrm{V}$ & $\mathrm{V} / \mathrm{H}$ & $\mathrm{V} / \mathrm{H}$ \\
\hline Bandwidth (MHz) & 100 & 500 & 200 & 2000 & 3000 \\
\hline Tb sensitivity NE $\mathbf{T}$ (K) & $0.63 / 0.54$ & $0.50 / 0.47$ & 0.71 & $0.36 / 0.31$ & $0.52 / 0.93$ \\
\hline IFOV (km x km) @ 350 Km & $63 \times 37$ & $30 \times 18$ & $23 \times 18$ & $16 \times 9$ & $7 \times 5$ \\
\hline IFOV (km x km) @ 405 Km & $73 \times 43$ & $35 \times 21$ & $26 \times 21$ & $18 \times 10$ & $8 \times 6$ \\
\hline
\end{tabular}

The two feed horns viewed the main reflector, which was a parabolic mirror with a diameter equal to 0.67 meters, that received the Earth brightness temperature at a cone angle of 49 degrees. Both feeds and reflector rotated to measure $\mathrm{Tb}$, symmetrically about the satellite ground track, over an azimuth range of \pm 65 degrees relative to the $+X$ axis. Since the $10.65 \mathrm{GHz}$ horn was located offset from the focal point of the main reflector (MR), the resulting secondary antenna beam was squinted in the along-scan direction, which resulted in different times that a surface pixel would be viewed by the two feeds. For that reason, the $10.65 \mathrm{GHz}$ receiver azimuthal sampling from -107.97 degrees to 22.03 degrees corresponds to the same measurement swath as the multi-frequency horn. The remaining angles of the rotation were used to view two external calibration targets: the cold target that was Cosmic Microwave Background (CMB) radiation received through the Cold Sky Reflector (CSR) and the hot target that was provided by the blackbody warm load [2]. 


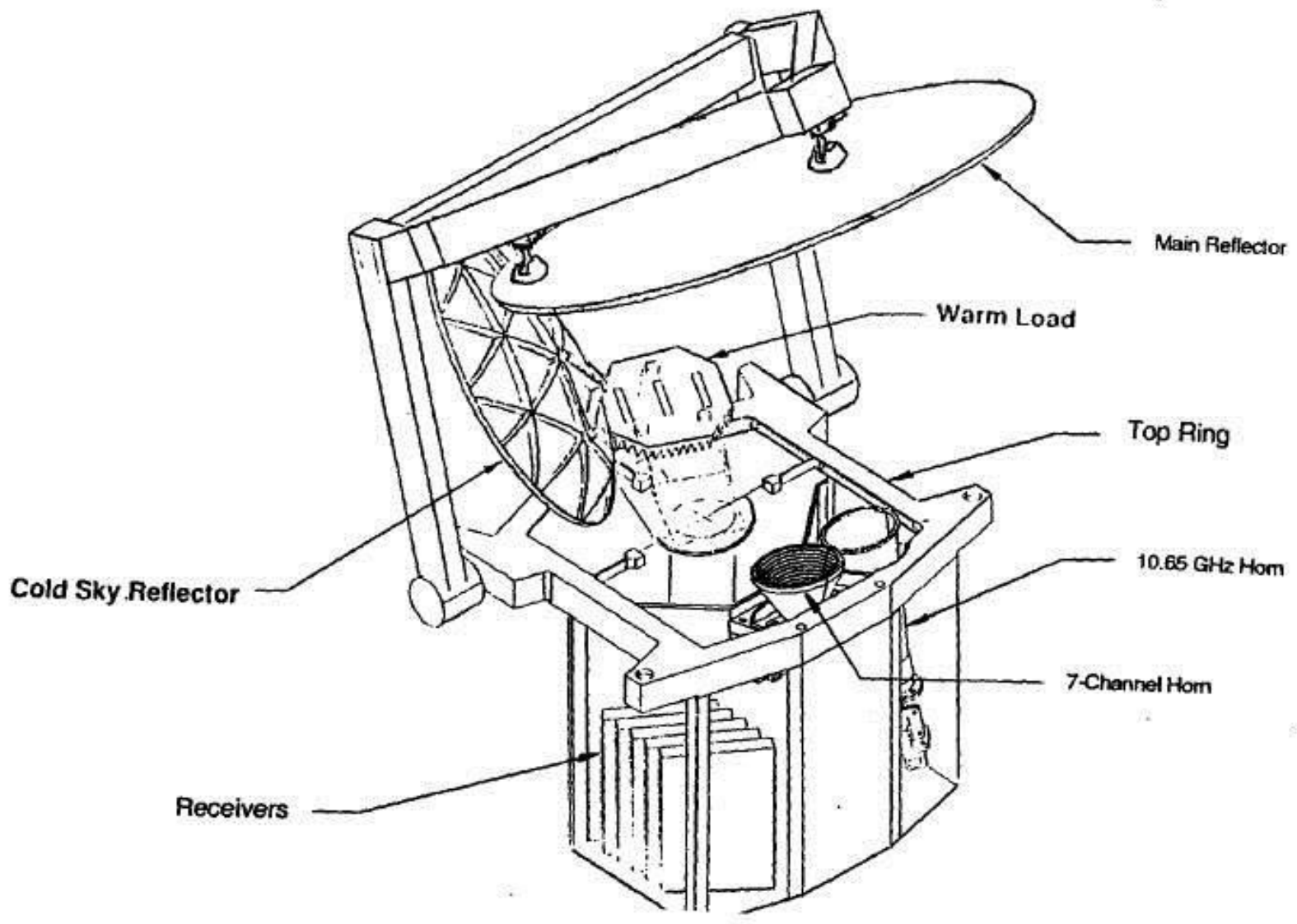

Figure 2.2: Mechanical configuration of the TMI instrument. (source: [2]). 


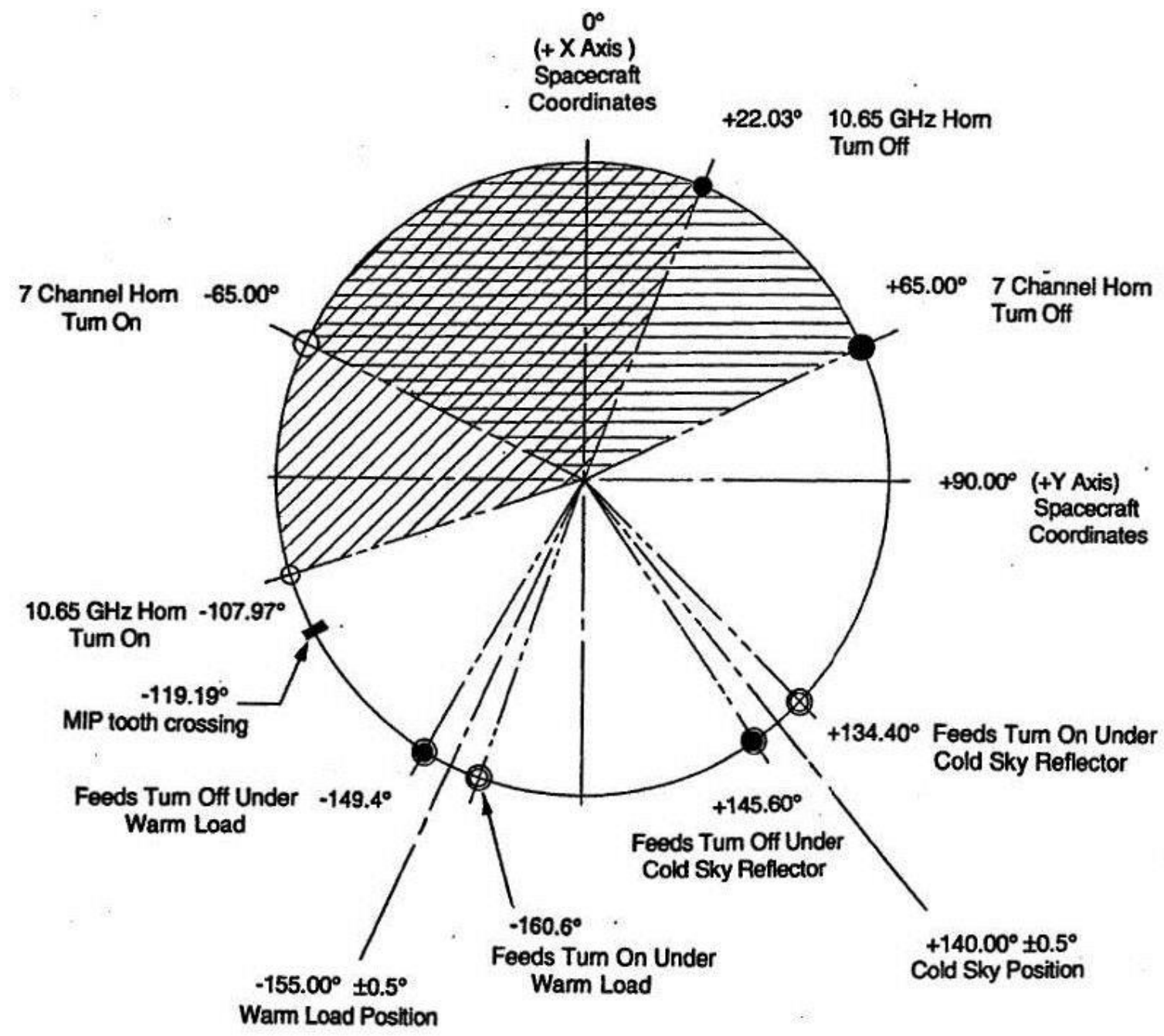

Figure 2.3: TMI's feed horns sampling based on the azimuth rotation angle. (source: [2]).

\subsection{Radiometric Calibration}

\subsubsection{Two-points Calibration:}

TMI was a total power radiometer that used an external two-point calibration method, whereby the feed horn was spinning and passed beneath three brightness temperature $(\mathrm{Tb})$ scenes, as illustrated schematically in Fig. 2.4. The cold radiometric calibration point was the Cold Sky Mirror (CSM), which measured the CMB brightness temperature $\left(T_{c}\right)$ that ranges from $2.73 \mathrm{~K}$ to 
$3.2 \mathrm{~K}$ depending on frequency. The second warm radiometric calibration point was the blackbody hot load target, with a brightness temperature $\left(T_{h}\right)$ equal to its physical temperature measured by using Precision Resistive Temperature (PRT) sensors. After viewing these two calibration points, it is possible to calculate the radiometer transfer function (see Figure 2.5) using linear regression that passes through the hot and cold calibration points $[3,4]$.

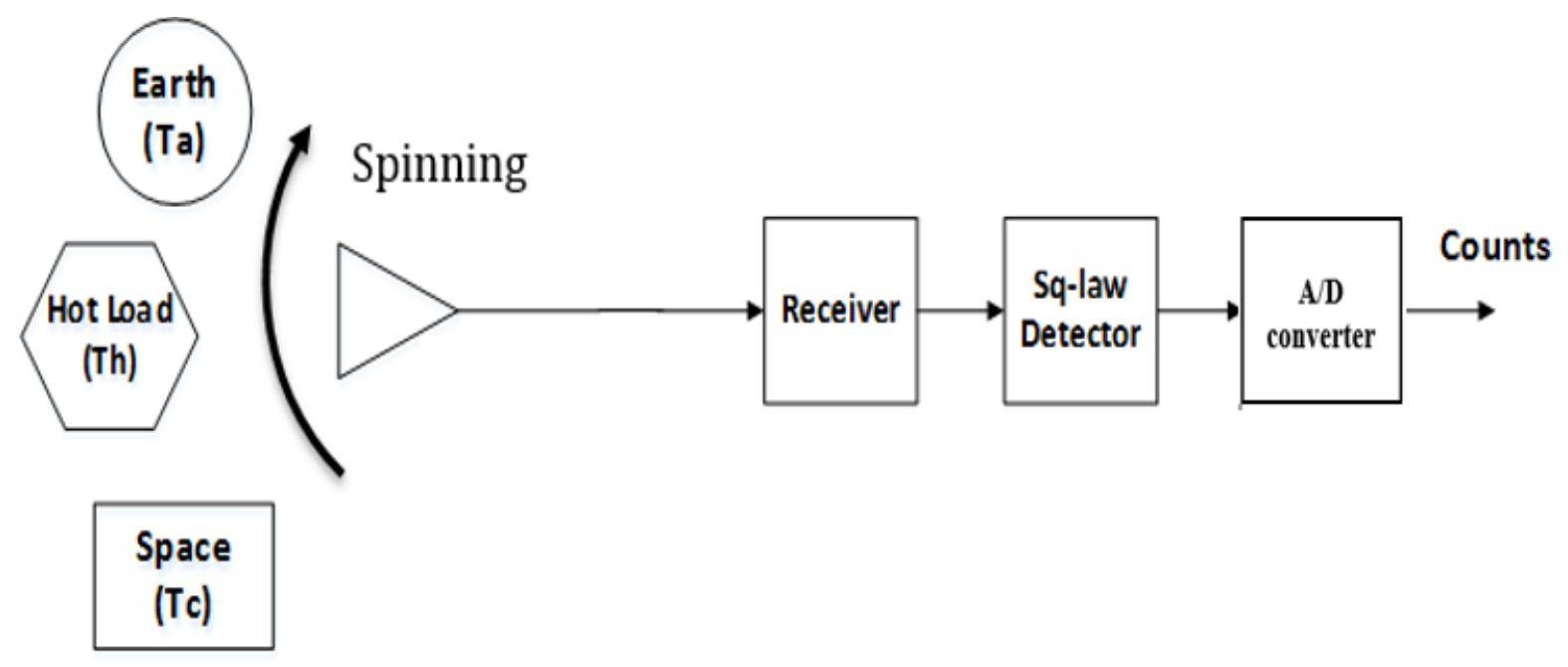

Figure 2.4: Block diagram of the two-point calibration for a TMI.

The slope corresponds to the radiometer system gain:

$$
\text { slope }=\frac{C_{h}-C_{c}}{T_{h}-T_{c}}
$$

and the intercept corresponds to the receiver output (counts) for the input receiver noise temperature:

$$
y_{\text {Intercept }}=C_{c}-\text { slope } * T_{c}
$$

where $C_{h}$ and $C_{c}$ are the radiometer output counts, when the feed views the cold sky mirror and hot load respectively. 


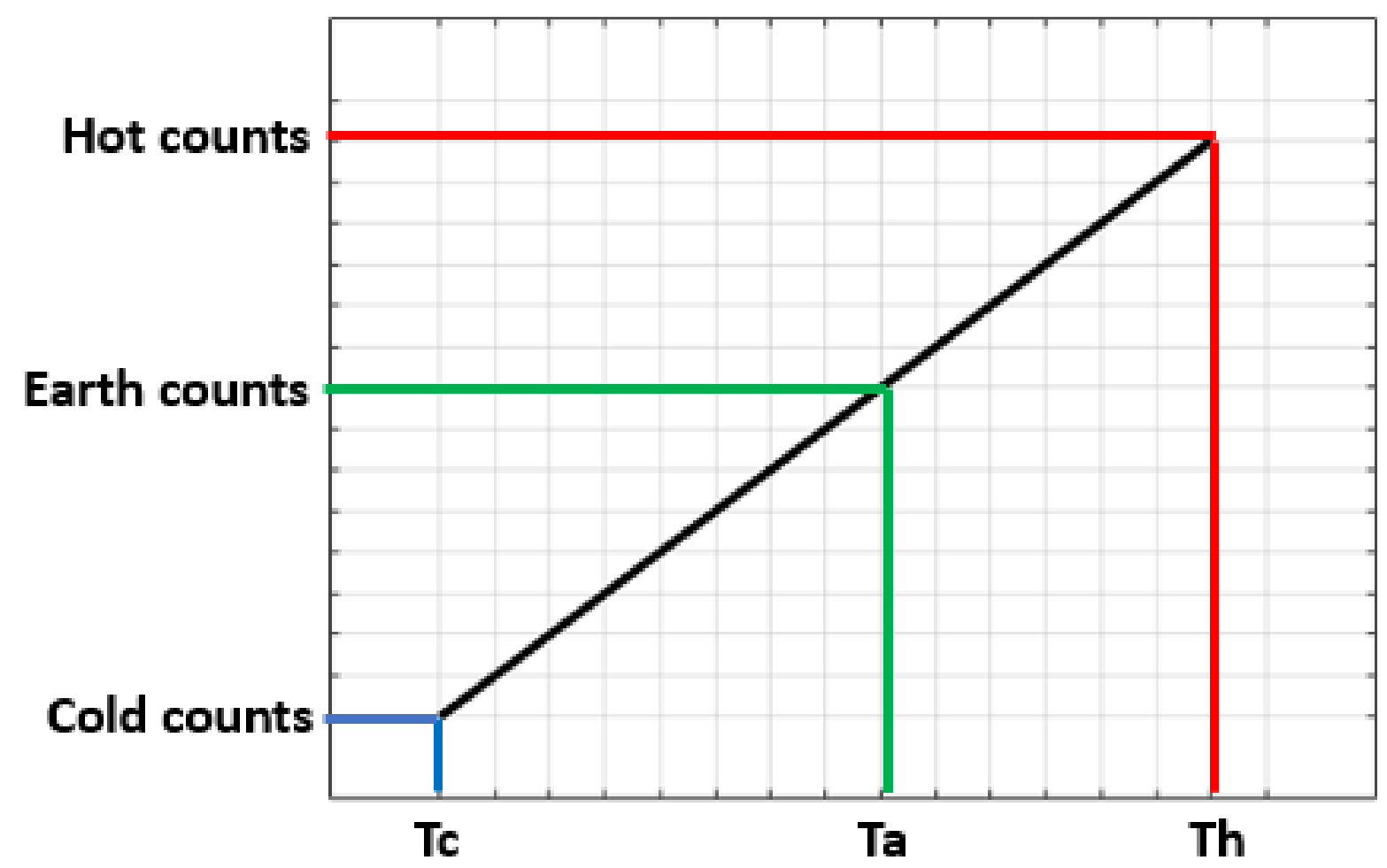

Figure 2.5: The radiometer transfer function from the two-point calibration.

To calculate the unknown scene $T_{a}$, the inverse transfer function (counts to $T_{a}$ ) must be used. Therefore, the antenna temperature of earth can be expressed as:

$$
T_{a}=\frac{1}{\text { slope }} * C_{e}-\frac{y_{\text {intercept }}}{\text { slope }}
$$

where $C_{e}$ are the radiometer output counts from earth.

Converting Equation (2.3) to the standard linear equation yields:

$$
\begin{aligned}
& T_{a}=\text { Gain } * C_{e}+\text { Offset } \\
& \text { Gain }=\frac{1}{\text { slope }}=\frac{T_{h}-T_{c}}{C_{h}-C_{c}}
\end{aligned}
$$




$$
\text { Offset }=\frac{-y_{\text {intercept }}}{\text { slope }}=T_{c}-\text { Gain } * C_{c}
$$

These are the equations that are used in the TMI data processing algorithm (1B11) to convert radiometer output counts to antenna (radiometric) temperature (see TMI Algorithm Theoretical Basis Document [4]).

\subsubsection{Along Scan Calibration Error:}

Each low-resolution TMI scan, which covers the frequencies from $10.65 \mathrm{GHz}$ to $37 \mathrm{GHz}$, consisted of 104 scan positions (pixels), and the high-resolution scan had 208 scan positions, which were the $89 \mathrm{GHz}$ channels. These measurements are desired to be independent of the scan position; however, based upon both deep space and ocean observations, Wentz [5] determined that along scan $\mathrm{Tb}$ biases existed for all channels that were constant with time but variable with scan position.

Later an analysis was performed [6], and an example of the $10.65 \mathrm{GHz} \mathrm{V}$-pol channel brightness temperature anomaly (average Tb per scan pixel minus the average $\mathrm{Tb}$ over all scan positions) is shown in Fig. 2.6 for both ocean and land observations. The major difference between theses curves occurs at the end of the scan positions, where the dependency on the observed temperature is seen. The TMI 1B11 version 7 used only the ocean observation for calculating the along scan correction, but in TMI 1B11 version 8, the scan bias correction will depend linearly on both the ocean and land observations as shown in Equation (2.7). 


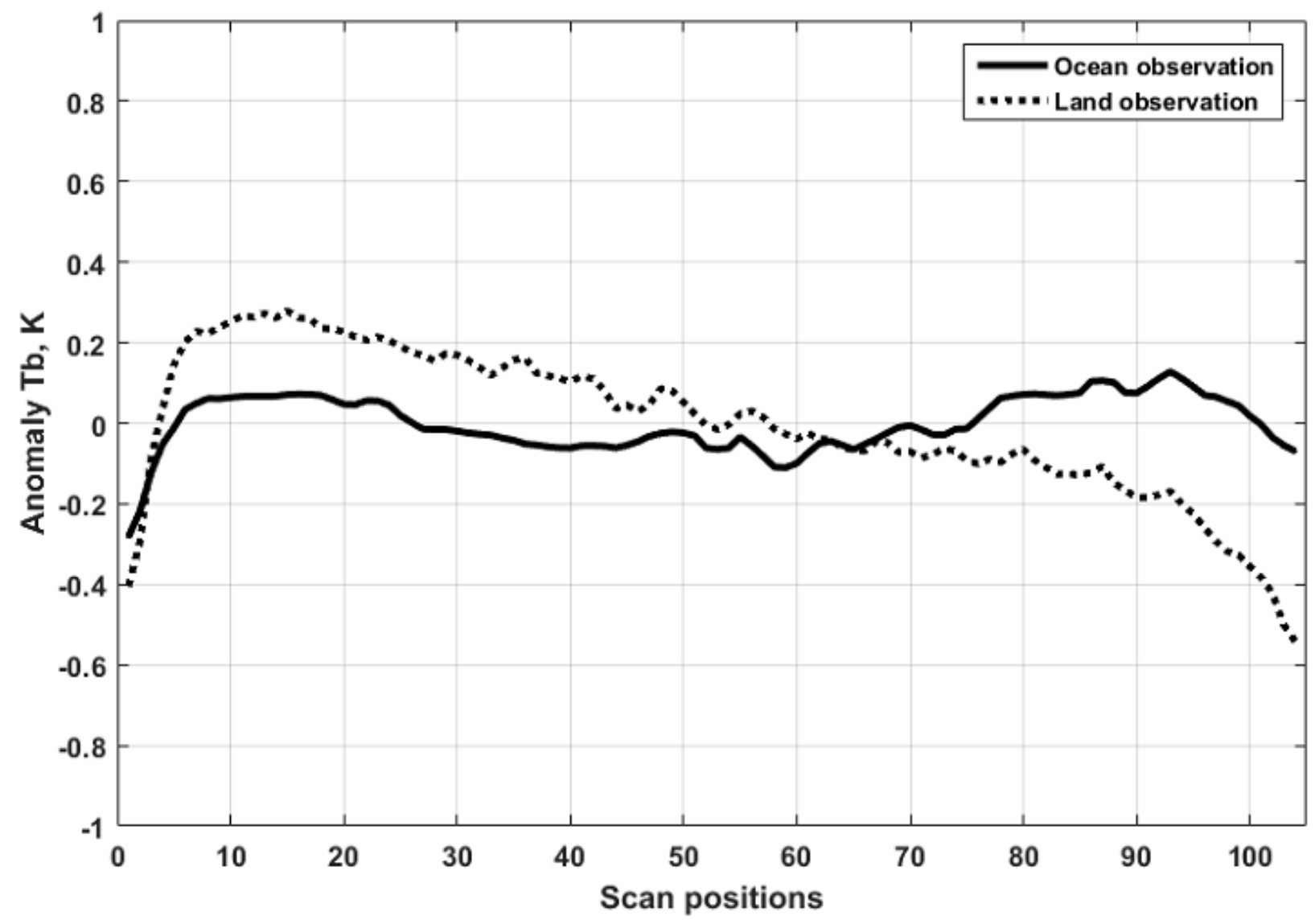

Figure 2.6: Example of Scan bias correction for channel (10.65 GHz, V. pol) for Ocean and land observations.

$$
\text { scan_bias }_{i, j}=m_{i, j} * T_{a_{i, j}}+b_{i, j}
$$

where $m$ and $b$ are the scan bias coefficients that are calculated for each channel $i$ and scan position $j$ (see Appendix A for more details). The along scan error will be corrected in the antenna temperature using the following equation:

$$
\overline{T_{a_{l, j}}}=T_{a_{i, j}}-\text { scan_bias }_{i, j}
$$

where $\overline{T_{a_{l, j}}}$ is the antenna temperature after applying the scan bias correction. 


\subsection{3: Antenna Pattern Correction (APC):}

Because the measured $T_{a}$ was the convolution of the main reflector antenna-pattern with the brightness temperature over a spherical surface, it was necessary to apply an antenna pattern correction (APC) to yield the desired earth scene brightness. The APC depends on the cross polarization $(\chi)$ and the spill-over $\left(\eta_{S}\right)$ coefficients, and these coefficients were measured for each channel prior to launch (see Table 2.2) [7].

Table 2.2: The pre-launch cross polarization and spill-over coefficients.

\begin{tabular}{|c|c|c|}
\hline Channel & Cross polarization $(\chi)$ & Spill-over $(\eta \mathbf{s})$ \\
\hline 10.65 GHz (V. polarization) & 0.00367 & 0.0160 \\
\hline 10.65 GHz (H. polarization) & 0.00459 & 0.0160 \\
\hline 19.35 GHz (V. polarization) & 0.00431 & 0.0218 \\
\hline 19.35 GHz (H. polarization) & 0.00452 & 0.0225 \\
\hline 21.30 GHz (V. polarization) & 0.00577 & 0.0243 \\
\hline 37.00 GHz (V. polarization) & 0.02385 & 0.0125 \\
\hline 37.00 GHz (H. polarization) & 0.01856 & 0.0123 \\
\hline 85.50 GHz (V. polarization) & 0.02003 & 0.0121 \\
\hline 85.50 GHz (H. polarization) & 0.02934 & 0.0108 \\
\hline
\end{tabular}

The earth brightness temperature was received through the main reflector via the feed horn. Thus, the main reflector secondary antenna pattern was required to calculate the beam efficiency [3]. The main beam efficiency is calculated by: 


$$
\eta_{M}=\frac{\int_{0}^{2 \pi} \int_{0}^{\theta_{M B}} F(\theta, \phi) d \theta d \phi}{\int_{0}^{2 \pi} \int_{0}^{\pi} F(\theta, \phi) d \theta d \phi}
$$

where $\eta_{M}$ is the main beam efficiency, $\theta_{M B}$ is the main beam angle (defined as $2.5^{*} \mathrm{HPBW}$ ), and the $F(\theta, \phi)$ is the antenna pattern. By the conservation of energy, the spillover efficiency is calculated using Equation (2.10) and illustrated in Figure 2.7. Since the spill-over beam $\left(\eta_{S}\right)$ views space during the normal operation, the total observed temperature is given by Equation (2.11) [8].

$$
\begin{gathered}
\eta_{S}=1-\eta_{M B} \\
\overline{T_{a l}}=\left(1-\eta_{S_{i}}\right) * T_{\text {scene }_{i}}+\eta_{S_{i}} * T_{c}
\end{gathered}
$$

where $\overline{T_{a l}}$ is the TMI observed antenna temperature after removing the along scan bias error for each channel $i, T_{\text {scene }}$ is the Earth scene temperature. To calculate the scene temperature, Equation (2.11) can be derived as:

$$
T_{\text {scene }_{i}}=\frac{\overline{T_{a l}}-\eta_{S_{i}} * T_{c}}{\left(1-\eta_{S_{i}}\right)}
$$




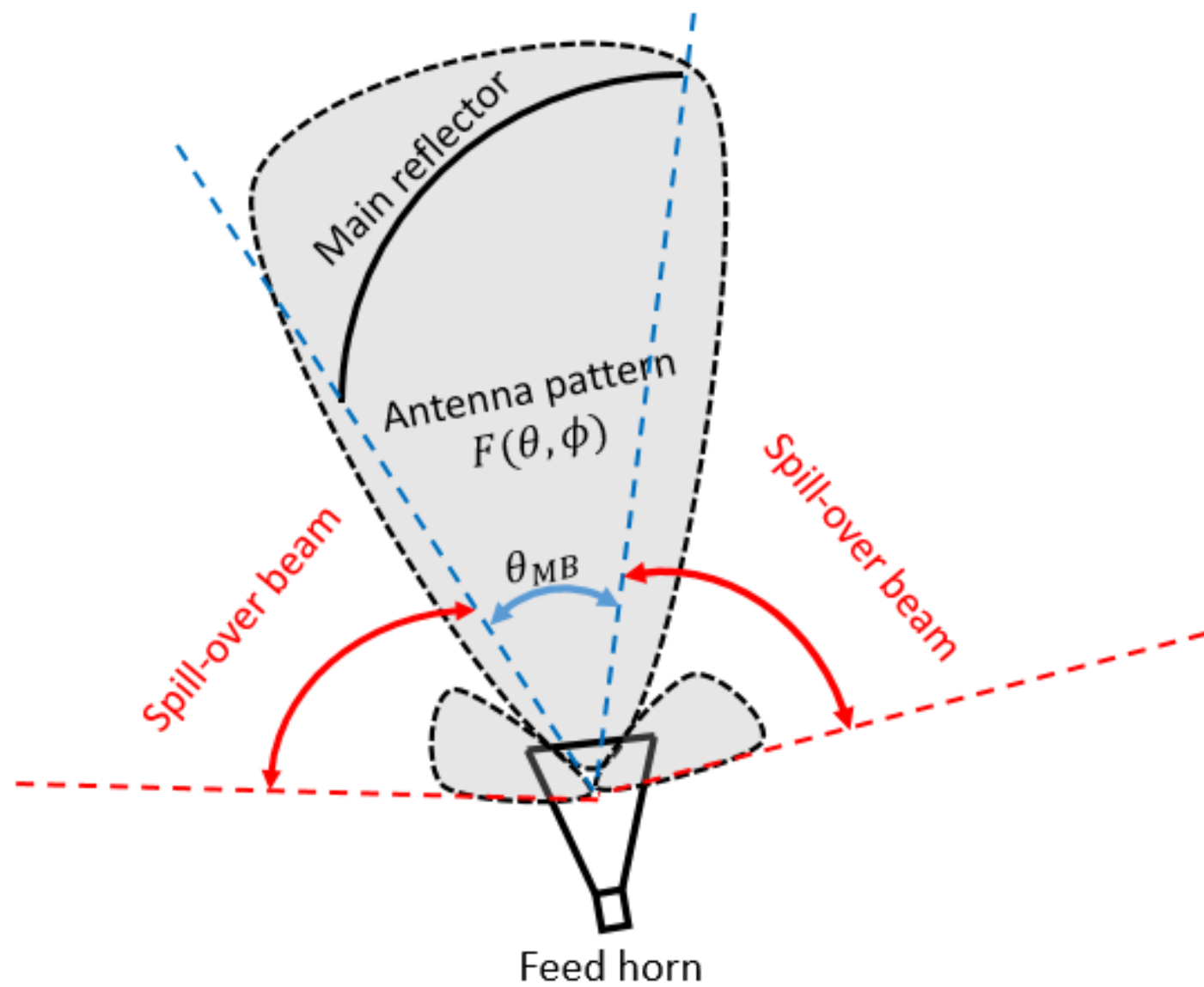

Figure 2.7: Definition of spill-over beam pattern relative to the feed horn primary pattern and the interception of the main reflector.

Although the spill-over component of brightness has been removed from the observed temperature, the scene temperature still has a cross-polarization component of brightness that must be removed. Thus, following the conservation of energy, resulting polarized brightness (for channels 10.65 GHz V \& H) are given in Equations (2.13) and (2.14) respectively.

$$
T_{\text {scene }_{10.65 V}}=\left(1-\chi_{10.65 \mathrm{~V}}\right) * T_{\text {scene }, \mathrm{V}}+\chi_{10.65 \mathrm{~V}} * T_{\text {scene }, \mathrm{H}}
$$




$$
T_{\text {scene }_{10.65 H}}=\left(1-\chi_{10.65 H}\right) * T_{\text {scene }, H}+\chi_{10.65 H} * T_{\text {scene }, V}
$$

where $\chi$ is the antenna pattern cross-polarization coefficient.

Using the above equations to find the $T_{\text {scene, } V}$ and $T_{\text {scene,H }}$ is applicable for all channels except channel $21.3 \mathrm{~V}$, which doesn't have the horizontal polarization measurement. However, it is possible to estimate the horizontal scene brightness from the measured vertical $T_{\text {scene, } V}$ through correlation. To determine the cross-correlation, the modeled temperature was calculated using the Radiative Transfer Model (RTM) over the full dynamic range of ocean/atmospheric environmental conditions, and the results are presented as a scatter diagram in Fig. 2.8. It is apparent that the horizontal brightness can be estimated from a simple linear relationship

$$
T_{\text {scene-21.3H-modeled }}=1.6634 * T_{\text {scene-21.3V-modeled }}-195.36
$$

Thus, for all TMI channels, Equation (2.16) is used to convert the antenna temperature (after removing the along scan error) into main beam brightness temperature:

$$
T_{b, i}=C_{i} \overline{T_{a, l}}-D_{i} \overline{T_{a, l}^{*}}-E_{i}
$$

where $T_{b}$ was the scene brightness temperature of the $i^{\text {th }}$ channel, $T_{a}$ was the measured antenna brightness temperature, $T_{a}{ }^{*}$ was the corresponding measured cross polarized antenna temperature, and $C_{i}, D_{i}$, and $E_{i}$ were the corresponding channel dependent antenna pattern correction coefficients as listed in Table 2.3 (See Appendix B for the detailed derivation), which are coefficients derived are for the TMI 1B11 V8. It should be noted that previously $1 \mathrm{~B} 11 \mathrm{~V} 7$ used the SSM/I APC coefficients. 


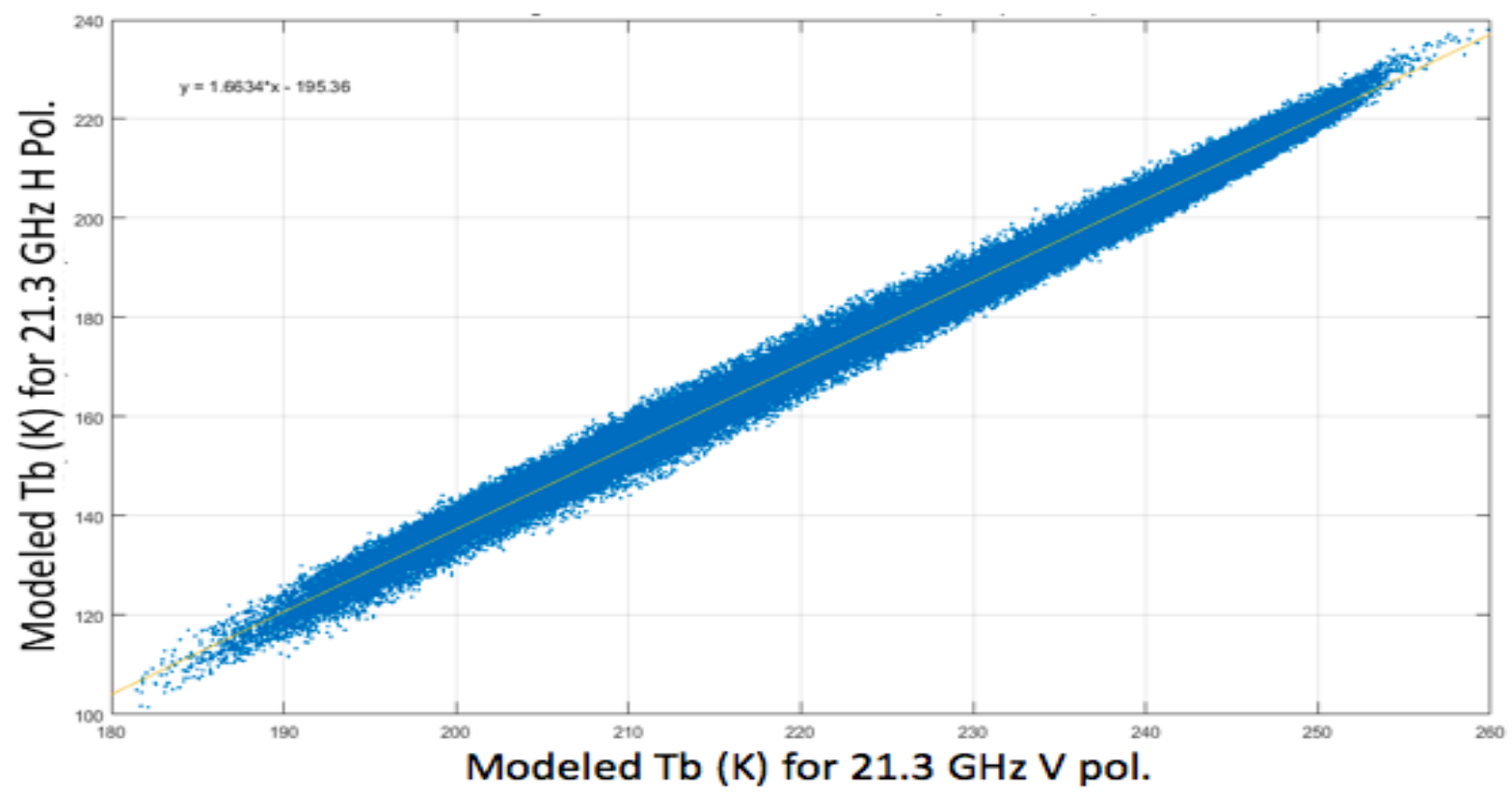

Figure 2.8: The ratio between the horizontal and vertical polarization of the modeled $\mathrm{Tb}$ for frequency $21.3 \mathrm{GHz}$.

Table 2.3: TMI's antenna pattern correction (APC) coefficients

\begin{tabular}{|c|c|c|c|}
\hline $\mathbf{i}$ & $\mathbf{C}_{\mathbf{i}}$ & $\mathbf{D}_{\mathbf{i}}$ & $\mathbf{E}_{\mathbf{i}}$ \\
\hline $\mathbf{1 0 . 6 5} \mathbf{V}$ & 1.02002 & 0.00376 & 0.04439 \\
\hline $\mathbf{1 0 . 6 5} \mathbf{H}$ & 1.02096 & 0.00470 & 0.04439 \\
\hline $\mathbf{1 9 . 3 5} \mathbf{V}$ & 1.02673 & 0.00445 & 0.06083 \\
\hline $\mathbf{1 9 . 3 5} \mathbf{H}$ & 1.02768 & 0.00466 & 0.06285 \\
\hline $\mathbf{2 1 . 3 0} \mathbf{V}$ & 1.02100 & 0 & -1.05520 \\
\hline $\mathbf{3 7 . 0 0} \mathbf{~ V}$ & 1.03788 & 0.02522 & 0.03457 \\
\hline $\mathbf{3 7 . 0 0} \mathbf{H}$ & 1.03208 & 0.01963 & 0.03399 \\
\hline $\mathbf{8 5 . 5 0} \mathbf{V}$ & 1.03358 & 0.02130 & 0.03351 \\
\hline $\mathbf{8 5 . 5 0} \mathbf{~ H}$ & 1.04212 & 0.03124 & 0.02969 \\
\hline
\end{tabular}




\section{$\underline{2.3 \mathrm{TMI} 1 \mathrm{~B} 11 \mathrm{~Tb} \text { Product }}$}

The TMI counts to $\mathrm{Tb}$ algorithm (1B11) [9] converts the radiometer output counts to polarized antenna temperature by using the inverse transfer function and other corrections. After that, the antenna pattern correction is applied to produce brightness temperatures ( $\mathrm{Tb})$. When any of TRMM's instruments data were modified, the various TRMM products are updated and reprocessing occurs for the entire dataset, and at this time the products are given version \#'s.

Although the TMI observations have been corrupted by the emissive main reflector from the initial on-orbit start-up, the first emissive correction was applied in 2001, which is called 1B11 v6. The procedure of this version, which is illustrated in Figure 2.9, started by calculating the gain and offset, using the inverse transfer function of the cold and hot counts with the hot load measured temperature. After calculating the antenna temperature, using the linear inverse transfer function (calculated gain and offset) with earth counts, the APC was applied to calculate the brightness temperature. However, the APC coefficients were based on the design heritage instrument, which is SSMI. Finally, the emissive reflector was corrected, but with an ad-hoc correction, the reflector emissivity coefficients were derived based on the SSMI measurements, while the physical temperature of the main reflector was incorrectly assumed constant, based on the orbit average.

Since the physical temperature of the main reflector was changing based on the sun coordinate, the 1B11 v6 did not provide the good radiometric calibration. Thus, in 2006, based upon work at CFRSL [10] another version was developed, which was called 1B11 v7. This version was an upgrade of version 6 as shown in Fig. 2.10. It removed the erroneous constant physical temperature assumption of version 6, and introduced a time-varying Tb bias correction look-up table that depends on the sun coordinates, which represented by solar beta angle and time since 
eclipse. While the brightness temperatures of version 7 were a significant improvement from version 6; nevertheless, both version 6 and 7 were based on strictly empirical ad-hoc corrections. Therefore, in this dissertation, the new version, known as 1B11 v8, will be described (see Figure 2.11), which models Tb corrections using rigorous physical principles e.g., the emissive reflector $\mathrm{Tb}$ correction based upon radiative transfer theory. Instead of providing a statistical representation of the time varying $\mathrm{Tb}$ bias (from V7), the emissivity coefficients are measured using deep space calibrations (DSC) and the time varying main reflector physical temperature is estimated using the single difference between measured and theoretical Tb's at only $10.6 \mathrm{GHz} \mathrm{V}$ pol. Other parts of the algorithm are similar to $v 7$, except pre-launch and on-orbit measurements are used to estimate the model coefficients. Finally, this algorithm is based upon the careful analysis of the entire 17-year history of TMI measurements. During this process, several important discoveries were made (e.g., cold load RFI and hot load sun intrusion) and corrections were implemented to mitigate these random anomalies. Also, this algorithm uses APC based on TMI measurements. 


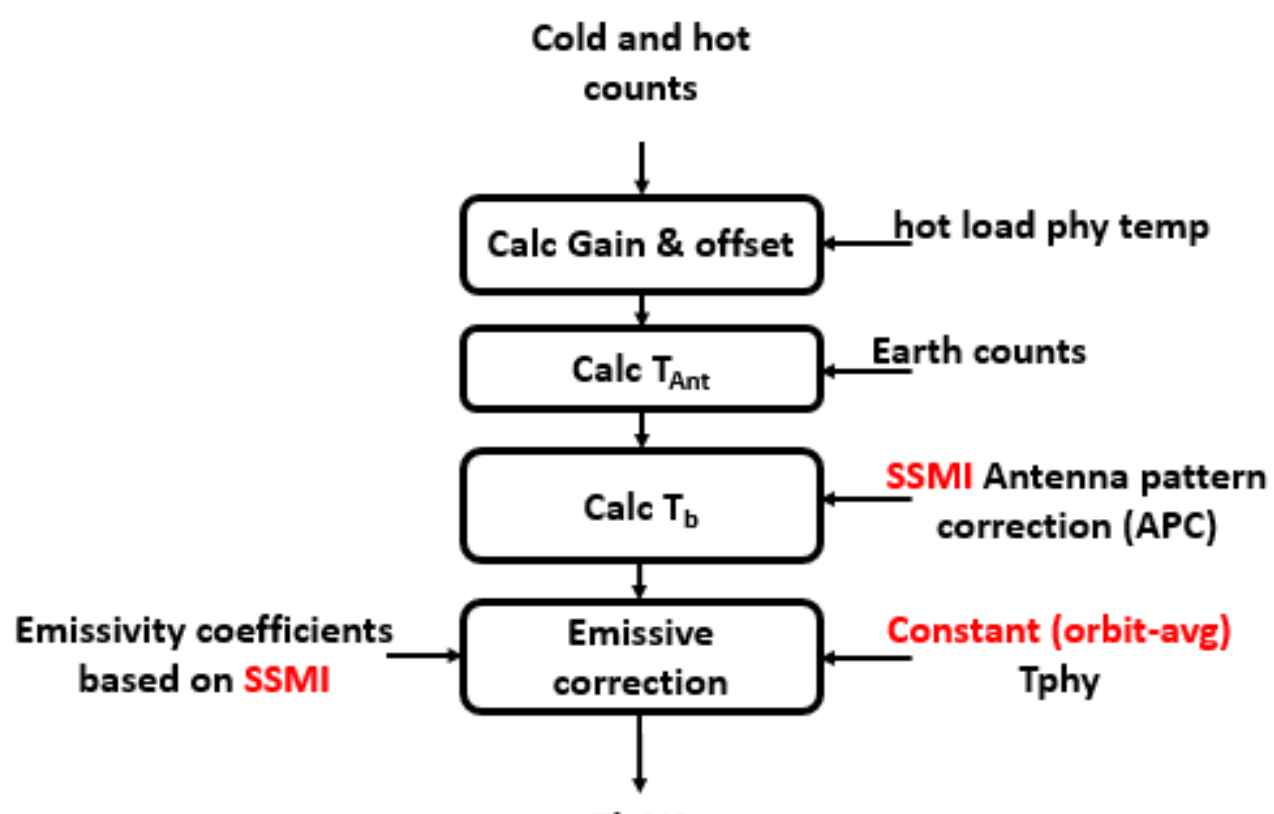

Tb V6

Figure 2.9: Flow chart of the TMI 1B11 v6 procedure.

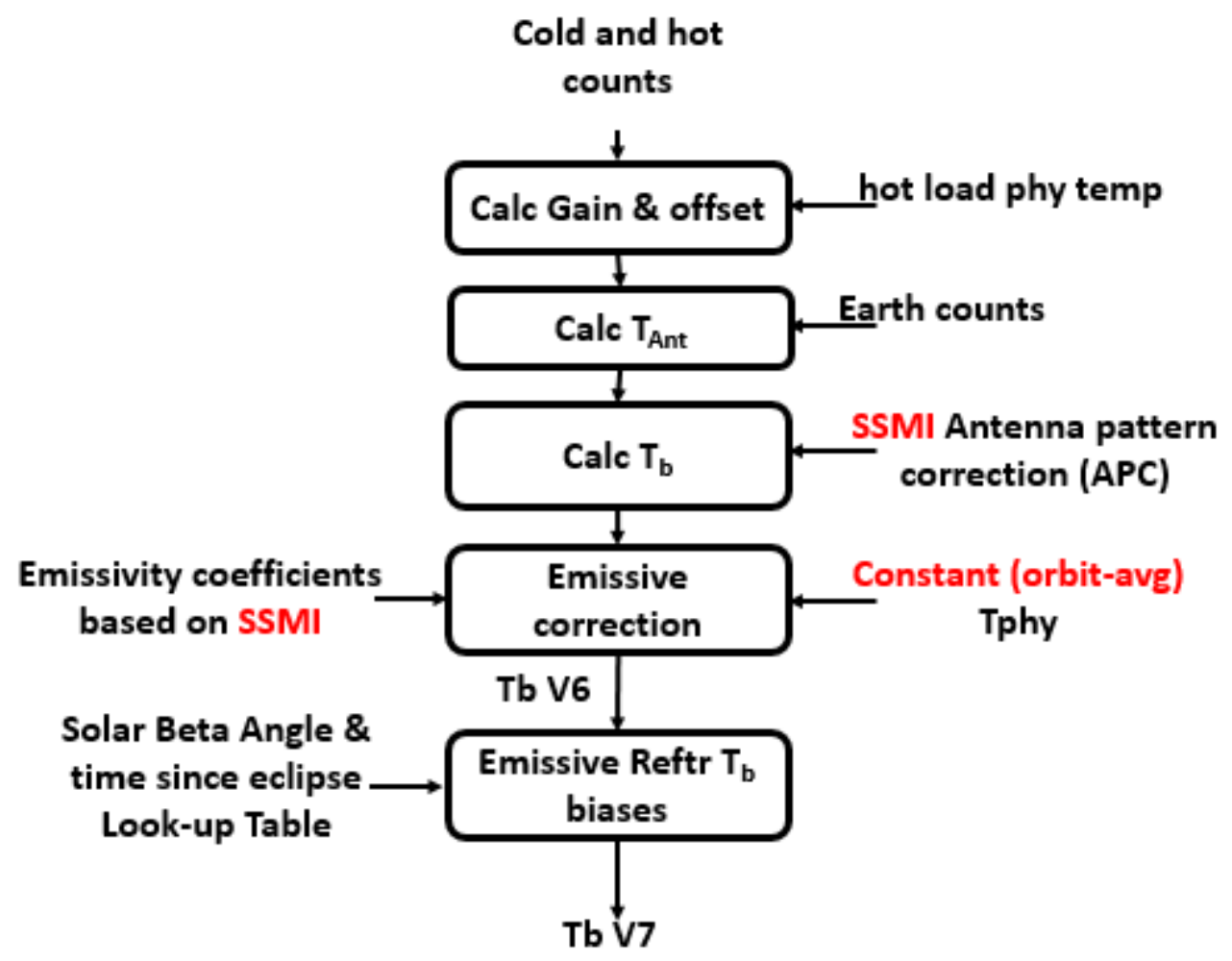

Figure 2.10: Flow chart of the TMI 1B11 v7 procedure. 


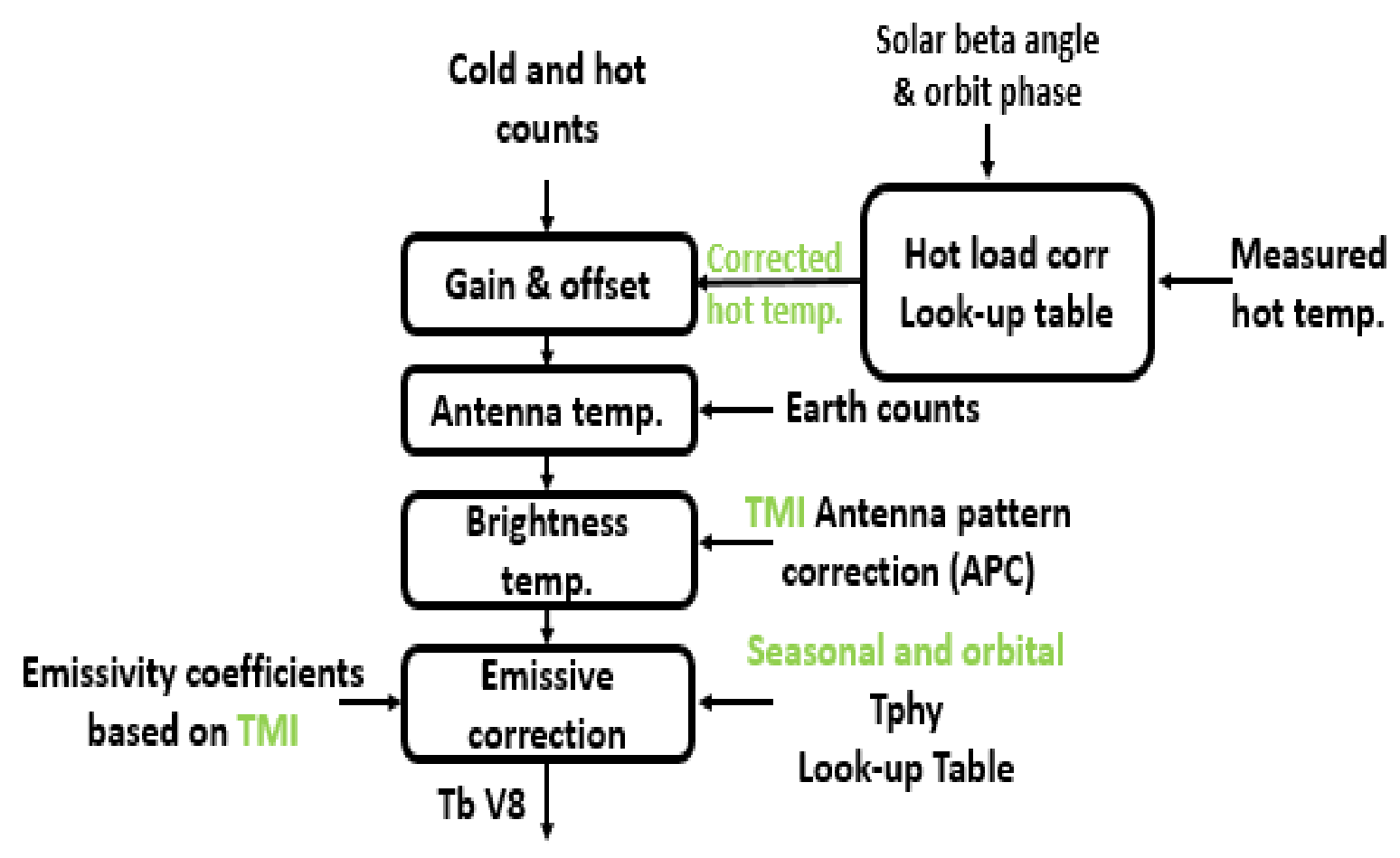

Figure 2.11: Flow chart of the TMI 1B11 v8 procedure.

\section{$\underline{2.4 \text { Conclusion }}$}

Although all space-borne conical scanning microwave radiometers have undergone extensive ground test and calibration before launch, it is usual that there are unexpected calibration issues experienced once on-orbit. It is impossible to duplicate the on-orbit environment in ground testing, and further, calibration is performed at the feed level (without the main reflector). Thus, it is customary that a period of on-orbit calibration/validation $(\mathrm{Cal} / \mathrm{Val})$ be performed to characterize the radiometer transfer function. Fortunately, once properly calibrated, radiometers have been found to be extremely stable.

This was the case for TMI, where a major calibration issue was discovered during on-orbit checkout. The issue was that the ocean scene brightness temperature was too warm by almost 10 
K. After significant testing, including a deep space calibration, it was discovered that the main reflector was slightly emissive, although the root cause was not determined. The TMI 1B11 countsto- $\mathrm{Tb}$ processing software were modified to remove the yearly mean $\mathrm{Tb}$ bias, and for almost 10 years this fix was used.

However, in 2007, the orbital cycle Tb bias, which was caused by the slightly emissive main reflector, was removed. This empirical procedure used single differences between the observed and modeled Tb's to produce an ad-hoc correction, which was a Tb bias look-up table correction generated based on the solar coordinates. This procedure resulted in the TMI 1B11 V7 brightness temperature product, and although the results were considerably improved, they were strictly statistical and ad hoc.

From a science prospective, it is important that the 17-year legacy TMI brightness temperature product have a rigorous, transparent, theoretical basis. Thus, the goal of this dissertation is to develop a new radiometer counts to $\mathrm{Tb}$ algorithm that is based upon fundamental electromagnetic theory. The Tb product that uses this improved algorithm is known as the TMI 1B11 V8. The development of this algorithm will be discussed in detail in Chapter 4.

\section{$\underline{2.5 \text { References }}$}

[1] C. Kummerow, W. Barnes, T. Kozu, J. Shiue, and J. Simpson, "The tropical rainfall measuring mission (TRMM) sensor package," Journal of atmospheric and oceanic technology, vol. 15, no. 3, pp. 809-817, 1998.

[2] "Tropical Rainfall Measuring Mission Microwave Imager (TMI): Critical Design Review," Hughes, Sept 1993, unpublished. 
[3] F. T. Ulaby et al., Microwave radar and radiometric remote sensing (Vol. 4, No. 5, p. 9596 \& 272-277). University of Michigan Press Ann Arbor, 2014.

[4] Y. Ji, S. Bilanow, and E. Stocker, “Algorithm Theoretical Basis Document (ATBD) for NASA TRMM TMI Level 1B Algorithm,” TRMM Science and Data Information System (TSDIS), Version 1, 2008.

[5] F. J. Wentz, P. Ashcroft, and C. Gentemann, "Post-launch calibration of the TRMM microwave imager," IEEE Transactions on Geoscience and Remote Sensing, vol. 39, no. 2, pp. 415-422, 2001.

[6] R. Kroodsma, S. Bilanow, Y. Ji, and D. McKague, "TRMM Microwave Imager (TMI) Updates for Final Data Version Release," Proc. IEEE International Geoscience and Remote Sensing (IGARSS), Fort Worth, USA, July 23-28, 2017, accepted for publication.

[7] F. J. Wentz, "A 17-yr climate record of environmental parameters derived from the Tropical Rainfall Measuring Mission (TRMM) Microwave Imager," Journal of Climate, vol. 28, no. 17, pp. 6882-6902, 2015.

[8] D. Draper, "Calibration Data Book for Global Precipitation Measurement (GPM) Microwave Imager (GMI), Ball Aerospace \& Technologies Corp," ed: Tech. Rep No: 2344649, RevH, 2016.

[9] TRMM Microwave Imager (TMI) Level 1B Calibrated Brightness Temperature (TB) product available at: https://disc.gsfc.nasa.gov/precipitation/documentation/TRMM_README/TRMM_1B11 _readme.shtml 
[10] K. Gopalan, "A Time-Varying Radiometric Bias Correction for The TRMM Microwave Imager," Ph.D. dissertation, Dept. Electrical and Computer Engineering, University of Central Florida, Orlando, FL, 2008. 


\section{CHAPTER 3: HOT LOAD CORRECTION}

F. Alquaied, R. Chen, W. Jones, "Hot load temperature correction for the Tropical Rainfall Measuring Mission Microwave Imager (TMI)", Proc. IEEE International Geoscience and Remote Sensing (IGARSS), Fort Worth, USA, July 23-28, 2017, accepted for publication.

During the derivation of the main reflector physical temperature (described in Chapter-4), it became apparent that another radiometric calibration anomaly (associated with the hot load) existed and needed to be corrected. Under certain solar geometry conditions, the radiometer hot counts did not follow the measured hot load physical temperature as expected. For example, consider a particular orbit presented in Fig. 3.1, where the TMI radiometer output hot counts, measured hot load temperature, radiometer output cold counts, calculated inverse-transfer function gain and inverse transfer function offset are plotted as a function of the orbital solar cycle (phase from orbit midnight) that is proportional to relative time.

First, it is apparent that the cyclic pattern of these parameters is quasi-sinusoidal with a period equal to the orbit period. This suggests that the changes are driven by the physical temperature cycle of the respective TMI radiometer receiver, which is not measured. Since the hot load is external to the radiometer canister (see Fig. 2.2), its measured physical temperature should not match-up with the internal receiver physical temperature. However, the orbital pattern, for both physical temperatures, is driven by the sun thermal loading (during daylight) and the radiative cooling (during night, orbit eclipse). Thus, the true receiver physical temperature should be similar to the measured hot load, but the receiver temperature would lag somewhat (i.e., be shifted to the right slightly out-of-phase, typically by $10-20 \%$ of the orbit period). 
Therefore, given this simplistic thermal model for physical temperature, what makes these observed patterns in Fig 3.1 be anomalous? To answer this question, let's consider previous onorbit history of microwave radiometers.

(a)

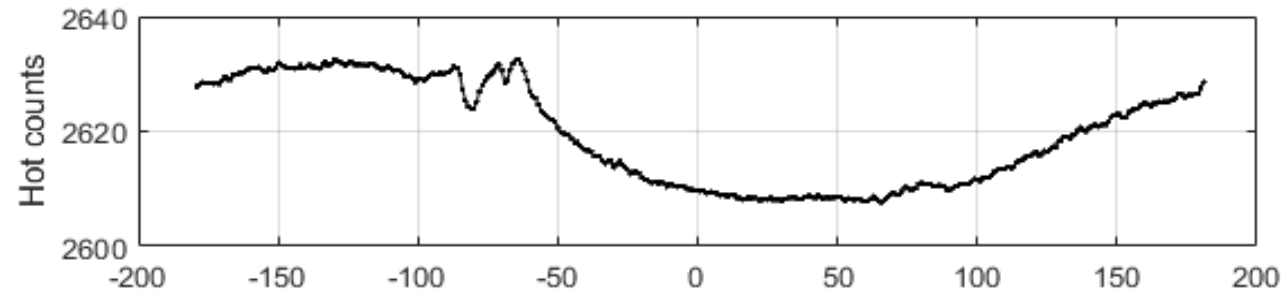

(b)

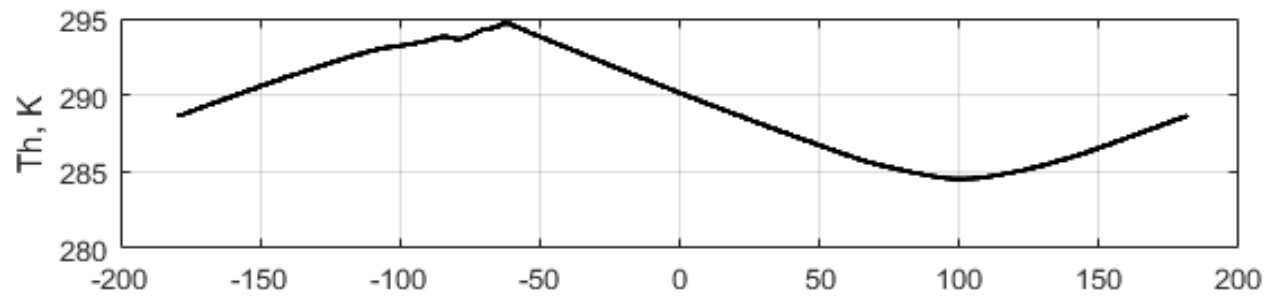

(c)

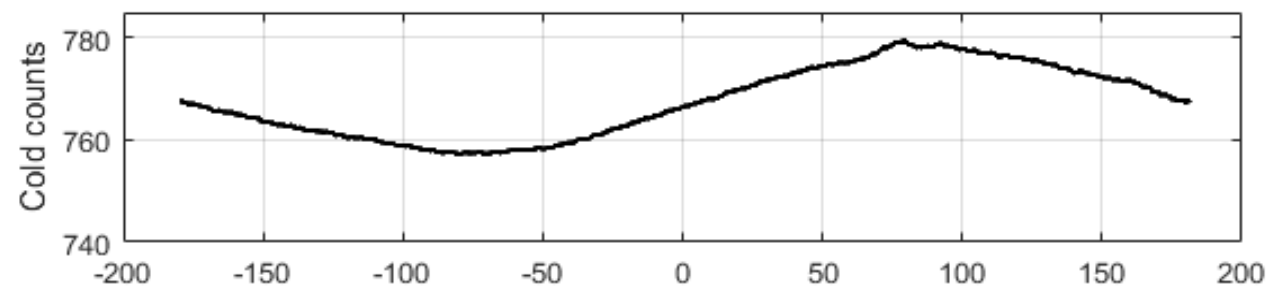

(d)

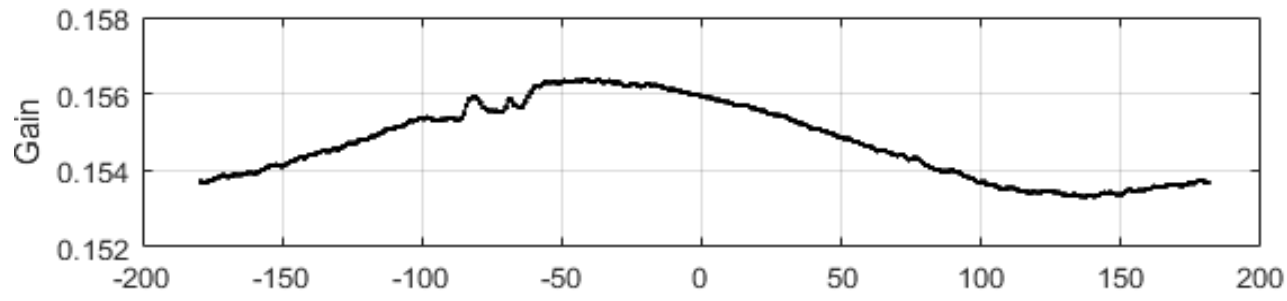

(e)

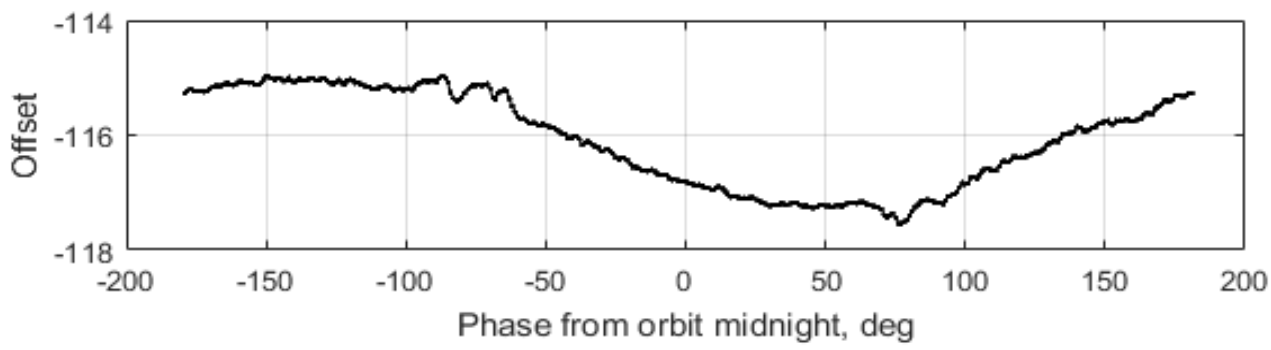

Figure 3.1: TMI parameters for $10.65 \mathrm{~V}$ channel during TRMM orbit \# 87736: (a) Hot counts, (b) measured hot load physical temperature, (c) cold counts, (d) inverse-xfer gain and (e) inverse-xfer offset. 


\subsection{Orbital Receiver Gain Cycle}

Previous on-orbit radiometric calibration experience has indicated that the receiver gain changes based on its physical temperature; thus, according to Ghazi [1], the receiver transfer function gain $\left(G_{r e c}\right)$ of each TMI channel can be modeled as following:

$$
G_{r e c}=G_{0} * g\left(T_{\text {rec-phy }}\right)
$$

where $G_{0}$ is the orbit-mean receiver gain and $g\left(T_{\text {rec-phy }}\right)$ is the orbital gain changes due to the receiver physical temperature, which is approximately sinusoidal with a period of one-orbit and $180^{\circ}$ out-of-phase with the physical temperature. Thus, the increasing physical temperature decreases the receiver gain and vice versa. On the other hand, for the inverse transfer function, the receiver physical temperature and inverse-transfer function gain are in-phase.

For TMI, the CMB brightness is constant over the orbit, therefore the cold counts can be modeled as:

$$
C_{c}=C M B / \text { inverseXfgain }- \text { inverseXfroffset }
$$

where the inverseXfrgain is given in panel-d and the inverseXfr $r_{\text {ofset }}$ is given in panel-e. For a single orbit, note that the dynamic range for the cold counts (panel-c) is 20 and the corresponding dynamic range for the offset is 2 ; therefore, the change in cold counts is dominated by the change in the inverseXfrgain, which is proportional to the receiver physical temperature. Thus, the observed TMI cold counts $\left(C_{c}\right)$ and the delayed hot load physical temperature (shifted to the right by $\sim 50^{\circ}$ ), as a proxy for the unmeasured receiver temperature are in-phase as expected.

On the other hand, consider the inverseXfr $r_{\text {gain }}$ cycle (panel-d), where there is an unexpected "fluctuation" in the gain pattern at the x-axis values from $-90^{\circ}$ to $-70^{\circ}$ orbit phase. Even though 
this perturbation is small, it is nevertheless anomalous. Next examine the hot load measured counts, and note that there is a significant perturbation that occurs at the same point; yet, there is no such perturbation in the hot load physical temperature. This suggests that there is another cause for these perturbations, which we believe to be the sun intrusion heating of the tips of the pyramidal loads that make-up the hot load blackbody target.

\subsection{Sun Intrusion to The Hot Load}

To confirm this hypothesis, evidence of possible sun intrusion into the hot load is required, which is presented in Figure 3.2 (left-hand photo during TMI ground testing). When the feeds were not located beneath the hot load, the pyramidal elements of the hot load were visibly exposed (see red circle). Although the instrument was rotating, the hot load was fixed and could experience sun intrusion over a limited range of solar coordinates.

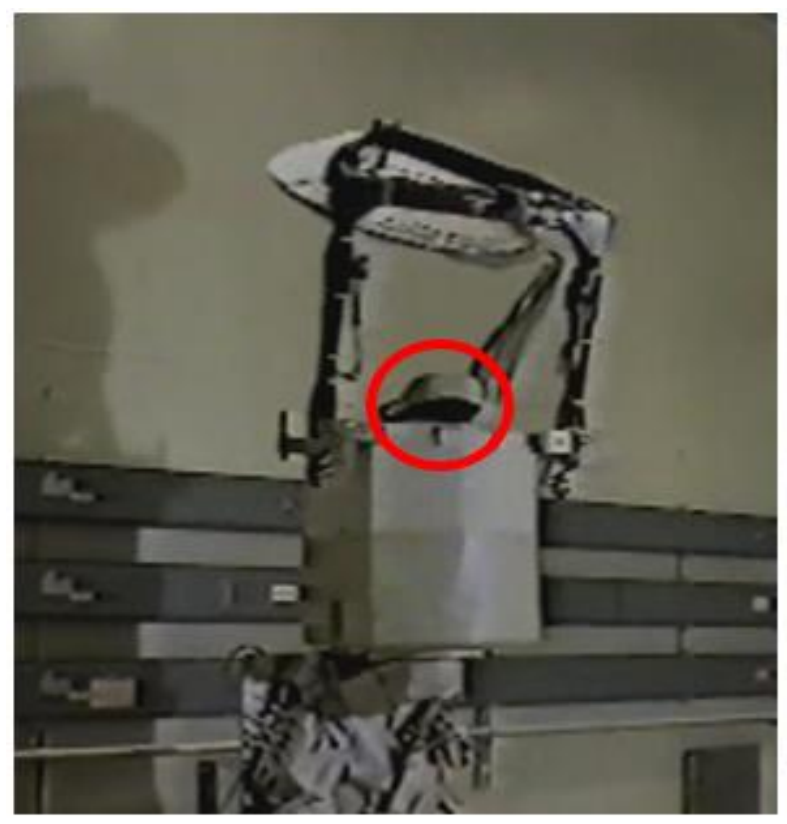

(a)Main reflector looking forward

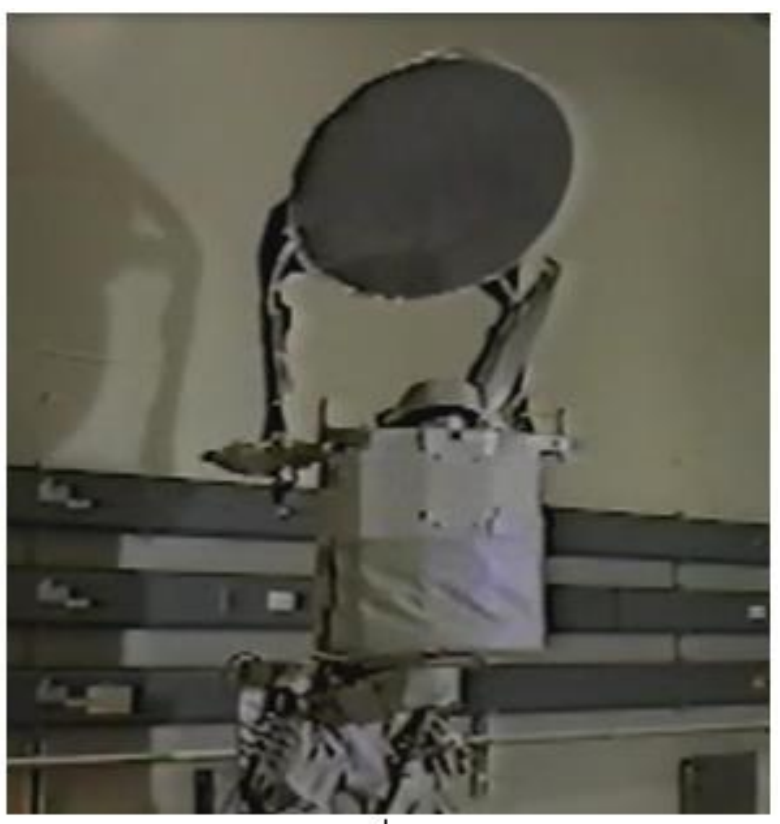

(b) Main reflector looking backward

Figure 3.2: Possible hot load exposure during the ground test of the TMI. (Source: [3]) 
To understand solar intrusion heating of the hot load, consider Fig. 3.3. When the sun lineof-sight passed beneath the cylindrical shield and intersected the hot load pyramids, the tips would be heated by solar energy absorption, which created a thermal gradient from the absorber tips to the pyramid base that contained the PRT physical temperature sensors. As a result, this elevated temperature of the tips resulted in increased blackbody emission brightness temperature captured by the feed horn and the resulting hot load counts (Fig. 3.2 a). Thus, when this occurred, there would be a transient condition, whereby hot counts at the radiometer output would instantaneously increase, but the measured the hot load physical temperature would be unchanged until the thermal energy propagated to the base.

Since the contaminated $C_{h}$ was used in the two-point radiometric calibration and because the measured hot load physical temperature did not match the hot load emission brightness temperature, an error was introduced in the inverse-xfer gain and offset. To correct this radiometric calibration issue, the two factors must be resolved; namely, the solar coordinates relative to TRMM of the sun intrusion and the -Y solar array orientation that occasionally blocked this sun intrusion. In this Chapter, the approach to correct the hot load measurements will be provided. 


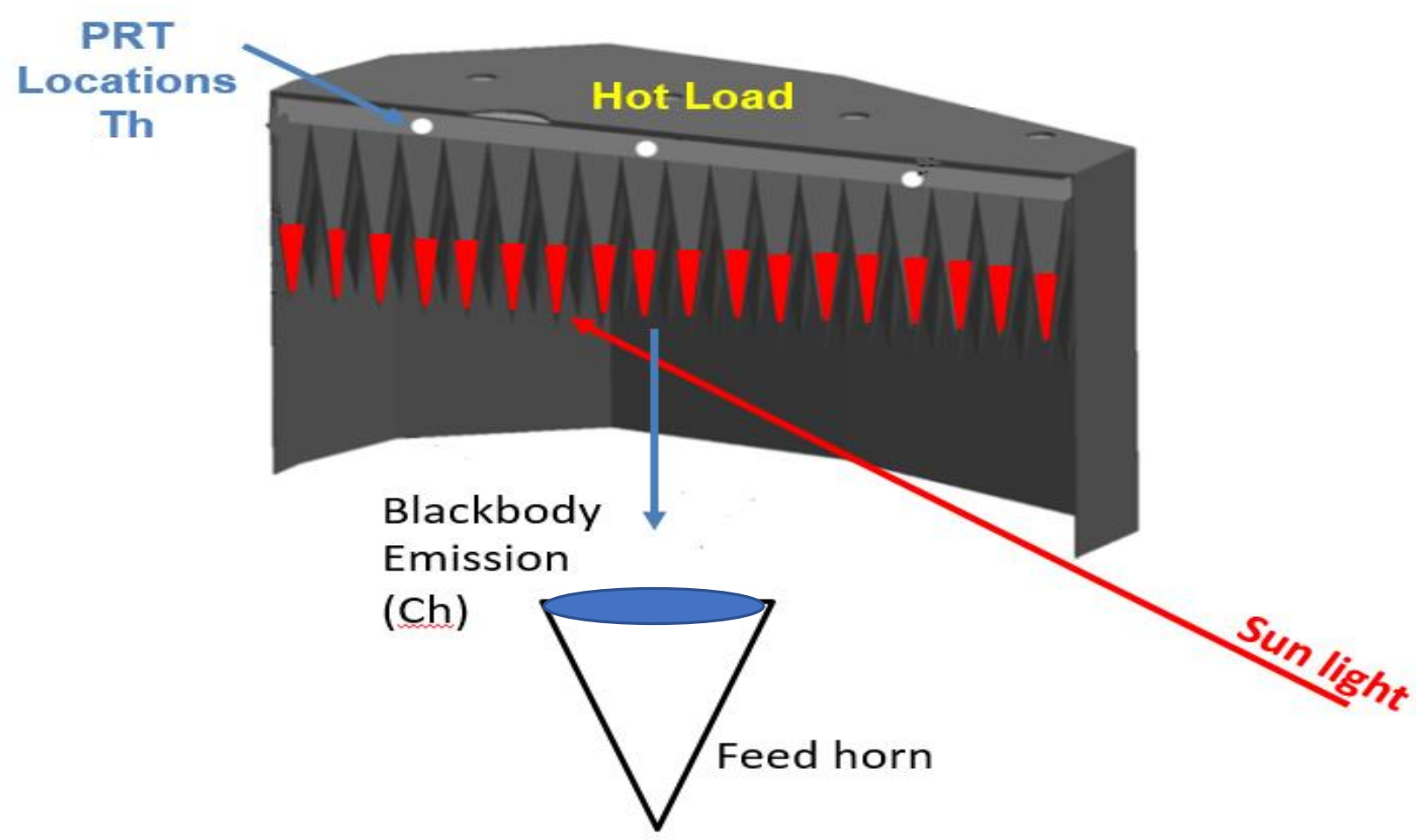

Figure 3.3: Sun intrusion heating the pyramidal tips of the hot load.

Before proceeding, it should be noted that a similar issue of the TMI measured hot load temperature is discussed by Wentz [2], and the author mitigated this by adjusting the hot load temperature to match the observed earth scene brightness temperature with theoretical brightness temperatures generated using the Remote Sensing Systems' Radiative Transfer Model temperature (RSS RTM). Thus, this is an empirical fix, which causes the hot load correction to match a specific radiative transfer model. However, in this dissertation, the hot load correction was based upon a rigorous analysis of the instrument transfer function, as it's described below. 


\subsection{Solar Coordinates Relative to TRMM Orbit Plane}

In this dissertation, the solar coordinates were based on the orbit plane since TRMM was flying with stable orientation. In Figure 3.4, the solar coordinates relative to TRMM's orbit are illustrated. The sun vector is called the sun line-of-sight which points directly to the origin of the TRMM coordinate system.

The first coordinate angle of the sun is represented by the solar beta angle $(\beta)$, which is the angle between the orbit plane and the solar line of sight; thus, this angle lies in the $-\mathrm{Z}$ and $-\mathrm{Y}$ TRMM plane. The zero beta angle occurs when the sun line-of-sight lies in the orbit pane, which corresponds to TRMM $-Z$ vector. Moreover, over seasons, TRMM on-orbit beta angle range is \pm 60 degree. The solar pointing requirement was to avoid the sun line-of-sight on the $+Y$ side of TRMM because of the cold sky reflector and the VIRS cooler. Therefore, TRMM alternately performed a yaw flip of 180 degrees, when the sun beta angle became negative and stayed at yaw 0 , when the sun beta angle was positive.

Next, the phase from orbit midnight is the second coordinate of the sun relative to TRMM's orbit. The angle of the solar line-of-sight was rotating clockwise in the $\mathrm{X}, \mathrm{Z}$ plane with the zero angle aligned at $-\mathrm{X}$ direction. The phase from orbit midnight completed one rotation every orbit. Thus, the positive phase angles correspond to eclipse time, while the negative angles are daylight. The specific phase angles for eclipse and daylight are specified by the solar beta angle. 


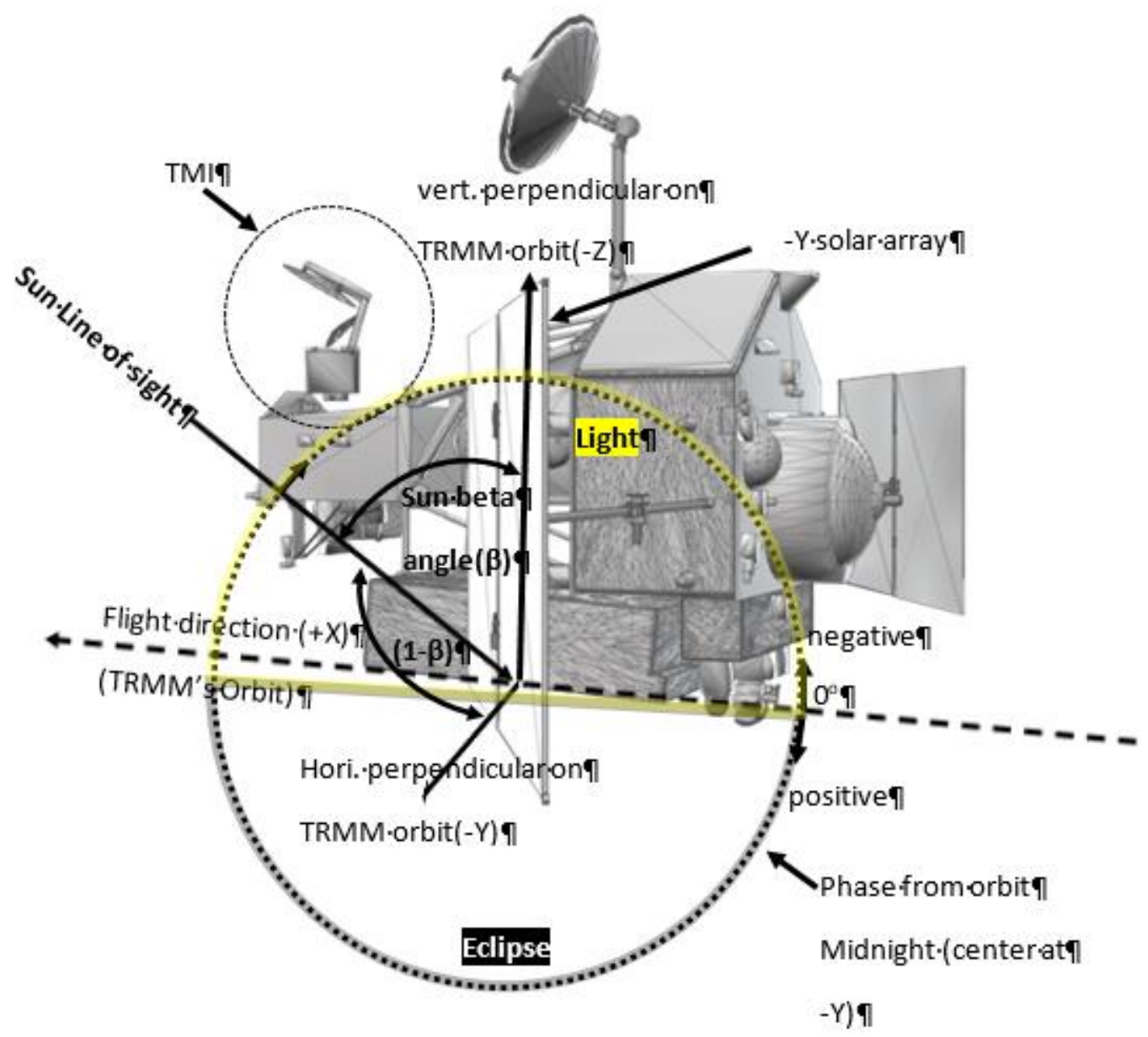

Figure 3.4: Solar coordinates relative to TRMM (TRMM model source: [4]).

\subsection{The Status of the -Y Solar Array}

The solar array rotation was controlled by the Solar Array Drive Actuators (SADA) to track the sun. At the beginning of launch, the array exceeded the energy requirement; so, for reliability purposes, it was decided to change the track angle from \pm 130 degrees to \pm 50 degrees. Later in the mission (2002), the SADA developed a problem, so after several months of testing, the team decided to feathered the solar array at zero degree, which is the X, Y plane [5]. Because the solar 
array position affected the solar intrusion of the hot load, it is documented in Appendix C, and Table 3.1 summarized all SADA tracking angles and corresponding dates of operation.

Table 3.1: The solar array action with the starting date.

\begin{tabular}{|l|l|l|l|}
\hline Period & Action & Start date & Duration \\
\hline 1 & Solar array tracking $\pm 130^{\circ}$ & $12 / 08 / 1997$ & 1 year \\
\hline 2 & $\begin{array}{l}\text { Solar array tracking } \pm 50^{\circ} \\
\text { Orbit Boost \& solar array is }\end{array}$ & $12 / 15 / 1998$ & $08 / 15 / 2001$ to $9 / 5 / 2002$, years \\
\hline 4 & $\begin{array}{l}\text { Orbit Boost } \& \text { feathered } \\
\text { a) test periods } \pm 1^{\circ}\end{array}$ & a) Feathered tests with $+/-1$-degree & $\sim 1$ year \\
b) parked $-Y$ array at $0^{\circ}$ & tracking in 2002 start dates $5 / 30,6 / 5$, & 13 years \\
& $\begin{array}{l}\text { 6/11, 6/24-8/12, } 9 / 5 \\
\text { b) } 10 / 11 / 2002 \text { parked at } 0 \text { (about orbit }\end{array}$ & \\
\hline
\end{tabular}

Because the rotating $-\mathrm{Y}$ solar array occasionally blocked the solar line of sight, the hot load correction depends on the solar array status. Thus, the analysis will be independently applied in each yaw orientation and time period. The exact orbits number for each period and yaw orientation are specified in Table 3.2. 
Table 3.2: Orbits range for each orientation and period.

\begin{tabular}{|l|l|l|}
\hline Period & \multicolumn{2}{|c|}{ Orbit \# range } \\
\hline Orientation & \multicolumn{1}{|c|}{ Yaw0 } & \multicolumn{1}{|c|}{ Yaw 180 } \\
\hline 1 & $160: 6026$ & $6027: 21400$ \\
\hline 2 & $6027: 21400$ & $21401: 27414$ (exceptions \\
\hline 3 & $\begin{array}{l}21401: 27414 \text { (exceptions } \\
\text { below) }\end{array}$ & below) \\
\hline 4 & $25884: 25885 *$ & $26069: 26072 *$ \\
& $25977: 25978 *$ & $27415: 27970 *$ \\
& $26273: 26664 *$ & $27971: 99100 * *$ \\
& $27038: 27393 *$ & \\
& $27415: 27970 *$ & \\
& $27971: 99100 * *$ & \\
\hline
\end{tabular}

*: testing for feathered array at \pm 1 -degree

**: solar array is feathered at 0 -degree

\section{$\underline{3.5 \text { Approach to Correct the Hot Load Brightness Temperature }}$}

As noted in Section 3.1, Fig. 3.1(d), sometimes the time series of the receiver gain did not change smoothly during an orbit cycle as was expected. Thus, we performed a polynomial regression to remove the transient fluctuation in the gain during the orbit phase between $-100^{\circ}$ to $-50^{\circ}$. This procedure was performed in three iterative steps, as illustrated in Figure 3.5. First, a regression fit was applied using the original data; next, the time series was edited to remove points 
with high negative residual (between the original data and the fit); and finally, the polynomial fit was repeated on these edited data to produce the smoothed estimated gain $\left(G_{f i t}\right)$.

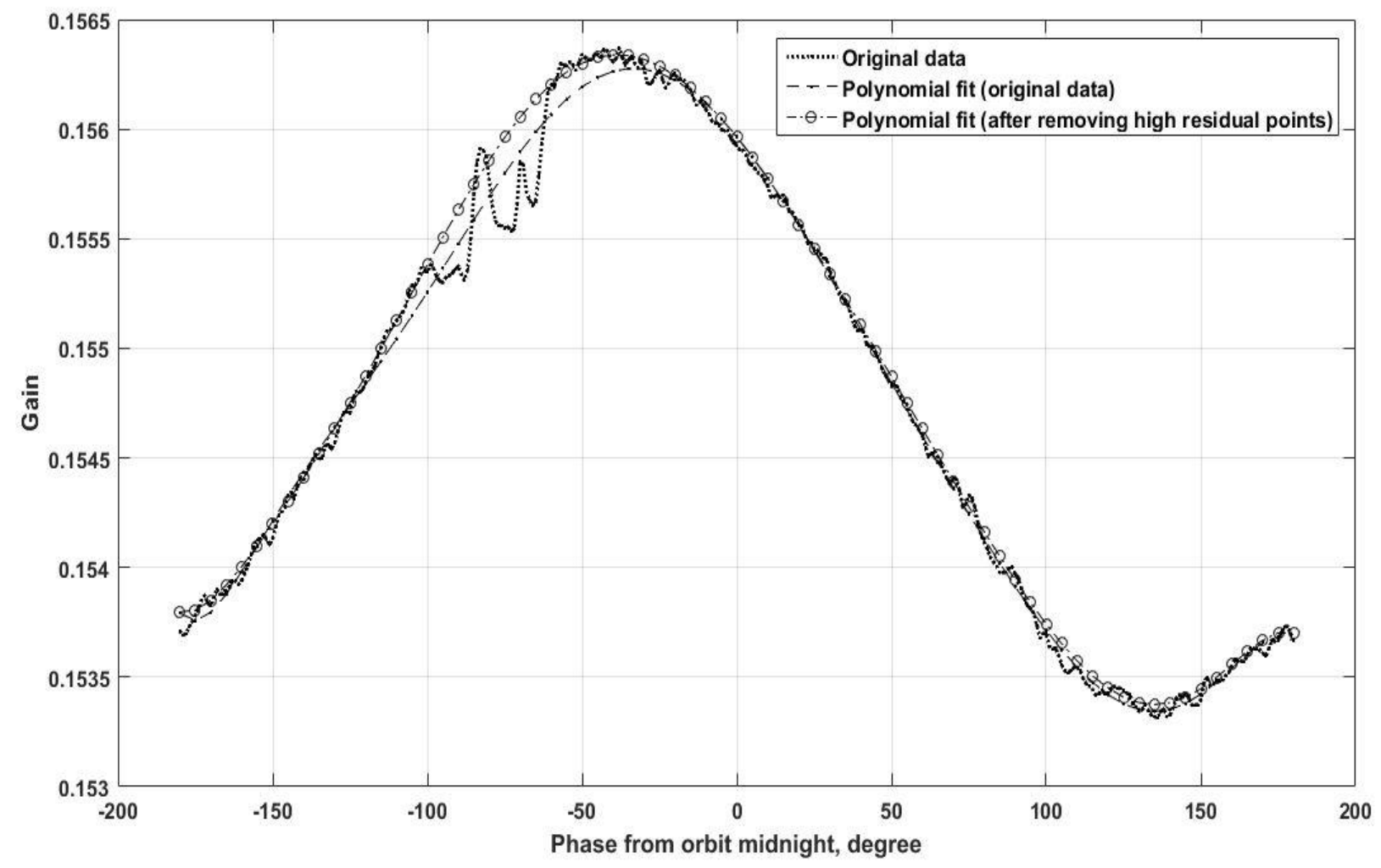

Figure 3.5: Polynomial fit for the radiometer gain time series to produce the hot load correction.

Finally, the correction of the hot load temperature $\left(\Delta T_{h}\right)$ was calculated as the difference between the corrected (smoothed) and the original hot load temperature as seen in Equation (3.4).

$$
\begin{gathered}
T_{h_{-} \text {cor }}=G_{f i t} *\left(C_{h}-C_{c}\right)+T_{c} \\
\Delta T_{h}=T_{h \_c o r}-T_{h}
\end{gathered}
$$

Since the geometry for the solar intrusion was repeatable for certain solar coordinates, this procedure was then applied to all orbits of each period (Table 3-2), and the resulting $\Delta T_{h}$ were bin 
averaged and filtered to form a hot load correction table for $> \pm 1$ Kelvin, over solar beta angle and orbit phase. It is important to note that while these corrections are empirically derived, they are independent of any RTM or inter-comparisons with other satellite radiometers.

\subsubsection{Unstable Receiver Gain}

As a part of the quality control effort to define the hot load correction, a careful examination was performed of the receiver orbital gain patterns for all channels over a range of beta angles for both yaws. Fortunately, after the hot load correction, all channels exhibited stable quasi-sinusoidal orbital receiver gain cycles, except for channel $21.3 \mathrm{~V}$ and channel $85.5 \mathrm{~V}$. These two channels have exhibited random patterns of significant "gain jumps", as seen in Figure 3.6. The reason for these unpredictable patterns is postulated to be temperature instability of the front-end low noise amplifiers of the receiver. Fortunately, the two-point radiometric calibration mitigated this effect and there was no apparent impact on the calibration accuracy.

During the low solar beta angles $(<10$ degrees), the gain of channels $21.3 \mathrm{~V}$ and $85.5 \mathrm{~V}$ returned to normal values, as shown in Figure 3.7; however, at high solar beta angles, the system exhibited large transient gain jumps. 

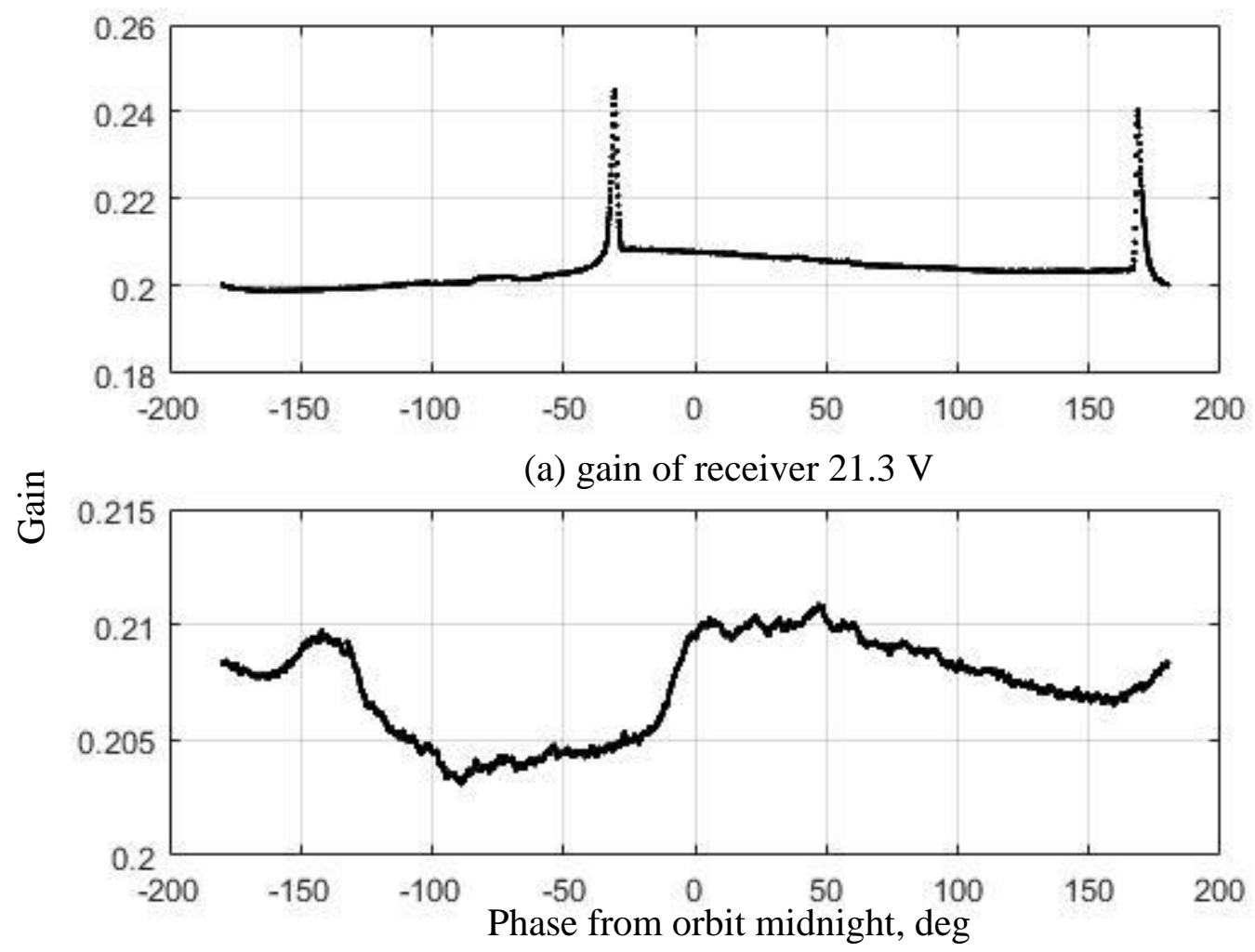

(b) gain of receiver $85.5 \mathrm{~V}$

Figure 3.6: Receiver gain of channel $21.3 \mathrm{~V}$ and $85.5 \mathrm{~V}$ at solar beta angle $=51.3^{\circ}$. 

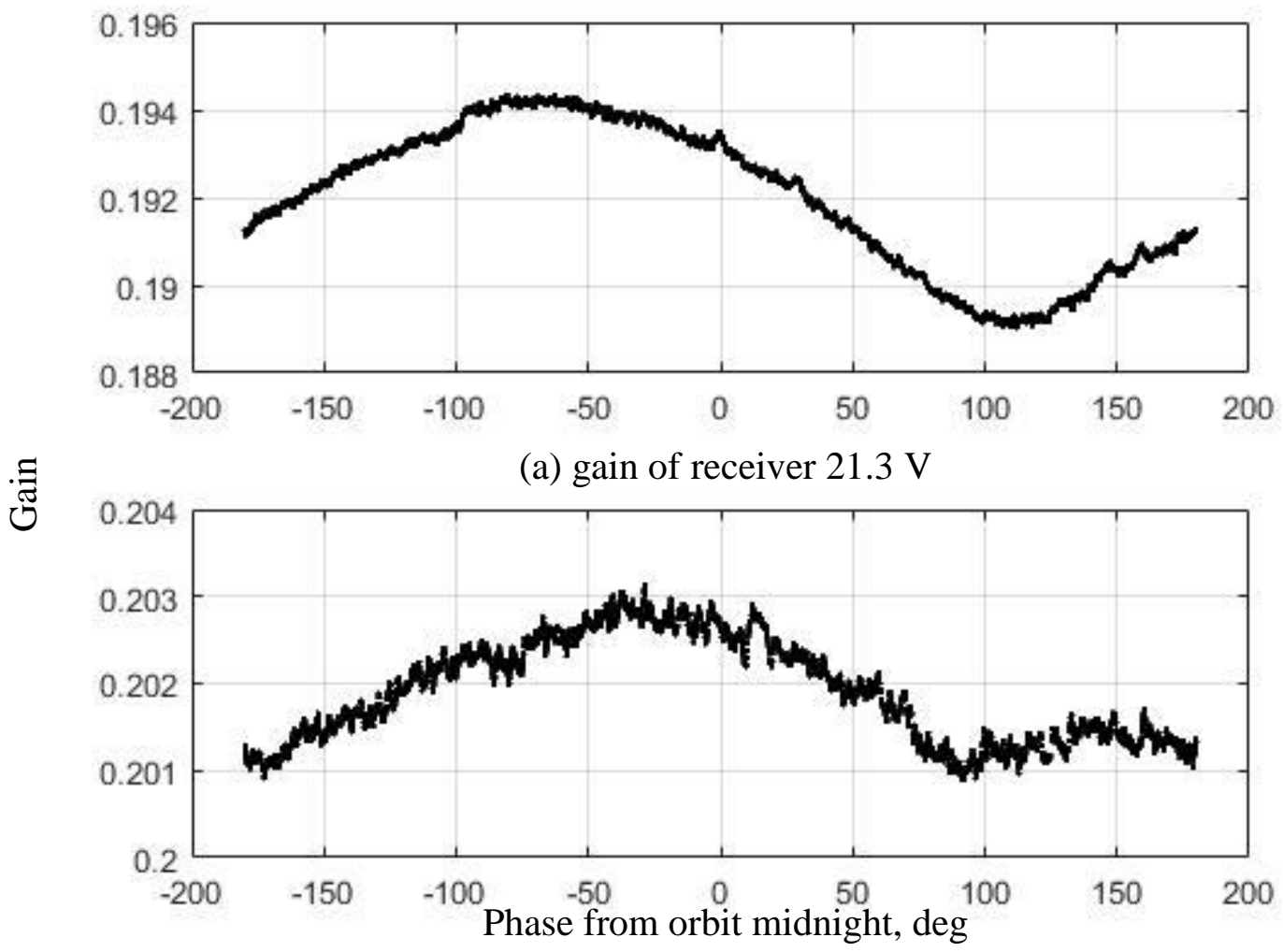

(b) gain of receiver $85.5 \mathrm{~V}$

Figure 3.7: Receiver gain of channel $21.3 \mathrm{~V}$ and $85.5 \mathrm{~V}$ at solar beta angle $=5.5^{\circ}$.

\section{$\underline{3.6 \text { Results }}$}

The hot load issue was caused by the direct impingement of solar flux on the blackbody target. Thus, the hot load correction is correlated with the sun coordinate relative to TRMM. The sun coordinates were expressed as the solar beta angle and the phase from orbit midnight relative to the TRMM orbit. Also, TRMM performs two different orientations: yaw 0 and 180 degrees relative to the flight direction that led to different results for each. Moreover, the solar tracking configuration of the $-\mathrm{Y}$ solar array was changing, which cause different results for each period (Table 3.1). 
It is worth mentioning that the gain of channels 5 and 8 are not stable and exhibit large gain jumps; however, the orbital receiver gain patterns of the other channels show very consistent results. For that reason, the hot load correction tables for channels 5 and 8 are estimated using linear interpolation of the neighbor channels.

In Fig. 3.8, the image of the hot load correction table is shown for the $10.65 \mathrm{~V}$ channel, when TRMM is at yaw 0-degree orientation and the -Y solar array was in period-1. The resolution of the orbit phase and the solar beta angle are $1^{\circ}$ and $0.25^{\circ}$, respectively. The majority of the hot load correction was between phase $-100^{\circ}$ and $-75^{\circ}$, especially at high sun beta angles. This was expected since the solar line of sight was closer to the exposed hot load pyramids, as seen in Figs. 3.2 and 3.4. Although the sun line-of-sight was still toward the hot load after the $-75^{\circ}$ orbit phase, the - Y solar array blocked the light because the solar array's tracking angle of period- 1 was $\pm 130^{\circ}$. Finally, for convenience, the hot load correction table was used to correct a small cold load issue. A small error was introduced in the cold counts (see Fig. 3.9), when the rotating -Y solar array at high tracking angles intersected the cold sky mirror IFOV, as shown in Fig. 3.10 [6]. This effect raised the cold load counts; so the equivalent effect was produced by lowering the hot load brightness temperature by $0.1 \mathrm{~K}$ between the orbit phase from $65^{\circ}$ to $75^{\circ}$. 


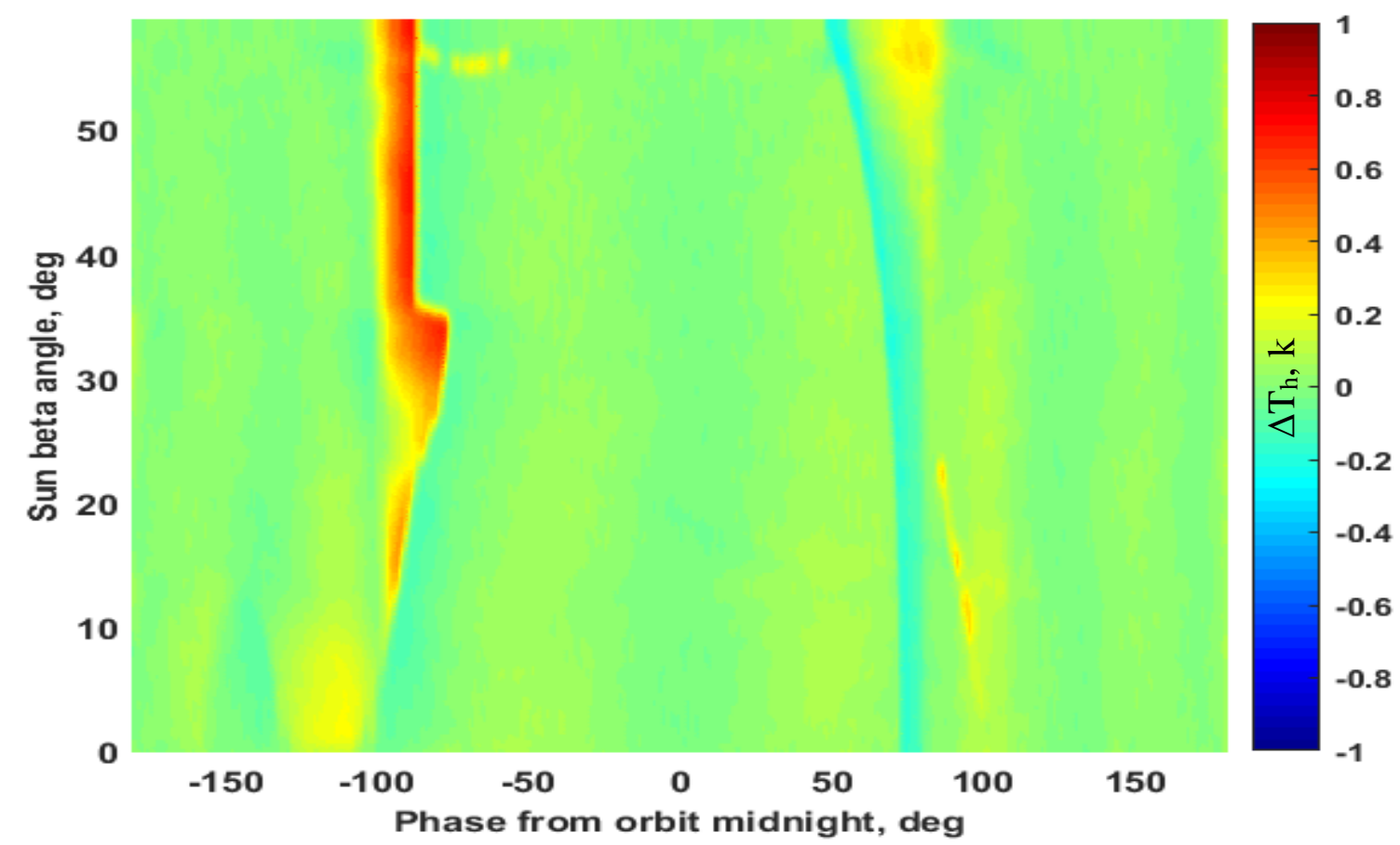

Figure 3.8: Hot load correction table for channel $10.65 \mathrm{~V}$, yaw0, and period-1.

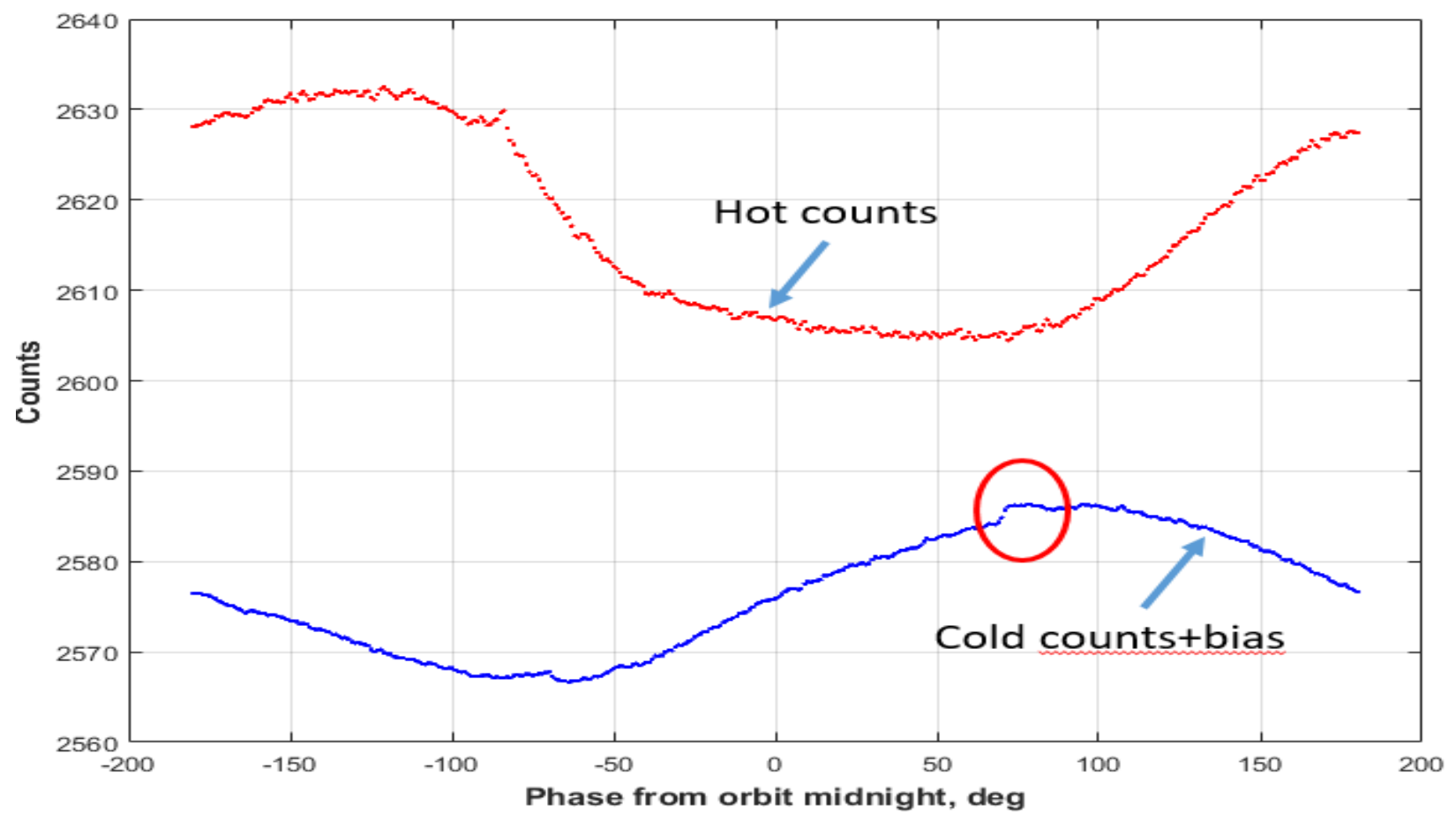

Figure 3.9: The effect of the intersection between the cold sky reflector beam and the -Y solar array in the cold counts measurements. 


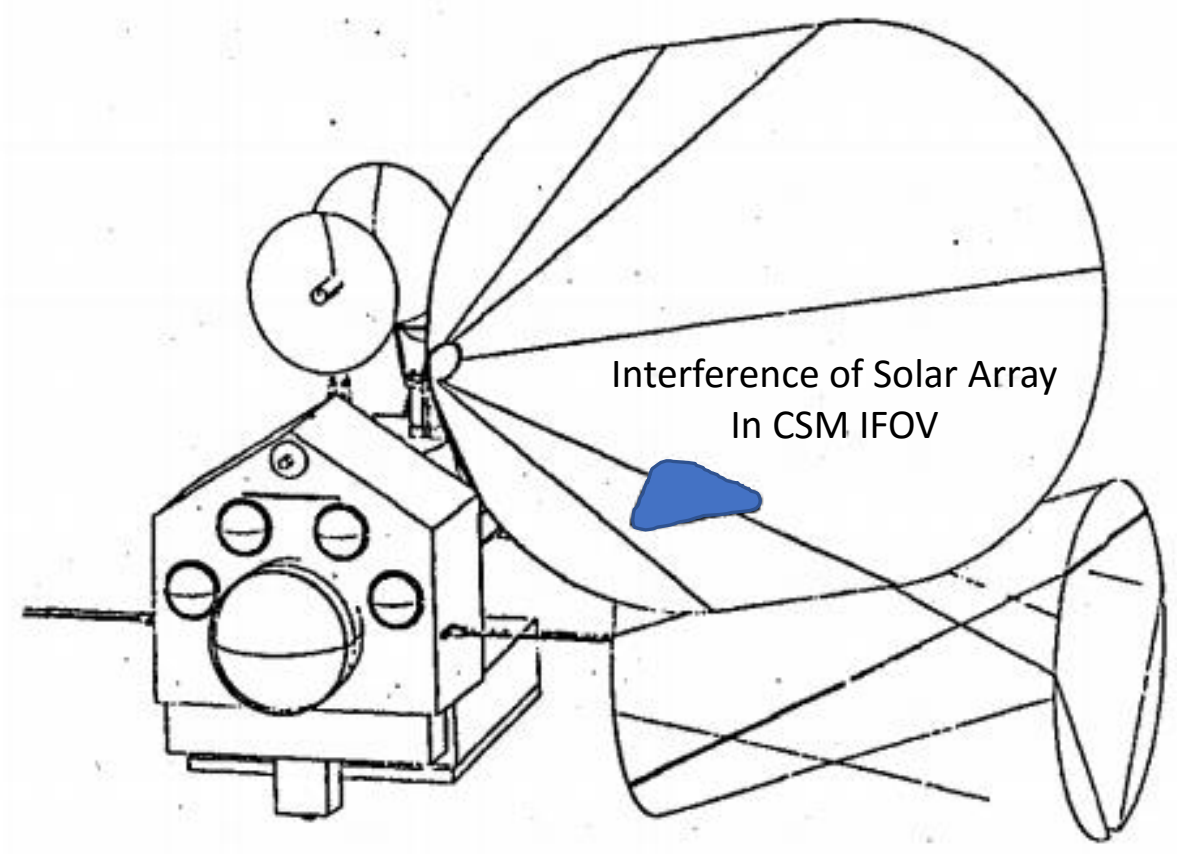

Figure 3.10: The intersection between the cold sky reflector beam and the -Y solar array at high tracking angles (Source: [6]).

During period-2, the tracking angle of the $-\mathrm{Y}$ solar array was changed to be $\pm 50^{\circ}$. Thus, the sun light was passed to the hot load correction in the phase angle around $-60^{\circ}$, as shown in Fig. 3.11. Next, TRMM was boosted to a higher altitude during period-3, which caused the sun intrusion to the hot load to be over a wider range at phase $-60^{\circ}$, as shown in Fig. 3.12. Finally, during period-4, the solar array was feathered at a horizontal level (zero-degree), which resulted in a wider range of phase angles for the sun intrusion, as shown in Figure 3.13. The results for all of the other channels, periods, and orientations show consistent results for each feed horn (see Appendix D). 
All of these results were used to correct the measured hot load temperature in Equations (2.1) and (2.2), and the corrected gain $\left(G_{c o r}\right)$ and corrected offset $\left(\right.$ Offset $\left.t_{c o r}\right)$ were calculated by using the hot load temperature correction given in the following equations:

$$
\begin{gathered}
G_{c o r}=\frac{\left(T_{h}+\Delta T_{h}\right)-T_{c}}{C_{h}-C_{c}} \\
\text { offset }_{c o r}=G_{c o r} * C_{c}-T_{c}
\end{gathered}
$$

Because the hot load correction is a multiplicative term, as seen in Equation (2.3), it will change the antenna temperature depending upon both antenna temperature and the corresponding hot load correction. For example, the maximum hot load correction in channel $1(10.65 \mathrm{~V})$ is around $0.9 \mathrm{~K}$, and assuming different values of constant hot load correction in TMI's orbit 87736, yields to the results presented in Fig. 3.14. The greatest change occurs at the highest antenna temperature which is close to the hot load temperature, while the minimum change occurs at the lowest antenna temperature at $165 \mathrm{~K}$ for channel $10.65 \mathrm{~V}$ ( 55\% of the hot load correction value). The weight of the hot load correction depends on the ratio of the hot load temperature to the antenna temperature, as:

$$
\Delta T_{a}=\frac{T_{a}}{T_{h}} * \Delta T_{h}
$$




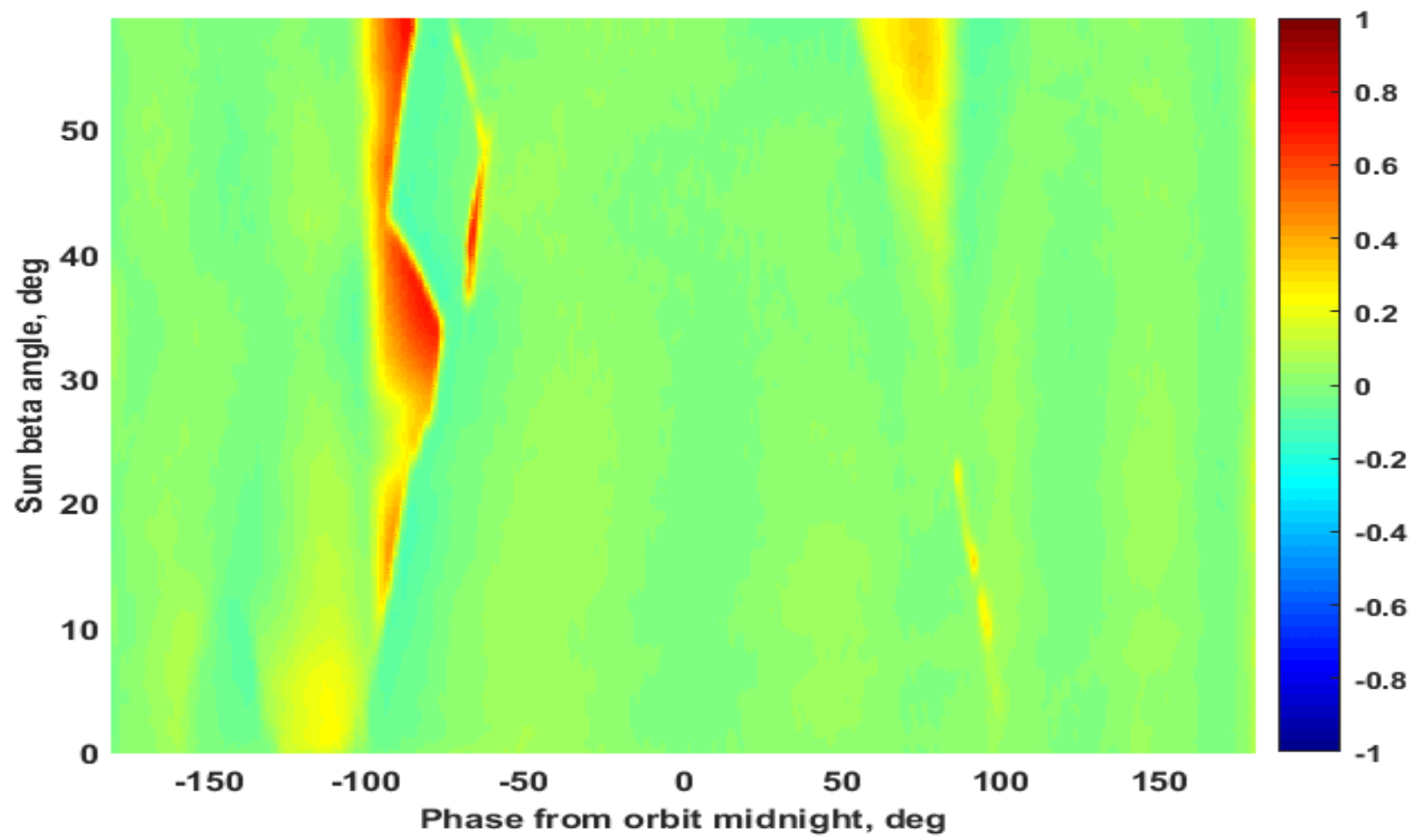

Figure 3.11: Hot load correction table for channel $10.65 \mathrm{~V}$, yaw0, and period 2.

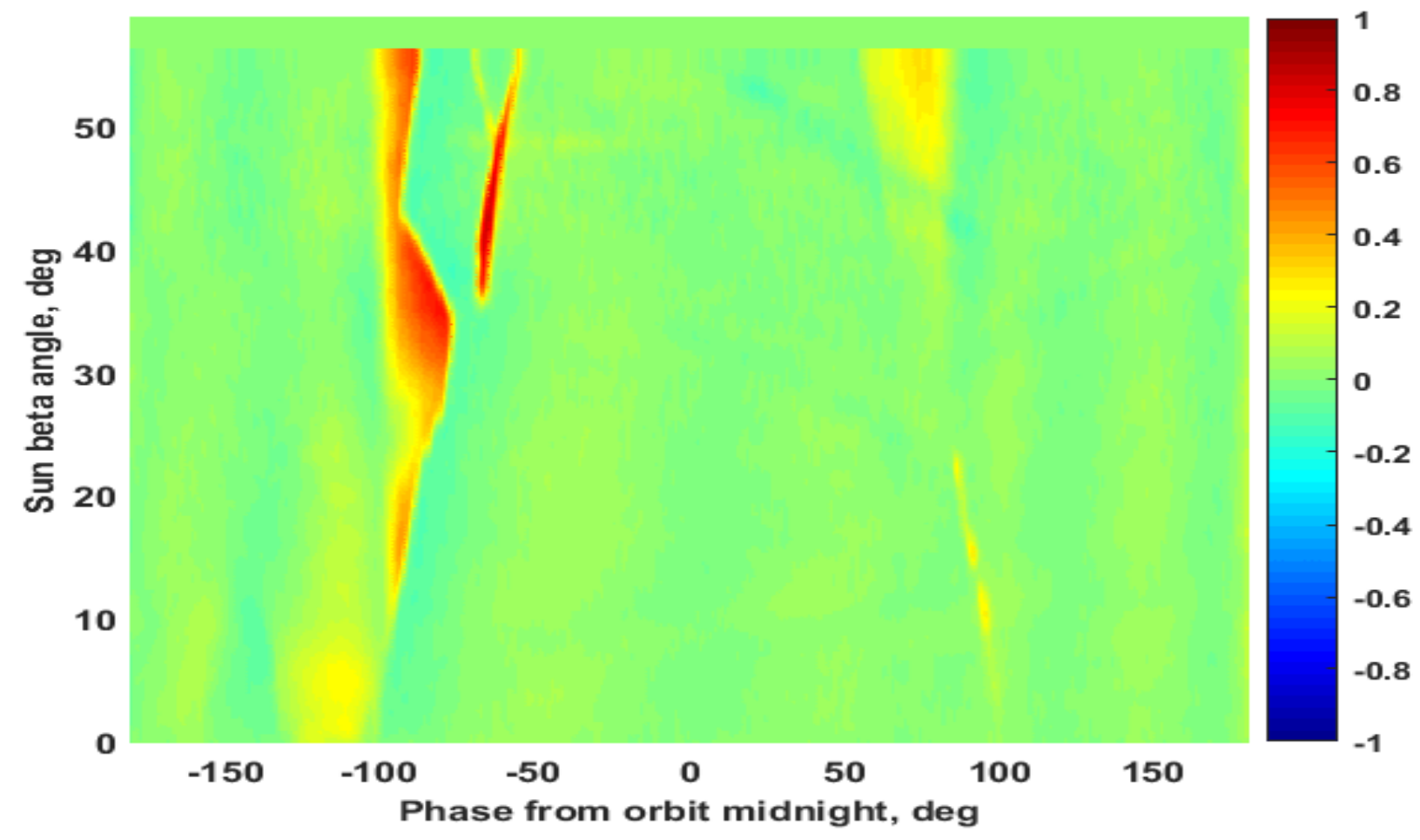

Figure 3.12: Hot load correction table for channel $10.65 \mathrm{~V}$, yaw0, and period 3. 


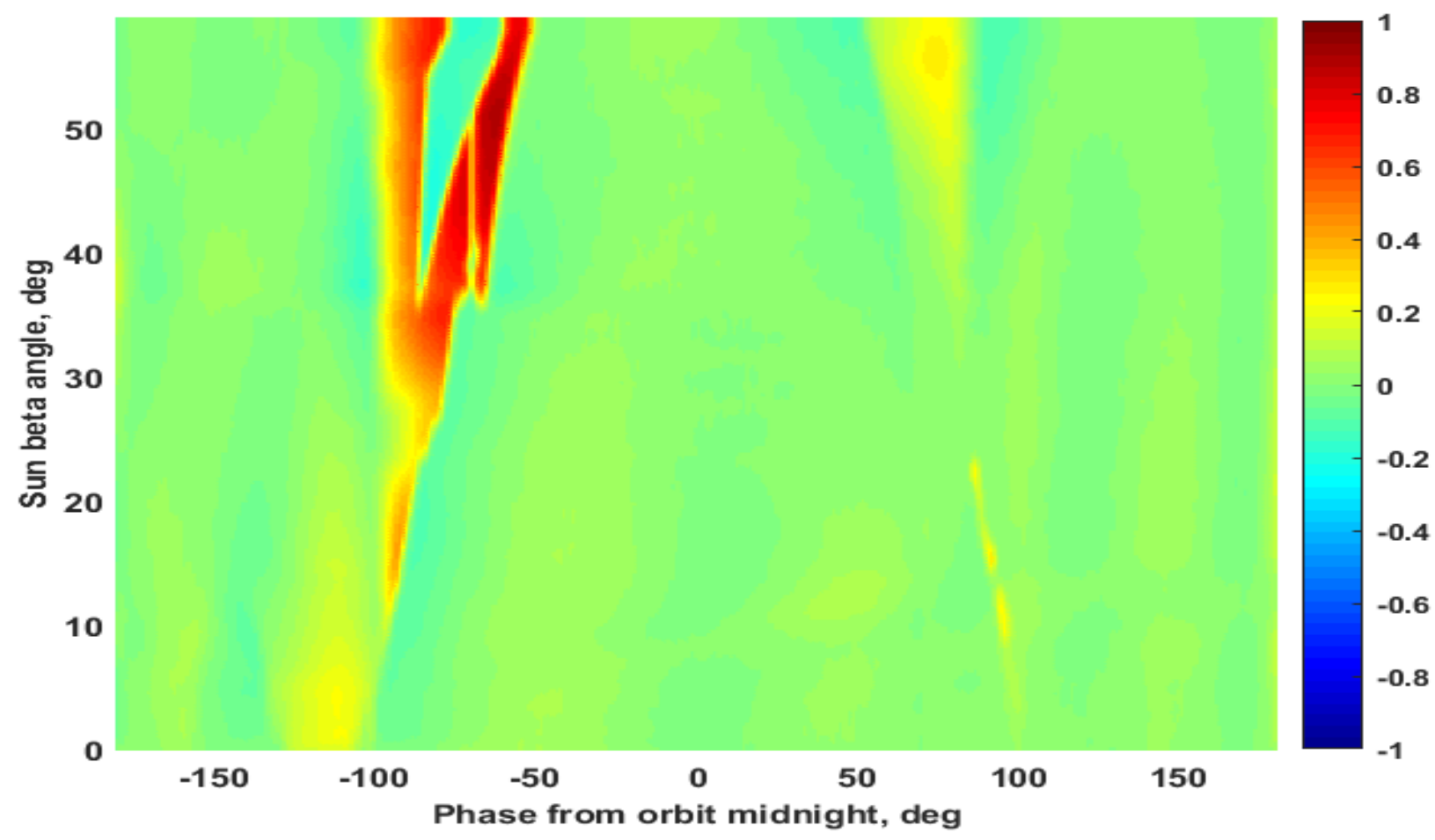

Figure 3.13: Hot load correction table for channel $10.65 \mathrm{~V}$, yaw0, and period 4.

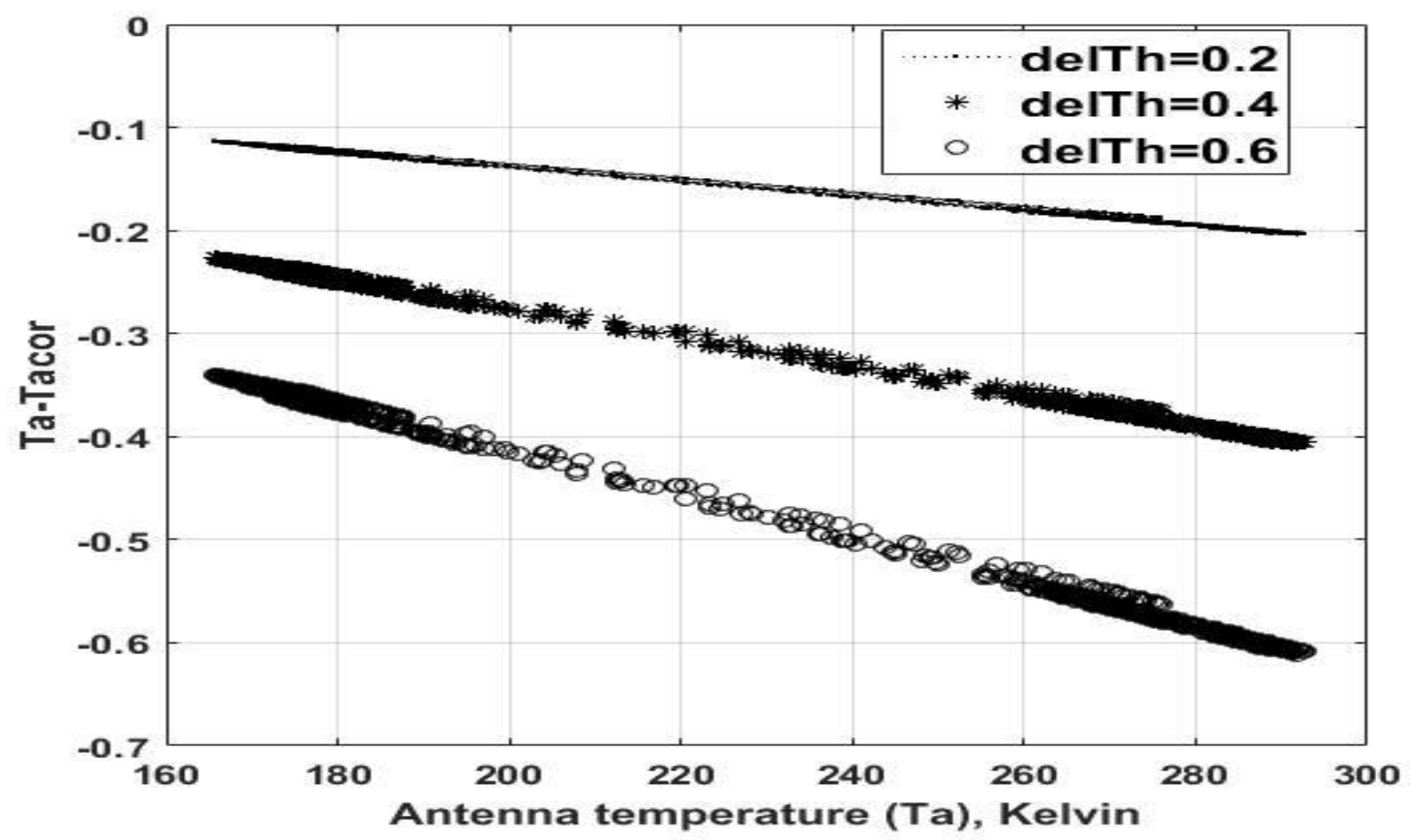

Figure 3.14: The hot load correction effect on the antenna temperature. 


\section{$\underline{3.7 \text { References }}$}

[1] Z. Ghazi, "CONAE microwave radiometer (MWR) counts to brightness temperature algorithm," Ph.D. dissertation, Dept. Electrical and Computer Engineering, University of Central Florida, Orlando, FL, 2014.

[2] F. J. Wentz, "A 17-yr climate record of environmental parameters derived from the Tropical Rainfall Measuring Mission (TRMM) Microwave Imager," Journal of Climate, vol. 28 , no. 17, pp. 6882-6902, 2015.

[3] J. Shiue, personal communication, Unpublished.

[4] TRMM model from NASA website available at: https://data.nasa.gov/SpaceScience/NASA-3D-Models-TRMM/357b-ra7j/data ,2017.

[5] T. Nio, S. Saito, E. Stocker, J. H. Pawloski, Y. Murayama, and T. Ohata, "Tropical Rainfall Measurement Mission (TRMM) Operation Summary," 2015.

[6] "Tropical Rainfall Measuring Mission Microwave Imager (TMI): Critical Design Review," Hughes, Sept 1993, unpublished. 


\section{CHAPTER 4: EMISSIVE ANTENNA CORRECTION}

F. Alquaied, W. Jones, “An emissive antenna correction for the Tropical Rainfall Measuring Mission Microwave Imager (TMI)", Proc. IEEE International Geoscience and Remote Sensing (IGARSS), Fort Worth, USA, July 23-28, 2017, accepted for publication.

This chapter presents a discussion of the development of the algorithm for making an emissive brightness correction for TMI, which will be included in the legacy version (V8) of the 1B11 brightness temperature product. This chapter is the major focus of this dissertation.

During TMI prelaunch radiometric calibration, a comprehensive thermal vacuum test was performed that simulated the on-orbit environment; but during this test, only the antenna feed was used (i.e., the parabolic reflector was not part of the calibration process). Therefore, the first time that the radiometer "viewed the reflector" was on-orbit.

An ideal metal parabolic mirror (reflector) provides a perfect reflection of the earth scene brightness temperature into the receiver; however, an imperfect (slightly emissivity) reflector partially reflects the earth scene and emits an unwanted temperature bias that is added to the scene temperature as:

$$
\overline{T_{b, l}}=\left(1-\varepsilon_{i}\right) T_{b, i}+\left(\varepsilon_{i}\right) T_{p h y}
$$

where $(1-\varepsilon)$ is the parabolic mirror reflectivity, $\varepsilon$ is the reflector emissivity, $T_{p h y}$ is the reflector physical temperature, and the product $(\varepsilon) T_{p h y}$ is the reflector emission component of the brightness

temperature. After applying the hot load correction, $\overline{T_{b, l}}$ is calculated using Equation (2.12), and the desired scene brightness temperature $T_{b, i}$ can be redefined as: 


$$
T_{b, i}=\frac{\overline{T_{b, l}}-\left(\varepsilon_{i}\right) T_{p h y}}{\left(1-\varepsilon_{i}\right)}
$$

The construction of the TMI main reflector was a graphite-epoxy shell that was coated by a thin layer of vapor deposited aluminum (VDA), which was expected to provide a highly reflective surface. However, during on-orbit check-out, Wentz et al. [1] discovered that TMI earth scene Tb's were anomalously high. After an intensive investigation, they determined that the main reflector was slightly emissive; thus, channel emissivity values were derived during the postlaunch $\mathrm{Cal} / \mathrm{Val}$ period by comparing TMI's measurements with SSM/I measurements, and more recently the results were updated in [2].

As a part of this dissertation, the channel emissivity values were independently derived based on the analysis of a "Deep Space Maneuver" (DSM) that was performed in 2015. This more rigorous approach is based solely on radiometric measurements of the known cosmic microwave background (CMB), which avoids uncertainties associated with the use of an ocean RTM or intersatellite cross calibration to derive each TMI channel emissivity.

\subsection{Deep Space Maneuver (DSM)}

Space is an excellent radiometric calibration target because the cosmic microwave background (CMB) presents a known, stable, non-polarized, homogeneous and isotropic brightness temperature of 2.7 to 3.2 Kelvin over the TMI frequency range [3]. For the past two decades, space-borne microwave radiometers have relied upon DSMs, where satellites perform attitude maneuvers that cause the conical-scanning radiometer antenna main beam to view space for a significant portion (delta-Az) of the active scan. 
At the beginning and the end of the TRMM mission, there were a total of twenty DSMs performed to provide "end-to-end" radiometric calibration for TMI, and the details are summarized in Table 4.1 that include the date, TRMM orbit number, yaw orientation and altitude.

Table 4.1: Deep Space Maneuvers (DSMs) during TRMM mission.

\begin{tabular}{|c|c|c|c|c|}
\hline Maneuver \# & Date (mm/dd/yyyy) & Orbit \# & Orientation & Altitude (Km) \\
\hline 1 & $01 / 07 / 1998$ & 642 & 180 & 348.57 \\
\hline 2 & 01/07/1998 & 644 & 180 & 348.53 \\
\hline 3 & $01 / 07 / 1998$ & 646 & 180 & 348.53 \\
\hline 4 & 01/08/1998 & 658 & 180 & 348.36 \\
\hline 5 & 01/08/1998 & 660 & 180 & 348.31 \\
\hline 6 & 01/08/1998 & 662 & 180 & 348.34 \\
\hline 7 & 09/02/1998 & 4394 & 0 & 349.54 \\
\hline 8 & $07 / 22 / 2014$ & 95023 & 0 & 397.39 \\
\hline 9 & $07 / 22 / 2014$ & 95025 & 0 & 397.40 \\
\hline 10 & $07 / 22 / 2014$ & 95027 & 0 & 397.46 \\
\hline 11 & $02 / 26 / 2015$ & 98452 & 180 & 353.75 \\
\hline 12 & $02 / 26 / 2015$ & 98454 & 180 & 354.49 \\
\hline 13 & $02 / 26 / 2015$ & 98456 & 180 & 354.69 \\
\hline 14 & $02 / 27 / 2015$ & 98468 & 180 & 355.02 \\
\hline 15 & $02 / 27 / 2015$ & 98470 & 180 & 355.04 \\
\hline 16 & $02 / 27 / 2015$ & 98472 & 180 & 355.22 \\
\hline 17 & $03 / 25 / 2015$ & 98879 & 90 & 336.67 \\
\hline
\end{tabular}




\begin{tabular}{|c|c|c|c|c|}
\hline Maneuver \# & Date (mm/dd/yyyy) & Orbit \# & Orientation & Altitude (Km) \\
\hline $\mathbf{1 8}$ & $03 / 25 / 2015$ & 98882 & 90 & 336.76 \\
\hline $\mathbf{1 9}$ & $03 / 26 / 2015$ & 98894 & 90 & 336.41 \\
\hline $\mathbf{2 0}$ & $03 / 26 / 2015$ & 98897 & 90 & 336.18 \\
\hline
\end{tabular}

For both TRMM yaw orientations $\left(0^{\circ}\right.$ or $\left.180^{\circ}\right)$, the DSM were performed by entering into an "inertial hold mode" that caused the spacecraft to perform a $360^{\circ}$ rotation in the pitch axis over the orbit period, as illustrated in Fig. 4.1. Thus, concerning the TMI antenna pointing, there were 3 phases, designated as $\mathrm{A}, \mathrm{B}$ and $\mathrm{C}$, and associated with the antenna subsystem, there were three beams, namely: conical rotating main beam (MB - black arrow), the main reflector spill-over beam (SO - red arrow) and the fixed cold sky beam (CSB - green arrow).

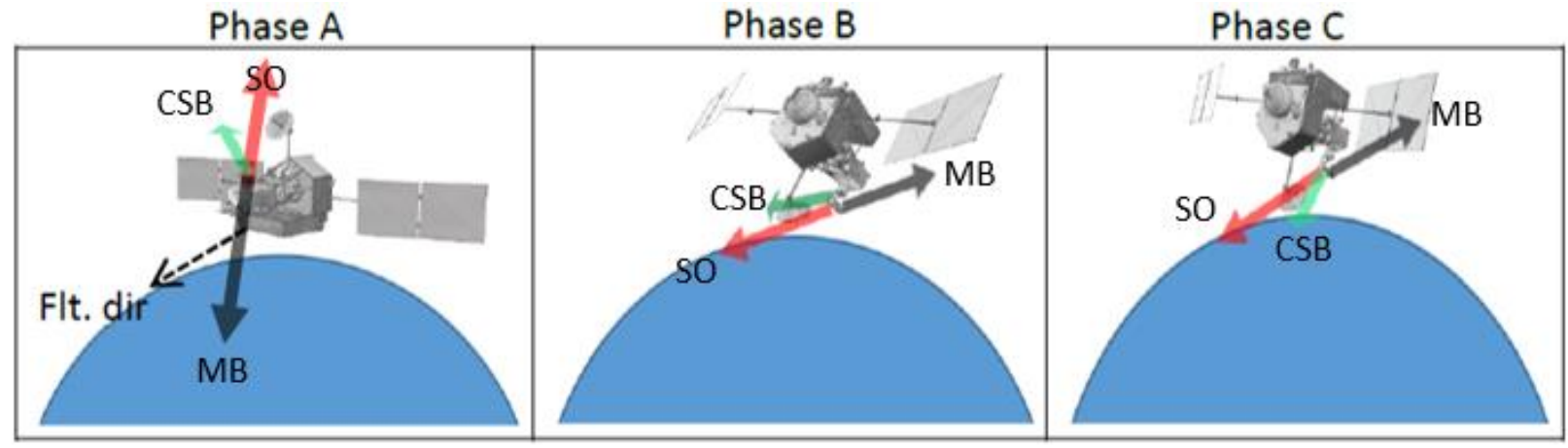

Figure 4.1: Example of a typical DSM showing TMI phases for the spacecraft yaw $=0$ degrees.

In phase-A (normal operation) the conical scanning main beam views earth, while the cold sky beam and the spill-over beam view space. After $>90^{\circ}$ change in pitch attitude, TMI enters phase- $\mathrm{B}$, where the MB views space, but the SO beam intersects with the earth. Thus, the total received brightness was the sum of cold space brightness (from the main beam) and warm 
brightness from earth (through the SO beam). This effect can be observed in the time series of antenna brightness temperature $\left(T_{A}\right)$ for DSM\#11 that is presented in Fig. 4.2. At this time, the SO beam views the earth near the nadir, and when the sub-track passes over the tip of Australia, the antenna brightness temperature increases by $\sim 0.5 \mathrm{~K}$.

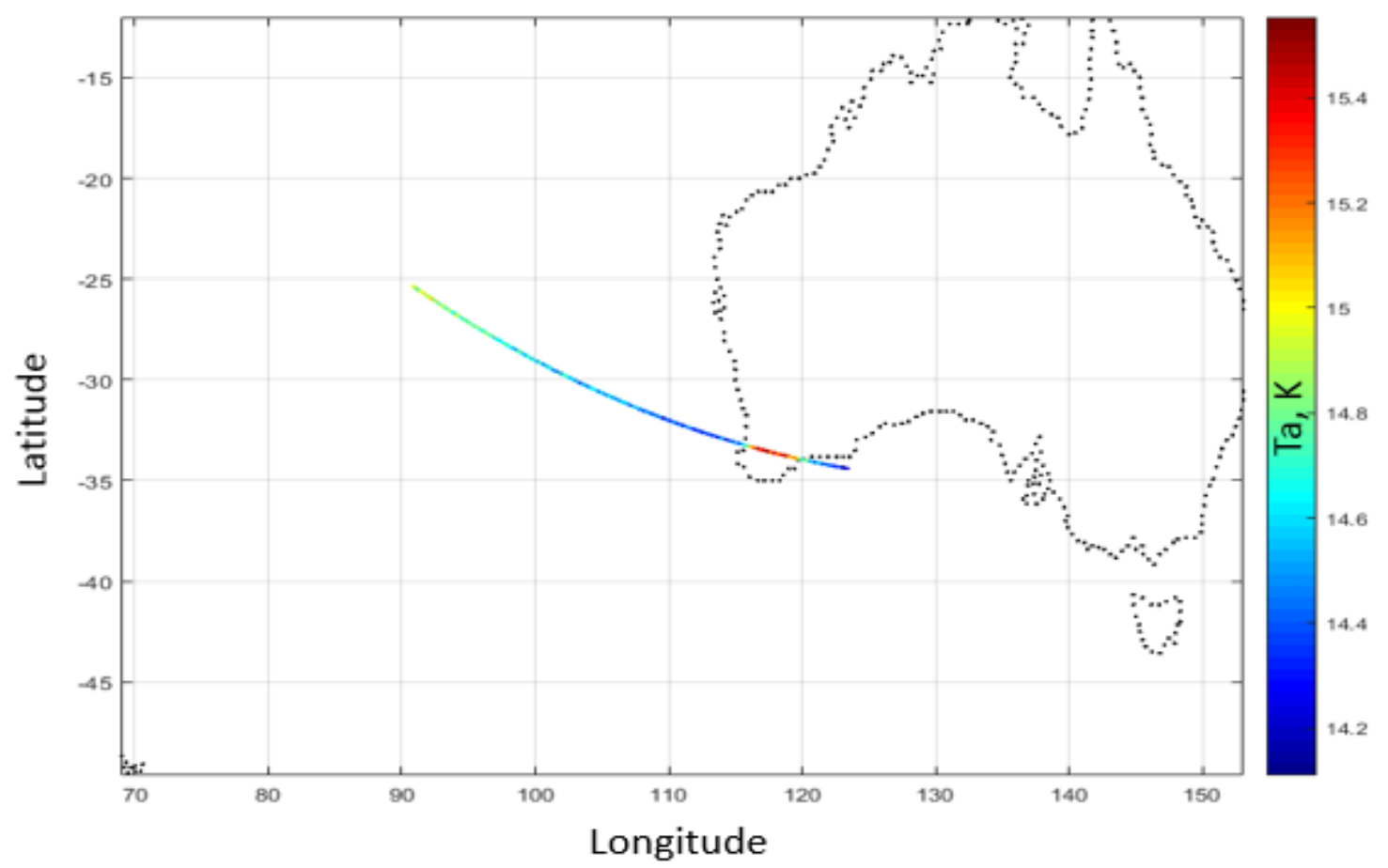

Figure 4.2: Measured antenna temperature (10.65V) shown as a colored trace along the satellite sub-track during the DSM\#11.

As the spacecraft continues the pitch rotation, TMI enters phase-C where the CSB intersects with the earth for the remaining time of the maneuver. At this time, the two-point radiometric calibration is corrupted because the CMB views the unknown earth brightness, which results in unrealistic gain and antenna temperatures. As noted, there is no time where all beams view space, which is required to characterize the main reflector emissivity. 
In 2015, just before the end of the mission, TRMM performed four DSMs where the spacecraft was rotated in yaw by $90^{\circ}$ before entering inertial mode. This resulted in TRMM being rotated $360^{\circ}$ about the roll axis over one orbit period, which modified TMI beams pointing to space, as described below.

During this "yaw-90 DSM", TMI antenna beams went through four different phases, as seen in Fig. 4.3. The first, phase-A, illustrates the spacecraft yawed by $90^{\circ}$, which caused the scanning $\mathrm{MB}$ to view the earth while the SO and the CSB were pointed to space. Next, the spacecraft rotation was started, and after the spacecraft is rolled by $\sim 75^{\circ}$, TMI enters phase-B, where the MB and SB pointed to space, but the CSB intersected the earth. After the spacecraft rolled by $\sim 200^{\circ}$, TMI entered phase-C, which caused both MB and CSB to point to space, but now the SO beam intersected the earth. Finally, phase-D occurred when the spacecraft rotation was $\sim 290^{\circ}$, and at that moment all beams were simultaneously pointed to space, which was used to derive the reflector emissivity at the various channel frequencies.

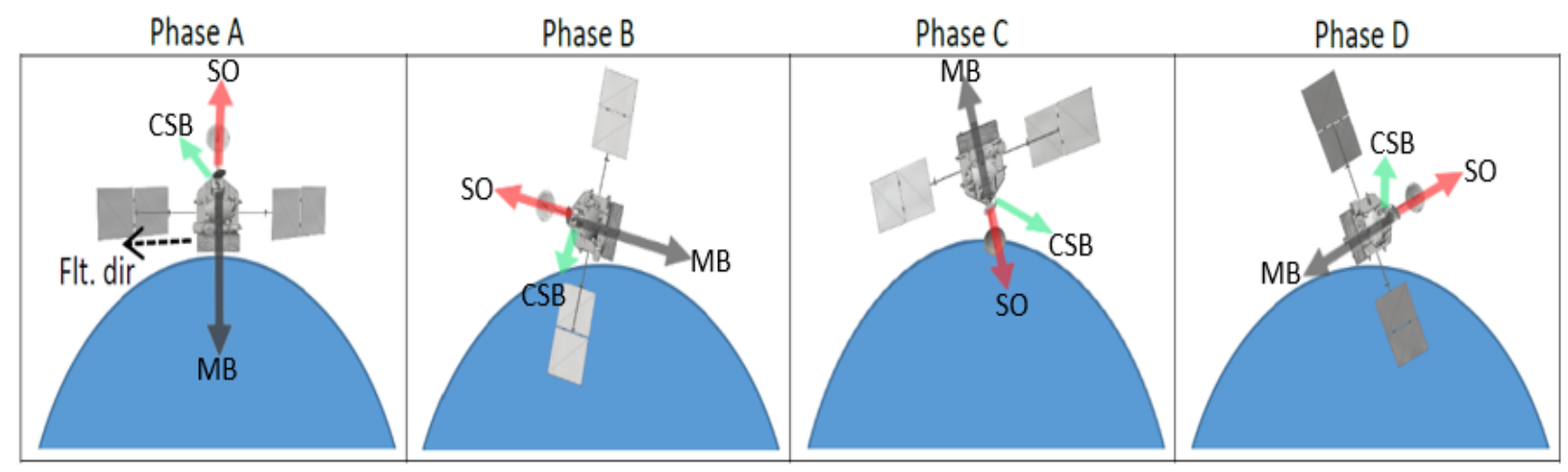

Figure 4.3: TRMM phases during the DSM at yaw 90 degrees.

The time series of antenna brightness temperature $\left(T_{A}\right)$, averaged over azimuth beam positions $(70-80)$, is shown in Fig. 4.4 for the four phases of the DSM. In phase-A, TMI is in the 
normal operation mode where the $T_{A}$ of channel $1(10.65 \mathrm{~V})$ ranges between land $(280 \mathrm{~K})$ and ocean $(180 \mathrm{~K})$. In the second phase-B, where the CSB pointed to the hot earth, the calibration procedure was corrupted resulting in negative $T_{A}$. In the third phase-C, the CSB departed the earth and pointed to space, so the calibration returned to normal, but now the SO beam intercepted the warm earth. As the spacecraft rotation continued, the SO beam eventually scanned off the earth; and at this point, the $T_{A}$ decreased with time until all three beams were pointed to space, when $T_{A}$ reached a minimum value, as shown in Figure 4.4(b). After several minutes ( 2900 scans), the MB returned to view the earth at the end of the DSM (not shown).

Since the SO beam intersected the earth during phase-C, the antenna temperature was increased over the CMB by the addition of hot land Tb coupled through the SO beam, as seen in Fig. 4.5. However, during the fourth phase-D, the measured $\mathrm{T}_{\mathrm{A}}$ was not affected by the earth scene. 

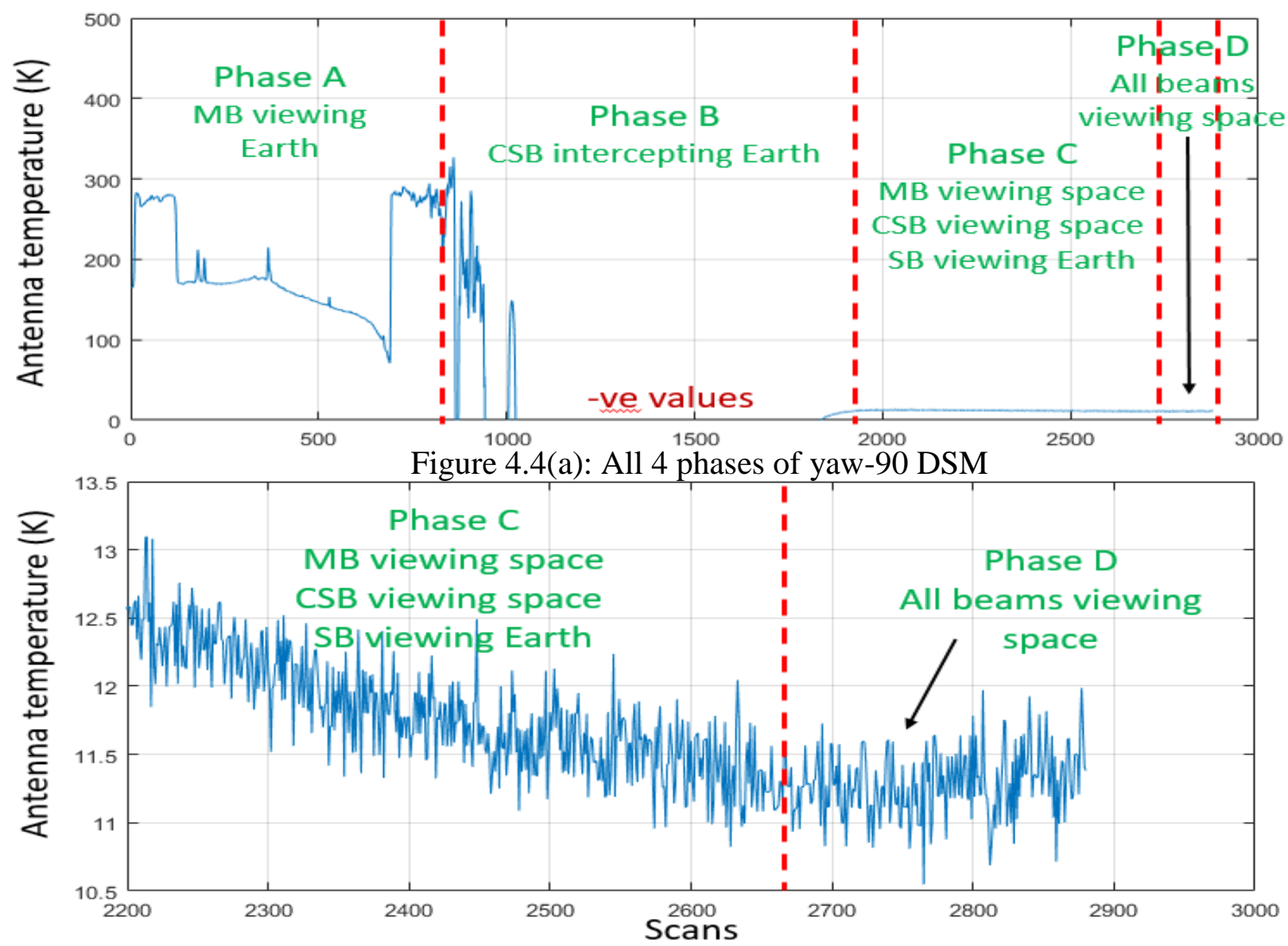

Figure 4.4(b): Last two phases of yaw-90 DSM

Figure 4.4: Antenna brightness temperature (10.65 V) during 4 phases of the DSM\#18, performed at a spacecraft yaw of $90^{\circ}$. 


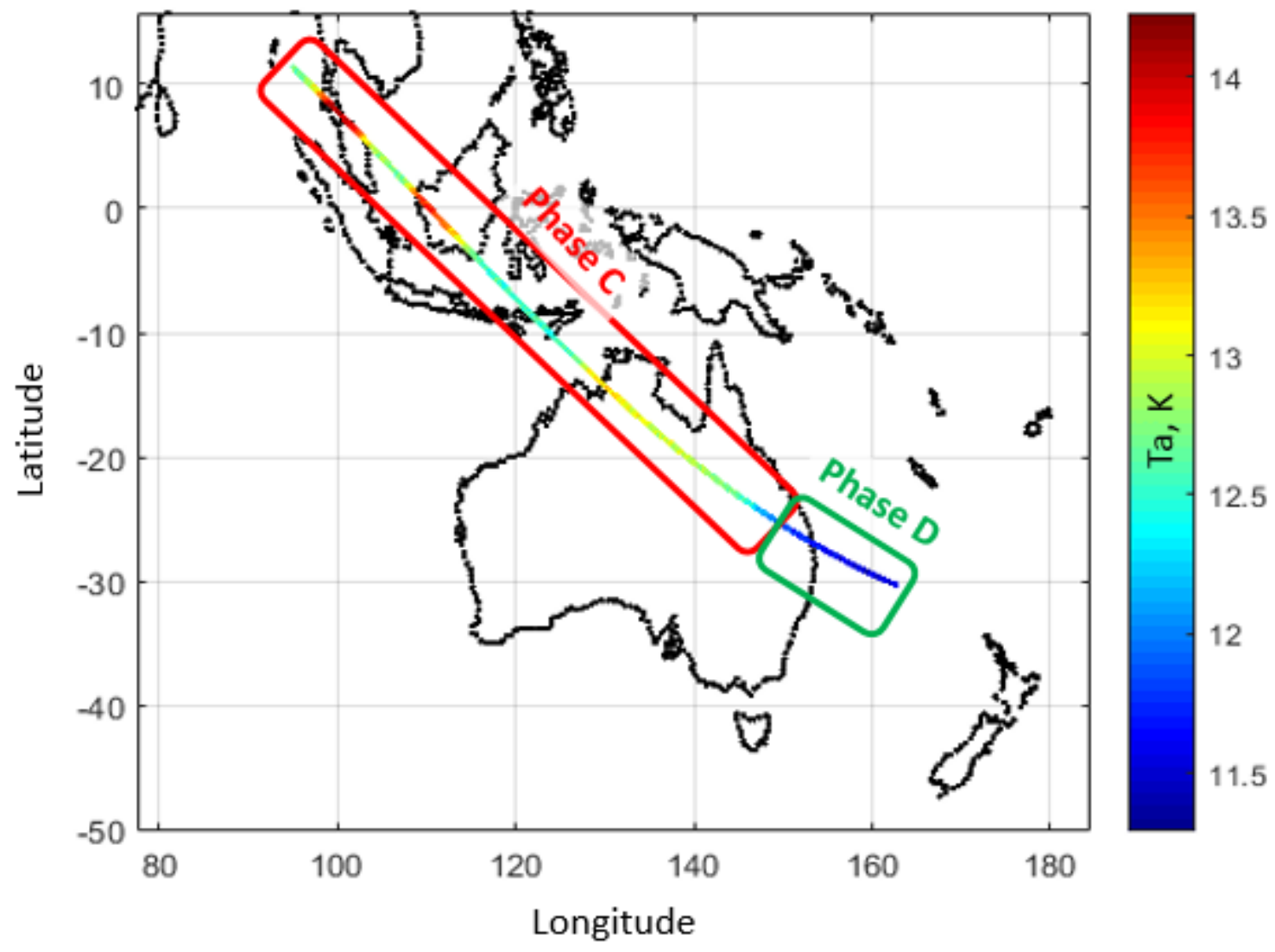

Figure 4.5: Measured antenna temperature (10.65V) during the DSM\#18 plotted as a colored trace on the TRMM ground track.

\section{$\underline{4.2 \text { Emissivity Coefficients }}$}

TMI antenna brightness temperature during the fourth phase-D represents the CMB temperature, plus the emissive reflector contribution of brightness. Since the CMB temperature is known, the channel emissivity coefficient can be expressed as:

$$
\varepsilon_{i}=\frac{<T_{\text {scene }, i}>-T_{c, i}}{\left(1-\eta_{i}\right)\left(T_{p h y}-T_{c, i}\right)}
$$


where $\left\langle T_{\text {scene }, i}\right\rangle$ is the mean antenna temperature of channel $i$ during phase-D (after removing the spill-over beam component of brightness that also viewed the CMB) using Equation (2.12), $T_{c, i}$ is the CMB temperature of channel $i, T_{p h y}$ is the physical temperature of the main reflector (MR) and, $\eta_{i}$ is the pre-launch determined antenna spill-over coefficient. The CMB temperature for all low frequency channels $(10.65 \mathrm{GHz}$ to $37 \mathrm{GHz})$ is $2.7 \mathrm{~K}$ while it is $3.2 \mathrm{~K}$ for the $85.5 \mathrm{GHz}$ channels. It is important to note that the spill-over component of the antenna brightness temperature was directly captured by the feed horn pattern without reflection from the emissive main reflector. Thus, removing the spill-over temperature was required before calculating the emissivity coefficient of each channel.

Because TMI did not have a temperature sensor on the MR, it was necessary to use an external source to estimate the $T_{p h y}$ required in Equation (4.3). For this purpose, another conical scanning satellite radiometer, the Global Precipitation Mission Microwave Imager (GMI), was used [4]. Both GMI and TMI used VDA coated graphite-epoxy parabolic reflectors of similar construction. Because both TRMM and GPM satellites flew in low inclination (non-sun synchronous) orbits, it was possible to match the sun beta angle and the time since eclipse to estimate the $T_{p h y}$. While, there is no evidence for the exact matching of TMI and GMI reflector temperatures, because of the similar reflector construction and the similar day/night orbits, it is reasonable to expect similar orbital $T_{p h y}$ cycles. Moreover, the derived emissivity coefficients are insensitive to the mean $T_{p h y}$ value. For example, if the $T_{p h y}$ estimate has a $+5 \%(\sim 15 \mathrm{~K})$ bias, then the derived emissivity value would decrease by $4.8 \%$. However, since the emissive reflector $T_{A}$ correction involves the product of emissivity and $T_{p h y}$, this results in a negligible brightness temperature error $(-0.022 \mathrm{~K}$ for this case). Moreover, in the final analysis, TMI calibration will be 
referenced to GMI; therefore, any bias in TMI's radiometric calibration, will be removed by crosscalibrating with GMI.

Table 4.2 presents the specific scans number, scan positions, and the estimated $T_{p h y}$ for each yaw-90 DSM. And, the corresponding emissivity coefficient for each channel and each DSM are shown in Fig. 4.6. The quadratic fit for each polarization shows that the emissivity coefficients increase with frequency for each polarization, as experimentally described by [5]. The final emissivity coefficients are the mean of all yaw-90 DSMs, and these values are presented in Table 4.3, and these results are compared with the "Wentz emissivity coefficients" [1] used in the previous version $1 \mathrm{~B} 11 \mathrm{~V} 7$.

Table 4.2: Scan number, scan positions, and estimated Tphy for each yaw-90 DSM.

\begin{tabular}{|l|c|c|c|c|}
\hline \multirow{2}{*}{ Maneuver \# } & \multirow{2}{*}{ Scans \# } & \multicolumn{2}{|c|}{ Azimuth Scan Position \# } & \multirow{2}{*}{ Estimated $\boldsymbol{T}_{\boldsymbol{p h y}}$} \\
\cline { 3 - 4 } & & $10.65-37 \mathrm{GHz}$ & $85.50 \mathrm{GHz}$ & \\
\hline $\mathbf{1 7}$ & $2800-2810$ & $70-80$ & $139-159$ & $274.8 \mathrm{~K}$ \\
\hline $\mathbf{1 8}$ & $2812-2821$ & $70-80$ & $139-159$ & $280.4 \mathrm{~K}$ \\
\hline $\mathbf{1 9}$ & $2861-2870$ & $70-80$ & $139-159$ & $279.15 \mathrm{~K}$ \\
\hline $\mathbf{2 0}$ & $2872-2881$ & $70-80$ & $139-159$ & $276.78 \mathrm{~K}$ \\
\hline
\end{tabular}




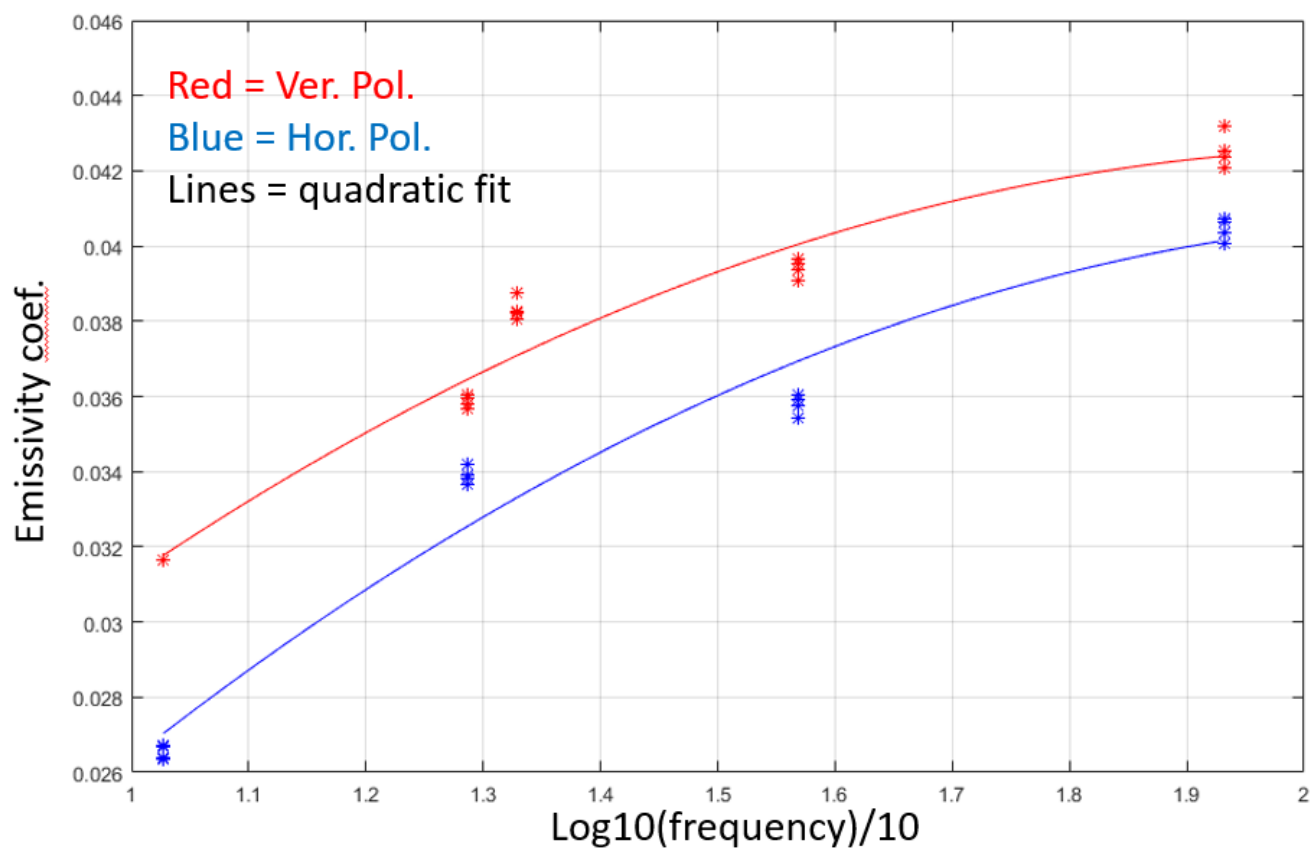

Figure 4.6: Derived main reflector emissivity coefficients for four yaw-90 DSM's.

Table 4.3: Main reflector emissivity coefficients for 1B11 V8 and 1B11 V7

\begin{tabular}{|c|c|c|}
\hline $\mathbf{i}$ & $1 B 11$ V8 & $1 B 11$ V7 \\
\hline $10.65 \mathrm{~V}$ & 0.03163 & 0.038 \\
\hline $10.65 \mathrm{H}$ & 0.02654 & 0.034 \\
\hline $19.35 \mathrm{~V}$ & 0.03586 & 0.042 \\
\hline $19.35 \mathrm{H}$ & 0.03390 & 0.042 \\
\hline $21.30 \mathrm{~V}$ & 0.03832 & 0.046 \\
\hline $37.00 \mathrm{~V}$ & 0.03940 & 0.045 \\
\hline $37.00 \mathrm{H}$ & 0.03580 & 0.042 \\
\hline $85.50 \mathrm{~V}$ & 0.04253 & 0.047 \\
\hline $85.50 \mathrm{H}$ & 0.04045 & 0.044 \\
\hline
\end{tabular}




\section{$\underline{4.3 \text { Physical Temperature During Normal Operation }}$}

After determining the emissivity values, $T_{p h y}$ for each orbit was estimated using the 10.65 V channel ocean brightness temperatures. This channel was selected because the ocean brightness was best known for this channel using the XCAL Radiative Transfer Model (RTM) [12]. This is the result of the lowest and most stable value of atmospheric emission and the smallest change in surface emissivity from wind speed. Thus, the reflector physical temperature was empirically derived to minimize the single differences between the measured and modeled brightness temperatures using the procedure given in Figure 4.7 and using Equation (4.4).

$$
T_{p h y}=\frac{\overline{T_{b, 10.65 V}}}{\varepsilon_{10.65 \mathrm{~V}}}-T_{\text {sim }} \frac{\left(1-\varepsilon_{10.65 \mathrm{~V}}\right)}{\varepsilon_{10.65 \mathrm{~V}}}
$$

where $\overline{T_{b, 10.65 V}}$ is obtained using Equation (2.12), applying the hot load correction.

In Figure 4.7, the simulated brightness temperature $T_{\text {sim }}$ is obtained using the XCAL RTM, which is based on the ocean surface emissivity (Remote Sensing System's ocean surface emissivity model [8]) and the atmospheric absorption coefficients (Rosenkranz and Liebe atmospheric absorption models [7-11]).

The RTM requires environmental input parameters; therefore, the NOAA Global Data Assimilation System (GDAS) data were used. Since the environmental data were gridded in $1^{\circ}$ Lat/Lng boxes, the TMI data for each channel were also gridded in the same way. Moreover, the output $T_{p h y}$ was filtered based on temperature range estimated by GMI; specifically, the filter removed $T_{p h y}$ values that were outside the range $230-320 \mathrm{~K}$.

Since the TMI reflector physical temperature depended on the solar coordinates, the calculated $T_{p h y}$ was saved based on the solar beta angle and the phase from orbit midnight. 
Moreover, the configuration of TRMM (i.e., altitude and yaw direction) is critical on determining the physical temperature; thus, the results were determined separated for each configuration. Finally, TRMM had different articulations of the solar array during the entire mission time: the orbit altitude boost, feathered solar array, and the descending altitude during the end of the mission. Each of these different TRMM situations caused different environments that modulated the MR physical temperature.

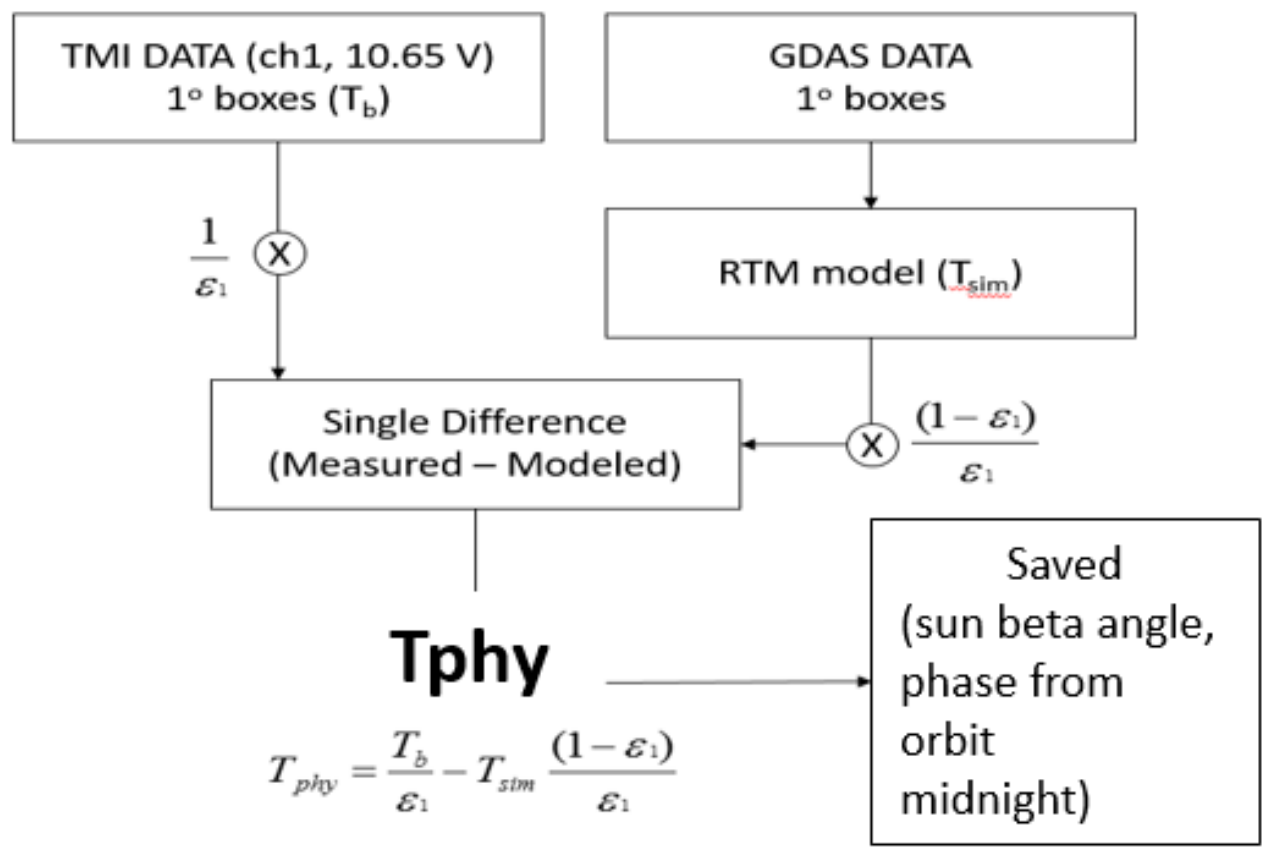

Figure 4.7: Flow chart of the procedure used to estimate TMI MR physical temperature during normal operation.

4.3.1 Main Reflector Physical Temperature (Pre-boost Altitude $=350 \mathrm{~km})$

After obtaining the physical temperature based on the solar beta angle and the phase from orbit midnight, the data were averaged for each orientation (yaw 0 and 180) to create a $2 \mathrm{D} T_{p h y}$ matrix with the following resolution: $1^{\circ} \pm 0.5^{\circ}$ degree in the phase from orbit midnight ( $\mathrm{x}$-axis) and 
$0.25^{\circ} \pm 0.375^{\circ}$ in the solar beta angle (y-axis). Since the environmental profile (GDAS) started in 2000, the 2D MR physical temperature of the pre-boost period was calculated from Jan 2000 to July 2001, as shown in Figure 4.8 (see Appendix E for the results of yaw 180). The 2D matrix was vertically smoothed by triangular filtering, with a window size equal to 17 . This matrix was then used for the TMI counts-to-Tb algorithm, as described in Chapter 2.

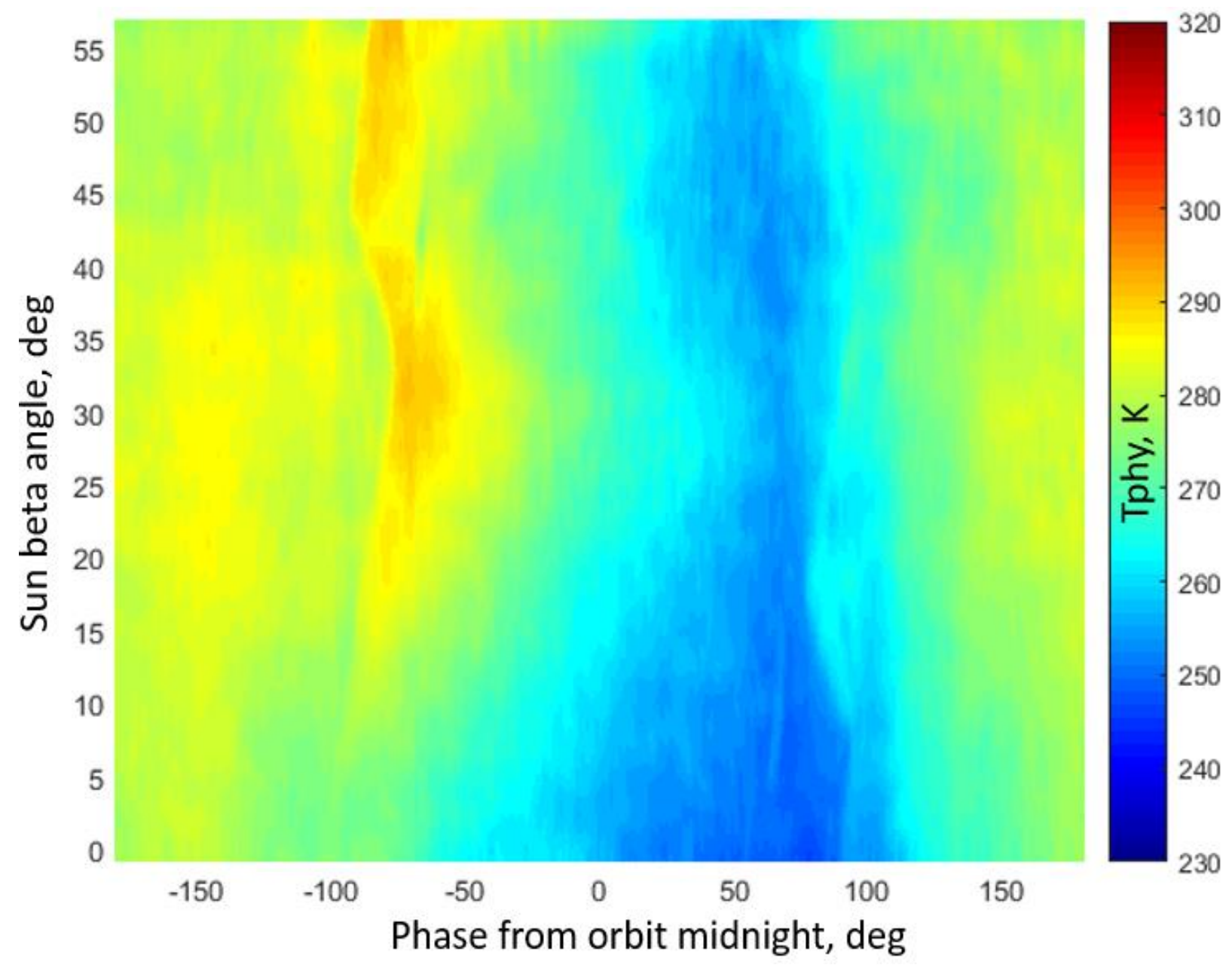

Figure 4.8: 2D matrix of MR physical temperature (yaw 0 and pre-boost altitude $=350 \mathrm{~km}$ ).

An example of the $T_{p h y}$ orbital cycle for a solar beta angle $=43.5^{\circ}$ is displayed versus the time since entering eclipse ( $\mathrm{x}$-axis) in Figure 4.9. At time equal to zero, the satellite enters into the 
night-time of the orbit; thus, the solar heating is removed and the reflector cools rapidly. After 30 minutes, the temperature reaches a minimum value, and as the satellite enters daylight, the temperature exponentially rises. This is a typical example of one orbit thermal cycle, which is modulated by seasonal solar heating (beta angle) that raises and lowers the maximum, minimum and average temperatures. Further, there are frequent, periodic, short-term thermal features, caused by shadows from the spacecraft structure and solar array, that block the solar heating for $5-10$ minute periods during the daylight portion of the orbit. It should be noted that the $T_{p h y}$ orbital cycle is very repeatable (within a few Kelvin) given the beta angle and the phase of the orbit midnight (related to the orbit time since eclipse). Finally, it was determined that the small scale oscillations in $T_{p h y}$ were primarily sampling variability due to the limited (18 month) data available in the preboost period.

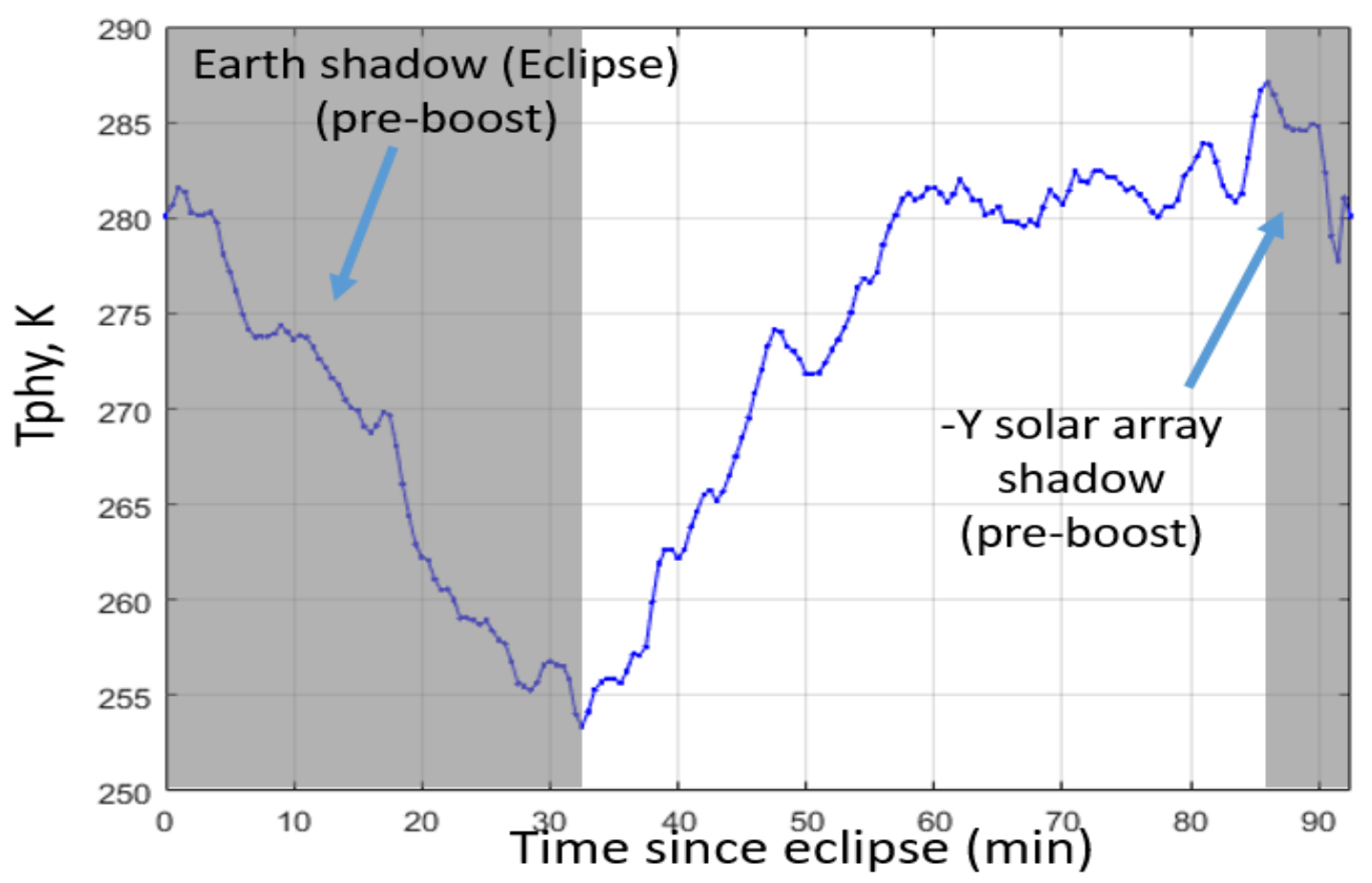

Figure 4.9: Tphy relative to time since eclipse for yaw 0 and pre-boost at solar beta angle $=43^{\circ}$. 


\subsubsection{Main Reflector Physical Temperature (Post-boost Altitude $=405 \mathrm{~km}$ )}

The same procedure that was applied in the pre-boost was used in the post-boost, where the post-boost period was from Aug, 2001 to Aug, 2014. However, the data used in the analysis ranged from Jan, 2003 to Dec, 2013 to avoid the non-feathered solar array or the altitude descending time. The 2D matrix for the post-boost period is shown in Fig. 4.10, using the same color scale as previous.

When comparing the 1D $T_{p h y}$ time series between the pre-boost and post-boost period for the same beta angle, it was determined that the mean physical temperature was $\sim 5 \%$ less in the pre-boost than the post-boost, as seen in Figure 4.11. This is due to the fact that the "-Y solar array" was feathered in the post-boost, which caused the TMI MR to be in daylight longer than in the pre-boost. Although the eclipse time in the pre-boost was slightly shorter than in the post-boost, the solar array shadow was the difference that caused more shadow in the pre-boost period. Further, the small scale fluctuations disappeared in the post-boost because of the much larger quantity of data used to produce the table.

Also, when comparing the 1D Tphy time series between the TMI post-boost and the measured physical temperature of the GMI for the same beta angle, both have similar patterns with slightly different mean values, as shown in Figure 4.12. Although TRMM and GPM orbits have the same altitude, the mechanical structures of the satellites are different. Because of this, the spacecraft shadows the microwave imager differently, which results in differences between the two curves. However, these differences are small and do not influence the emissivity coefficients derivation. 


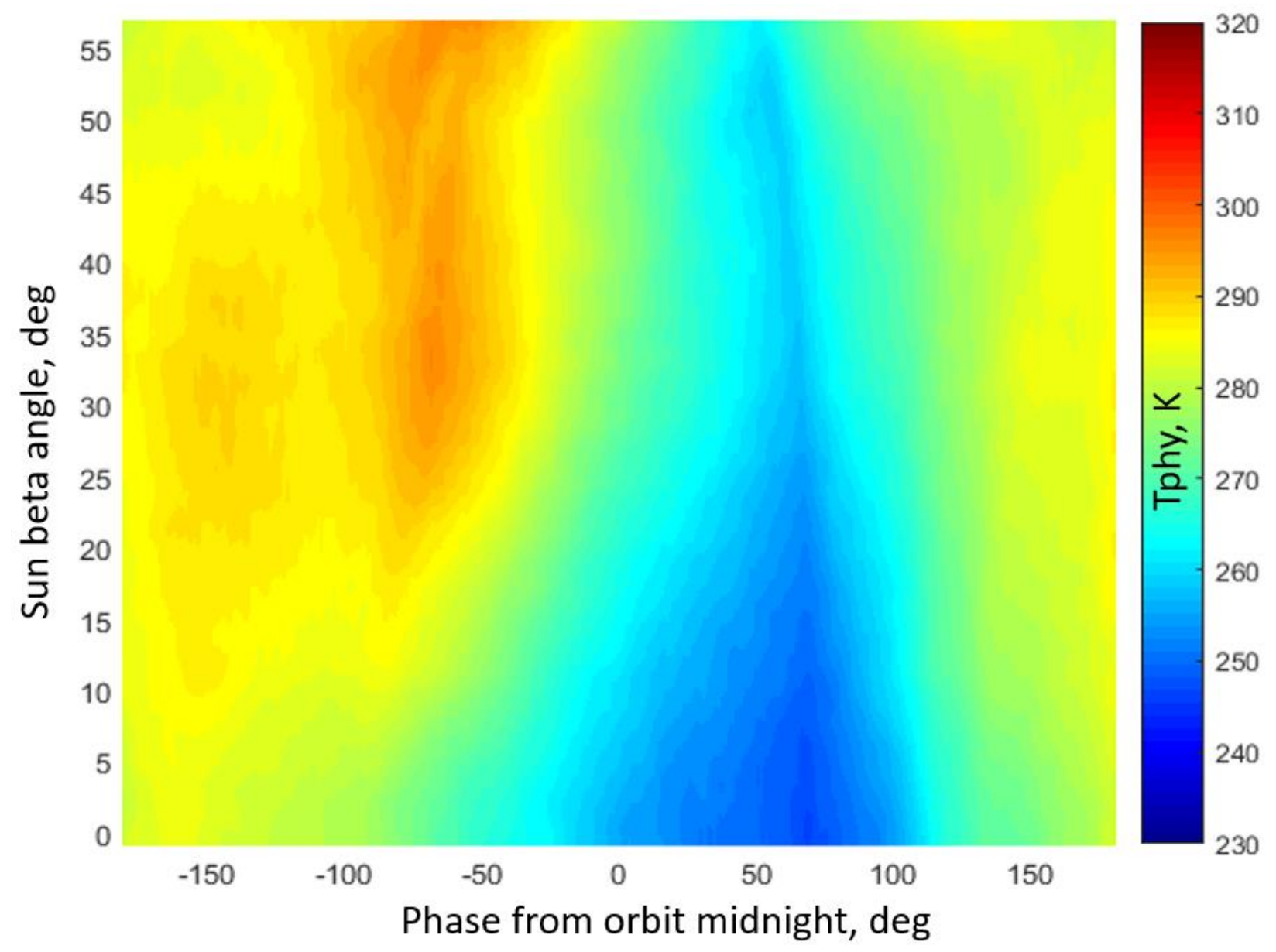

Figure 4.10: 2D matrix of MR physical temperature (yaw 0 and post-boost altitude $=405 \mathrm{~km}$ ). 


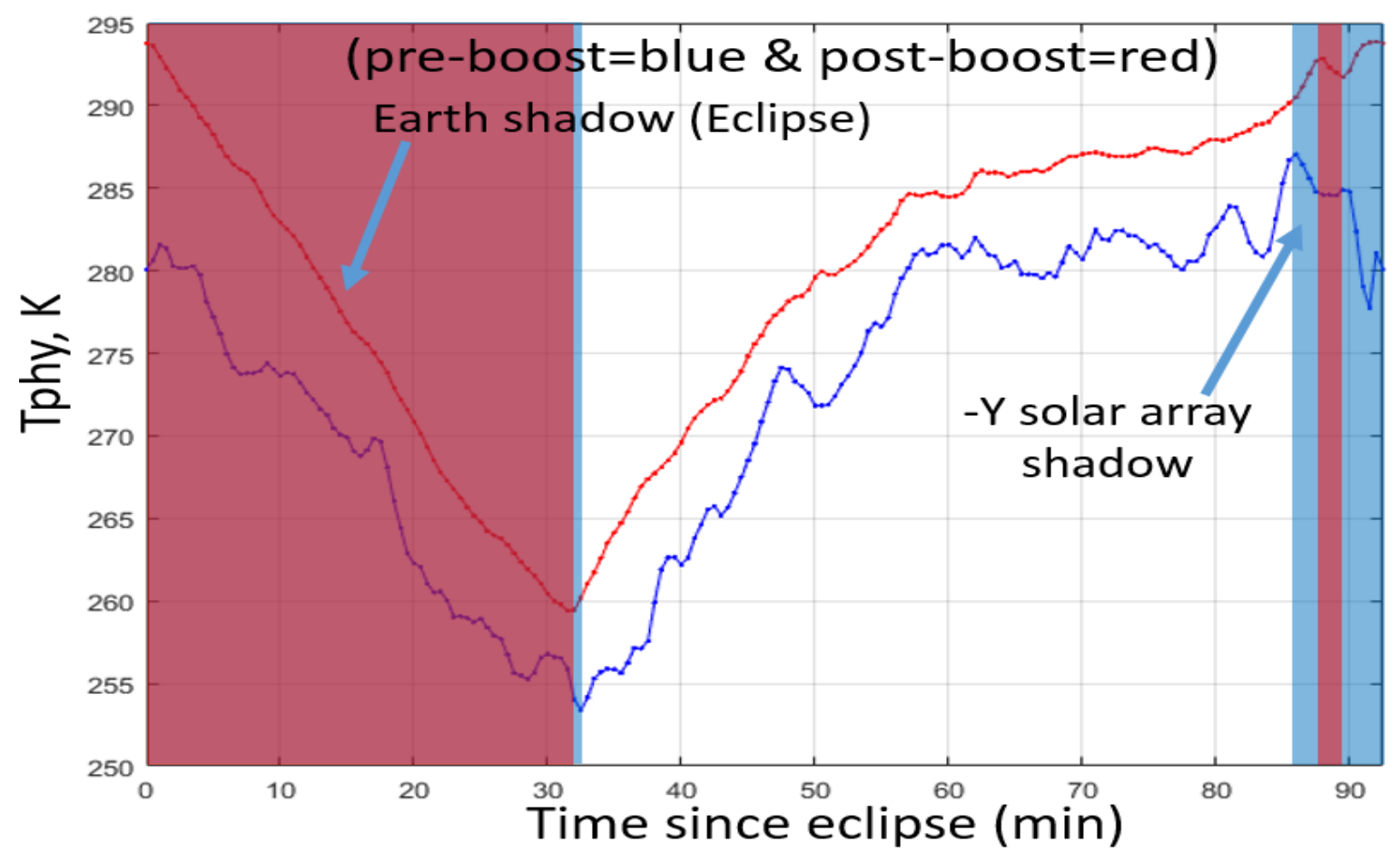

Figure 4.11: Comparing 1D Tphy between pre-boost $\&$ post-boost at solar beta angle $=43^{\circ}$.

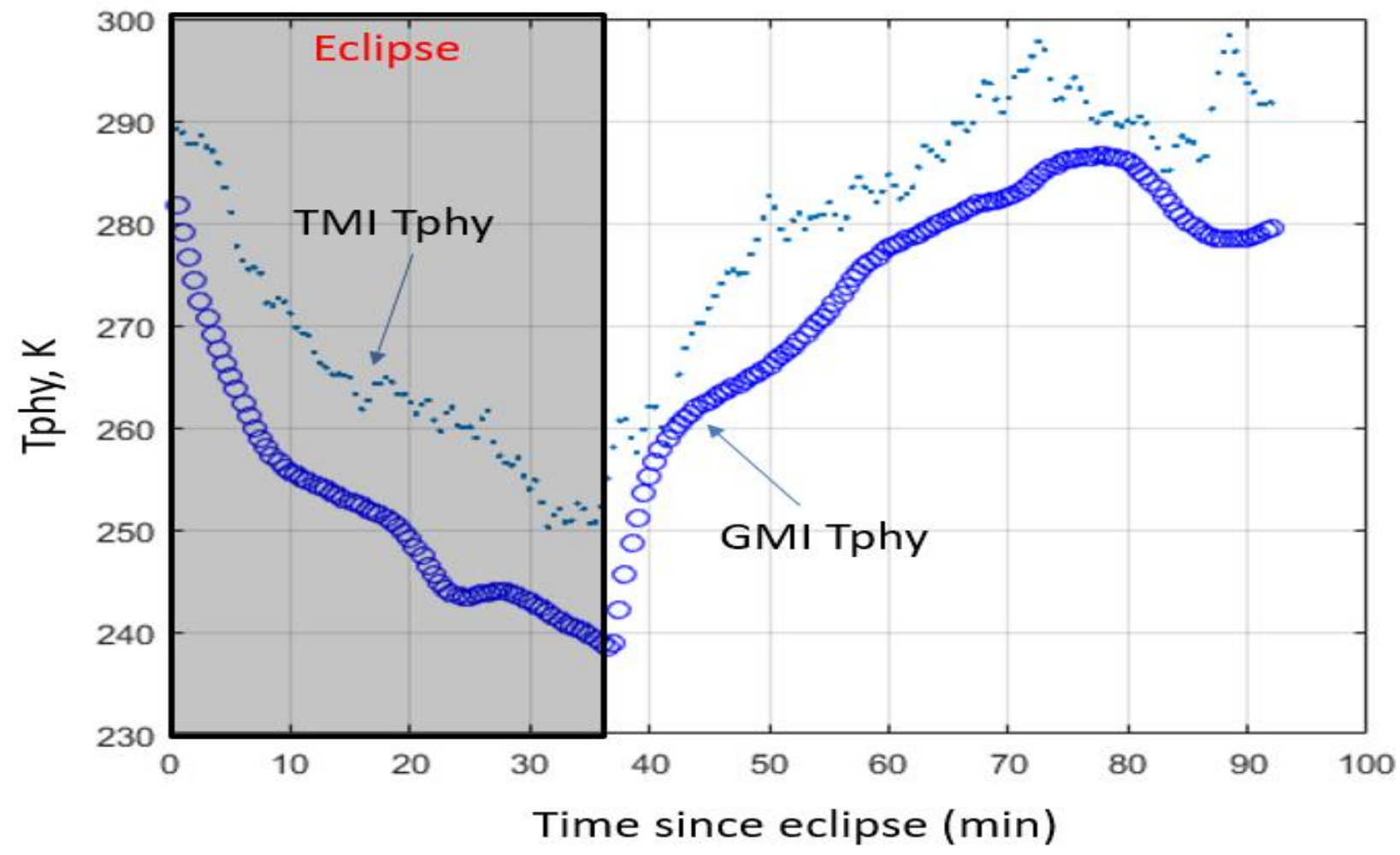

Figure 4.12: Comparison of the post-boost 1D Tphy for TMI and of the measured GMI Tphy at solar beta angle $=43^{\circ}$. 


\subsubsection{Main Reflector Physical Temperature (end of mission)}

When the on-board fuel used for orbit maintenance was depleted, the altitude of TRMM decreased exponentially due to the atmospheric drag, as shown in Fig. 4.13. Since the MR physical temperature depended on the solar coordinates, it was expected that the reduction in altitude would also result in a reduction of the reflector mean temperature. However, during this period, it was observed that the single differences between the measured and the modeled brightness temperatures increased by $0.1 \mathrm{~K}$ after Aug, 2014. One possible reason for the apparent increase in the MR physical temperature was the reduction of the shadow from the feathered solar array. Nevertheless, because there were insufficient Tb samples to estimate the MR physical temperature, the post-boost table was used with an adjustment of the mean by $\sim+3 \mathrm{~K}$ to remove this altitude effect. 


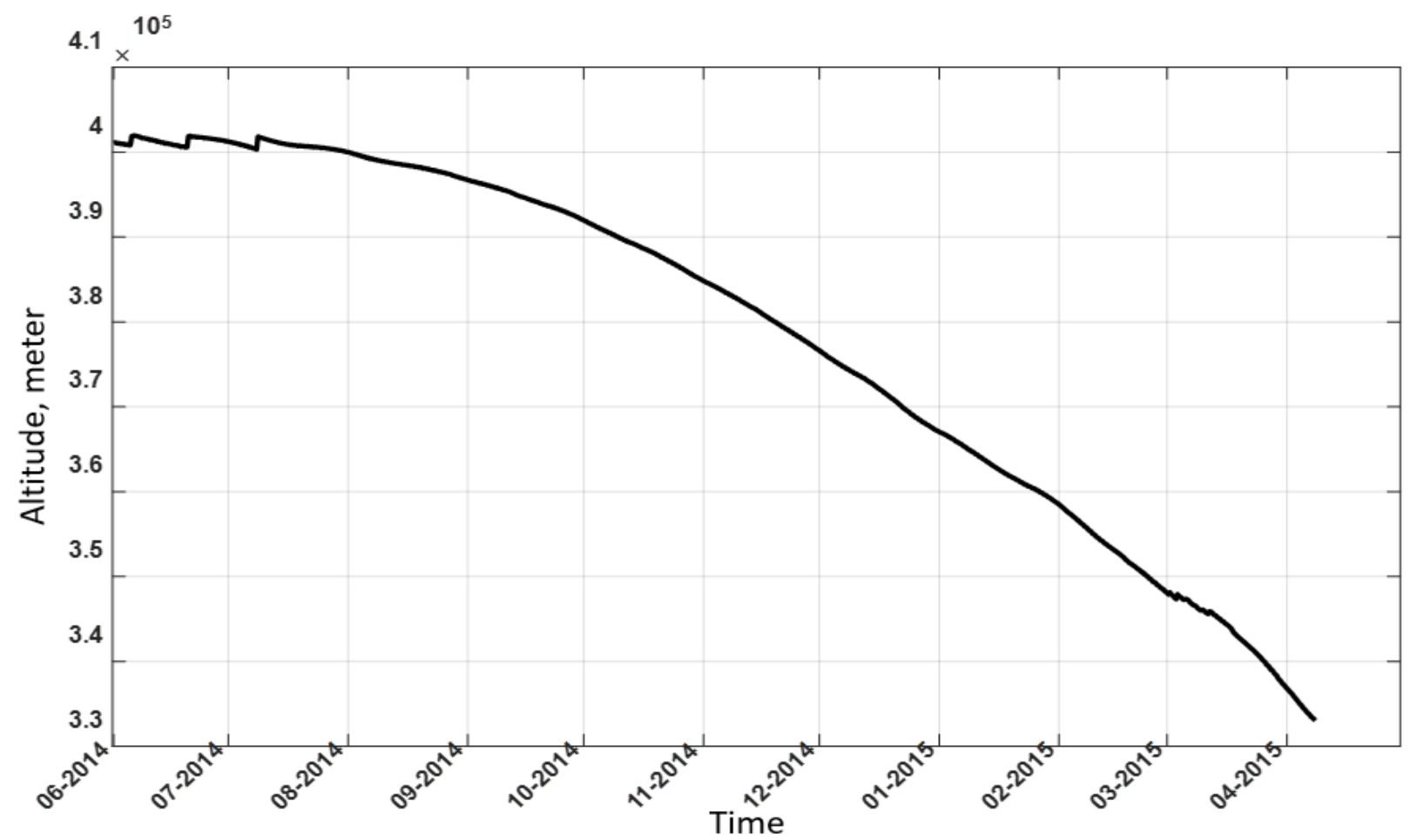

Figure 4.13:TRMM altitude during the last year of mission.

\section{$\underline{4.4 \text { References }}$}

[1] F. J. Wentz, P. Ashcroft, and C. Gentemann, "Post-launch calibration of the TRMM microwave imager," IEEE Transactions on Geoscience and Remote Sensing, vol. 39, no. 2, pp. 415-422, 2001.

[2] F. J. Wentz, "A 17-yr climate record of environmental parameters derived from the Tropical Rainfall Measuring Mission (TRMM) Microwave Imager," Journal of Climate, vol. 28, no. 17, pp. 6882-6902, 2015.

[3] S. Farrar, "An On-orbit Calibration Procedure for Spaceborne Microwave Radiometers Using Special Spacecraft Attitude Maneuvers,” Ph.D. dissertation, Dept. Electrical and Computer Engineering, University of Central Florida, Orlando, FL, 2015. 
[4] D. W. Draper, D. A. Newell, D. S. McKague, and J. R. Piepmeier, "Assessing calibration stability using the Global Precipitation Measurement (GPM) Microwave Imager (GMI) noise diodes," IEEE Journal of Selected Topics in Applied Earth Observations and Remote Sensing, vol. 8, no. 9, pp. 4239-4247, 2015.

[5] S. Swadley, G. Poe, D. Kunkee, W. Bell, S. B. Prata, E. Long, and D. Boucher, "SSMIS calibration anomalies: Observed F-16 \& F-17 Anomalies: Detailed Analysis of the Root Causes and the Path Forward," 16th International TOVS Study Conference, Angra dos Reis, Brazil, 2008.

[6] S. K. Biswas, K. Gopalan, W. L. Jones, and S. Bilanow, "Correction of time-varying radiometric errors in TRMM microwave imager calibrated brightness temperature products," IEEE Geoscience and Remote Sensing Letters, vol. 7, no. 4, pp. 851-855, 2010.

[7] T. Meissner and F. J. Wentz, "The complex dielectric constant of pure and sea water from microwave satellite observations," IEEE Transactions on Geoscience and remote Sensing, vol. 42, no. 9, pp. 1836-1849, 2004.

[8] T. Meissner and F. J. Wentz, "The emissivity of the ocean surface between 6 and $90 \mathrm{GHz}$ over a large range of wind speeds and earth incidence angles," IEEE Transactions on Geoscience and Remote Sensing, vol. 50, no. 8, pp. 3004-3026, 2012.

[9] P. Rosenkranz, "Absorption of microwaves by atmospheric gases," in Atmos. Remote Sensing by Microwave Radiometry, M. A. Janssen, Ed Hoboken, NJ: Wiley, 1993, p. 37.

[10] P. W. Rosenkranz, "Water vapor microwave continuum absorption: A comparison of measurements and models," Radio Science, vol. 33, no. 4, pp. 919-928, 1998. 
[11] H. Liebe, P. Rosenkranz, and G. Hufford, "Atmospheric 60-GHz oxygen spectrum: New laboratory measurements and line parameters," Journal of quantitative spectroscopy and radiative transfer, vol. 48, no. 5-6, pp. 629-643, 1992.

[12] S. K. Biswas, S. Farrar, K. Gopalan, A. Santos-Garcia, W. L. Jones, and S. Bilanow, "Intercalibration of microwave radiometer brightness temperatures for the global precipitation measurement mission," IEEE Transactions on Geoscience and Remote Sensing, vol. 51, no. 3, pp. 1465-1477, 2013. 


\section{CHAPTER 5: 1B11 V8 VALIDATION}

After applying the 1B11 V8 "count to brightness temperature" algorithm described in the previous chapters, the brightness temperatures have been validated by several methods. First, the simulated (modeled) brightness temperatures were used to calculate the single differences (SD) between the observed and the modeled, which is a good indication of the stability of the $\mathrm{Tb}$ measurements. For example, SD's that do not change in time are sufficient to assure a stable radiometric calibration. However, the contrary may not necessarily indicate calibration instability because this SD method is sensitive to uncertainty in the input environmental parameters for atmosphere and surface, as well as issues with the physics of the RTM used to produce the modeled Tb's. Another method, which is more robust, was to use inter-satellite radiometric calibration to calculate the double differences between TMI and other well calibrated instrument. This DD method significantly reduces the issues associated with the SD technique; however, it uses less numbers of points, because it depends on the collocation between two instruments.

\subsection{Stability}

Since TMI operated for more than 17 years, the observed temperature stability is an important metric to be calculated. As discussed above, this was done by analyzing the single differences; where the simulated temperature was obtained using the XCAL ocean RTM. In this dissertation, the RSS surface emissivity model [1, 2], the Rosenkranz atmospheric water vapor absorption model $[3,4]$ and the Liebe atmospheric oxygen absorption model were combined to 
form the RTM [1-5]. The radiometer parameter inputs were: the channel frequency, the Earth Incidence Angle (EIA), and the polarization (V-and H-pol).

The procedure used to calculate the single differences between TMI observed and simulated brightness temperatures is shown in Fig. 5.1. The spatial resolution of the collocation process for TMI Tb's and the environment parameters of GDAS was $1^{\circ}$ for both longitude and latitude. Within each $1^{\circ}$ box, the Tb's were combined and the means and standard deviations were calculated. Afterwards the observed mean Tb's were compared with the simulated $\mathrm{Tb}$ for that box. The environmental parameters for the RTM were provided by NOAA's global numerical weather product GDAS, which began in 2000. Since the GDAS model output is available every 6 hours, there existed $\mathrm{a} \pm 2$ hour window for collocations that occurred 4 times per day.

Since the SD procedure seeks to estimate long-term $\mathrm{Tb}$ biases between radiometers, it is important that the collocations be carefully edited to provide only high quality data for comparisons. The ideal conditions are homogeneous clear-sky ocean conditions, where there is the greatest confidence in the simulated Tb's. As a result, the match-up dataset were subjected to conservative filters, which typically reduce SD comparisons used to $<50 \%$ of the possible collocations. An effective filter used was the standard deviation test, which removes nonhomogeneous environmental conditions that include heavy clouds, rain, and mixed ocean/land scenes [6]. Also, there was a second filter that was based upon a threshold of the maximum observed ocean Tb by channel.

Then, the single differences for each channel were obtained by:

$$
S D_{T M I}=T_{b-o b s}-T_{b-s i m}
$$


The SD for channel 10.65 V are plotted in Figure 5.2, where the values are binned averaged by quarter year that are separated by yaw orientation (yaw 0 and 180 degrees). This channel is the most important because the main reflector Tphy values were derived from these data. During the 15-year time series, from 2000 to the end of the mission, these results show amazing stability where the mean is less than $0.01 \mathrm{~K}$ and the standard deviation is less than $0.07 \mathrm{~K}$ for both orientations. As mentioned above, this result is sufficient to verify that this channel is stable because it is highly unlikely that both the observed $\mathrm{Tb}$ and the modeled $\mathrm{Tb}$ can vary in synchronism to cancel. 


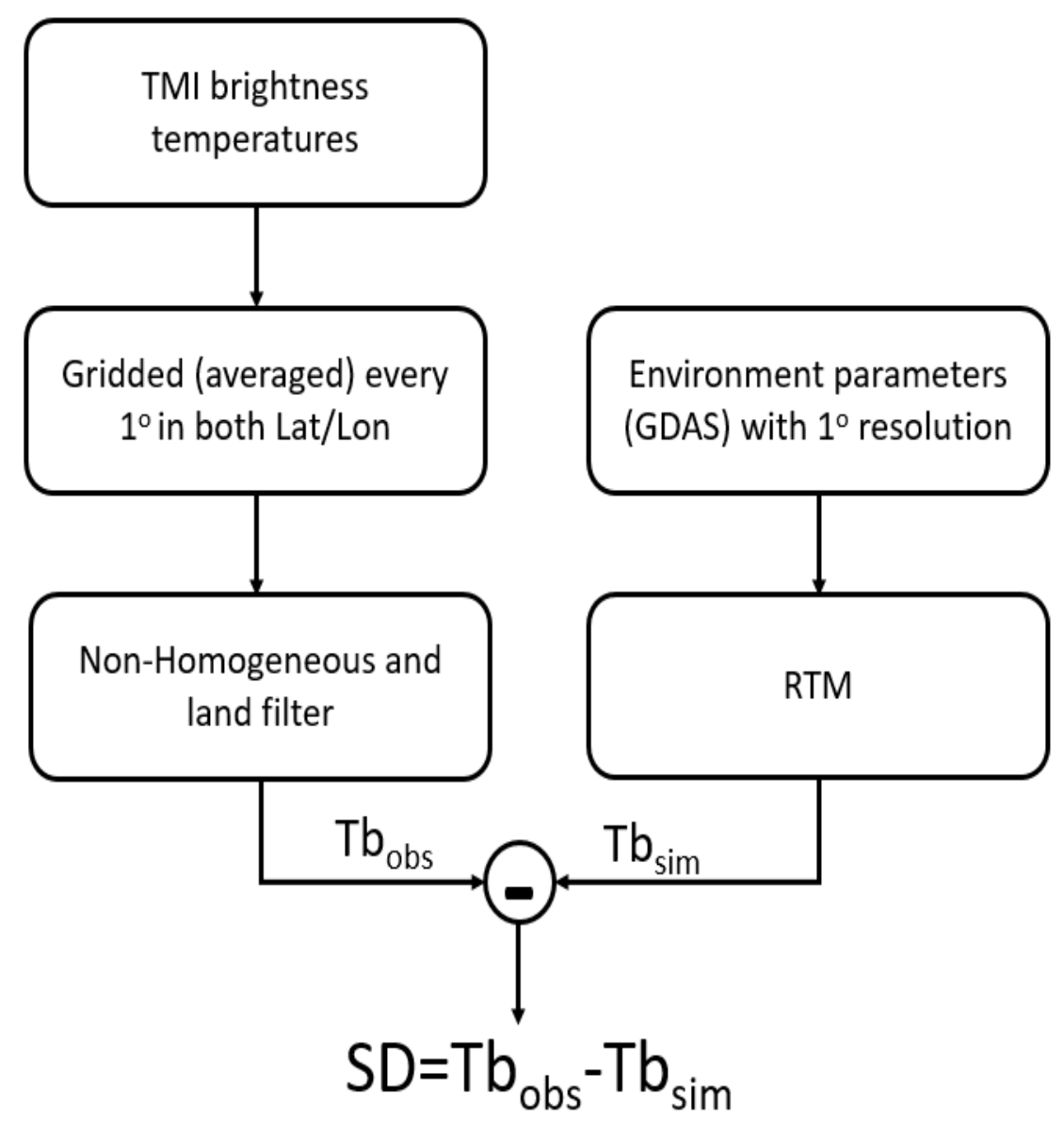

Figure 5.1: The procedure to calculate the Single differences between observed and modeled brightness temperature. 


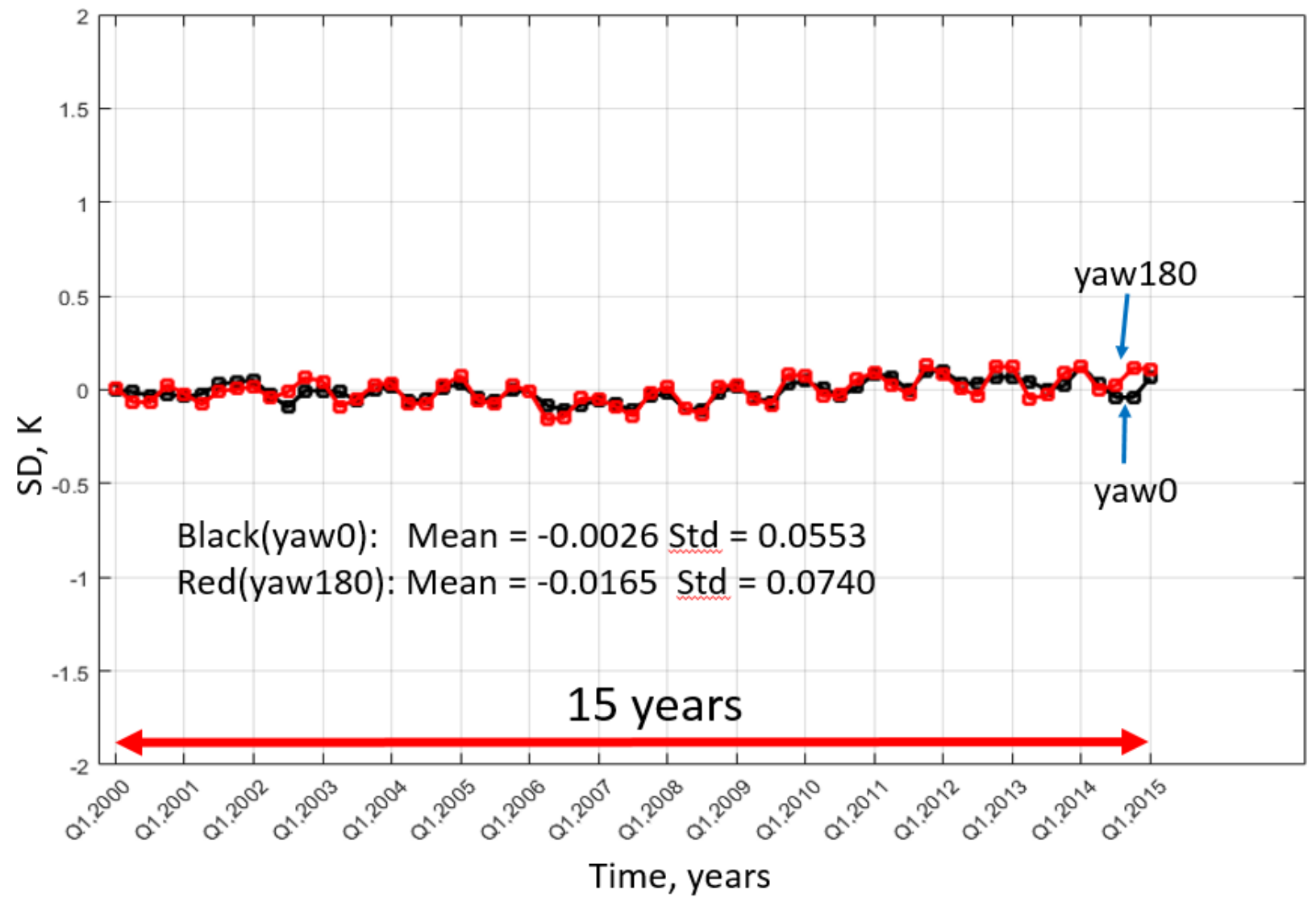

Figure 5.2: The single differences quarterly results for both orientations (yaw 0 and 180 degrees) for 15 years (channel $10.65 \mathrm{~V})$.

\subsection{Double Difference}

Because the GDAS data is only an estimate of the true environmental conditions and the \pm 2-hour collocation time window is relatively large for environmental parameter stability, it is desirable to use another well calibrated instrument for validation and calibration. Because of differences in frequency and EIA between two radiometers, the direct comparison between their observed brightness temperatures does not reflect the true instrument $\mathrm{Tb}$ bias of one to the other. 
However, the difference of the SD's for each instrument, which is called the Double Differences (DD), is a robust technique for establishing the calibration bias [6]:

$$
D D=S D_{\text {reference }}-S D_{\text {target }}
$$

where the well calibrated instrument is the $S D_{\text {reference }}$ while the target instrument to be calibrated is the $S D_{\text {target }}$.

Perhaps the best calibrated microwave radiometers is the Global Precipitation Mission Microwave Imager (GMI), which was launched in Feb 2014 by NASA and JAXA. GMI is a conical-scanning microwave imager that flies in a non-sun synchronous orbit at $405 \mathrm{~km}$ altitude [7]. GMI is the continuation of TMI, with an overlap period of 13 months between them, and although there is some similarity in the center frequency of their microwave channels, as shown in Table 5.1, their EIA's are slightly different.

Table 5.1: Center frequency and bandwidth of TMI and GMI channels.

\begin{tabular}{|c|c|c|c|}
\hline \multicolumn{2}{|c|}{ TMI } & \multicolumn{2}{c|}{ GMI } \\
\hline Frequency (GHz) & Bandwidth (MHz) & Frequency (GHz) & Bandwidth (MHz) \\
\hline $10.65 \mathrm{~V} / \mathrm{H}$ & 100 & $10.65 \mathrm{~V} / \mathrm{H}$ & 100 \\
\hline $19.35 \mathrm{~V} / \mathrm{H}$ & 500 & $18.70 \mathrm{~V} / \mathrm{H}$ & 200 \\
\hline $21.30 \mathrm{~V}$ & 200 & $23.80 \mathrm{~V}$ & 400 \\
\hline $37.00 \mathrm{~V} / \mathrm{H}$ & 2000 & $36.50 \mathrm{~V} / \mathrm{H}$ & 1000 \\
\hline $85.50 \mathrm{~V} / \mathrm{H}$ & 3000 & $89.00 \mathrm{~V} / \mathrm{H}$ & 6000 \\
\hline
\end{tabular}


After calculating the SD for each instrument, the DD are calculated using Equation (5.2). Since the resolution of the SD is $1^{\circ}$ degree box in latitude and longitude, then the DD resolution will be the same. To improve the quality of the inter-calibrations, any match-ups with time difference more than \pm 1 hour were removed.

The standard deviation of the DD values is indicative of the radiometric stability, and the mean DD will be used to calibrate TMI to match GMI. Ideally, the DD biases should be a constant; however, results show some correlation with the yaw orientation, orbital latitude, and the solar coordinates, which will be discussed next.

\subsubsection{TMI/GMI DD results}

The TMI/GMI DD distributions for channel $10.65 \mathrm{~V}$ for versions 6,7 , and 8 are shown in Fig. 5.3 (results of all channels are shown in Appendix F). Consider first the version 6 histogram, shown in red, where the major change was to remove the mean emissive antenna bias. This bias adjustment was based on an ad-hoc procedure involving several months of inter-satellite comparisons, but the MR physical temperature was incorrectly assumed to be constant. Thus, the orbital cycle of MR physical temperature produced in a time-varying radiometric calibration error in the earth scene brightness temperature that resulted in the large histogram variance.

Next, consider the version 7 histogram, shown in blue. This version was an addition to version 6 that removed the effects of the orbital cycle of the MR physical temperature. Thus, the histogram mean was similar to V6 but the variance was significantly reduced. Also, the basis of the emissive reflector correction were empirical comparisons with theoretical brightness temperatures provided by a RTM. 
Finally, version 8, developed during this dissertation research, is based upon rigorous physical measurements and electromagnet theory, as described in the preceding chapters. This is a very important improvement over version 7 , because the long-time series of $\mathrm{Tb}$ observations have significant application in earth science for the study of global climate change. The credibility of the brightness temperature calibration is an important factor in the utility of the experimental data, and version 8 is superior to all previous versions. Concerning the DD histograms, except for the difference in the mean value, versions 7 and 8 are essentially identical. This means that version 7 provided an excellent $\mathrm{Tb}$ product, but it lacked the creditability because it was based upon ad hoc methods. Further, there is no significance to the version 8 bias being less than version 7; rather it is only necessary that its mean value be accurately known. The mean and standard deviation of each TMI channel for both versions 7 and 8 are listed in Table 5.2, and mean biases will be subtracted from each channel in the XCAL inter-calibration process. 


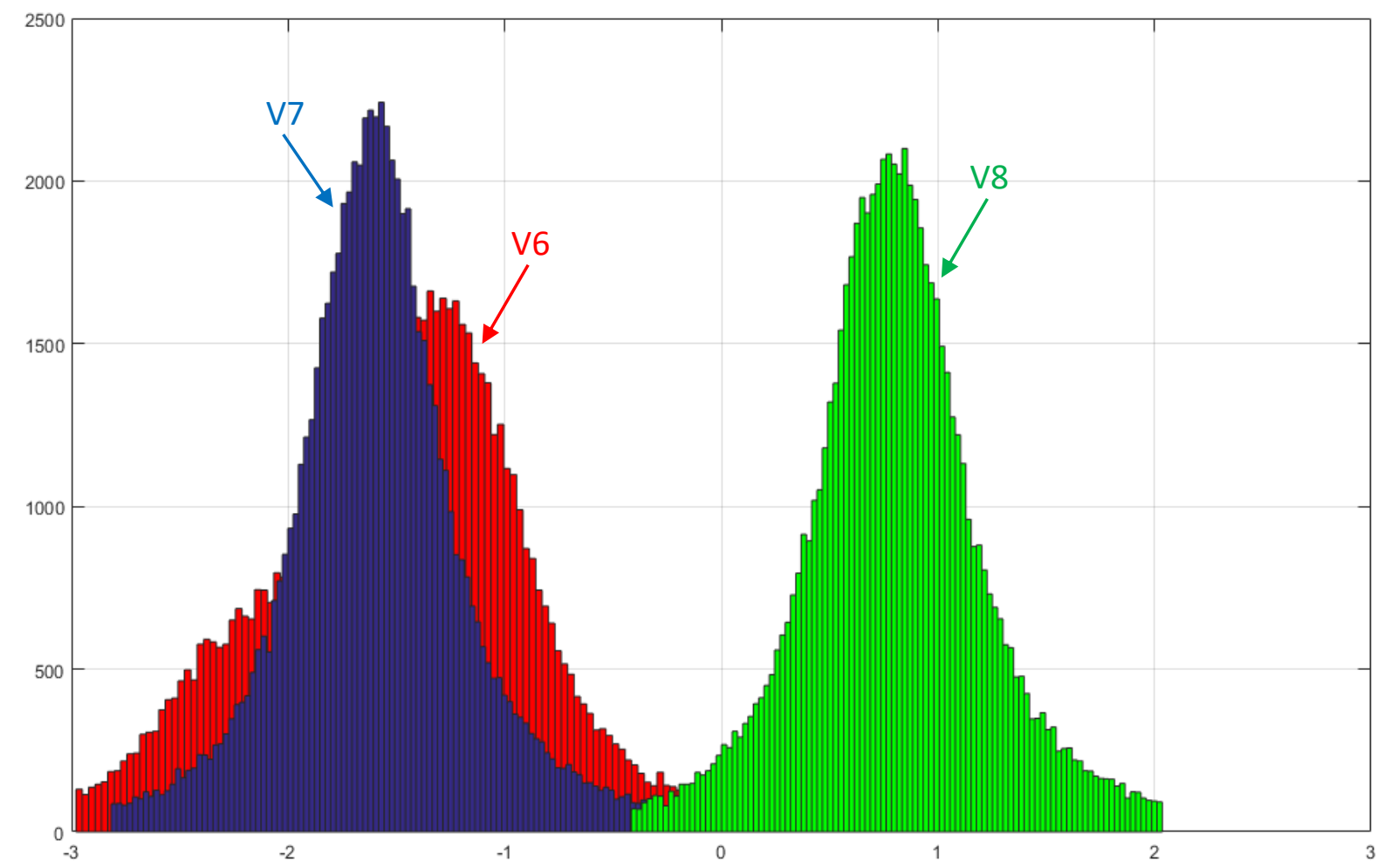

Figure 5.3: DD distribution of channel $10.65 \mathrm{~V}$ for versions 6,7 , and 8 .

Table 5.2:DD values for each channel for both version 7 and 8 .

\begin{tabular}{|l|c|c|}
\hline Channel & Version 8 (mean/std) & Version 7 (mean/std) \\
\hline $\mathbf{1 0 . 6 5}$ V & $0.71 / 0.29$ & $-1.21 / 0.29$ \\
\hline $\mathbf{1 0 . 6 5}$ H & $0.58 / 0.31$ & $-1.70 / 0.30$ \\
\hline $\mathbf{1 9 . 3 5}$ V & $0.45 / 0.46$ & $0.66 / 0.54$ \\
\hline $\mathbf{1 9 . 3 5}$ H & $1.23 / 0.60$ & $0.15 / 0.71$ \\
\hline $\mathbf{2 1 . 3 0}$ V & $1.07 / 0.52$ & $-0.08 / 0.57$ \\
\hline $\mathbf{3 7 . 0 0}$ V & $-0.76 / 0.45$ & $-2.16 / 0.46$ \\
\hline $\mathbf{3 7 . 0 0}$ H & $1.07 / 0.68$ & $-0.83 / 0.66$ \\
\hline $\mathbf{8 5 . 0 0}$ V & $0.25 / 0.41$ & $-1.25 / 0.40$ \\
\hline $\mathbf{8 5 . 0 0}$ H & $-0.60 / 0.69$ & $-1.32 / 0.67$ \\
\hline
\end{tabular}


5.2.2 DD and the yaw orientation

One metric for the DD biases, is the stability of the mean value when data are sorted in various ways, and previously [8], version 7 results were compared for the two yaw orientations. These comparisons are presented (with present results for version 8) in Fig. 5.4. The observed difference in the radiometric bias between yaw orientations is small (typically a few tenths of Kelvin) in version 7; and results for version 8 are even less dependent. One interesting observation is that the DD biases are "mirror images" (opposite polarity) with yaw orientation.

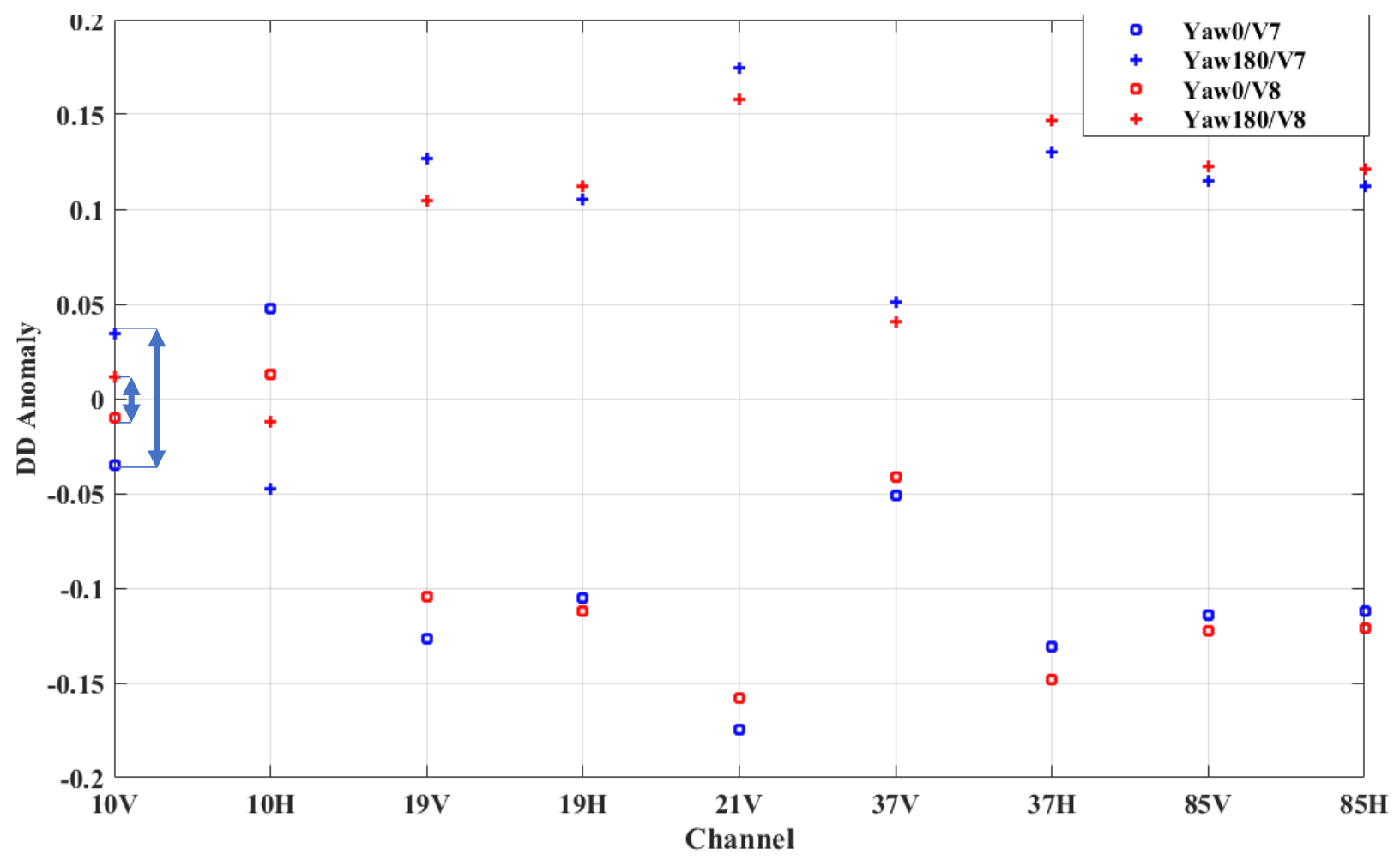

Figure 5.4: Mean of DD of each channel and each yaw orientation for both version 7 and 8 . 


\subsubsection{DD and latitude}

Continuing the analysis of DD, it was noticed that there was a slight dependence on the latitude of the $\mathrm{Tb}$ observations, especially for channel $19.35 \mathrm{H}$. While the reason for this is not understood, it is suspected that it may be related to the global distribution of water vapor. Figure 5.5 shows the DD variation, for both version 7 and 8 , is within the acceptable range of $\pm 0.1 \mathrm{~K}$, with the notable exception of the $19 \mathrm{GHz}$ channels. The DD latitude dependence shows some improvement in version 8, and significant improvements for the $19 \mathrm{GHz}$ channels.

\subsubsection{DD and solar coordinates}

Since the correction of the emissive antenna and the sun intrusion to the hot load were based on the solar coordinates, it will be interesting to see the DD differences between version 7 and 8. To calculate the DD anomaly, the bias should be removed from each version as follows:

$$
2 D D_{\text {anomaly }}(\beta, \text { phase })=\left(D D_{8}-<D D_{8}>\right)-\left(D D_{7}-<D D_{7}>\right)
$$

where $D D_{7}$ and $D D_{8}$ are the DD for version 7 and 8 respectively. The resulting $\left(2 D D_{\text {anomaly }}\right)$ was stored based on the solar coordinates, which are the sun beta angle and the phase from orbit midnight.

The result for channel $19.35 \mathrm{~V}$ and yaw 0 orientation is shown in Fig. 5.6, which includes relatively large differences $(> \pm 0.4 \mathrm{~K})$, especially in the region of the solar intrusion into the hot load. Also, a scatter plot between the GMI earth scene brightness temperatures and the values of $\mathrm{DD}> \pm 0.4 \mathrm{~K}$ is shown in Fig. 5.8. From this figure, version $8 \mathrm{DD}$ appear to be independent of the scene brightness, while version 7 has a significant positive linear dependence. However, not all 
channels show the same improvement. For example, channel $37.00 \mathrm{~V}$ is slightly worse in version 8 (see all results in Appendix F). 

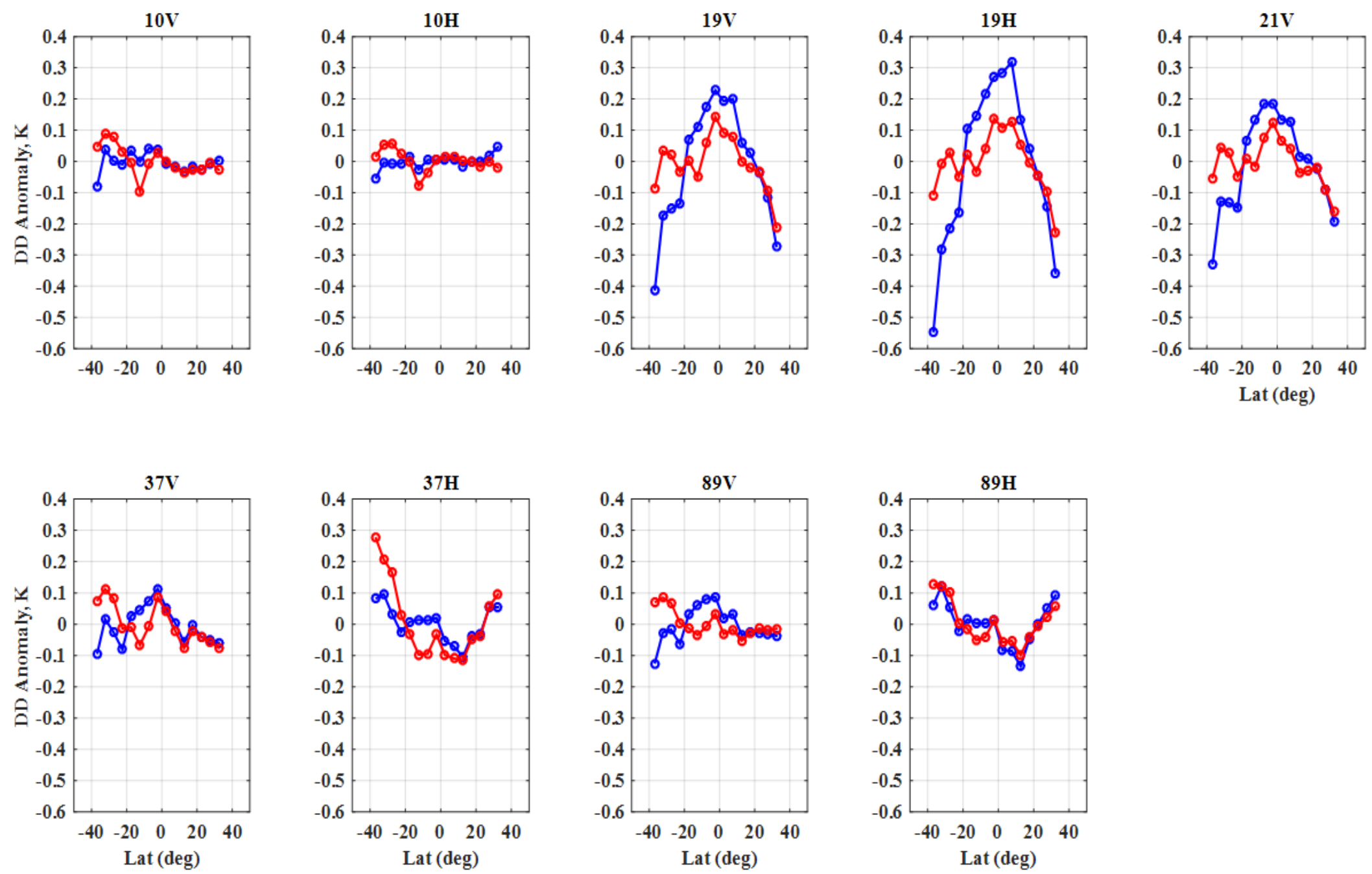

Figure 5.5: DD depends on earth's latitude for versions 7 (blue) and 8 (red). 


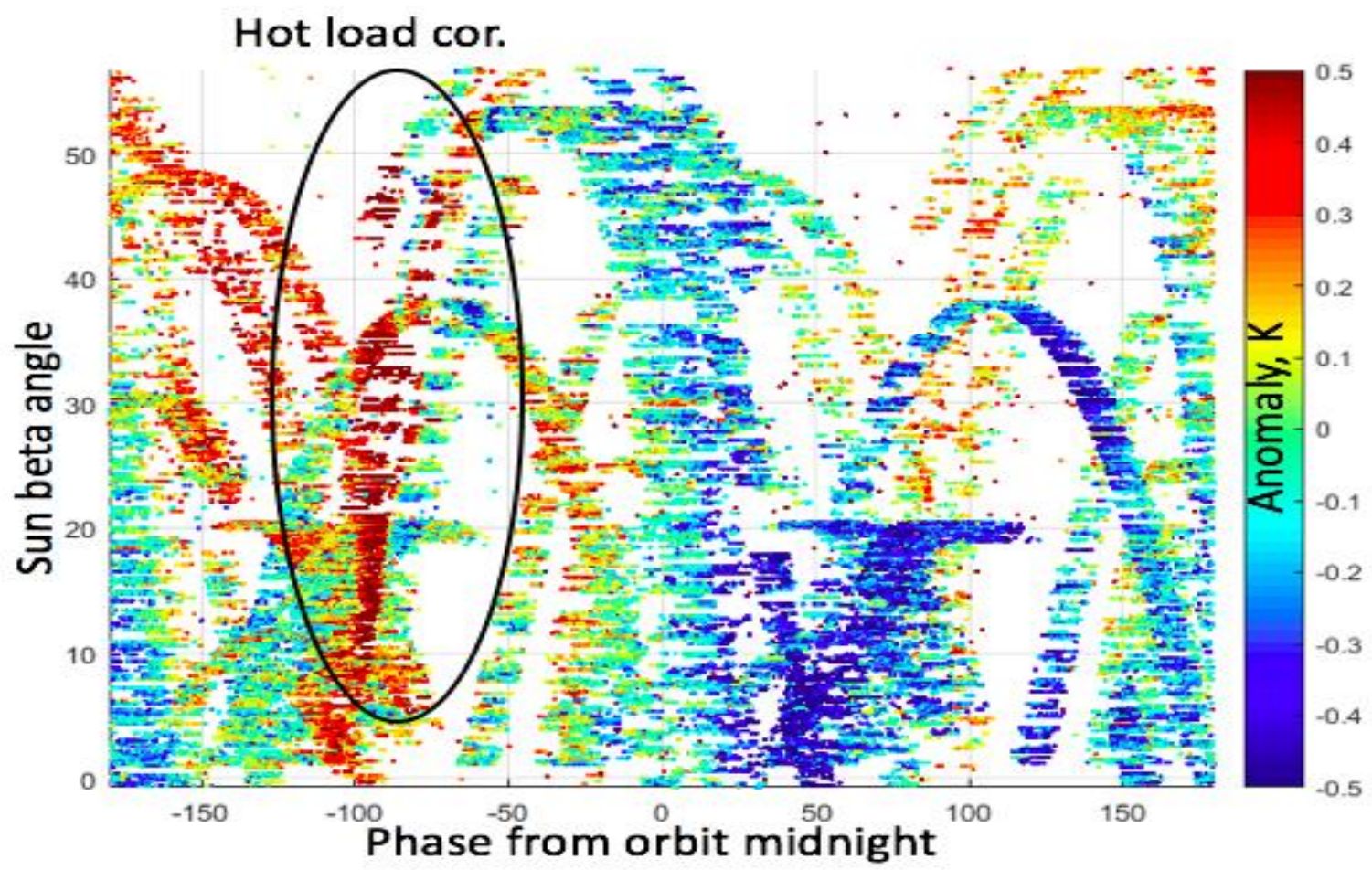

Figure 5.6: DD anomaly between version 7 and 8 based on the solar coordinates of channel 19.35 V.

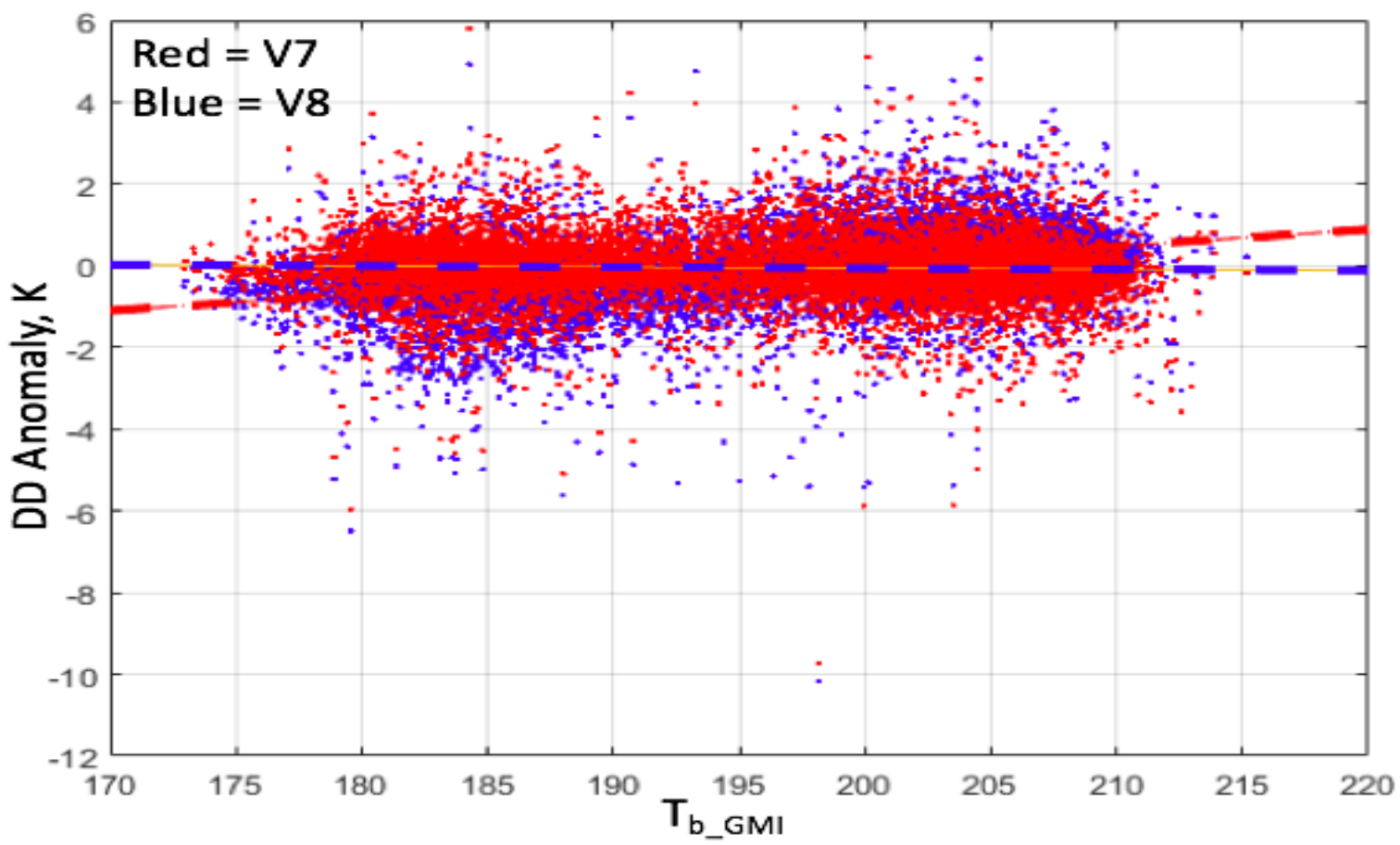

Figure 5.7: Correlation of 19.35 V DD bias with the GMI earth scene brightness temperature for versions 7 (red) and 8 (blue). 


\section{$\underline{5.3 \text { References }}$}

[1] T. Meissner and F. J. Wentz, "The complex dielectric constant of pure and sea water from microwave satellite observations," IEEE Transactions on Geoscience and remote Sensing, vol. 42, no. 9, pp. 1836-1849, 2004.

[2] T. Meissner and F. J. Wentz, "The emissivity of the ocean surface between 6 and $90 \mathrm{GHz}$ over a large range of wind speeds and earth incidence angles," IEEE Transactions on Geoscience and Remote Sensing, vol. 50, no. 8, pp. 3004-3026, 2012.

[3] P. Rosenkranz, "Absorption of microwaves by atmospheric gases," in Atmos. Remote Sensing by Microwave Radiometry, M. A. Janssen, Ed Hoboken, NJ: Wiley, 1993, p. 37.

[4] P. W. Rosenkranz, "Water vapor microwave continuum absorption: A comparison of measurements and models," Radio Science, vol. 33, no. 4, pp. 919-928, 1998.

[5] H. Liebe, P. Rosenkranz, and G. Hufford, "Atmospheric 60-GHz oxygen spectrum: New laboratory measurements and line parameters," Journal of quantitative spectroscopy and radiative transfer, vol. 48, no. 5-6, pp. 629-643, 1992.

[6] S. K. Biswas, S. Farrar, K. Gopalan, A. Santos-Garcia, W. L. Jones, and S. Bilanow, "Intercalibration of microwave radiometer brightness temperatures for the global precipitation measurement mission," IEEE Transactions on Geoscience and Remote Sensing, vol. 51, no. 3, pp. 1465-1477, 2013.

[7] D. W. Draper, D. A. Newell, F. J. Wentz, S. Krimchansky, and G. M. Skofronick-Jackson, "The global precipitation measurement (GPM) microwave imager (GMI): Instrument overview and early on-orbit performance," IEEE Journal of Selected Topics in Applied Earth Observations and Remote Sensing, vol. 8, no. 7, pp. 3452-3462, 2015. 
[8] R. Chen, F. Alquaied, H. Ebrahimi, and W.L. Jones, "Radiometric Validation of The TRMM Microwave Imager 1B11 V8 Brightness Temperature Product," Proc. IEEE International Geoscience and Remote Sensing (IGARSS), Fort Worth, USA, July 23-28, 2017, accepted for publication. 


\section{CHAPTER 6: CONCLUSION AND FUTURE WORK}

Under this dissertation, we have found conclusive evidence of the existence of a slightly emissive coating on the TRMM Microwave Imager main reflector antenna. Prior to this research, the existence of such an emissive antenna was only a plausible hypothesis, and proving this hypothesis has opened the way for the development of a rigorous electromagnetic solution to solve for the desired earth scene brightness temperature $(\mathrm{Tb})$.

The previous version 7 of the TMI 1B11 brightness temperature product, used empirical estimates of the time varying $\mathrm{Tb}$ error caused by a postulated emissive reflector antenna. The estimates were derived for the $10.65 \mathrm{~V}$ channel by computing the single difference between the observed and modeled antenna temperatures $\left(\mathrm{T}_{\mathrm{A}}\right)$ as a function of the solar beta angle and the time since entering eclipse [1]. Tb error corrections for other TMI channels were scaled versions of the $10.65 \mathrm{~V}$ errors using assumed emissivity values based on the inter-satellite calibrations between SSMI (reference sensor) and TMI. After collecting 8 years of data, delta-Tb lookup tables were constructed to correct the observed $\mathrm{T}_{\mathrm{A}}$ for the time varying emissive antenna bias [2].

Under this dissertation, the first direct measurements of the main reflector emissivity coefficients for the TMI channels were obtained during four deep space calibration maneuvers conducted at the end of TRMM mission. Also, using the derived emissivity for the $10.65 \mathrm{~V}$ channel, the main reflector physical temperature time series was estimated for the entire 17-year period of TMI measurements by comparing observed and theoretical modeled Tb's. While this procedure had some uncertainty in the mean physical temperature, the dynamic temperature change was determined with sufficient precision to achieve a satisfactory brightness temperature correction uncertainty $(< \pm 0.1 \mathrm{~K})$. Fortunately, the reflector physical temperature has been parameterized as 
a function of solar coordinates (solar beta angle and orbit phase) relative to the TRMM spacecraft to provide a look-up table. Also, there are minor perturbations in the direct solar heating of the reflector caused by shadows from the solar array and other parts of the spacecraft structure. Fortunately, the reflector orbital temperature cycle is quite repeatable, given the solar coordinates; and as a result, a number of reflector physical temperature lookup tables have been constructed for fixed spacecraft configurations (spacecraft yaw of $0^{\circ} \& 180^{\circ}$, orbit altitude $(350 \mathrm{~km} \& 405 \mathrm{~km})$, and solar array articulation limits) that cover the full 17-year life time of the TMI. Therefore, given main reflector emissivity for TMI channels and the reflector physical temperature, a radiative transfer model has been developed to correct for the emissive component of brightness temperature.

Moreover, under this dissertation, one other anomalous radiometric calibration issue has been discovered, and an effective mitigation solutions has been developed. This concerns periodic sun intrusion in the hot load (at certain solar coordinates), which results in transient radiometer gain errors. This issue has been solved developing a new hot load correction lookup table, which will be applied on the upcoming TMI version 8 .

Therefore, the procedure of TMI 1B11 version 7 will be replaced a new "radiometer counts to $\mathrm{Tb}$ " processing algorithm that incorporates the above innovations to produce the legacy $\mathrm{TMI}$ 1B11 version 8 . More importantly, this version $8 \mathrm{~Tb}$ product will have an improved pedigree that replaces ad hoc radiometric calibrations (version 7) by a rigorous derivation of radiometric uncertainty, based upon on-orbit measurements and electromagnetic theory (version 8). This increases our confidence in the results and allows earth scientist to apply statistical estimation 
techniques to determine the uncertainty in their earth geophysical models based upon quantitative estimates of $\mathrm{Tb}$ errors provided.

\section{$\underline{6.1 \text { Future Work }}$}

Since the TMI 1B11 version 8 is the last legacy version, there will not be future updates of this important $\mathrm{Tb}$ dataset. However, the rigorous radiometric calibration approach developed under this dissertation can be used for radiometric calibration of future satellite radiometers. In particular, it is recommended that the lessons learned concerning the need to consider the effects of solar coordinates on the instrument calibration be incorporated during the design phase.

Further, the design of deep space calibration maneuvers need to consider the optimum spacecraft attitude adjustment procedures to obtain the desired simultaneous pointing of all radiometer antenna beams into space. As noticed in TMI, the first 16 DSMs, using the conventional pitch rotation maneuver, were limited due to the contamination of earth through the spill-over beam. However, the last four maneuvers were modified by changing the yaw to 90 degrees and apply rolling maneuver instead of pitching maneuver. This caused the TMI antenna beams (main beam, spill-over beam, and the cold sky reflector beam) to look at space simultaneously without any contamination of earth. Thus, applying different maneuver's attitude to other instruments may provide more useful results than only used the typical maneuver which is based on pitch angle. 


\section{$\underline{6.2 \text { References }}$}

[1] K. Gopalan, "A Time-Varying Radiometric Bias Correction for The TRMM Microwave Imager," Ph.D. dissertation, Dept. Electrical and Computer Engineering, University of Central Florida, Orlando, FL, 2008.

[2] S. K. Biswas, S. Farrar, K. Gopalan, A. Santos-Garcia, W. L. Jones, and S. Bilanow, "Intercalibration of microwave radiometer brightness temperatures for the global precipitation measurement mission," IEEE Transactions on Geoscience and Remote Sensing, vol. 51, no. 3, pp. 1465-1477, 2013. 


\section{APPENDIX A: SCAN BIAS CORRECTION}


In this Appendix, the scan bias correction for all TMI channels are presented. While these results are not part of this dissertation, they were used in the derivation of the reflector emissivity and the main reflector physical temperature. Since the scan bias correction is determined by channel number and the antenna "brightness" temperature, the figures will be for each channel with two different scene temperature ranges, which is cover the ocean and land observations.

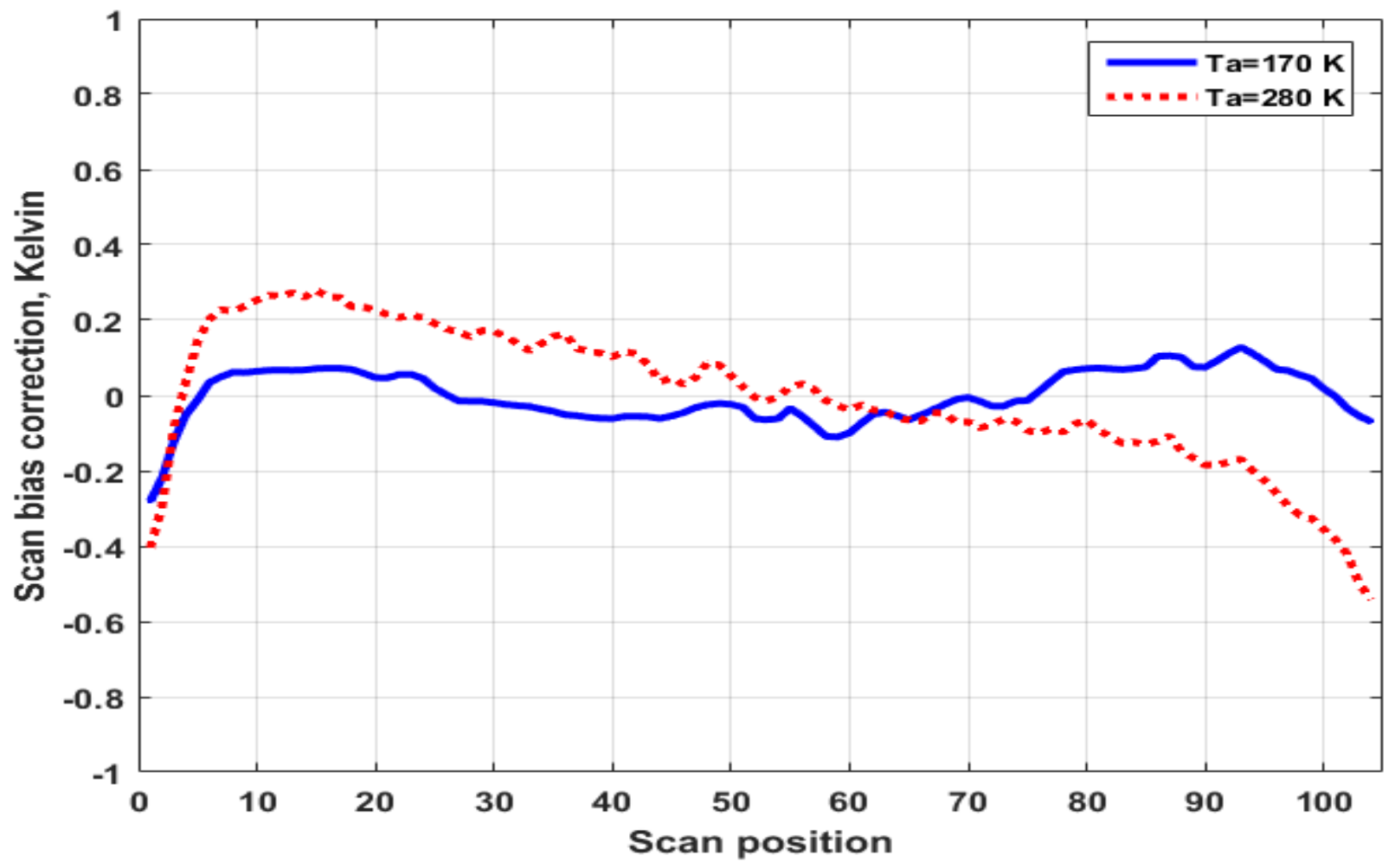

Figure 6.1: Scan bias correction for channel $10.65 \mathrm{~V}$ for $\mathrm{Ta}=170 \mathrm{~K}$ and $\mathrm{Ta}=280 \mathrm{~K}$. 


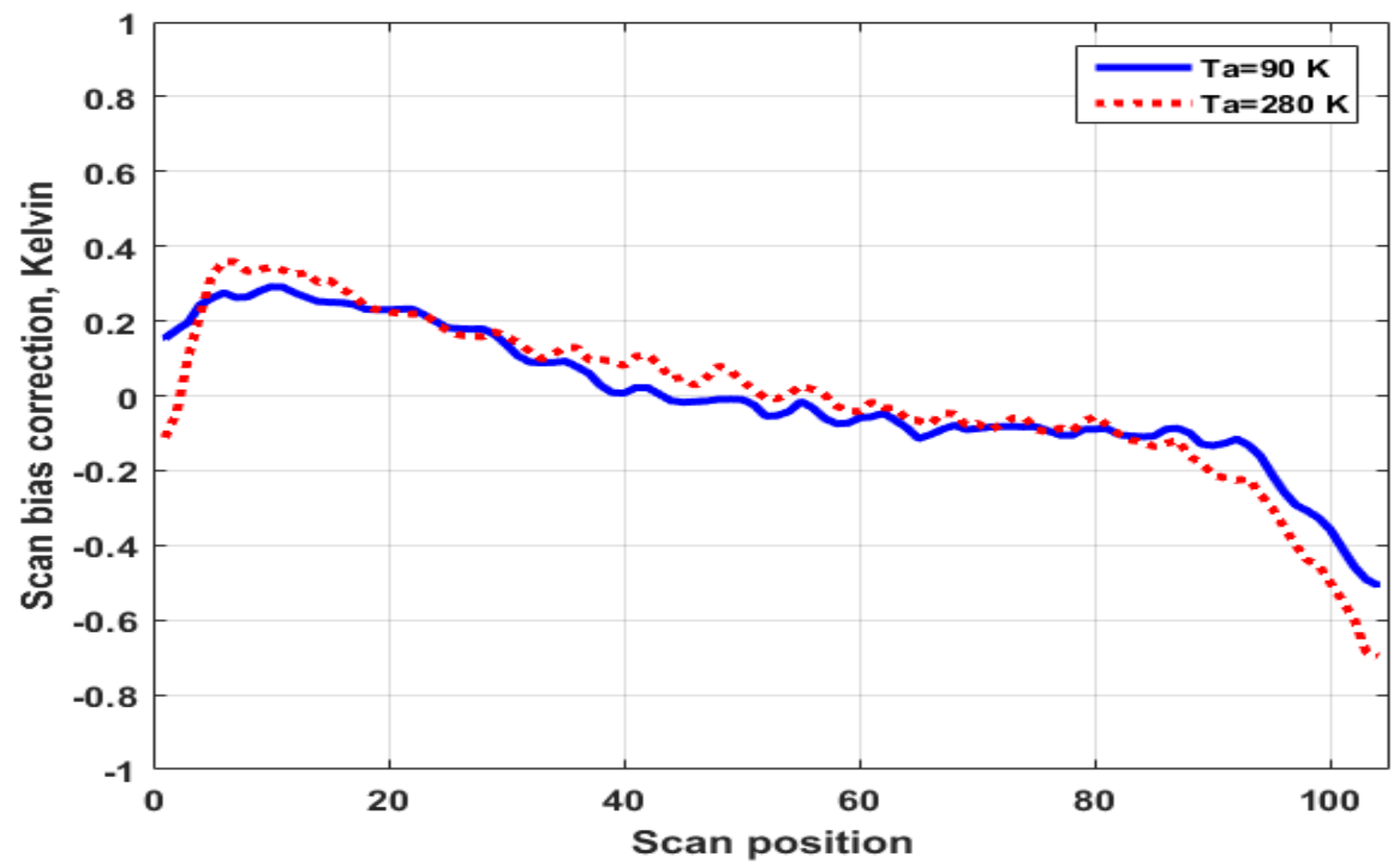

Figure 6.2: Scan bias correction for channel $10.65 \mathrm{H}$ for $\mathrm{Ta}=90 \mathrm{~K}$ and $\mathrm{Ta}=280 \mathrm{~K}$.

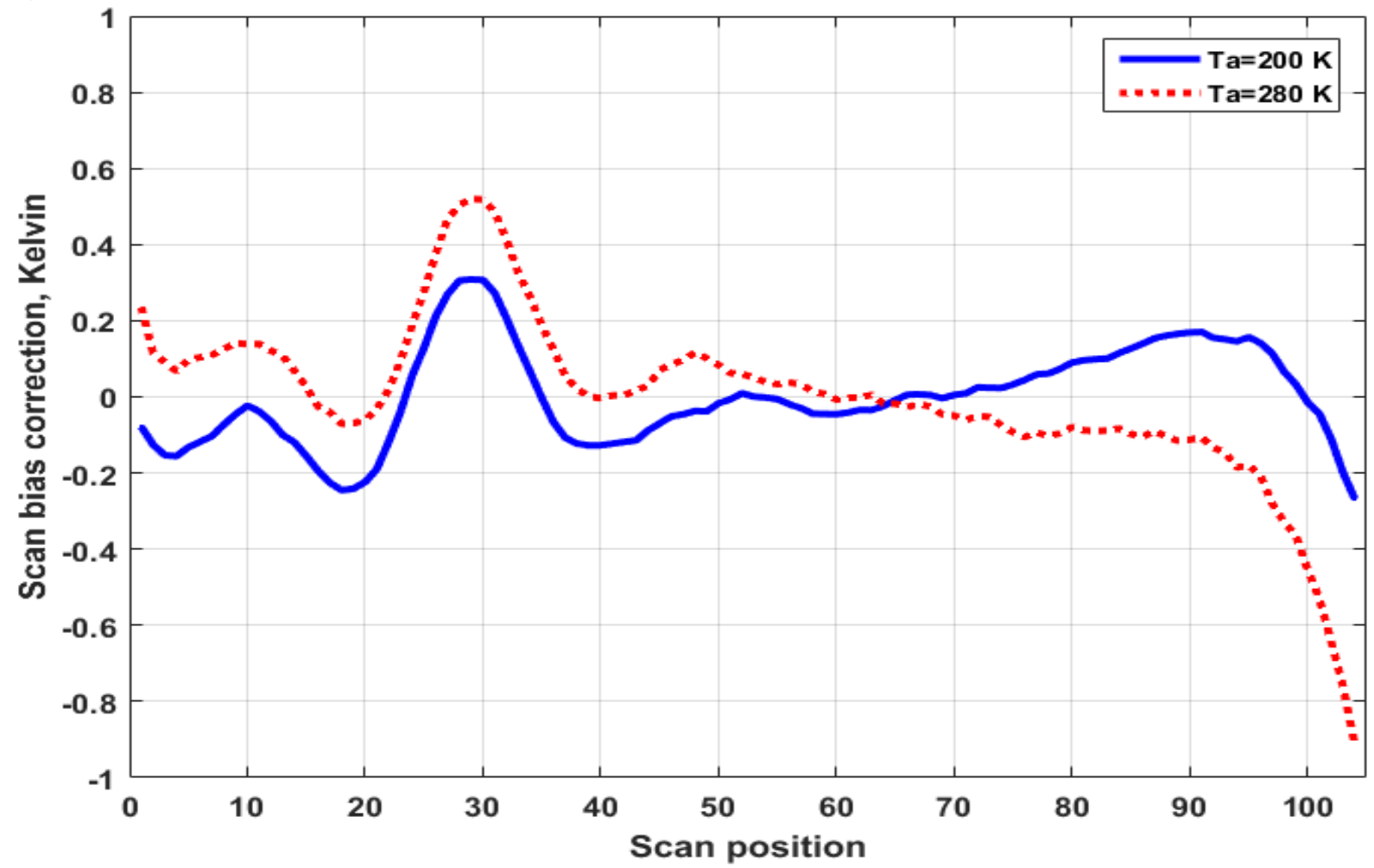

Figure 6.3: Scan bias correction for channel 19.35 V for $\mathrm{Ta}=200 \mathrm{~K}$ and $\mathrm{Ta}=280 \mathrm{~K}$. 


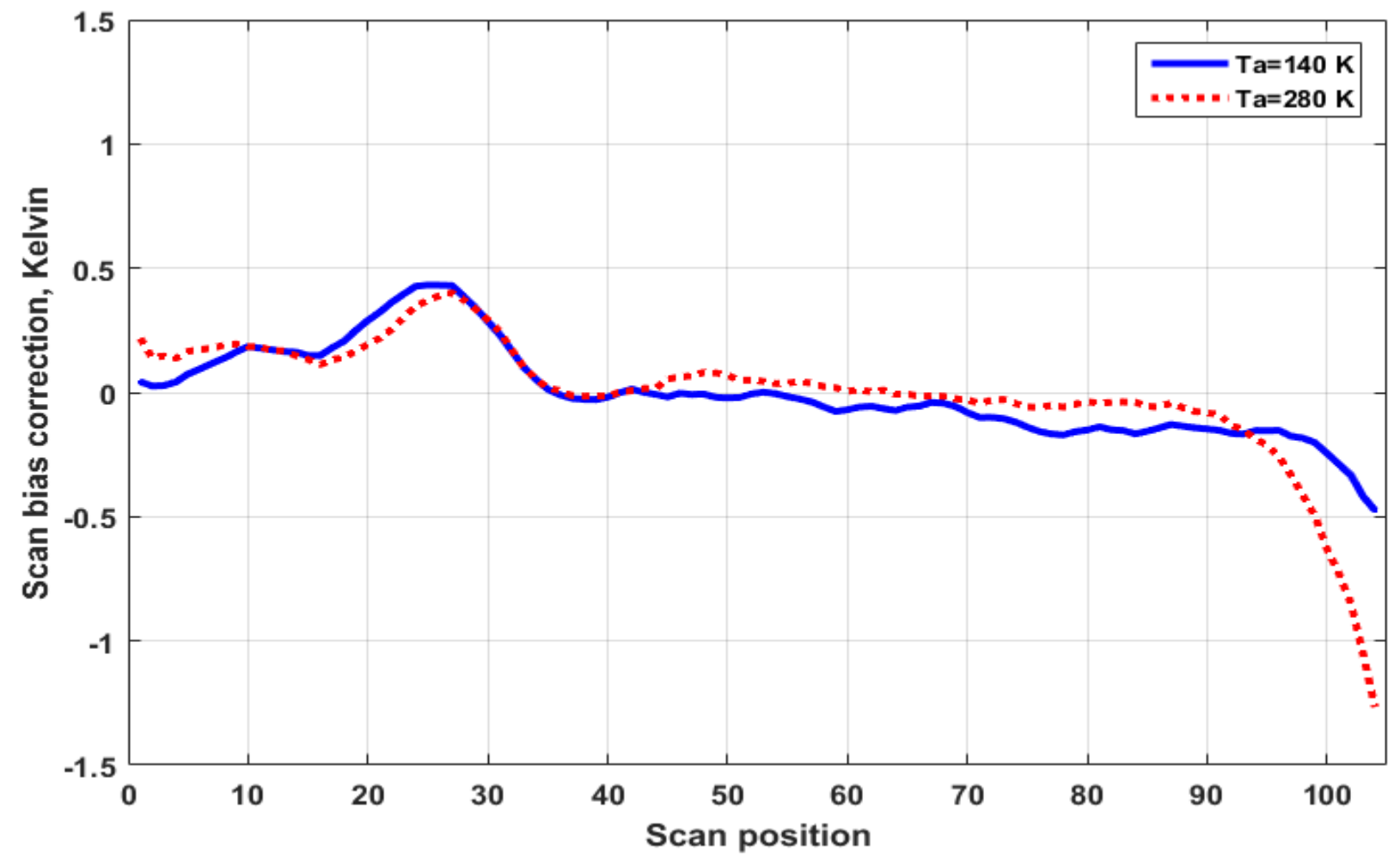

Figure 6.4: Scan bias correction for channel $19.35 \mathrm{H}$ for $\mathrm{Ta}=140 \mathrm{~K}$ and $\mathrm{Ta}=280 \mathrm{~K}$.

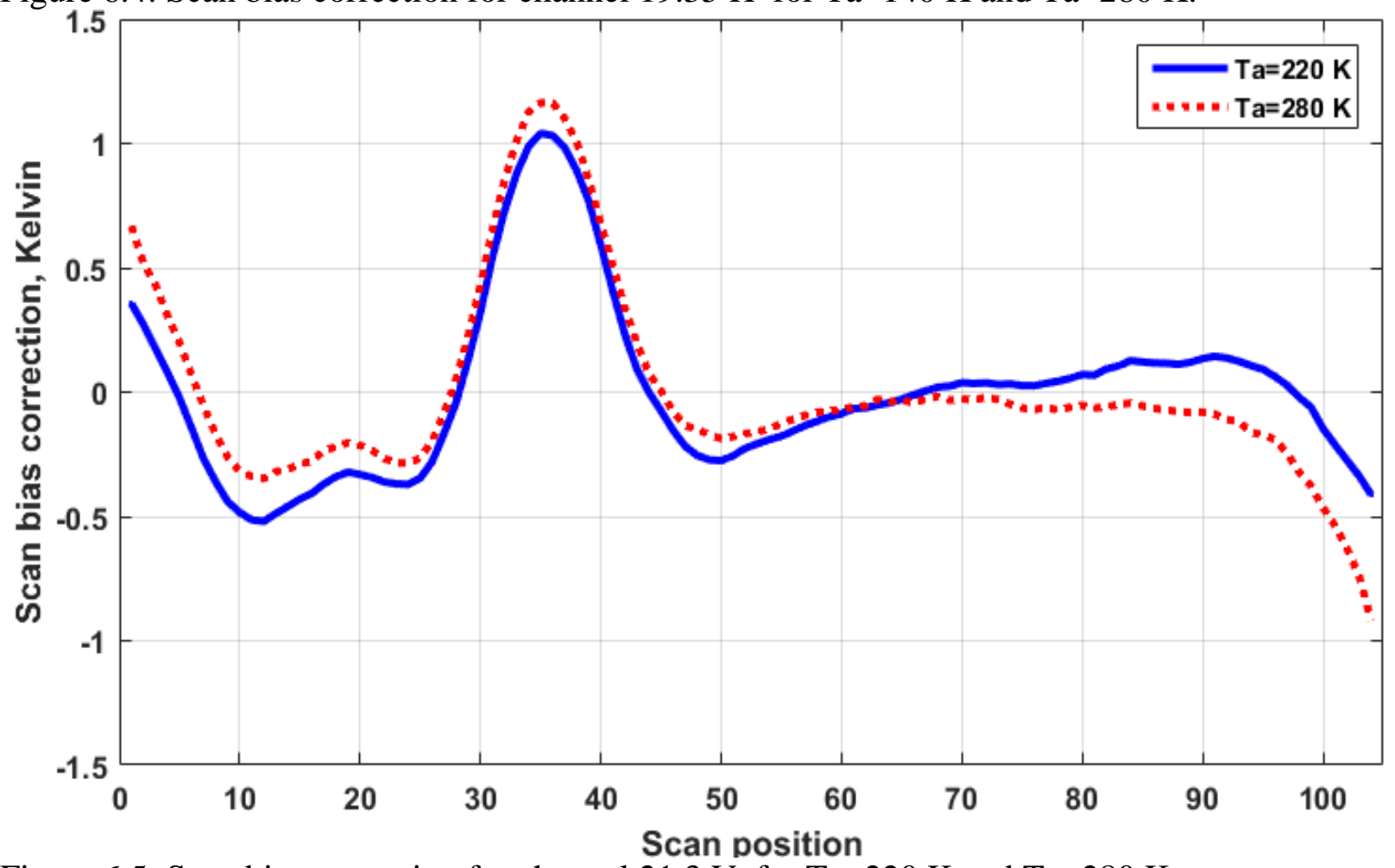

Figure 6.5: Scan bias correction for channel $21.3 \mathrm{~V}$ for $\mathrm{Ta}=220 \mathrm{~K}$ and $\mathrm{Ta}=280 \mathrm{~K}$. 


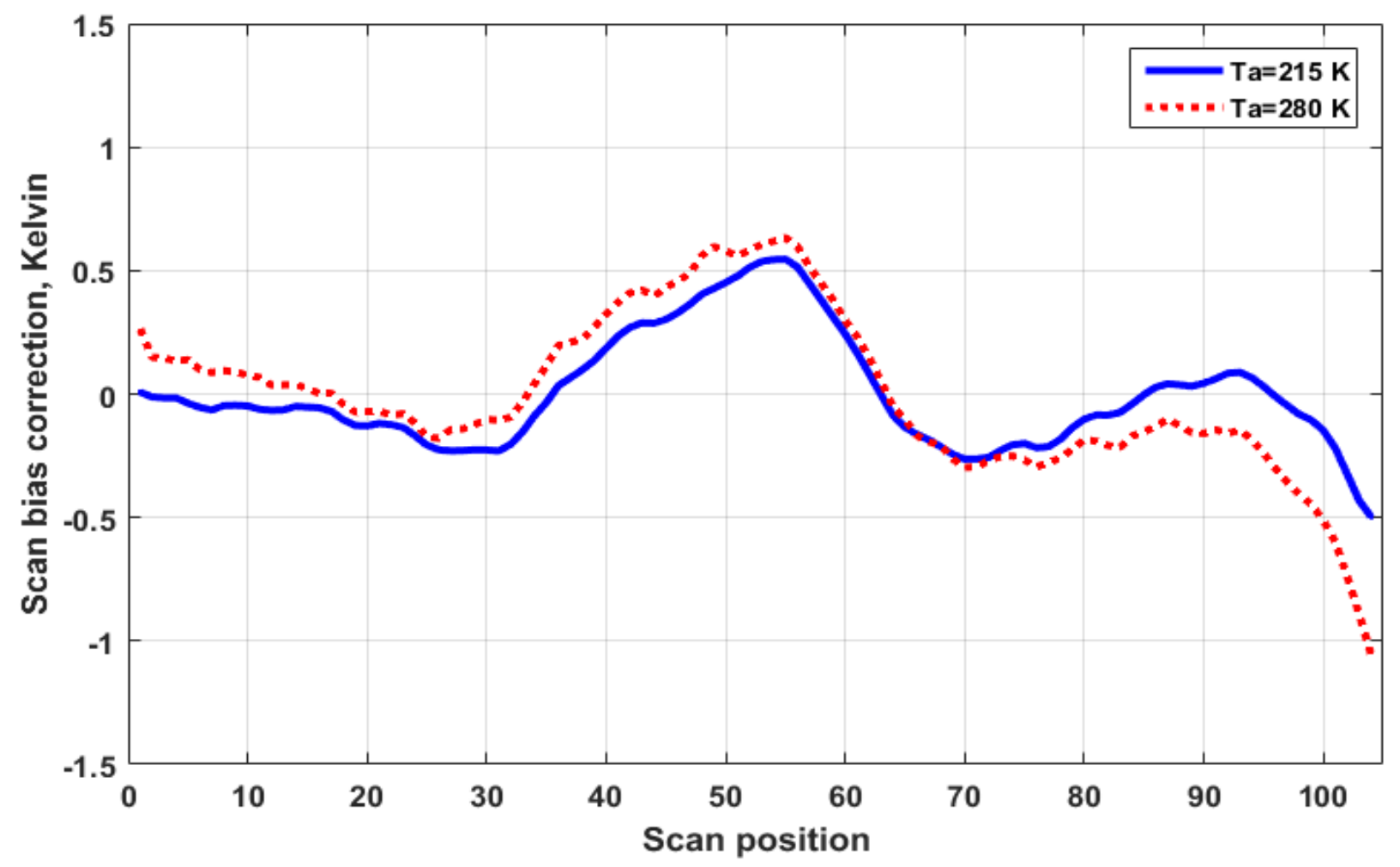

Figure 6.6: Scan bias correction for channel $37.00 \mathrm{~V}$ for $\mathrm{Ta}=215 \mathrm{~K}$ and $\mathrm{Ta}=280 \mathrm{~K}$.

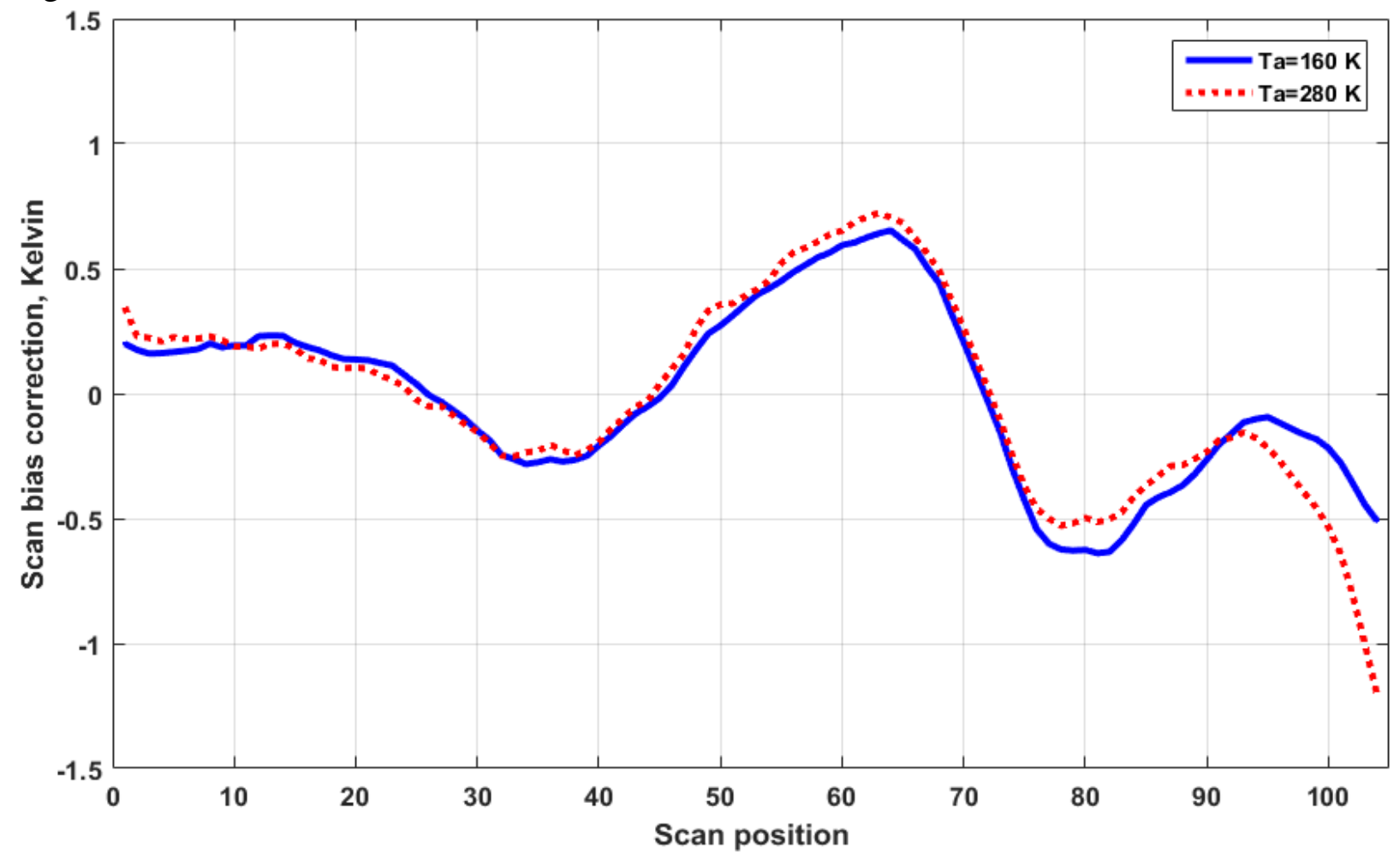

Figure 6.7: Scan bias correction for channel $37.00 \mathrm{H}$ for Ta=160 K and Ta=280 K. 


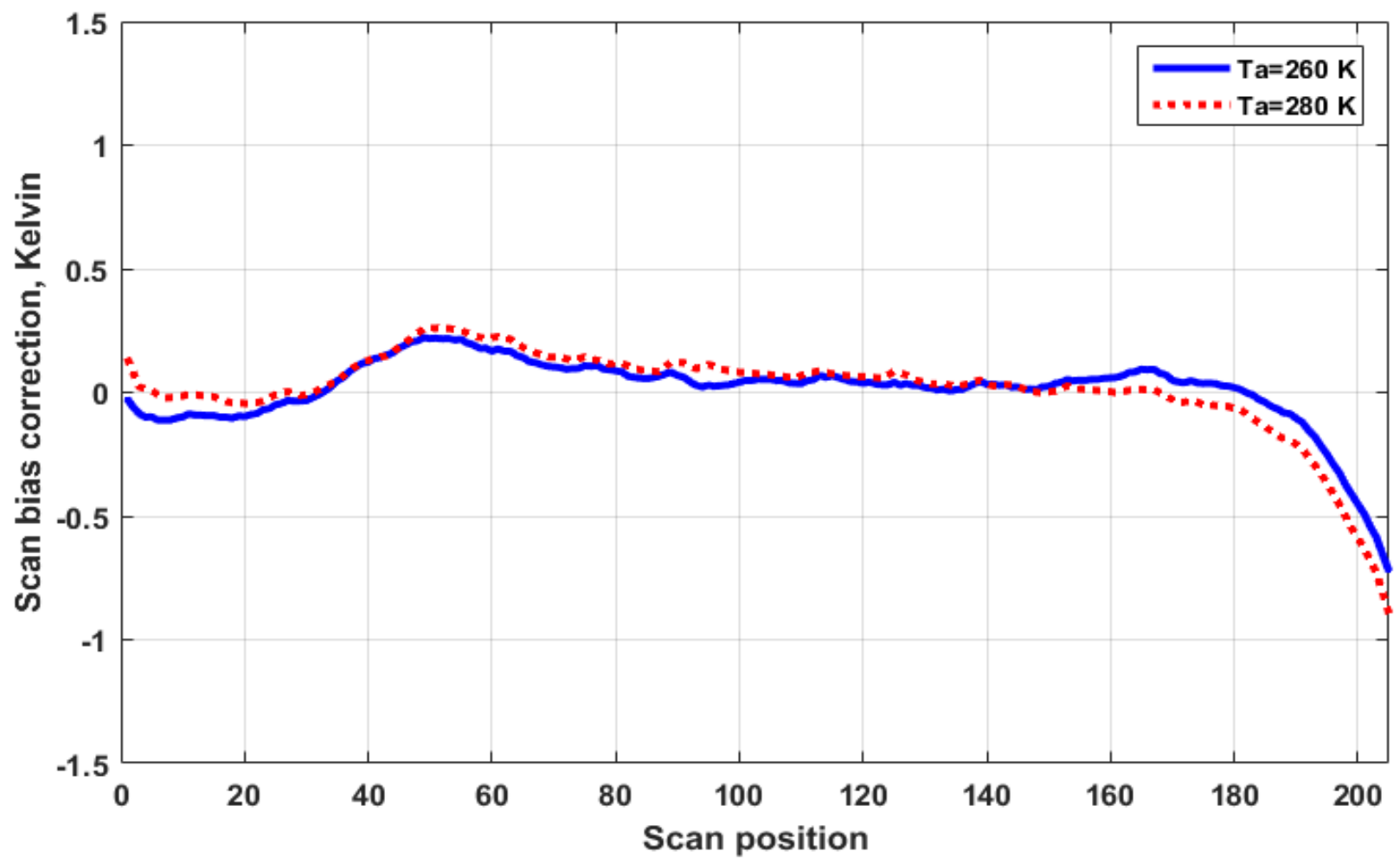

Figure 6.8: Scan bias correction for channel $85.50 \mathrm{~V}$ for $\mathrm{Ta}=260 \mathrm{~K}$ and $\mathrm{Ta}=280 \mathrm{~K}$.

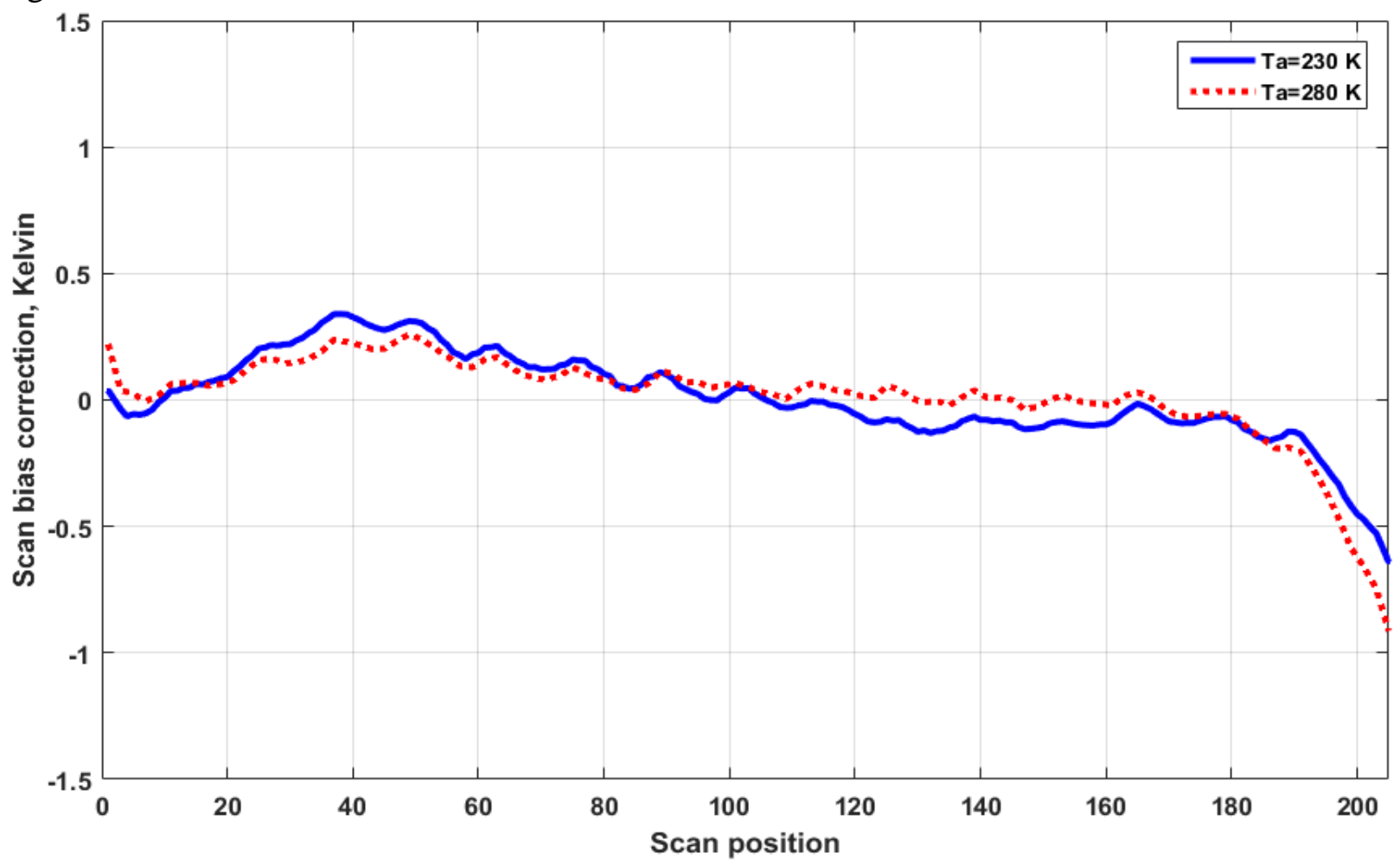

Figure 6.9: Scan bias correction for channel $85.50 \mathrm{H}$ for $\mathrm{Ta}=230 \mathrm{~K}$ and $\mathrm{Ta}=280 \mathrm{~K}$. 


\section{APPENDIX B: DERVATION OF ANTENNA PATTERN CORRECTION COEFFICIENTS}




\section{$\underline{\text { B.1 Introduction }}$}

In this appendix, the APC of the TMI 1B11 V8 will be derived in details. Also, it will be compared to the APC of the previous versions.

\section{B.2 Derivation}

The derivation of the APC coefficients for the Equation (2.12) is based on the Equations (2.8), (2.9) and (2.10), which are for removing the spill-over and cross-polarized temperatures. Equation (2.9) can redefine as

$$
T_{\text {scene }, \mathrm{V}}=\frac{T_{\text {scene }_{10.65 \mathrm{~V}}}-\chi_{10.65 \mathrm{~V}} * T_{\text {scene }, \mathrm{H}}}{\left(1-\chi_{10.65 \mathrm{~V}}\right)}
$$

Using Equation (A.1) in Equation (2.10) as

$$
\begin{aligned}
T_{\text {scene }_{10.65 H}}= & \left(1-\chi_{10.65 H}\right) * T_{\text {scene }, H}+\chi_{10.65 H} \\
& *\left(\frac{T_{\text {scene }_{10.65 V}}-\chi_{10.65 V} * T_{\text {scene }, H}}{\left(1-\chi_{10.65 V}\right)}\right)
\end{aligned}
$$

The Equation (A.2) can be rewritten as

$$
\begin{aligned}
T_{\text {scene }_{10.65 H}}= & \left(1-\chi_{10.65 H}\right) * T_{\text {scene } H} \\
& +\left(\frac{\chi_{10.65 H} * T_{\text {scene }_{10.65 V}}}{\left(1-\chi_{10.65 \mathrm{~V}}\right)}\right. \\
& \left.-\frac{\chi_{10.65 H} * \chi_{10.65 V} * T_{\text {scene }, H}}{\left(1-\chi_{10.65 V}\right)}\right)
\end{aligned}
$$

After using algebra in Equation (A.2) to be as following 


$$
\begin{aligned}
\left(\left(1-\chi_{10.65 H}\right)\right. & \left.-\frac{\chi_{10.65 H} * \chi_{10.65 V}}{\left(1-\chi_{10.65 V}\right)}\right) T_{\text {scene }, H} \\
& =T_{\text {scene }_{10.65 H}}-\left(\frac{\chi_{10.65 H} * T_{\text {scene }_{10.65 V}}}{\left(1-\chi_{10.65 V}\right)}\right)
\end{aligned}
$$

For simplicity, Equation (A.3) can be rewritten as (A.5)

$$
\begin{aligned}
& \psi_{10.65 H}=\left(\left(1-\chi_{10.65 H}\right)-\frac{\chi_{10.65 H} * \chi_{10.65 V}}{\left(1-\chi_{10.65 V}\right)}\right) \\
& T_{\text {scene }, H}=\frac{1}{\psi_{10.65 H}} T_{\text {scene }_{10.65 H}} \\
& \quad-\left(\frac{\chi_{10.65 H}}{\left(1-\chi_{10.65 V}\right) * \psi_{10.65 H}}\right) T_{\text {scene }_{10.65 V}}
\end{aligned}
$$

Finally, the APC coefficients in Equation (2.12) can be derived by using Equations (2.8) and (A.5)

$$
\begin{gathered}
C_{10.65 H}=\frac{1}{\psi_{10.65 H}\left(1-\eta_{10.65 H}\right)} \\
D_{10.65 H}=\frac{\chi_{10.65 H}}{\left(1-\chi_{10.65 V}\right) \psi_{10.65 H}\left(1-\eta_{10.65 V}\right)} \\
E_{10.65 H}=\frac{\eta_{10.65 H} * T_{c}}{\psi_{10.65 H}\left(1-\eta_{10.65 H}\right)} \\
-\frac{\chi_{10.65 H} \eta_{10.65 V} * T_{c}}{\left(1-\chi_{10.65 V}\right) \psi_{10.65 H}\left(1-\eta_{10.65 V}\right)}
\end{gathered}
$$

Where $T_{c}$ and $\eta$ are the cold space temperature and the spill-over coefficient. These equations are applied for all channels except for channel 21.3 V, which doesn't have horizontal channel. For that reason, Equation (2.11) is used to derive the APC coefficients of channel $21.3 \mathrm{~V}$ to be as following

$$
C_{21.3 V}=\frac{1}{\left(1-\eta_{21.3 V}\right) *\left(\left(1-\chi_{21.3 V}\right)+\chi_{21.3 V} * 1.6634\right)}
$$




$$
\begin{gathered}
D_{21.3 V}=0 \\
E_{21.3 V}=\frac{\eta_{21.3 V} * T_{c}}{\left(1-\eta_{21.3 V}\right)}-\chi_{21.3 V} * 195.36 \\
\left(\left(1-\chi_{21.3 V}\right)+\chi_{21.3 V} * 1.6634\right)
\end{gathered}
$$

\section{$\underline{\text { B.3 Results }}$}

There are three versions of APC coefficients during the TMI mission. The APC coefficient for version 5 and earlier are written in Table A.1. The APC coefficients of version 6 and 7 are similar, which is used the SSM/I APC coefficients as seen in Table A.2. Finally, the new APC coefficients for the new TMI version, which is the TMI 1B11 V8, is derived in this appendix (see Table A.3).

Table 6.1: TMI's APC coefficients for version 1B11 V5 and earlier

\begin{tabular}{|c|c|c|c|}
\hline $\mathbf{i}$ & $\mathbf{C}_{\mathbf{i}}$ & $\mathbf{D}_{\mathbf{i}}$ & $\mathbf{E}_{\mathbf{i}}$ \\
\hline $\mathbf{1 0 . 6 5} \mathbf{V}$ & 1.0200 & 0.0037 & 0.0163 \\
\hline $\mathbf{1 0 . 6 5} \mathbf{H}$ & 1.0209 & 0.0047 & 0.0163 \\
\hline $\mathbf{1 9 . 3 5} \mathbf{V}$ & 1.0267 & 0.0044 & 0.0223 \\
\hline $\mathbf{1 9 . 3 5} \mathbf{H}$ & 1.0277 & 0.0046 & 0.0230 \\
\hline $\mathbf{2 1 . 3 0} \mathbf{V}$ & 1.0205 & 0 & 0.0248 \\
\hline $\mathbf{3 7 . 0 0} \mathbf{V}$ & 1.0372 & 0.0246 & 0.0127 \\
\hline $\mathbf{3 7 . 0 0} \mathbf{H}$ & 1.0317 & 0.0192 & 0.0124 \\
\hline $\mathbf{8 5 . 5 0} \mathbf{V}$ & 1.0331 & 0.0208 & 0.0123 \\
\hline $\mathbf{8 5 . 5 0} \mathbf{H}$ & 1.0412 & 0.0304 & 0.0109 \\
\hline
\end{tabular}


Table 6.2: TMI's APC coefficients for version 1B11 V6\&7

\begin{tabular}{|c|c|c|c|}
\hline $\mathbf{i}$ & $\mathbf{C}_{\mathbf{i}}$ & $\mathbf{D}_{\mathbf{i}}$ & $\mathbf{E}_{\mathbf{i}}$ \\
\hline $\mathbf{1 0 . 6 5} \mathbf{~ V}$ & 1.02001 & 0.00375 & 0.0163 \\
\hline $\mathbf{1 0 . 6 5} \mathbf{~ H}$ & 1.02094 & 0.00468 & 0.04439 \\
\hline $\mathbf{1 9 . 3 5} \mathbf{V}$ & 1.03698 & 0.00394 & 0.06083 \\
\hline $\mathbf{1 9 . 3 5} \mathbf{~ H}$ & 1.0277 & 0.00544 & 0.06285 \\
\hline $\mathbf{2 1 . 3 0} \mathbf{~ V}$ & 1.02151 & 0 & -1.05520 \\
\hline $\mathbf{3 7 . 0 0} \mathbf{~ V}$ & 1.03681 & 0.02226 & 0.03457 \\
\hline $\mathbf{3 7 . 0 0} \mathbf{~ H}$ & 1.04217 & 0.02762 & 0.03399 \\
\hline $\mathbf{8 5 . 5 0} \mathbf{V}$ & 1.02632 & 0.01432 & 0.03351 \\
\hline $\mathbf{8 5 . 5 0} \mathbf{~ H}$ & 1.03219 & 0.02019 & 0.02969 \\
\hline
\end{tabular}

Table 6.3: TMI's APC coefficients for version 1B11 V8

\begin{tabular}{|c|c|c|c|}
\hline $\mathbf{i}$ & $\mathbf{C}_{\mathbf{i}}$ & $\mathbf{D}_{\mathbf{i}}$ & $\mathbf{E}_{\mathbf{i}}$ \\
\hline $\mathbf{1 0 . 6 5} \mathbf{V}$ & 1.02002 & 0.00376 & 0.04439 \\
\hline $\mathbf{1 0 . 6 5} \mathbf{H}$ & 1.02096 & 0.00470 & 0.04439 \\
\hline $\mathbf{1 9 . 3 5} \mathbf{V}$ & 1.02673 & 0.00445 & 0.06083 \\
\hline $\mathbf{1 9 . 3 5} \mathbf{H}$ & 1.02768 & 0.00466 & 0.06285 \\
\hline $\mathbf{2 1 . 3 0} \mathbf{V}$ & 1.02100 & 0 & -1.05520 \\
\hline $\mathbf{3 7 . 0 0} \mathbf{V}$ & 1.03788 & 0.02522 & 0.03457 \\
\hline $\mathbf{3 7 . 0 0} \mathbf{H}$ & 1.03208 & 0.01963 & 0.03399 \\
\hline $\mathbf{8 5 . 5 0} \mathbf{V}$ & 1.03358 & 0.02130 & 0.03351 \\
\hline $\mathbf{8 5 . 5 0} \mathbf{H}$ & 1.04212 & 0.03124 & 0.02969 \\
\hline
\end{tabular}




\section{APPENDIX C: THE STATUS OF -Y SOLAR ARRAY}


The purpose of this appendix is to document the articulation of the "-Y solar array" over the 17-year lifetime of the TMI. This information was provided by Mr. Steve Bilano of the NASA Goddard Space Flight Center.

\section{C.1 TRMM Weakly Reports}

DOY 147 - 153

02-150 (Thursday, May 30 ${ }^{\text {th }}$ )

A Solar Array feathering test was performed for two orbits between, 15:04:51z and 18:22:50z (CR \#615, and \#616). The Solar Array tracking was changed to +/- 1 degree from +/50 degrees. The Power subsystem was configured for Constant Current Mode 3 (24 Amps per battery) from Constant Current Mode 2 (12 Amps per battery) during the test. The test was successful, and another test is scheduled for 02-156 (Thursday, June $6^{\text {th }}$ ).

June 3, 2002 - June 9, 2002

DOY 154 - 160

02-156 (Wednesday, June $5^{\text {th }}$ )

A Solar Array feathering test was performed for two orbits between, 15:04:51z and 18:22:50z (CR \#620, and \#621). The Solar Array tracking was changed to +/- 1 degree from +/50 degrees. The Power subsystem was configured for Constant Current Mode 3 (24 Amps per battery) and VT6 from Constant Current Mode 2 (12 Amps per battery) and VT5 during the test. The test was successful, and another test is scheduled for 02-162 (Tuesday, June $10^{\text {th }}$ ). 
June 10, 2002 - June 16, 2002

DOY $161-167$

The Beta angle range for $02-168$ to $02-174$ is $-9.1^{\circ}$ to $11.8^{\circ}$.

02-162 (Tuesday, June $11^{\text {th }}$ )

A Solar Array feathering test was performed between, 12:16:26z and 18:37:13z (CR \#624 and \#625). The Solar Array tracking was changed to +/- 1 degree from +/- 50 degrees. The Power subsystem was configured for Constant Current Mode 3 (24 Amps per battery) and VT6 from Constant Current Mode 2 (12 Amps per battery) and VT5 during the test. The test was successful, and additional testing is planned in the near future.

June 17, 2002 - June 23, 2002

DOY $168-174$

The Beta angle range for $02-175$ to $02-181$ is $11.8^{\circ}$ to $37.3^{\circ}$. Another Solar Array Feathering test is scheduled from $02-175$ to $02-179$ (June $24^{\text {th }}$ to June $29^{\text {th }}$ ).

June 24, 2002 - June 30, 2002

DOY $175-181$

The Beta angle range for $02-182$ to $02-188$ is $37.3^{\circ}$ to $57.4^{\circ}$. Another Solar Array Feathering test began on $02-175$ (June $24^{\text {th }}$ ), and will continue to approx. July $20^{\text {th }}$.

July 1, 2002 - July 7, 2002 
DOY $182-188$

Beta angle range for $02-189$ to $02-195$ is $57.4^{\circ}$ to $40.5^{\circ}$. Solar Array Feathering test continues. It was started on 02-175 (June $\left.24^{\text {th }}\right)$, and will continue to approx. July $20^{\text {th }}$.

And next week noted:

Solar Array Feathering test continues. It was started on 02-175 (June $24^{\text {th }}$ ), and will continue to July $19^{\text {th }}$ (before the Yaw Maneuver).

02-200 (Friday, July 19 ${ }^{\text {th }}$ )

The Solar Arrays were returned to tracking mode with $\pm 50^{\circ}$ stops, VT mode 5, and 12 amps per battery following Delta-V \#403, and all subsystems performed nominally. The next feathering test will occur in August due to solar beta angle.

$2^{\text {nd }}$ extended feathering test reports noted in weekly:

August 5, 2002 - August 11, 2002

DOY $217-223$

The Beta angle range for $02-224$ to $02-230$ is $12.13^{\circ}$ to $38.76^{\circ}$ The Solar Array

Feathering test (Solar Array stops limited to $+/-1^{\circ}$ ) will continue on 02-224 (August $12^{\text {th }}$ ) when the Beta Angle reaches $+15^{\circ}$.

August 12, 2002 - August 18, 2002

DOY $224-230$ 
The Beta angle range for $02-231$ to $02-237$ is $38.78^{\circ}$ to $43.96^{\circ}$. The Solar Array Feathering test resumed on 02-224 (August $12^{\text {th }}$ ) when the Beta Angle reached $+15^{\circ}$, and will continue to approximately September $2^{\text {nd }}$

September 2, 2002 - September 8, 2002

DOY 245 - 251

Beta angle range for $02-252$ to $02-258$ is $-12.3^{\circ}$ to $-31.9^{\circ}$.

The Solar Array Feathering test ended on 02-247 (Wednesday, September $4^{\text {th }}$ ).

A sample of how the exact orbits for feathering can be checked from ATT plots.

Looking at $2^{\text {nd }}$ special test, noted as follows

A Solar Array feathering test was performed for two orbits between, 15:04:51z and 18:22:50z (CR \#620, and \#621). The Solar Array tracking was changed to +/- 1 degree from $+/-$ 50 degrees.

\section{C.2 Attitude Analysis}

This is the attitude analysis that provided by Stephen Bilanow to detect the specific orbit for these solar array changes. Looking at the next four figures which are for orbits 25976 to 25979 , we see the small spacecraft motions in roll and yaw from the motion of the solar arrays to the feathered position during orbit night. These occur after shadow entry and before sunrise in orbits 25976, and 25979, but not in 25977 and 25978. At the end of 25978 plot we see a disturbance but that belongs to the next orbit. PR data is shown in these plots only within orbit span and there is otherwise a 4-minute overlap in the gyro-propagated attitudes which are plotted. 
With $+\mathrm{X}$ forward (Yaw 0) the Sun Sensor 1 data shown in red near 0 occurs just after sunrise, and Sun Sensor 2 which is biased -.2 degrees (and not used currently) is picked up just before Sunset. The interferences in the hot load, and the array shadowing, occur just before sunset. 
TRMM Version 004 Attitude, Pitch Estimate

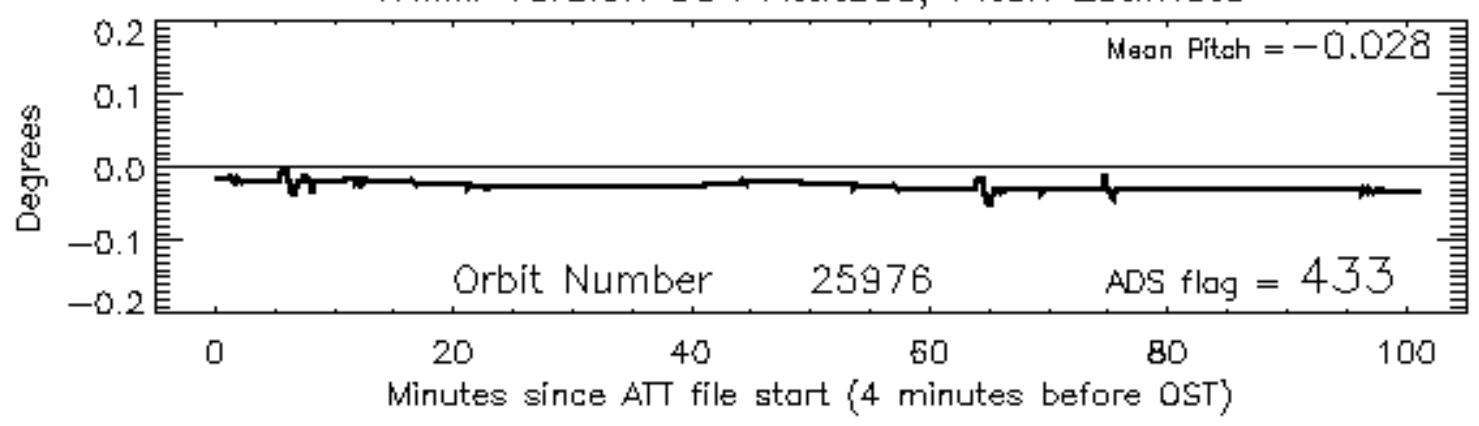

Roll Estimate, and PR Roll Measurements

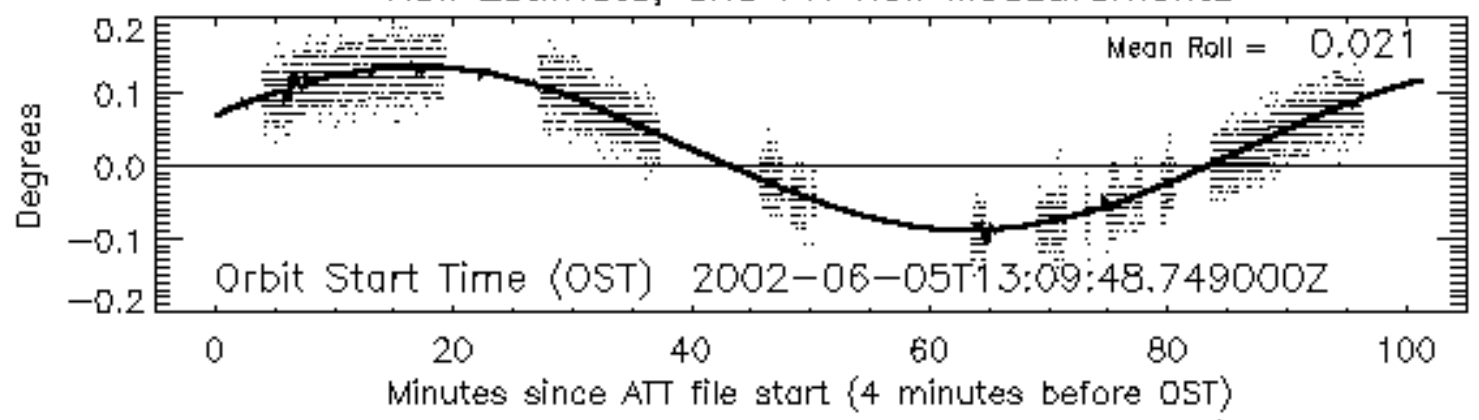

TRMk ve attltude, run 2016-12-01, p4f, regeneration of Version o04 plote at cleser $+/-0.2$ scale

Yaw Estimate
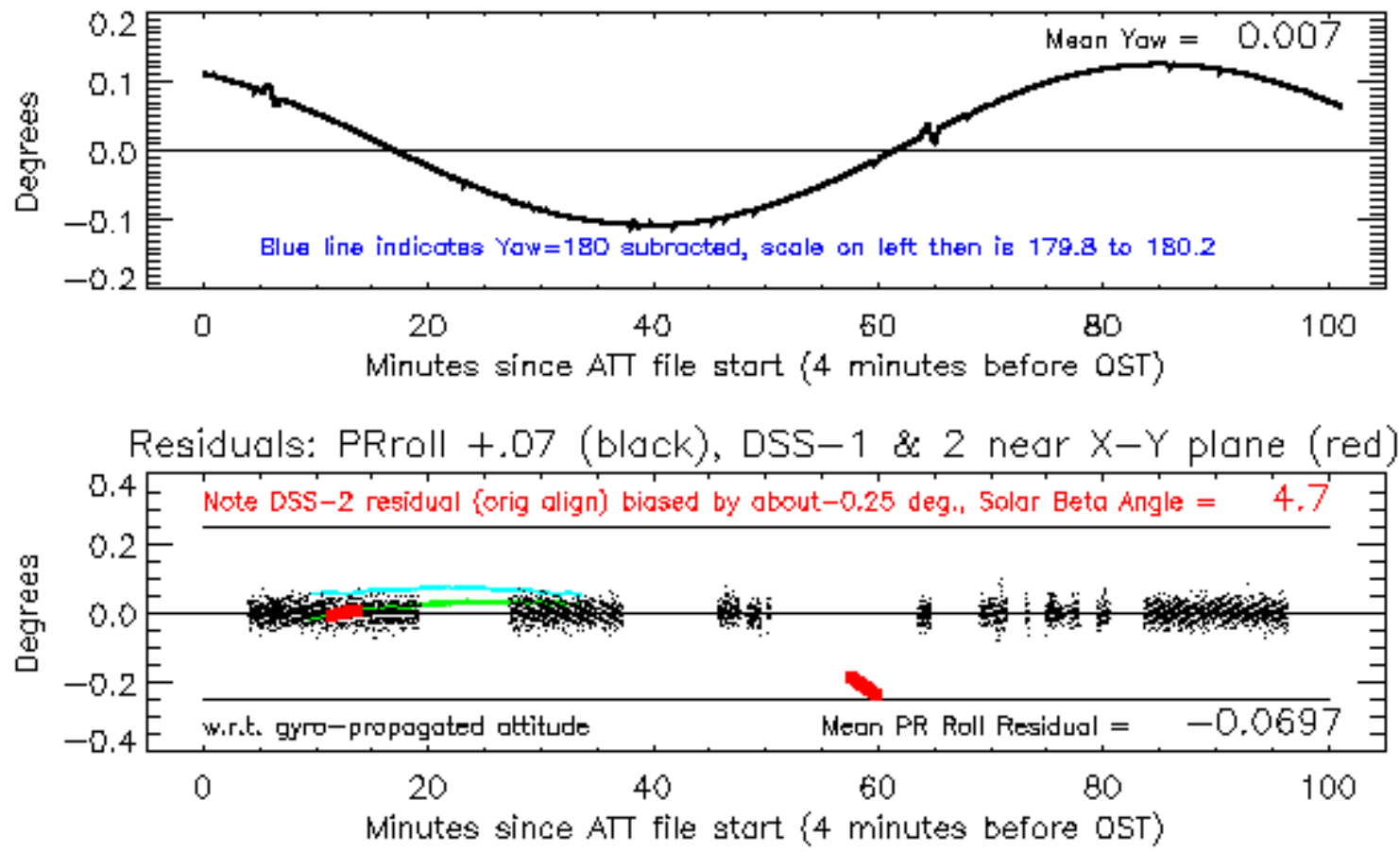

Figure 6.10: Attitude analysis shows the non-feathered solar array of orbit 25976 


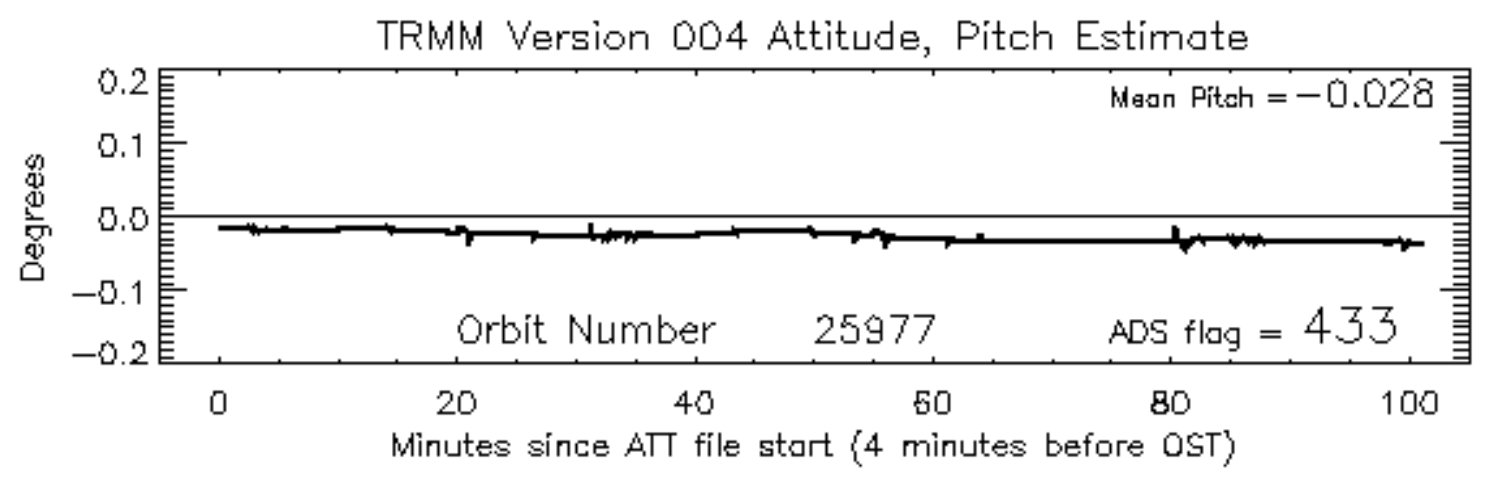

Roll Estimate, and PR Roll Measurements

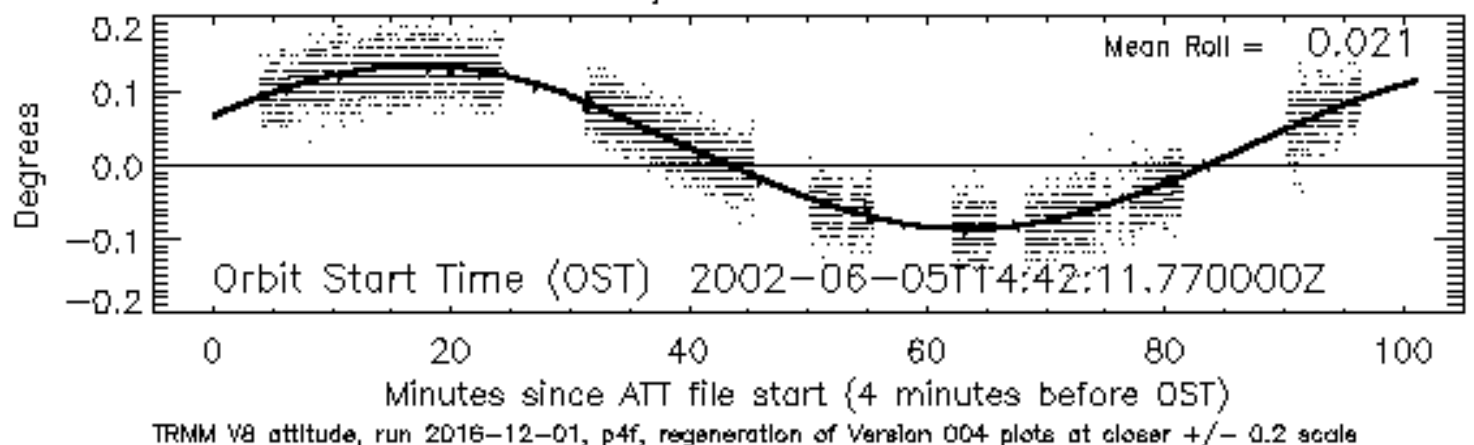

Yaw Estimate
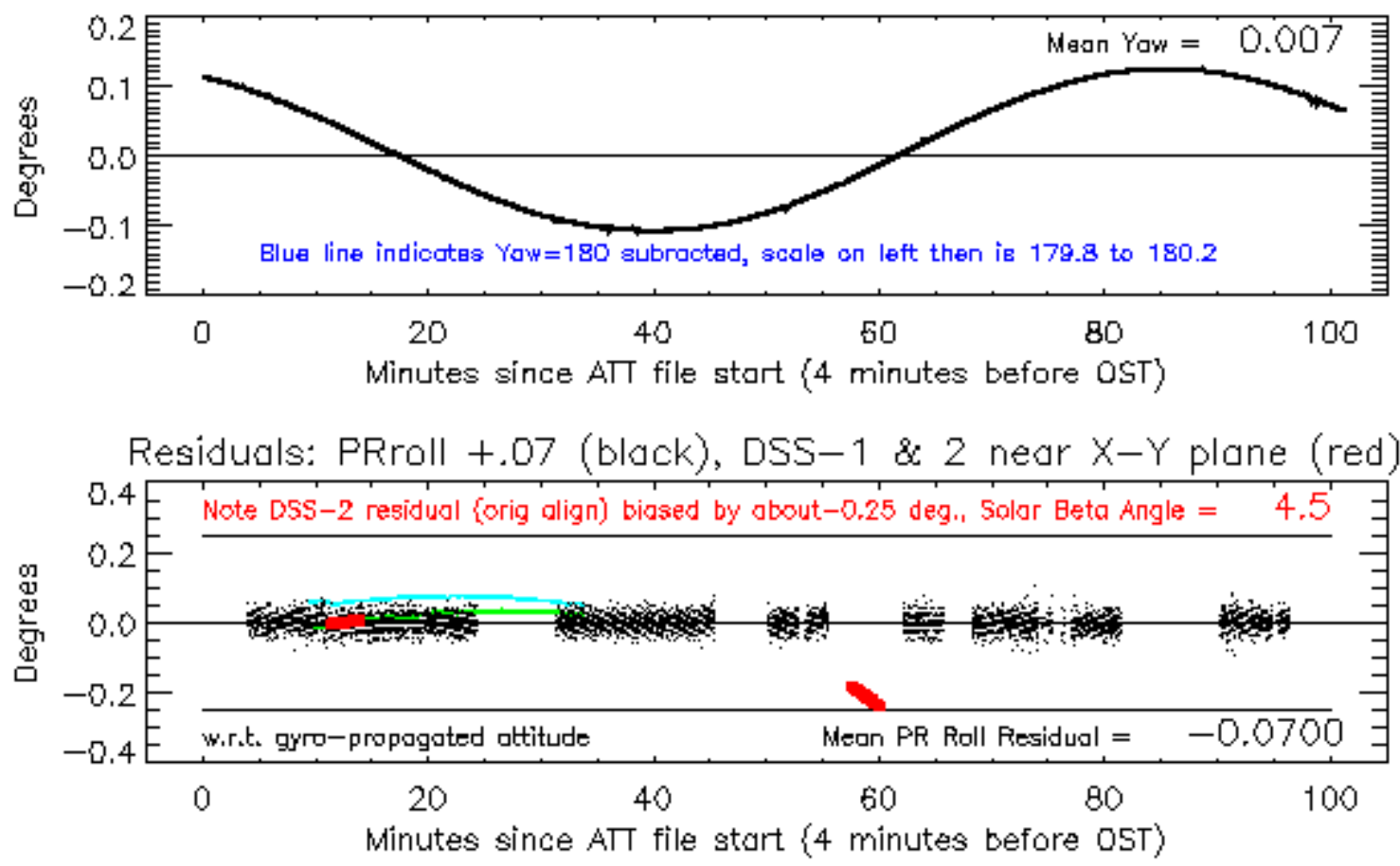

Figure 6.11: Attitude analysis shows the feathered solar array of orbit 25977. 
TRMM Version 004 Attitude, Pitch Estimate

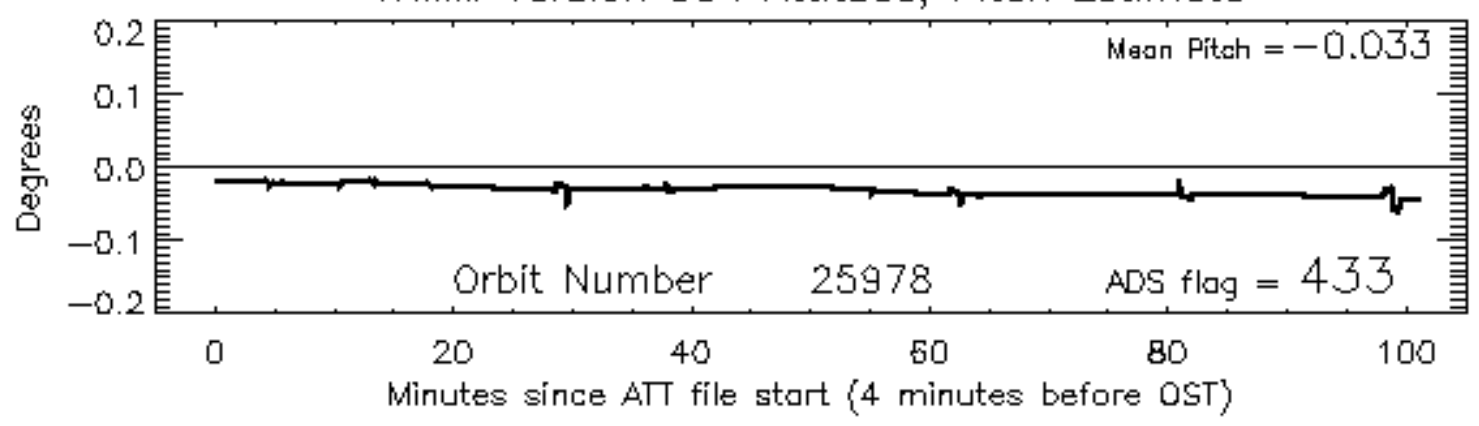

Roll Estimate, and PR Roll Measurements

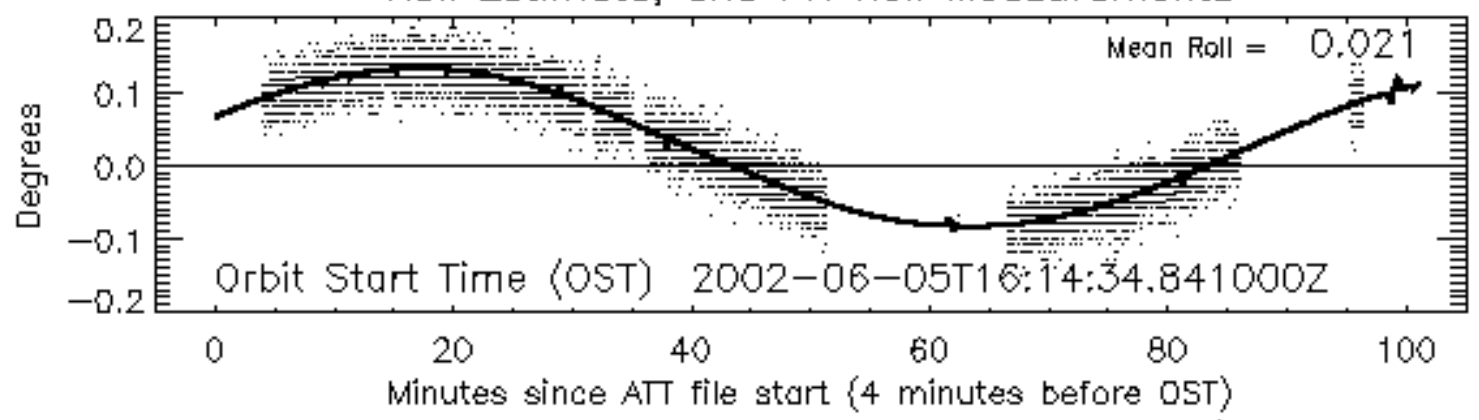

TRMk ve attltude, run 2016-12-01, p4f, regeneration of Version o04 plote at cleser $+/-0.2$ scale

Yaw Estimate
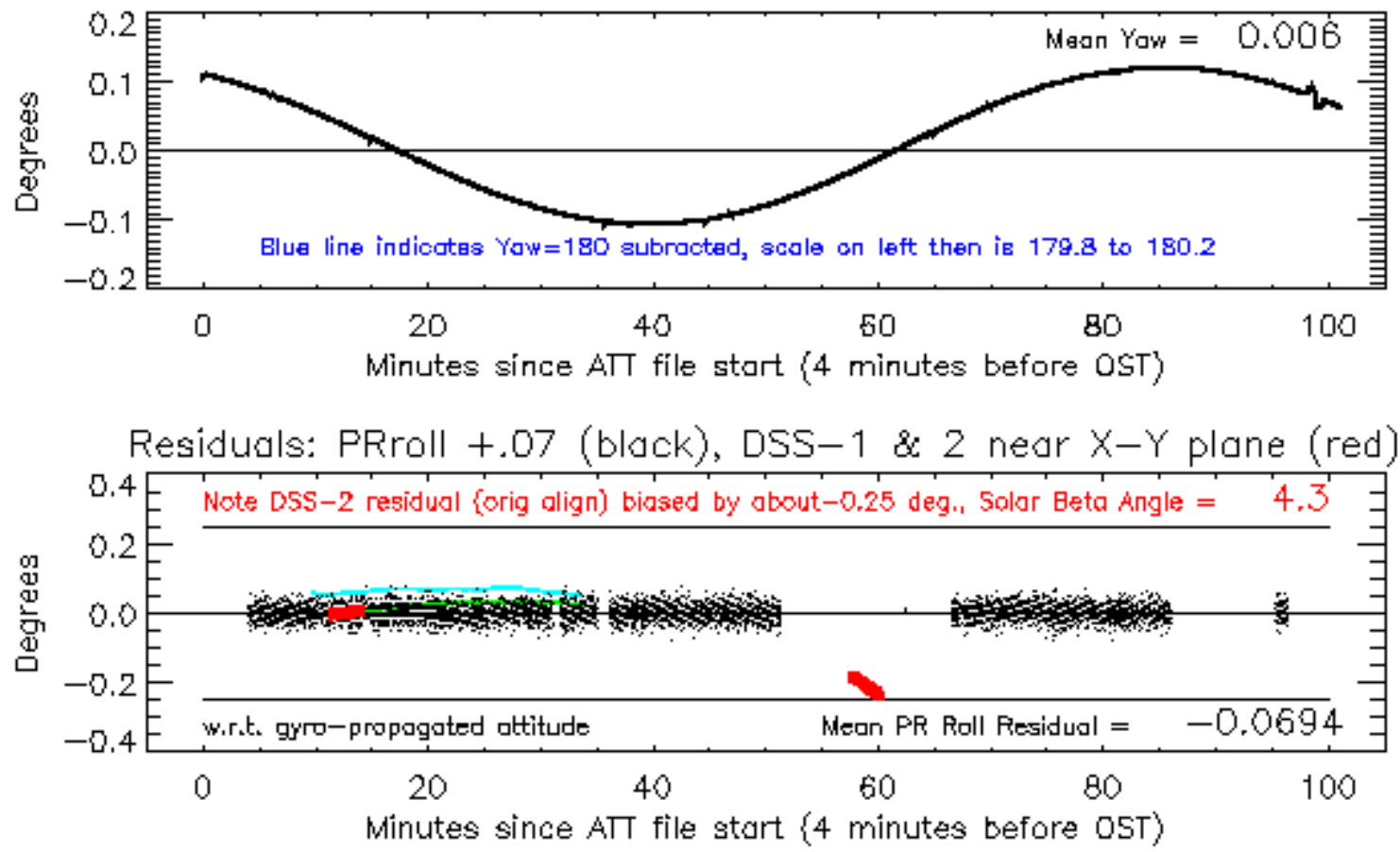

Figure 6.12: Attitude analysis shows the feathered solar array of orbit 25978. 
TRMM Version 004 Attitude, Pitch Estimate

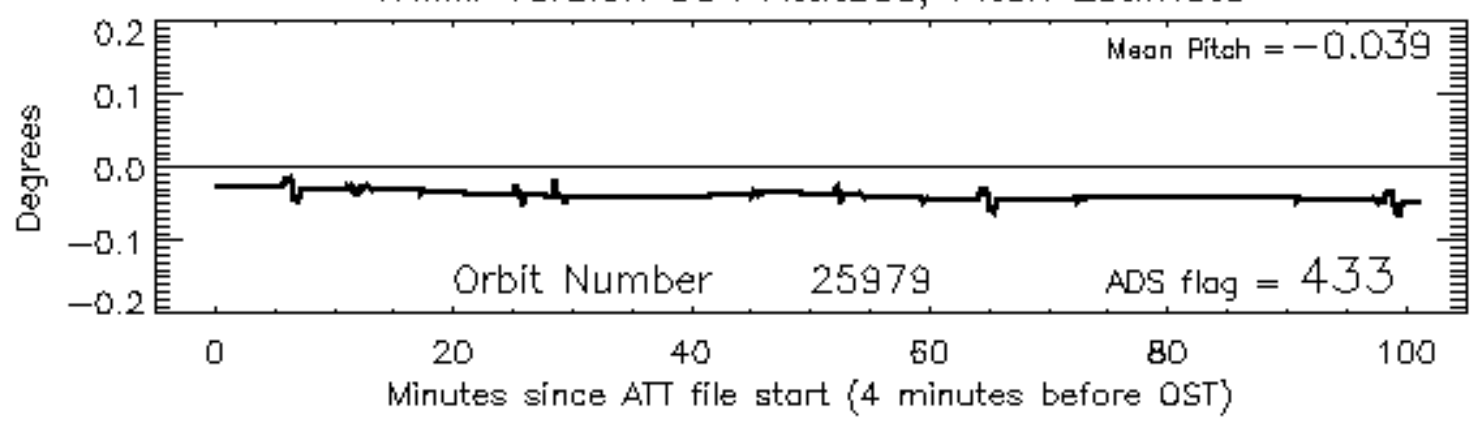

Roll Estimate, and PR Roll Measurements

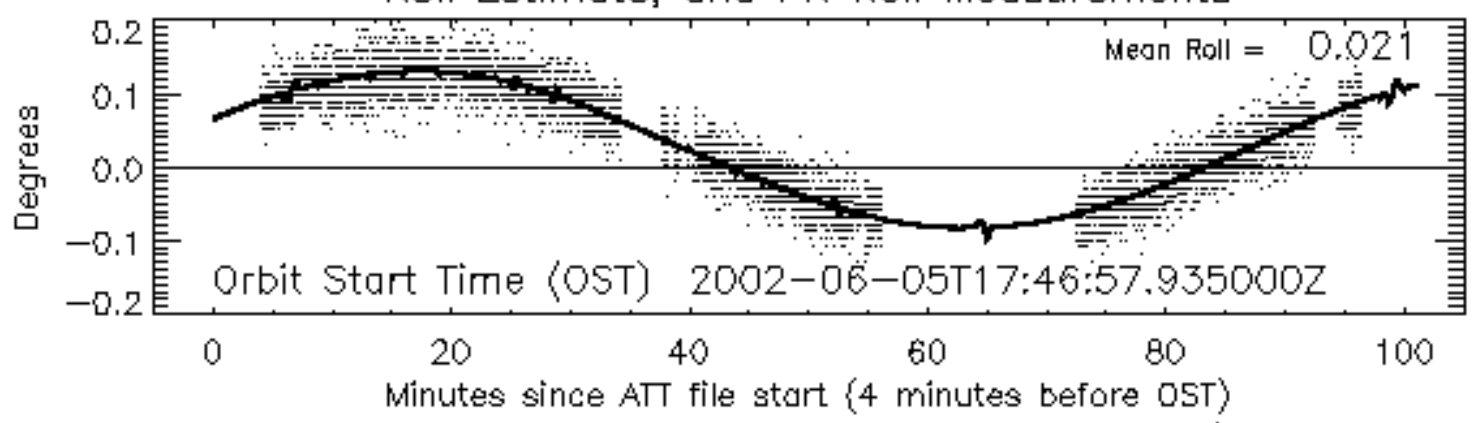

TRMk ve attltude, run 2016-12-01, p4f, regeneration of Version o04 plote at cleser $+/-0.2$ scale

Yaw Estimate
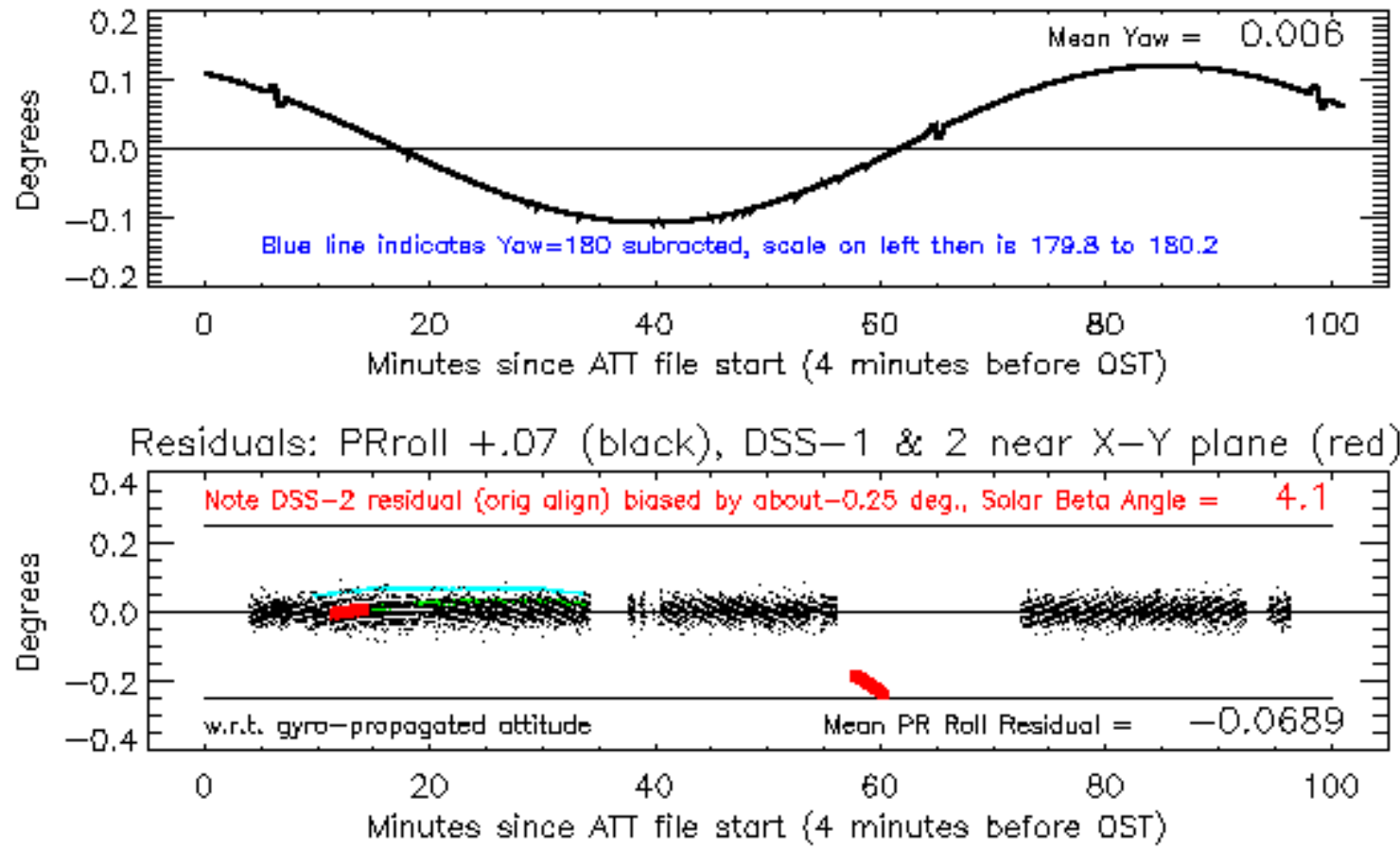

Figure 6.13: Attitude analysis shows the non-feathered solar array of orbit 25979 


\section{$\underline{\text { C.3 Email }}$}

This was an email from Stephen Bilanow regarding the status of the - Y solar array.

From: Stephen Bilanow

To: XCAL group

Date: Fri, Feb 17, 2017 at 1:38 AM

"Xcalers,

The following notes are relevant to tomorrows discussion of period two noted in UCF slide number 2. It turns out the division of solar array operating periods is a bit more complex than we'd thought.

With some assistance from Crag Jamieson and the helpdesk records we were able to find some dates for feathering tests that were done to the array tracking before the -Y (sun side) array was frozen at 0 degrees for feathering.

We found 3 short tests for few orbits in 2002 on May 30, June 5, and then June 11. And then there were some extended tests (both at yaw=0):

June 24 to July 19

and

\section{August 12 to September 4}

These last two explain the data in Faisal's slide 16 on the right. Note, these were implemented with a slight difference from the final frozen 0 degree feathered configuration for $-Y$. The array stops were set at $+/-1$ degree. For most of the pre-boost period the arrays were stopped at $+/-50$ 
degrees. Also both arrays were feathered for this test. It was only after the anomaly investigation, starting September 5th 2002, that they worked out having the $+Y$ array track on its own. For some period after September 5th the array stop limits were also set to $+/-1$ degree. The final configuration with $-\mathrm{Y}$ parked at 0 and $+\mathrm{Y}$ nominal tracking was implemented on October 11, 2002. For our purposes the array stops at $+/-1$ just means a 1 degree extra rotation to the array during the shadowing. This is a relatively small change for shadowing of the TMI hot load. However there was an early period of more extended tracking. So, let me just quote some key information from the MOC final report:

"In 1998, the FOT observed that when the Beta Angle was near maximum, the -Y array actuator temperature peaked around $41.5^{\circ} \mathrm{C}$. This occurred because the angle of the array allowed sunlight to warm the SADA. This was a concern because the Penzane lubricant could degrade and/or evaporate at higher than normal bearing temperatures. ... In order to reduce the duty cycle of the array, the FOT uplinked an updated ACS Table \#76 that limited the array to track $\pm 50^{\circ}$ (instead of the designed $\pm 130^{\circ}$.) This also required a patch ... and Additional updates to FDCs $\ldots$

After some more digging and document review late, I found a reference to dates for the $+/-130$ to $+/-50$ tracking changes in the notes for

CCR 025 submitted 05/08/1998

Problem Description: Due to high temperatures on the -Y Solar Array actuator, the deployables engineer wishes to decrease the motion on the Solar Array as much as possible. Also, keeping the arrays closer to "feathered" decreases drag and increases mission life. 
Proposed Solution: Change the S/A Software Stops from +/- 130 degrees to +/- 50 degress via an ACS table change. Since the table change alone did not solve the problem (commands were still being sent to the solar arrays after the software stops were reached), a patch to the ACS FSW is needed

\section{Closed - Completed 12/15/1998}

So it is apparent there is yet another period with a different operating mode for the arrays at the very beginning of the mission, and probably some different interference patterns for sunshine on the hot load. More array shadowing may have reduced the interference in the hot load in the very early mission.

For most of the pre-boost period I think we are seeing the shadow of the array parked at the 50 degree position, which lasts from sunrise until the sun is 50 degrees from orbit noon. From that point in the orbit to around 50 degrees past local noon, the array tracks the sun for maximum power. The array is stopped at the 50 degrees offset (plus or minus), and held there through the sunset span. At night the arrays were feathered to reduce drag. At Yaw zero, it is the sunset span where the array shadows the sunshine into the hot load. At Yaw 180, it is around sunrise that the array shadowing pattern is seen.

Just to make things a little more complicated, I just found a whole bunch of notes about other special feathering tests in a list of command requests, such as 7/1/2002 Change SA T Thresholds to 20 degrees for SA Feathering. So, will have to investigate if other tests are worthy of concern. 
I know this is challenging to visualize, let alone keep track of the different changes, but we can discuss this information at the telecon. I had not been aware of these details of the array tracking changes previously.

regards,

Steve" 


\section{APPENDIX D: HOT LOAD CORRECTION RESULTS FOR ALL CHANNELS AND ALL PERIODS}



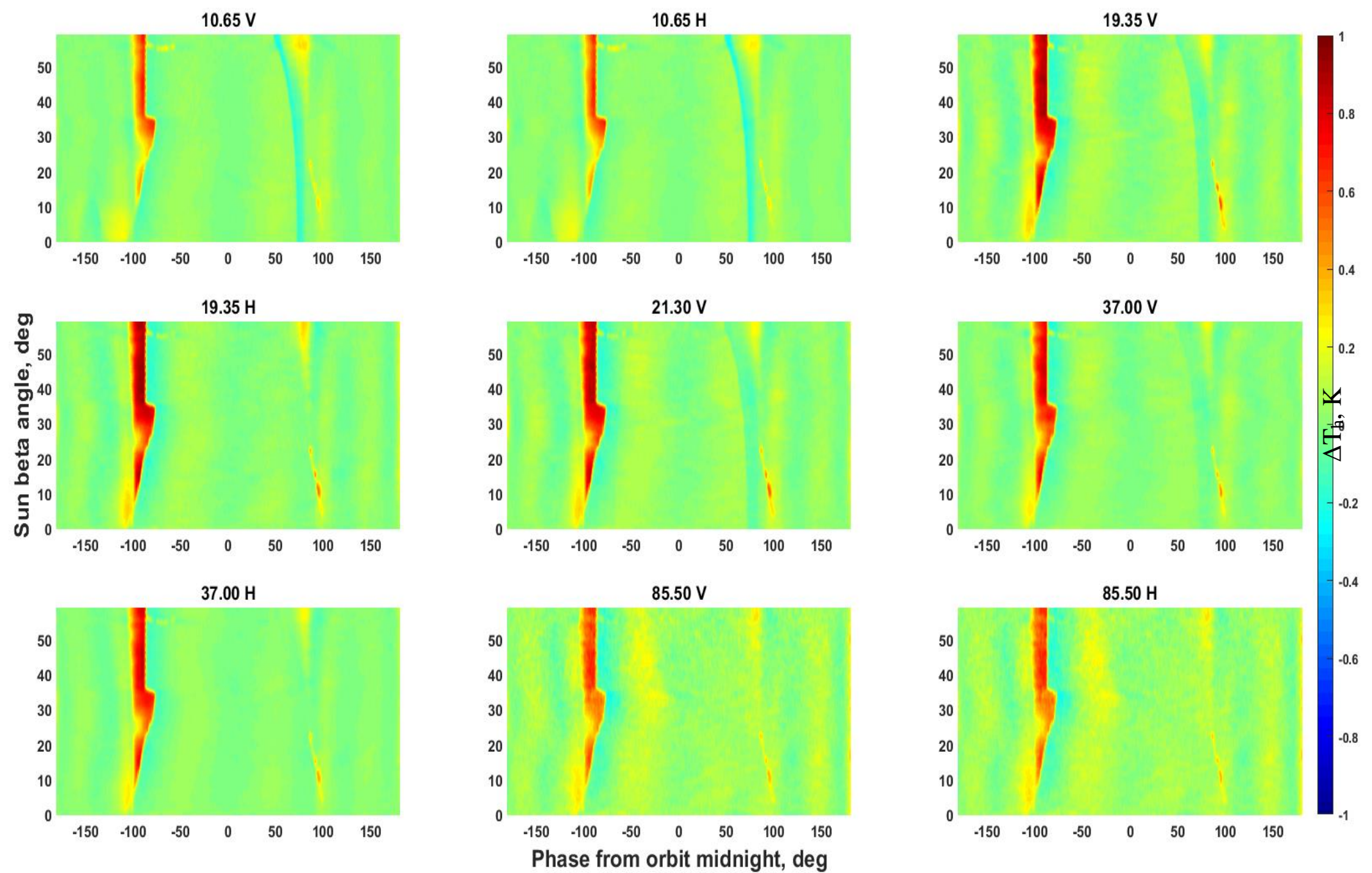

Figure 6.14: Hot load correction for all channels (yaw0, period 1) 

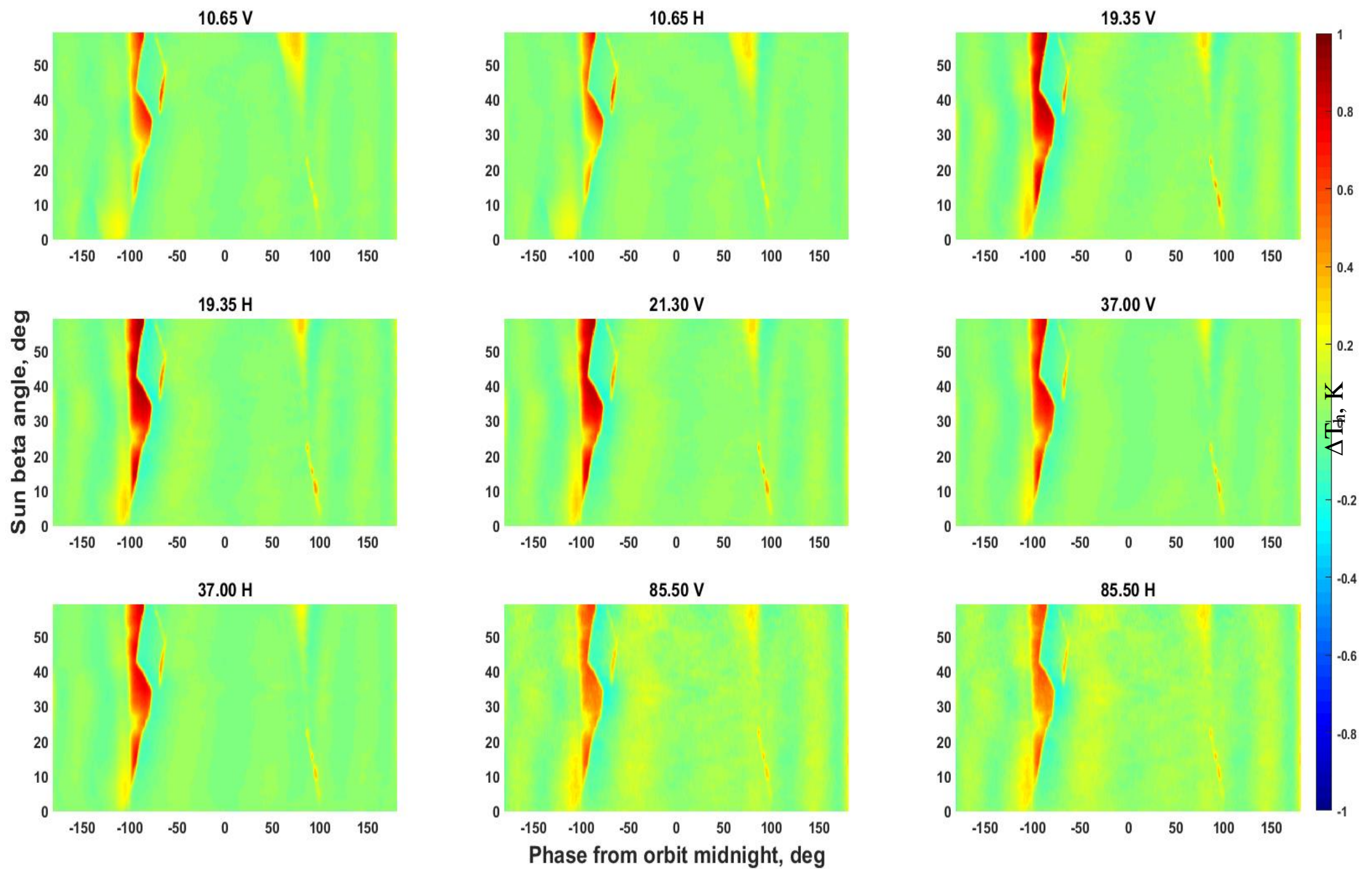

Figure 6.15: Hot load correction for all channels (yaw0, period 2) 

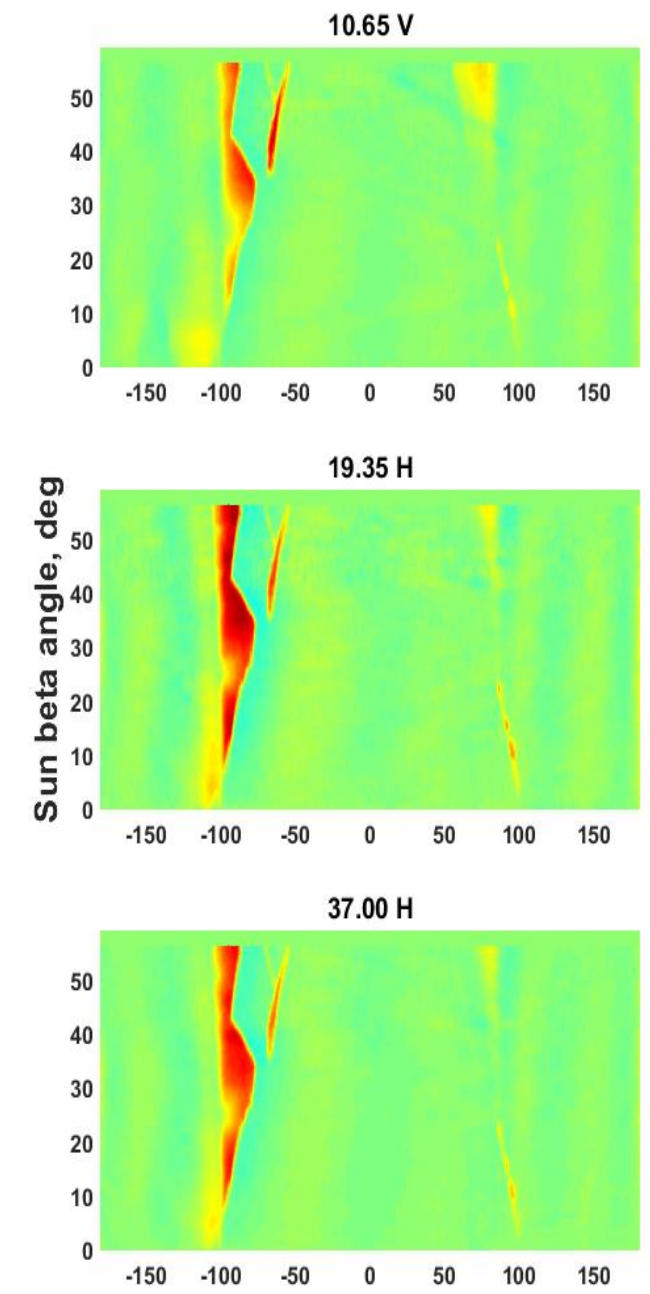
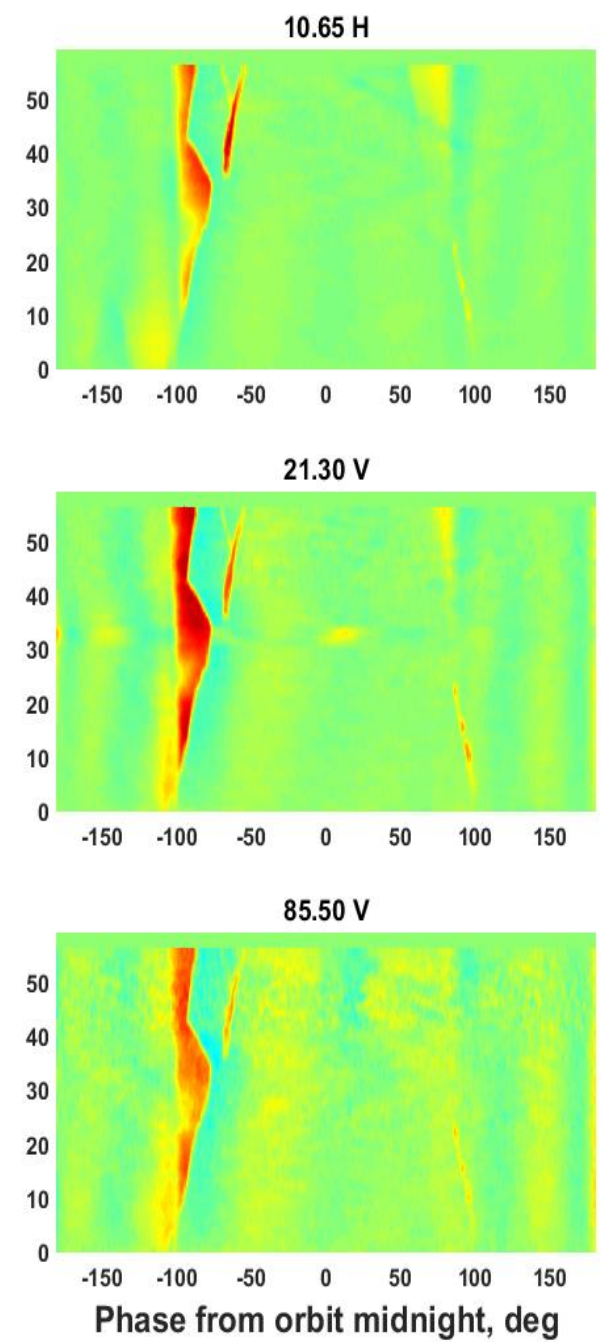
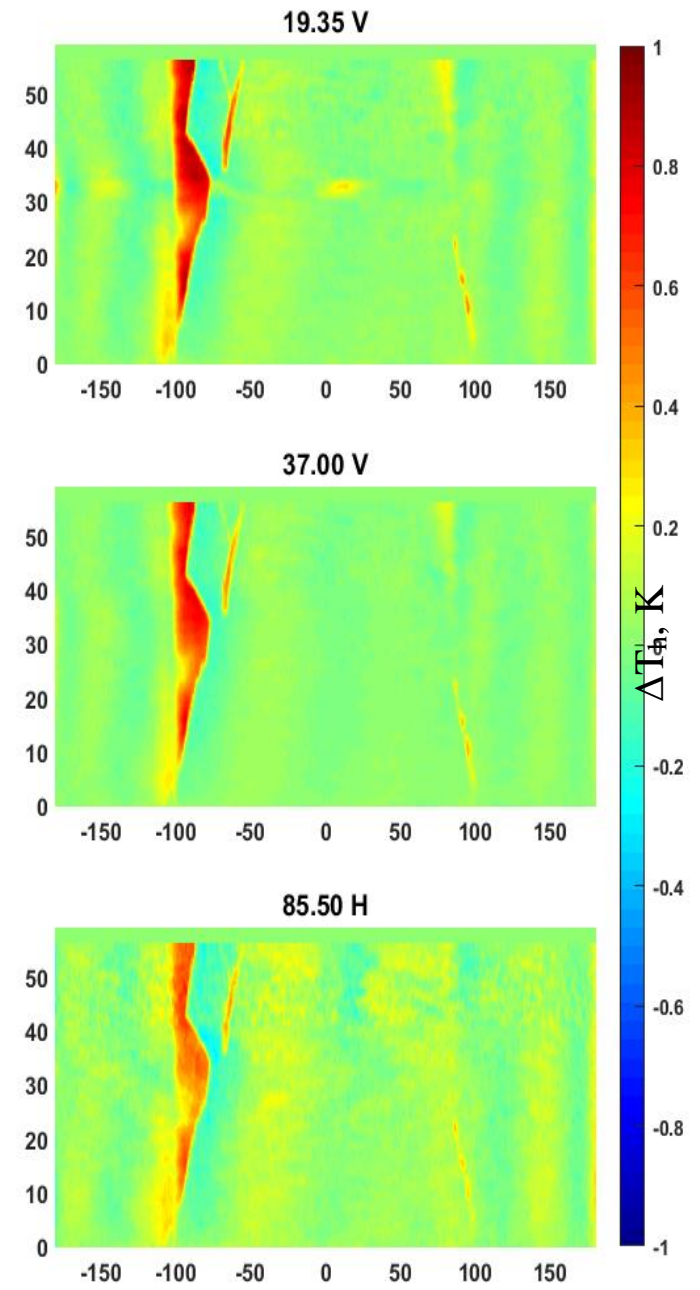

Figure 6.16: Hot load correction for all channels (yaw0, period 3) 

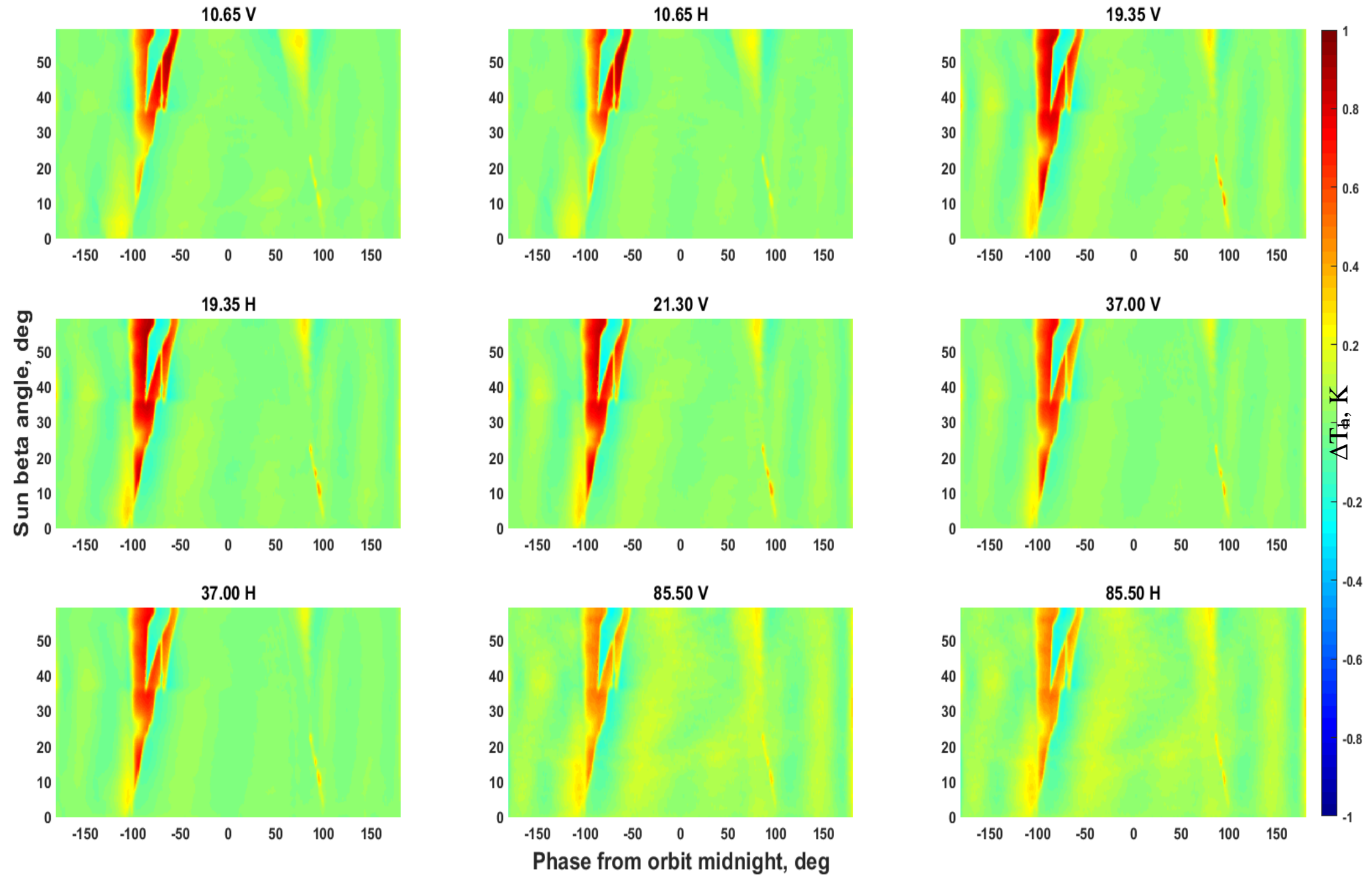

Figure 6.17: Hot load correction for all channels (yaw0, period 4) 

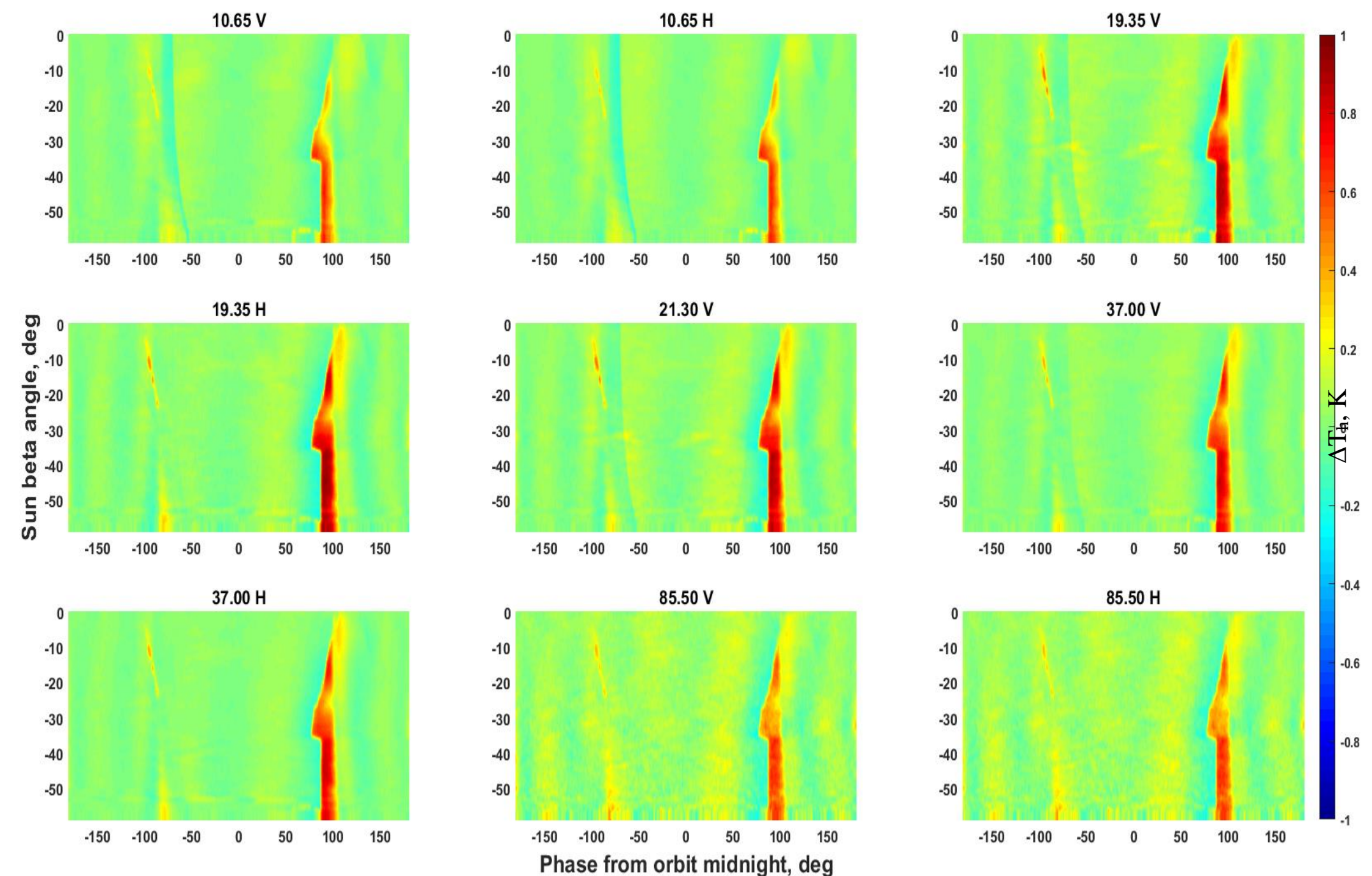

Figure 6.18: Hot load correction for all channels (yaw180, period 1) 

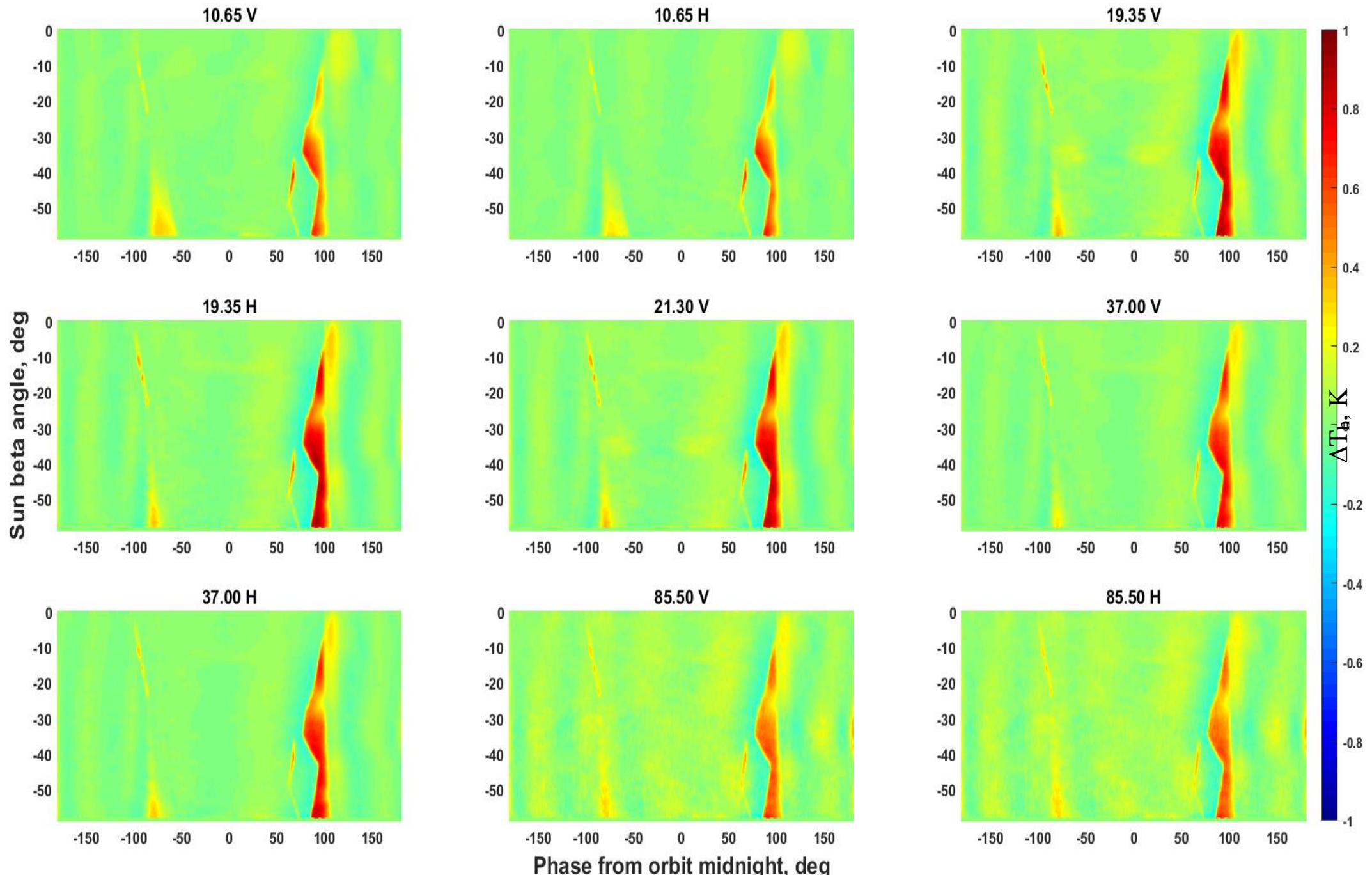

Figure 6.19: Hot load correction for all channels (yaw180, period 2) 

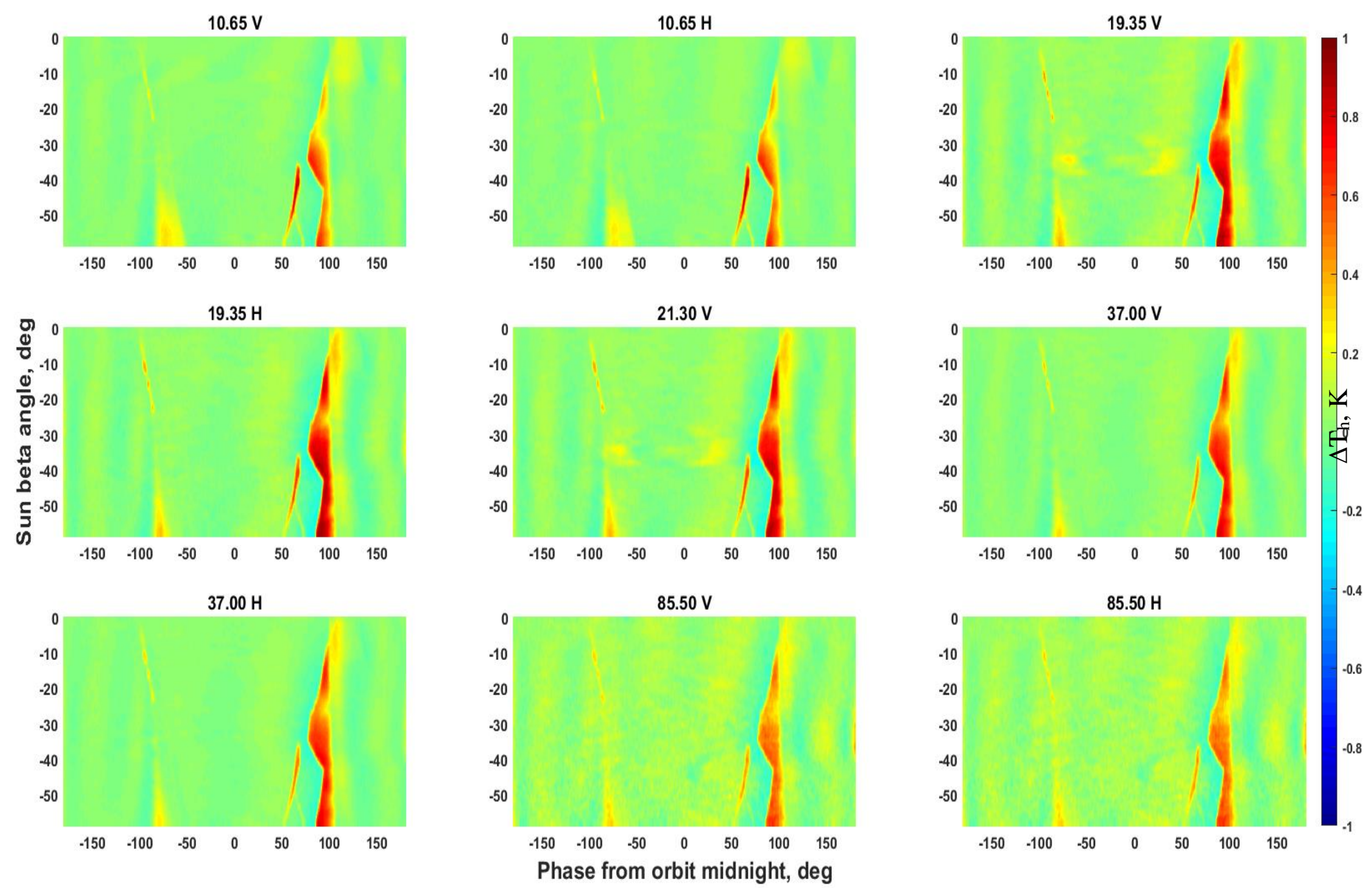

Figure 6.20: Hot load correction for all channels (yaw180, period 3) 

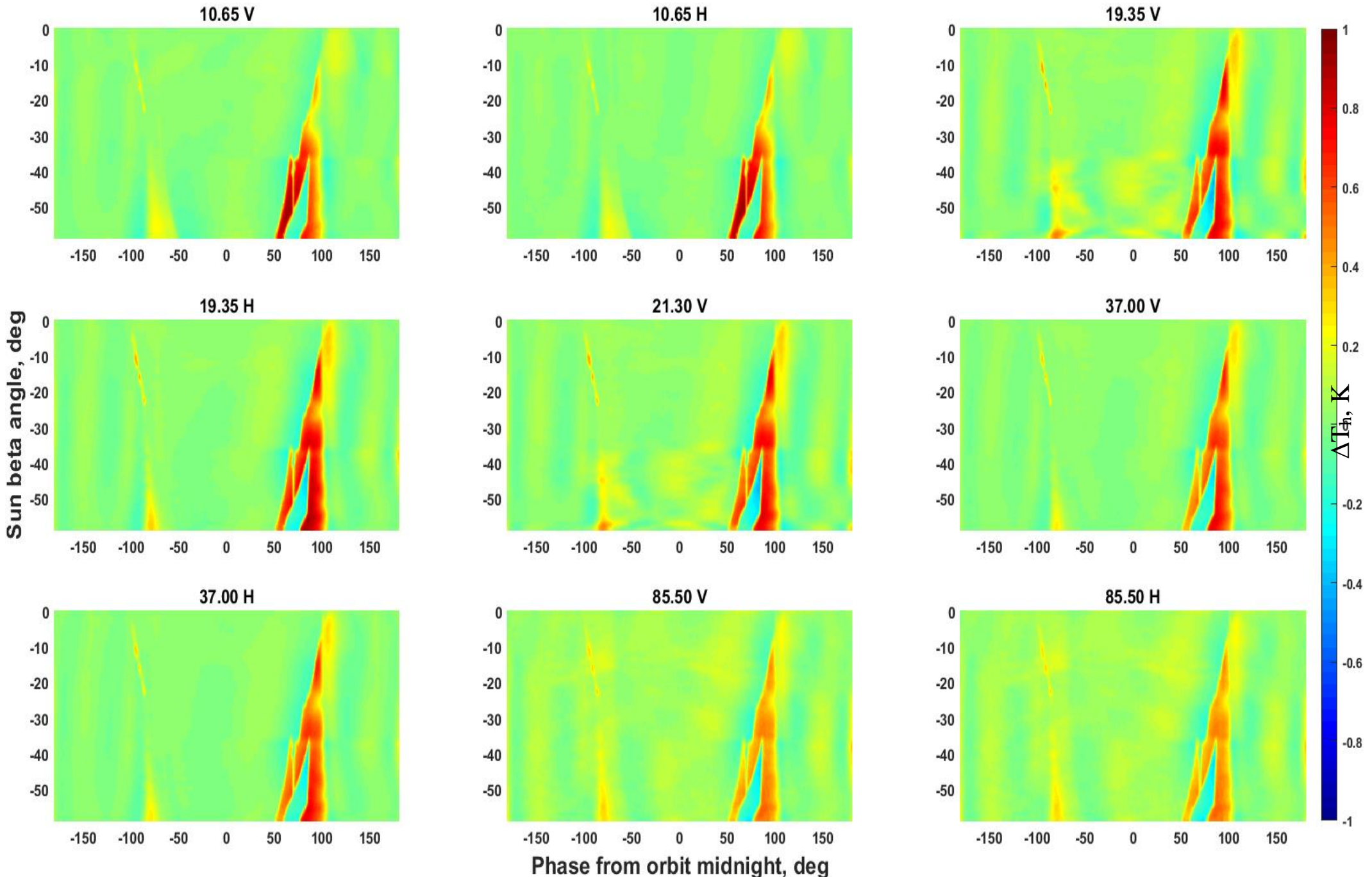

Figure 6.21: Hot load correction for all channels (yaw180, period 4) 


\section{APPENDIX E: PHYSICAL TEMPERATURE OF THE MAIN}

REFLECTOR AT YAW 180 


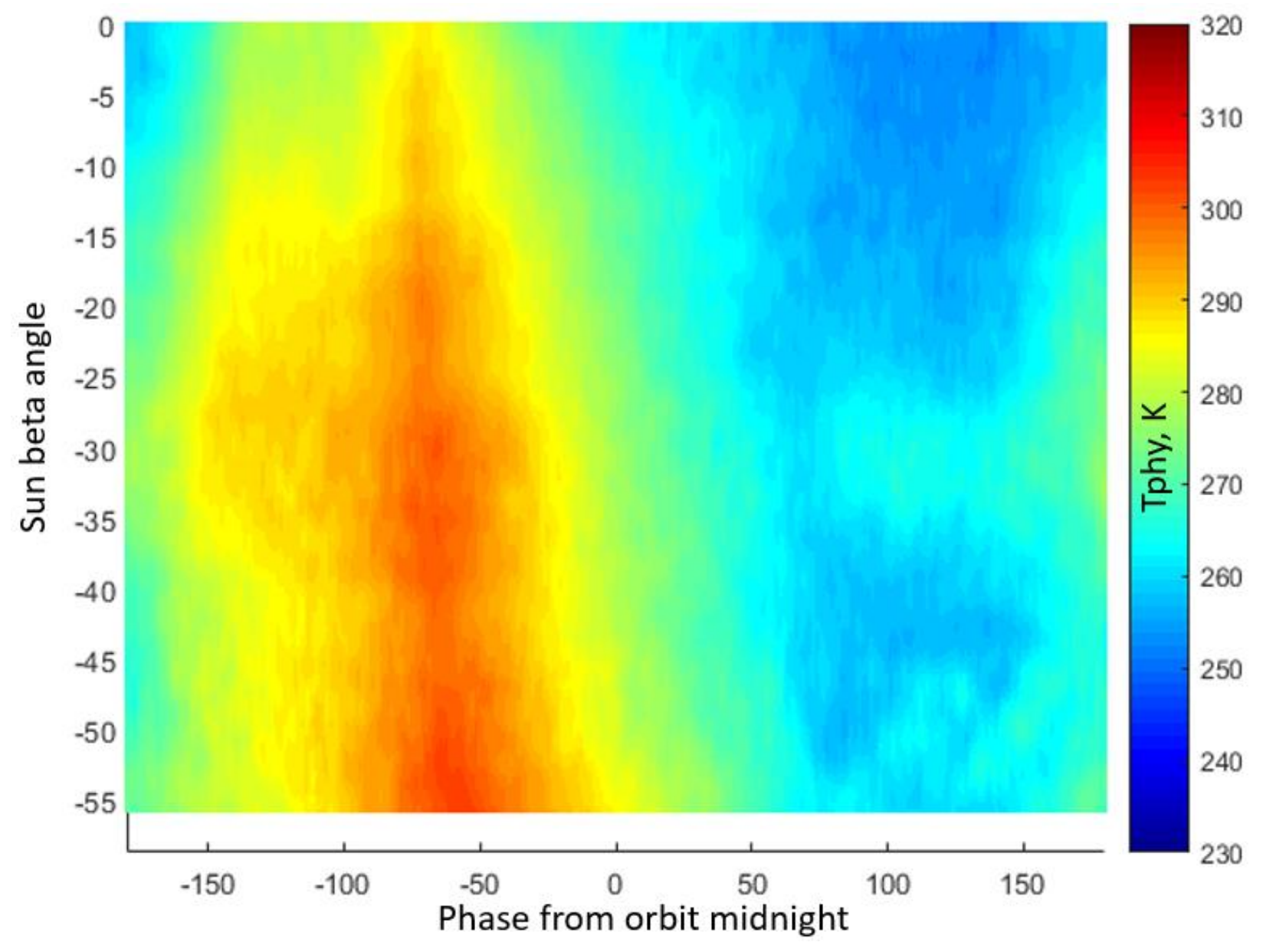

Figure 6.22: 2D matrix of MR physical temperature (yaw 180 and pre-boost). 


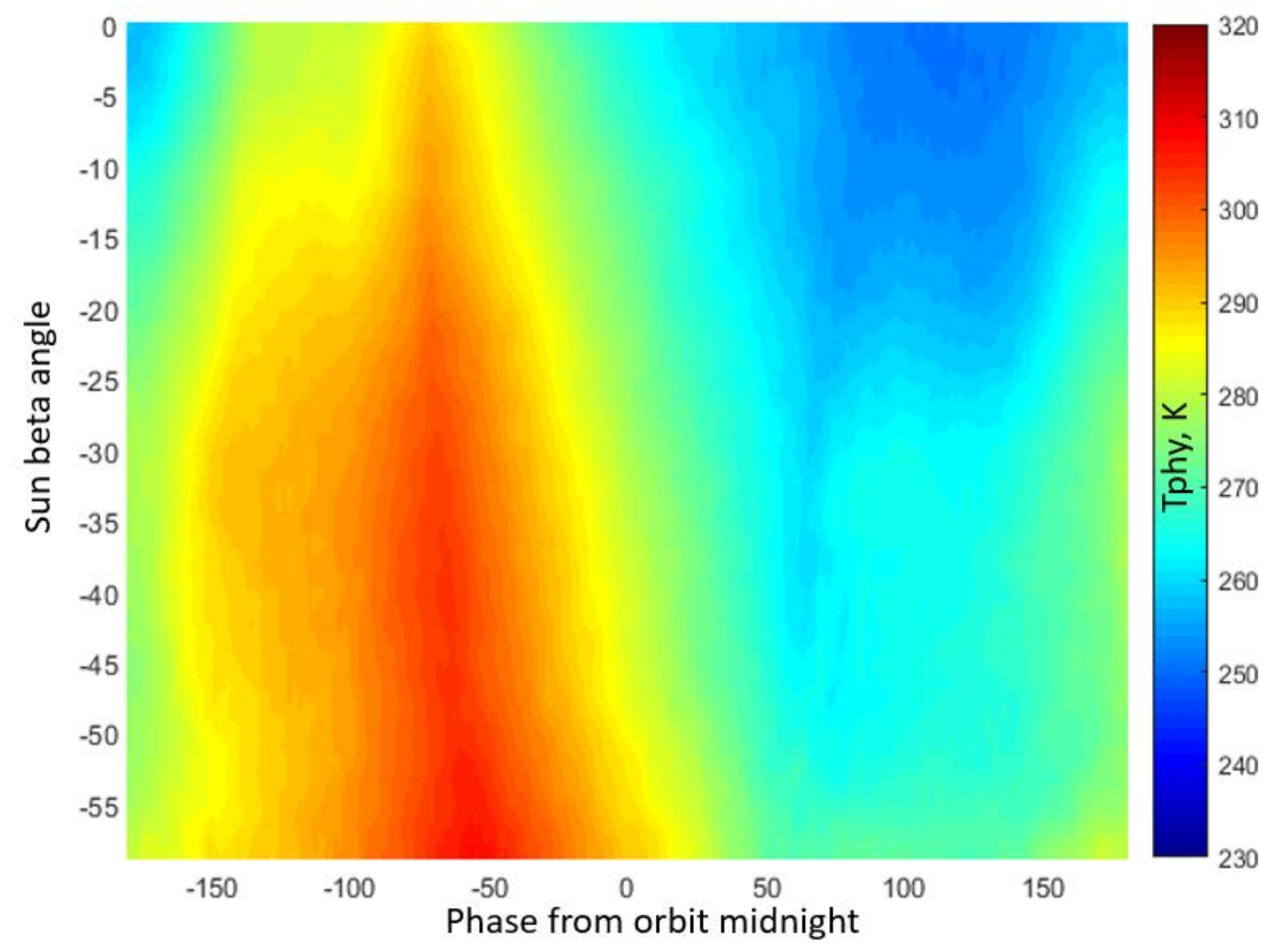

Figure 6.23: 2D matrix of MR physical temperature (yaw 180 and post-boost). 
APPENDIX F: DOUBLE DIFFERENCE (DD) RESULTS FOR VERSIONS 7 AND 8 


\section{$\underline{\text { F.1 DD Distribution }}$}
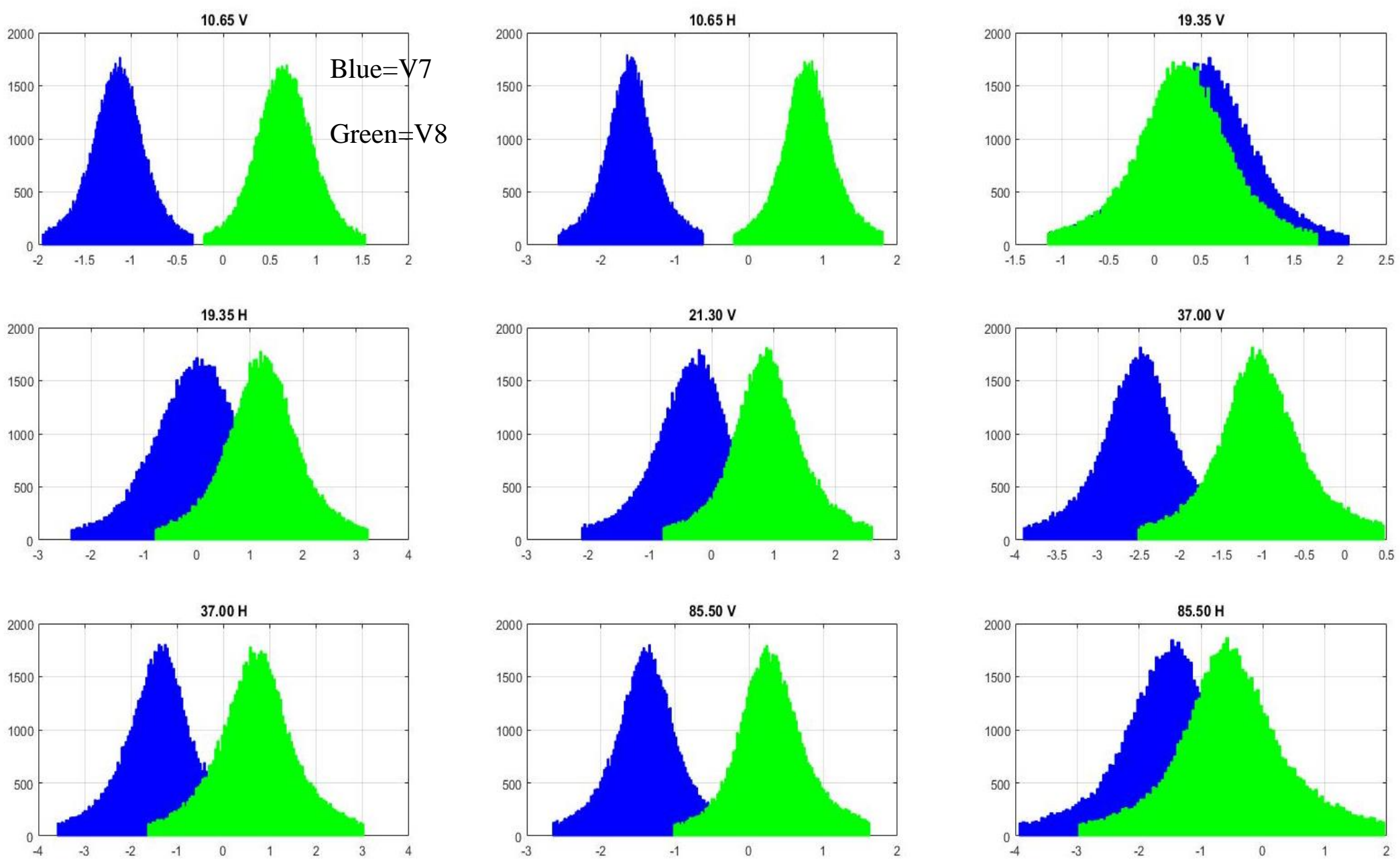

Figure 6.24: Double Difference (DD) distribution for V7 and V8 (all channels, yaw 0). 

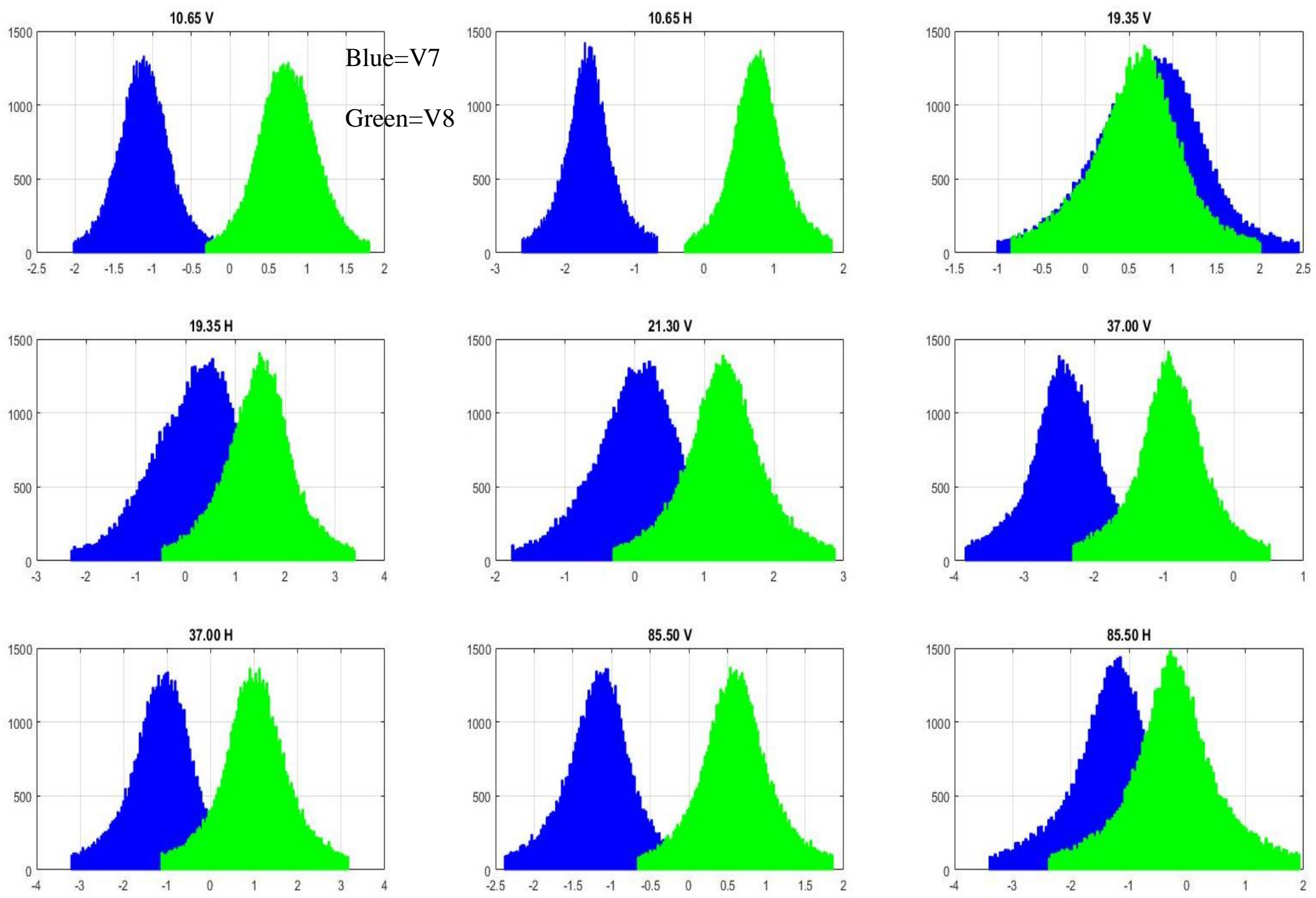

Figure 6.25: Double Difference (DD) distribution for V7 and V8 (all channels, yaw 180). 


\section{F.2 DD Based on Solar Coordinates}
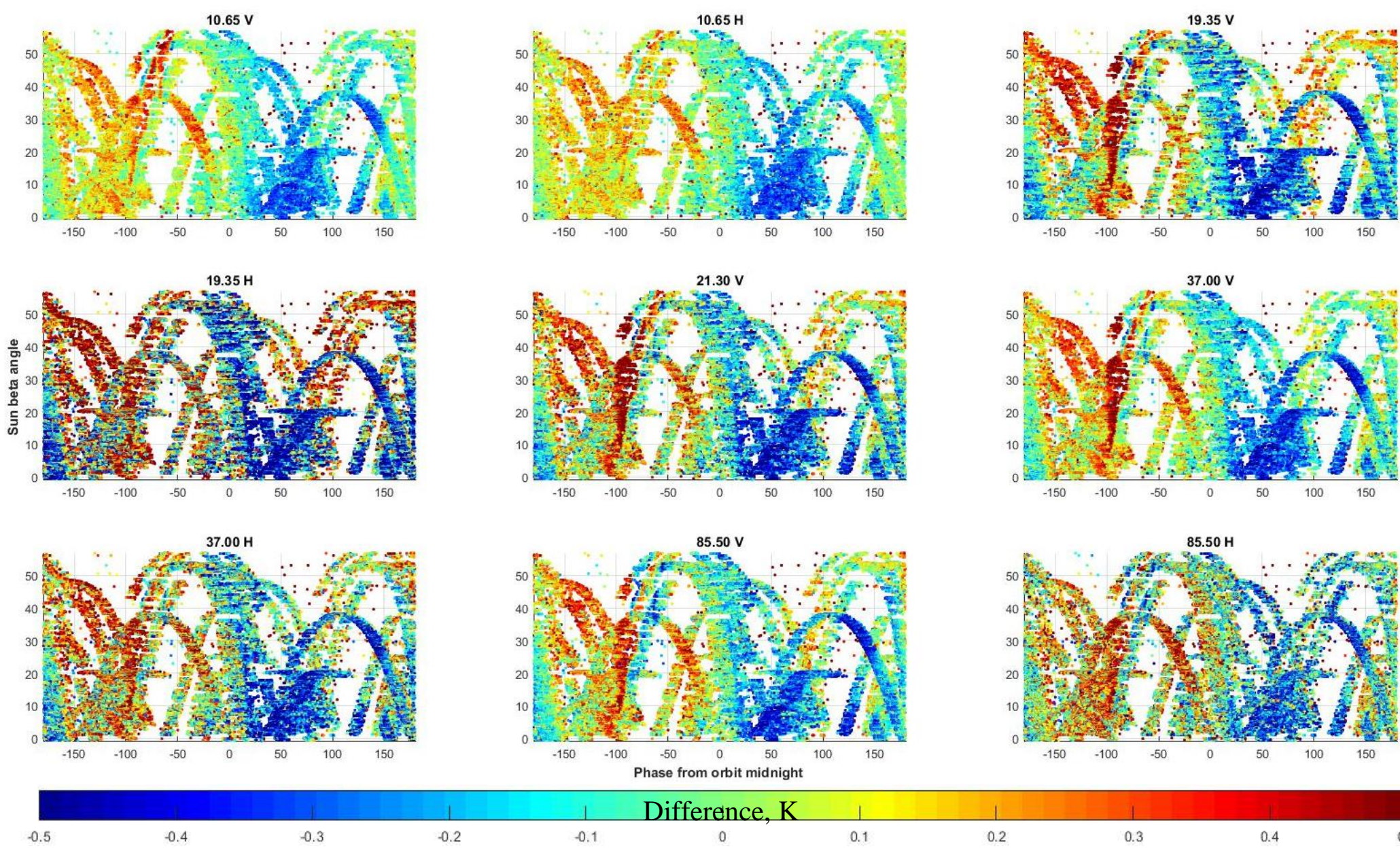

$-0.2$

Difference, $\mathrm{K}$

Figure 6.26: 2D Double Difference (DD) differences between V7 and V8 based on solar coordinates (all channels, yaw0). 

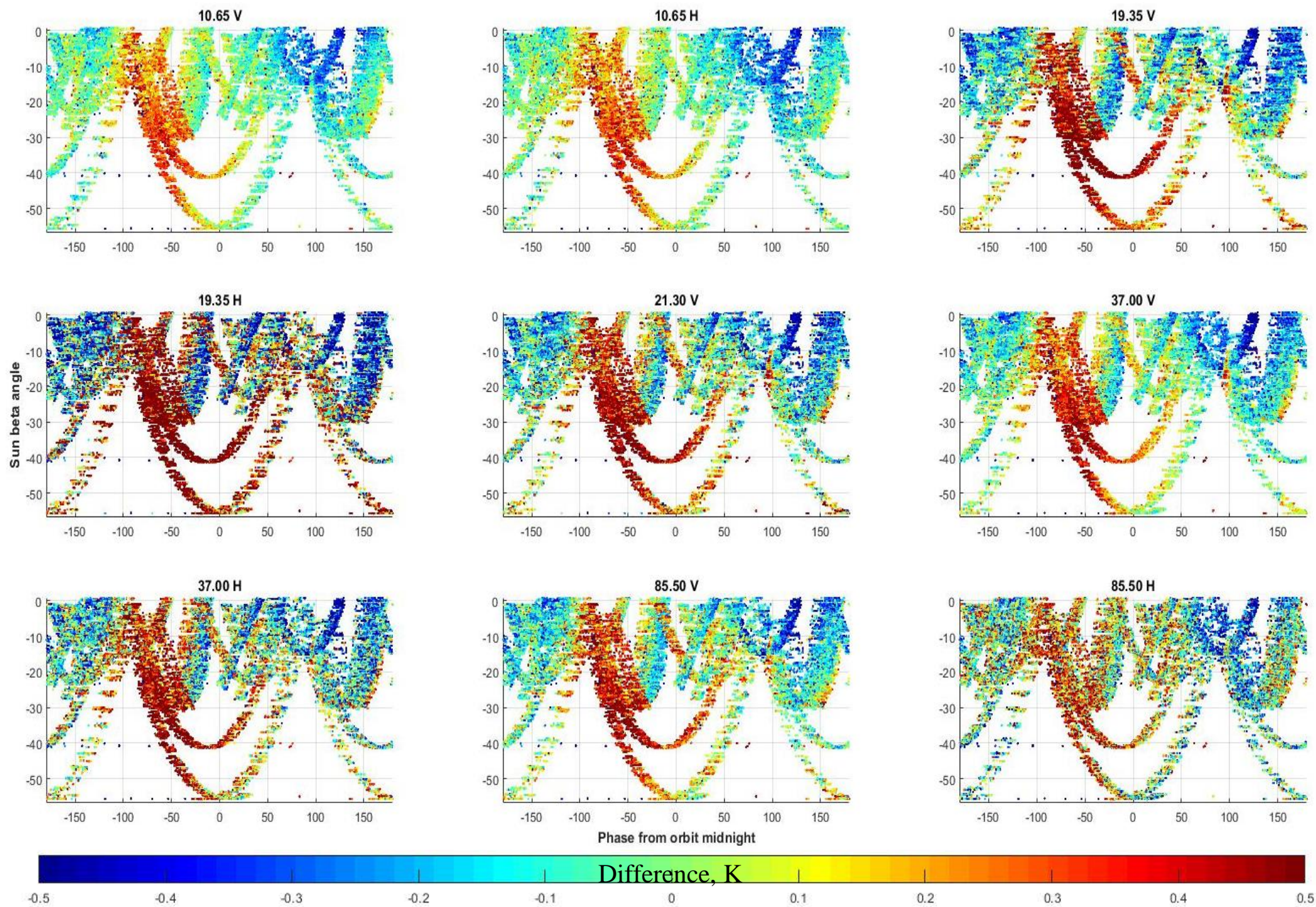

$-0.2$

Difference, $\mathrm{K}$

0.4

Figure 6.27: 2D Double Difference (DD) differences between V7 and V8 based on solar coordinates (all channels, yaw 180). 


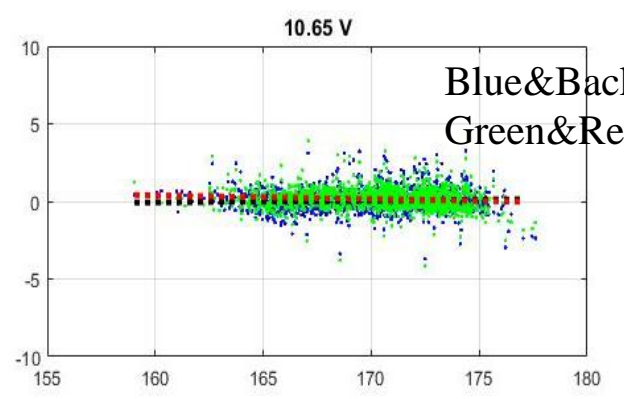

$10.65 \mathrm{H}$
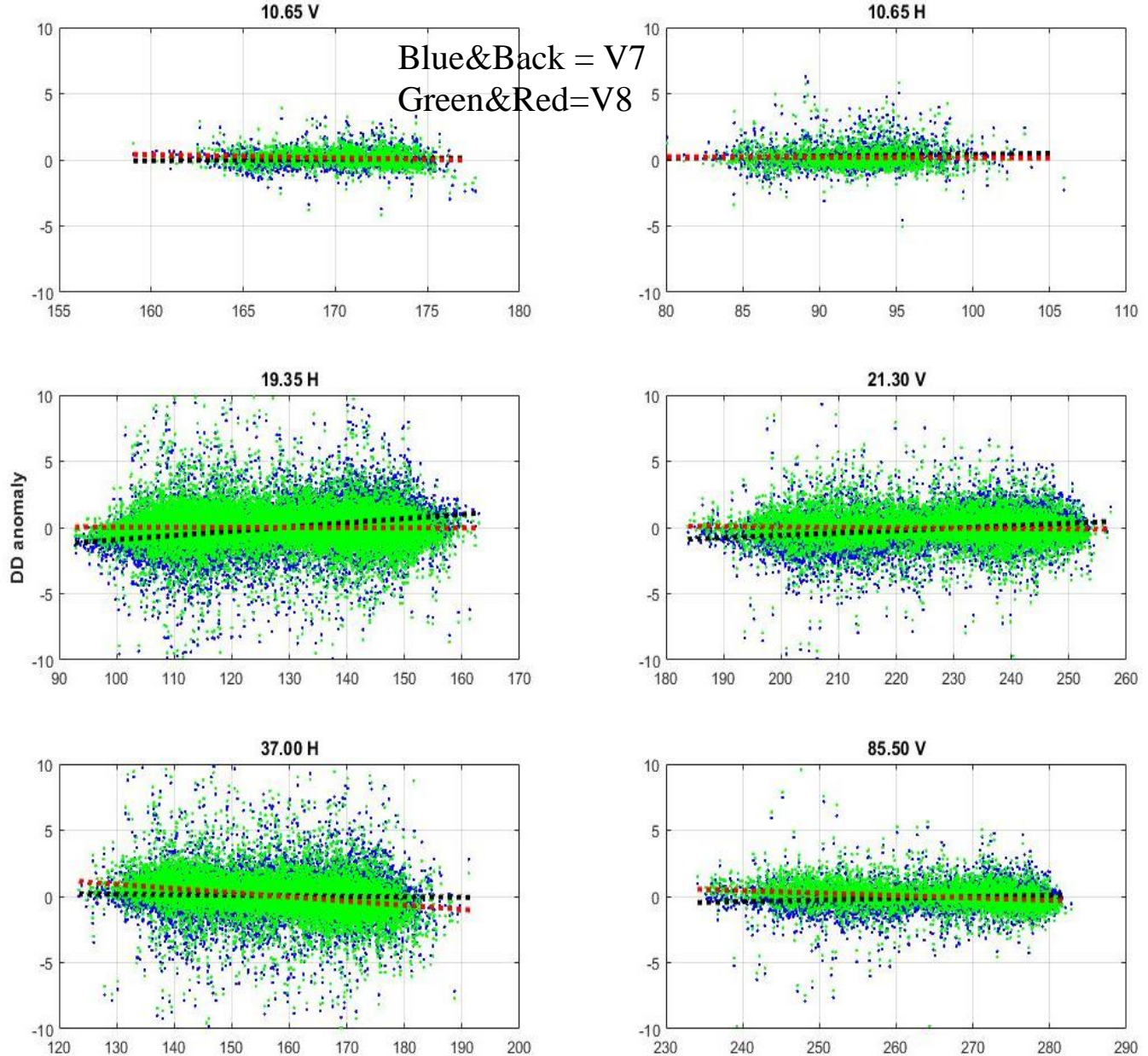

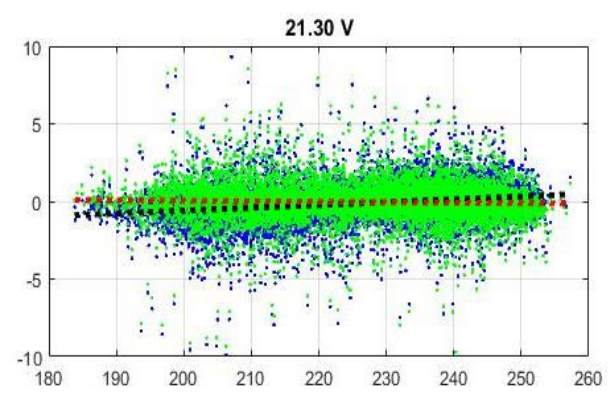

$85.50 \mathrm{~V}$

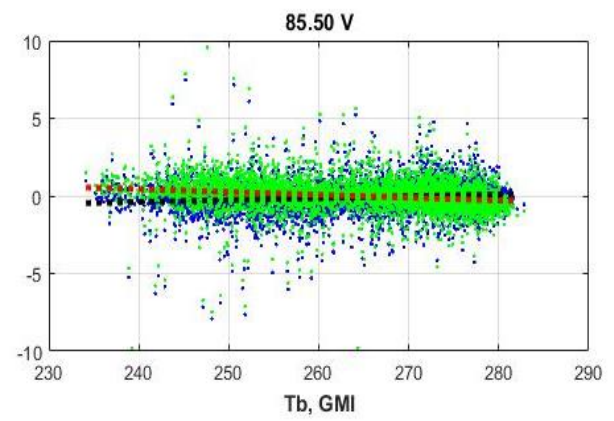

$19.35 \mathrm{~V}$
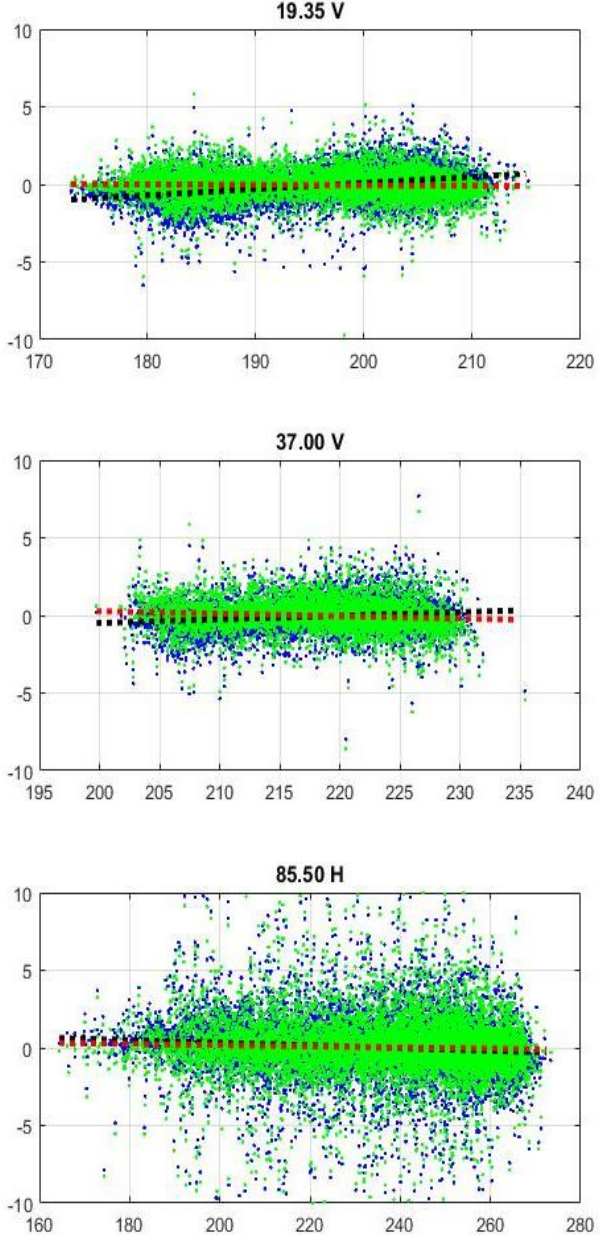

Figure 6.28: Scatter plot with linear regression for the high DD anomaly for V7 and V8 (all channels, yaw0) 

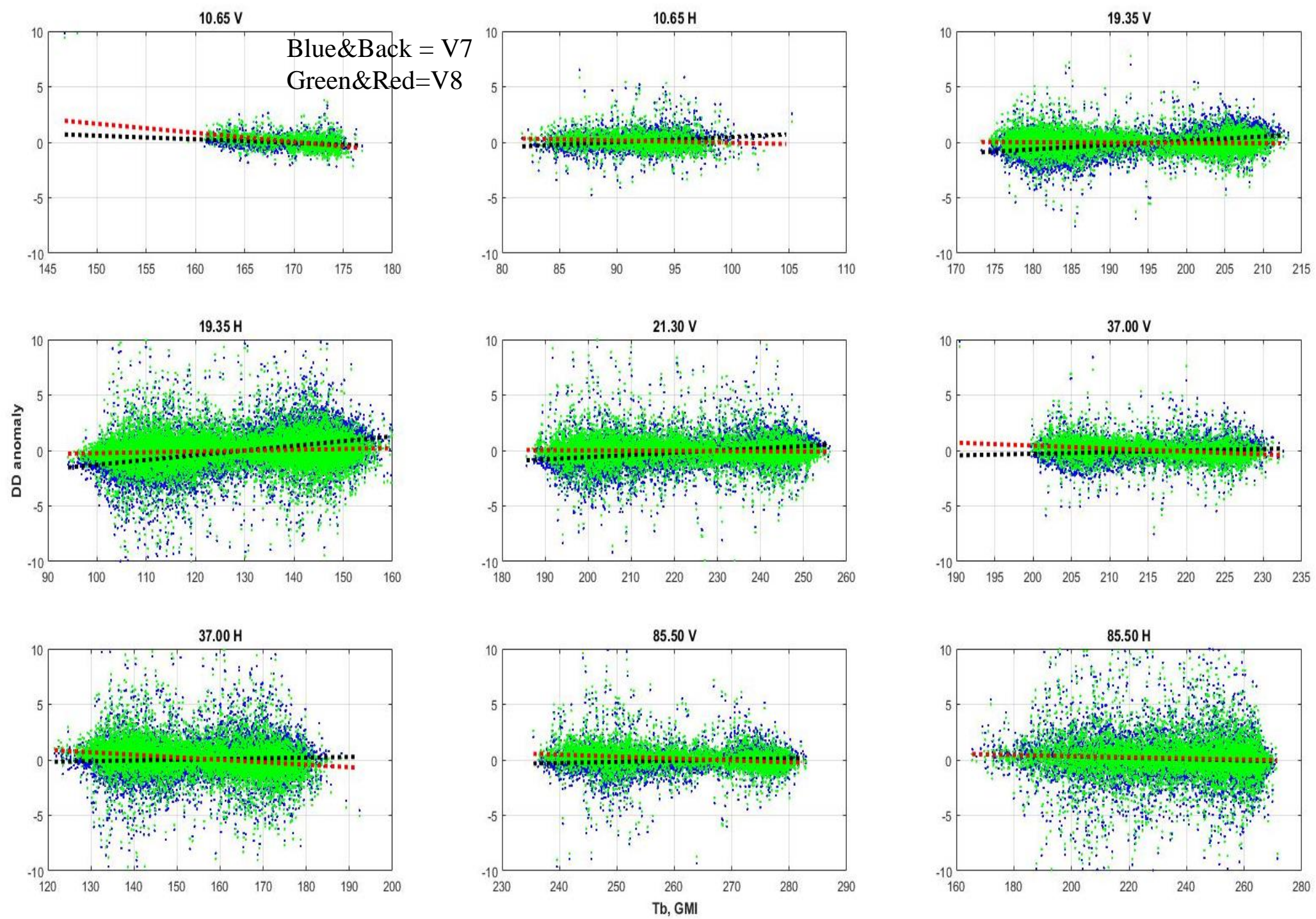

Figure 6.29: Scatter plot with linear regression for the high DD anomaly for V7 and V8 (all channels, yaw180) 